MATTHIAS MEIER

DAS SPARVERHALTEN DER PRIVATEN

HAUSHALTE UND DER

DEMOGRAPHISCHE

WANDEL:

MAKROÖKONOMISCHE

AUSWIRKUNGEN 


\section{DAS SPARVERHALTEN DER PRIVATEN HAUSHALTE UND DER DEMOGRAPHISCHE WANDEL: MAKROÖKONOMISCHE AUSWIRKUNGEN}

Wie könnte die Alterssicherung im Jahr 2030 aussehen? Diese Arbeit verbindet die privaten Haushalte, den Produktionssektor, die Rentenversicherung und die Bevölkerungsentwicklung in einem allgemeinen Gleichgewichtsmodell und analysiert die makroökonomischen Auswirkungen des demographischen Wandels. Ein besonderer Schwerpunkt liegt auf der Modellierung des Sparverhaltens der privaten Haushalte. Politikszenarien untersuchen, wie sich eine Anhebung des Rentenalters, eine höhere Zuwanderung, eine langsame Absenkung des Rentenniveaus und ein Umstieg zu einer kapitalgedeckten Alterssicherung auf wichtige makroökonomische Größen auswirken. Der Vergleich der Szenarien zeigt, daß die Beibehaltung der gegenwärtigen Regelungen das schlechteste Politikszenario ist. Also lohnt es sich, über Alternativen zur heutigen Rentenversicherung nachzudenken.

Matthias Meier studierte von 1986 bis 1991 Mathematik mit dem Nebenfach Volkswirtschaft an der Technischen Hochschule Darmstadt und an der Universität Mannheim. Von 1992 bis 1996 arbeitete er an der Universität Mannheim am Lehrstuhl für Makroökonomik und Wirtschaftspolitik. 1993/94 weilte der Autor für ein Jahr am University College London, um den Master of Science in Economics zu erwerben. 
Das Sparverhalten der privaten Haushalte und der demographische Wandel:

Makroökonomische Auswirkungen 


\section{ALLOKATION IM \\ MARKTWIRTSCHAFTLICHEN SYSTEM}

Herausgegeben von

Heinz König, Hans-Heinrich Nachtkamp,

Ulrich Schlieper, Eberhard Wille

Band 39

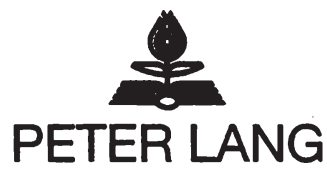

Frankfurt am Main - Berlin · Bern - New York - Paris - Wien

Matthias Meier - 978-3-631-75559-4

Downloaded from PubFactory at 01/11/2019 03:34:56AM

via free access 


\section{MATTHIAS MEIER}

\section{DAS SPARVERHALTEN DER PRIVATEN HAUSHALTE UND DER DEMOGRAPHISCHE WANDEL: MAKROÖKONOMISCHE AUSWIRKUNGEN}

Eine Simulation verschiedener Reformen der Rentenversicherung

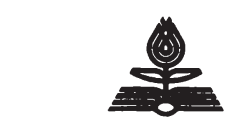

PETER LANG

Europäischer Verlag der Wissenschaften 
Die Deutsche Bibliothek - CIP-Einheitsaufnahme

Meier, Matthias:

Das Sparverhalten der privaten Haushalte und der demographische Wandel : makroökonomische Auswirkungen ; eine Simulation verschiedener Reformen der Rentenversicherung / Matthias Meier. - Frankfurt am Main ; Berlin ; Bern ; New York ; Paris ; Wien : Lang, 1997

(Allokation im marktwirtschaftlichen System ; Bd. 39)

Zugl.: Mannheim, Univ., Diss., 1997

ISBN 3-631-31762-X

Open Access: The online version of this publication is published on www.peterlang.com and www.econstor.eu under the international Creative Commons License CC-BY 4.0. Learn more on how you can use and share this work: http://creativecommons.org/licenses/ by/4.0.

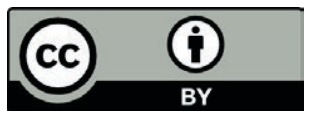

This book is available Open Access thanks to the kind support of ZBW - Leibniz-Informationszentrum Wirtschaft.

\author{
D 180 \\ ISSN 0939-7728 \\ ISBN 3-631-31762-X \\ ISBN 978-3-631-75559-4 (eBook) \\ (c) Peter Lang $\mathrm{GmbH}$ \\ Europäischer Verlag der Wissenschaften \\ Frankfurt am Main 1997 \\ Alle Rechte vorbehalten.
}

Das Werk einschließlich aller seiner Teile ist urheberrechtlich geschützt. Jede Verwertung außerhalb der engen Grenzen des Urheberrechtsgesetzes ist ohne Zustimmung des Verlages unzulässig und strafbar. Das gilt insbesondere für Vervielfältigungen, Übersetzungen, Mikroverfilmungen und die Einspeicherung und Verarbeitung in elektronischen Systemen.

Printed in Germany 123457 


\section{Danksagung}

Dieses Buch ist die überarbeitete Fassung meiner Doktorarbeit, die im Januar 1997 von der Fakultät für Volkswirtschaftslehre der Universität Mannheim angenommen wurde. Ich danke vor allem meinem akademischen Lehrer, Prof. Axel Börsch-Supan Ph.D., dessen konstruktive und kritische Betreuung für mich sehr wertvoll war. Mein Dank gilt auch Prof. Dr. Ulrich Schlieper, der als Zweitgutachter und Mitherausgeber dieser Buchreihe besonders für die Veröffentlichung hilfreiche Hinweise gegeben hat.

Als ehemaliger Assistent am Lehrstuhl Börsch-Supan möchte ich natürlich auch meinen Kolleginnen und Kollegen danken, die in vielen großen und kleinen Diskussionen zum Gelingen meiner Arbeit beigetragen haben: Angelika Eymann, Markus Homann, Anette Reil-Held, Ralf Rodepeter, Peter Schmidt und Reinhold Schnabel. Wichtig ist mir auch, den studentischen Mitarbeiterinnen und Mitarbeitern des Lehrstuhls zu danken, die sowohl fachlich als auch menschlich manche Unterstützung geleistet haben. Letzteres gilt ganz besonders für die „Managerin“ des Lehrstuhls: Isabella Nohe.

$\mathrm{Da} ß$ eine Dissertation einen hohen zeitlichen Einsatz bedeutet, ist selbstverständlich. Nicht selbstverständlich war das Einfühlungsvermögen mir nahestehender Menschen, ohne die meine Arbeit nicht entstanden wäre. Deshalb schließe ich mit einem ganz besonders herzlichen Dank an Katharina Spieß, Mechthild, Gerhard und Brigitta Meier sowie Elisabeth Schlott und Else Meier. 
Matthias Meier - 978-3-631-75559-4

Downloaded from PubFactory at 01/11/2019 03:34:56AM

via free access 


\section{Inhaltsverzeichnis}

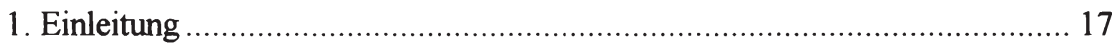

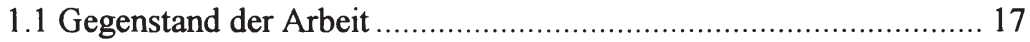

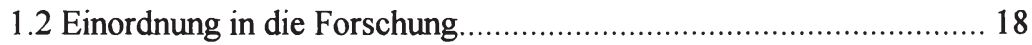

1.3 Aufbau der Arbeit ..................................................................... 24

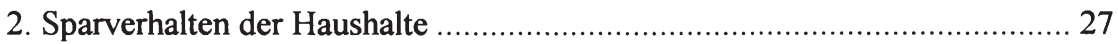

2.1 Klassisches Lebenszyklusmodell............................................ 28

2.1.1 Theoretische Betrachtung ............................................. 28

2.1.2 Empirische Evidenz - Ergebnisse aus der Literatur ............. 31

2.2 Erweiterungen des klassischen Lebenszyklusmodells .................... 36

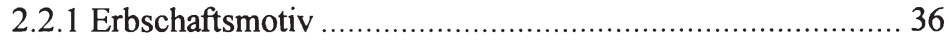

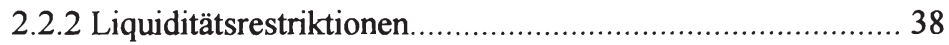

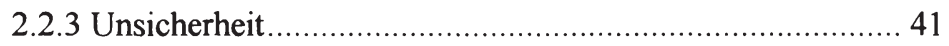

2.2.4 Andere Erweiterungen................................................... 44

2.2.5 Kritische Würdigung ...................................................... 45

2.3 Mental Accounting und Sustainable Consumption........................ 46

2.3.1 Mental Accounting......................................................... 46

2.3.2 Sustainable Consumption ............................................... 51

2.3.3 Simulation des Mental Accounting Modells ..................... 58

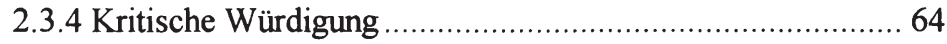

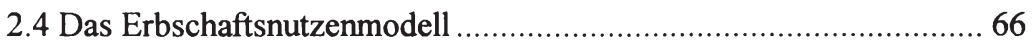

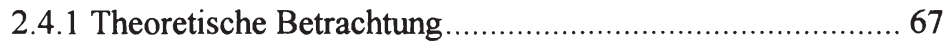

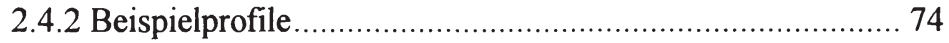

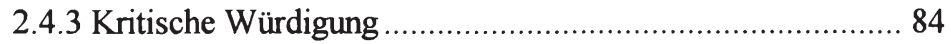




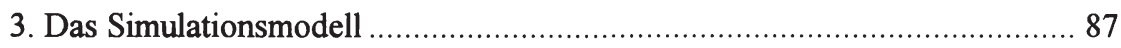

3.1 Der Haushaltssektor............................................................ 87

3.1.1 Das Erbschaftsnutzenmodell als Haushaltsmodell.............. 87

3.1.2 Endogenes Arbeitsangebot ........................................... 88

3.1.3 Spezifikation der Lebensnutzenfunktion .......................... 91

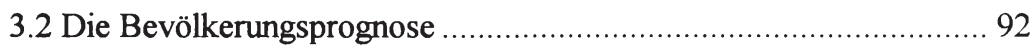

3.2.1 Das Prognoseverfahren ................................................ 92

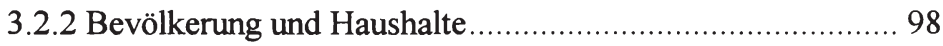

3.2.3 Tod: Vererben und Erben ................................................ 101

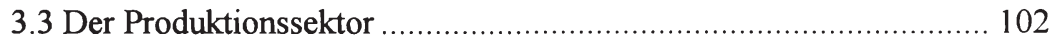

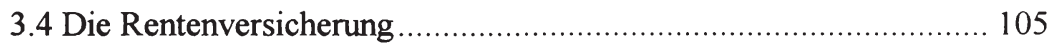

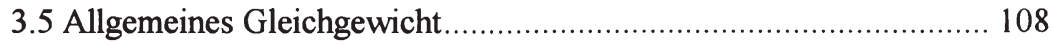

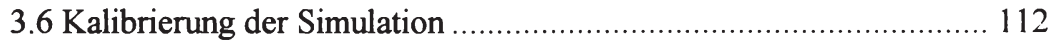

3.6.1 Auswahl der zu kalibrierenden Variablen ........................ 113

3.6.2 Methode und Ergebnisse der Kalibrierung ...................... 121

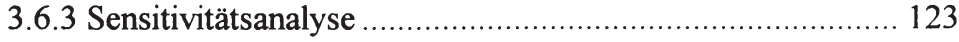

3.6.4 Konvergenz zum Steady-State .................................... 125

4. Ergebnisse des Simulationsmodells ................................................. 129

4.1 Das Basisszenario ................................................................... 130

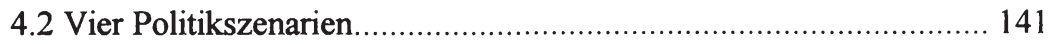

4.3 Kleine offene Volkswirtschaft................................................... 155

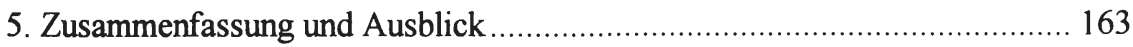

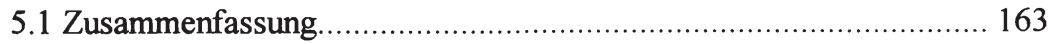

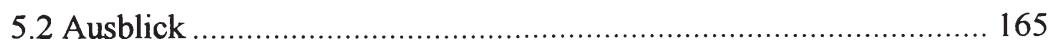




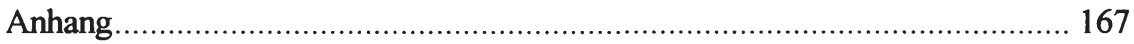

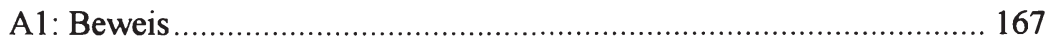

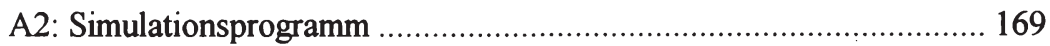

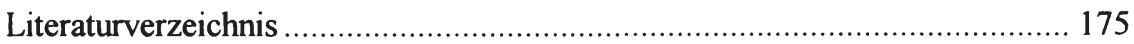


Matthias Meier - 978-3-631-75559-4

Downloaded from PubFactory at 01/11/2019 03:34:56AM

via free access 


\section{Tabellenverzeichnis}

Tabelle 2.1: $\quad$ Abweichung des Sustainable Consumption Optimums vom Optimum des klassischen Lebenszyklusmodells 54

Tabelle 3.1: Durchschnittliche Lebenserwartung Neugeborener in Jahren 93

Tabelle 3.2: Zusammensetzung, durchschnittliche Größe und Anzahl der Haushalte einer Kohorte k........................................ 99

Tabelle 3.3: Erbschaftsbeziehungen für eine Kohorte k.......................... 101

Tabelle 3.4: Sensitivität von endogenen Variablen im Jahr 2040 .............. 124

Tabelle 4.1: Das Basisszenario und die vier Politikszenarien ................... 144

Tabelle 4.2: Anteil der Erbschaften am Lebensarbeitseinkommen in Prozent 159 
Matthias Meier - 978-3-631-75559-4

Downloaded from PubFactory at 01/11/2019 03:34:56AM

via free access 


\section{Abbildungsverzeichnis}

Abbildung 2.1: Konsum-, Arbeitseinkommens- und Vermögensprofil im klassischen Lebenszyklusmodell ....................................... 30

Abbildung 2.2: Konsumnutzen im Mental Accounting Modell.................... 48

Abbildung 2.3: Sustainable Consumption im Vergleich zum klassischen Lebenszyklusmodell........................................................ 56

Abbildung 2.4: Mental Accounting Konsumprofile..................................... 61

Abbildung 2.5: Mental Accounting Vermögensprofile ............................... 63

Abbildung 2.6: Marginaler Erbschaftsnutzen.............................................. 76

Abbildung 2.7: Konsumprofile im Erbschaftsnutzenmodell und im Lebenszyklusmodell bei unsicherer Lebenserwartung ohne Erbschaftsmotiv..................................................... 77

Abbildung 2.8: MRSA im Erbschaftsnutzenmodell ................................... 79

Abbildung 2.9: Konsumprofile im Erbschaftsnutzenmodell bei unterschiedlichen Arbeitseinkommensprofilen..................... 80

Abbildung 2.10: Vermögensprofile im Erbschaftsnutzenmodell bei unterschiedlichen Arbeitseinkommensprofilen...................... 81

Abbildung 2.11: Konsumprofile im Erbschaftsnutzenmodell bei unterschiedlicher Arbeitseinkommenshöhe.

Abbildung 2.12: Vermögensprofile im Erbschaftsnutzenmodell bei unterschiedlicher Arbeitseinkommenshöhe.

Abbildung 3.1: Alterszusammensetzung der Einwanderer und der Einheimischen im Jahr 1989 95

Abbildung 3.2: Bevölkerungsstruktur in Westdeutschland in den Jahren 1989 und 2040 96

Abbildung 3.3: Anteil der Kinder, Erwerbspersonen und Personen im Rentenalter an der Gesamtbevölkerung in Westdeutschland zwischen 1996 und 2050 97

Abbildung 3.4: Das Simulationsmodell und seine Teilmodelle 110

Abbildung 3.5: Schema des Simulationsprogramms. 
Abbildung 3.6: Entwicklung der Kapitalelastizität zwischen 1970 und 1989

Abbildung 3.7: Entwicklung der Abschreibungsrate zwischen 1970 und 1989

Abbildung 3.8: Sparquote nach dem Alter des Haushaltsvorstands in den EVS 1973, 1978, 1983 und 1988

Abbildung 3.9: Vermögensquote nach dem Alter des Haushaltsvorstands in den EVS 1973, 1978, 1983 und 1988

Abbildung 3.10: Erwerbsquote nach dem Alter für 1974, 1979, 1984 und 1989

Abbildung 3.11: Entwicklung des Netto-Kapitalkoeffizienten zwischen 1970 und 1989

Abbildung 3.12: Prozentuale Veränderung der Kapitalintensität 126

Abbildung 4.1: Entwicklung der Kapitalintensität (Index $1996=1$ )

Abbildung 4.2: Entwicklung der Sparquote und des Zinssatzes

Abbildung 4.3: Durchschnittlich erhaltene Erbschaften im Basisszenario.

Abbildung 4.4: Entwicklung des Beitragssatzes zur umlagefinanzierten

Rentenversicherung.

Abbildung 4.5: Entwicklung der Erwerbsstundenquote. 137

Abbildung 4.6: Entwicklung des Pro-Kopf-Konsums (Index 1996=1)

Abbildung 4.7: Entwicklung der Kapitalintensität in den Politikszenarien (Index 1996 = 1)

Abbildung 4.8: Entwicklung der Sparquote in den Politikszenarien 146

Abbildung 4.9: Entwicklung des Beitragssatzes zur umlagefinanzierten

Rentenversicherung in den Politikszenarien. 148

Abbildung 4.10: Summe aus Sparquote und Beitragssatz in den

Politikszenarien

Abbildung 4.11: Entwicklung der Erwerbsstundenquote in den

Politikszenarien.

Abbildung 4.12: Entwicklung des Pro-Kopf-Konsums in den Politikszenarien (Index $1996=1$ ) 
Abbildung 4.13: Entwicklung des Kapitalbesitzes im Ausland in Prozent des inländischen Outputs.

Abbildung 4.14: Entwicklung der Erwerbsstundenquote in der kleinen offenen Volkswirtschaft

Abbildung 4.15: Entwicklung des Beitragssatzes zur umlagefinanzierten

Rentenversicherung in der kleinen offenen

Volkswirtschaft.

Abbildung 4.16: Entwicklung des Pro-Kopf-Konsums in der kleinen offenen Volkswirtschaft (Index $1996=1$ ) 
Matthias Meier - 978-3-631-75559-4

Downloaded from PubFactory at 01/11/2019 03:34:56AM

via free access 


\section{Einleitung}

\subsection{Gegenstand der Arbeit}

Bis vor kurzem war von Politikern immer wieder das Schlagwort „Die Renten sind sicher!" zu hören. Mittlerweile ist man durch die aktuellen Finanzprobleme der Rentenversicherung mit derartigen Äußerungen etwas vorsichtiger geworden, doch noch immer wird argumentiert, daß die Renten langfristig sicher wären und die aktuelle Finanzmisere auf die aktuellen Probleme - die konjunkturelle Lage und die hohe Arbeitslosigkeit - zurückzuführen sei. Der zweite Teil der Behauptung - die aktuelle Finanzmisere wird durch aktuelle Probleme verursacht - ist zweifellos richtig, aber wenn man sich vor Augen führt, daß der demographische Wandel gerade in Deutschland zu einem erheblich höheren Anteil von älteren Menschen führen wird, sind Zweifel an der optimistischen langfristigen Sichtweise der Politik angebracht.

Tatsächlich ist schon heute klar vorauszusehen, daß sich das Verhältnis der Bevölkerung im Rentenalter zur Bevölkerung im Erwerbsalter bis zum Jahr 2030 in etwa verdoppeln wird. Der Begriff „Alterspilz“ in Abgrenzung zur Alterspyramide bringt das daraus erwachsende Problem auf den Punkt: Vielen Rentnern (sie sind der Kopf des Pilzes) stehen relativ wenige Erwerbstätige (sie sind der Stamm des Pilzes) gegenüber. Aus dieser Verschiebung der Altersstruktur können sich gravierende Probleme für die sozialen Sicherungssysteme ergeben. Eine überschlägige Berechnung der zum Ausgleich der Rentenfinanzen nötigen Beitragssätze ergibt im jetzigen System Höchstsätze von ca. $30 \%$ um das Jahr 2030. Da auch die Ausgaben der Kranken- und Pflegeversicherung sehr stark von der Altersstruktur der Bevölkerung abhängen, ist eine Belastung des Bruttolohnes allein mit Sozialversicherungsbeiträgen von nahezu 50 \% im Jahr 2030 durchaus möglich. Es ist kaum zu bestreiten, daß sich bei dieser Gesamtbelastung enorme Verzerrungen im Arbeitsangebot mit starken Tendenzen in Richtung Schattenwirtschaft ergeben werden.

Ob im Jahr 2030 tatsächlich Sozialversicherungsbeiträge von $50 \%$ auf die Arbeitnehmer zukommen hängt davon $a b$, wie die Rentenversicherung in den nächsten Jahrzehnten weiterentwickelt wird. Neben der Heraufsetzung des Rentenalters und einer höheren Einwanderung im System der bisherigen umlagefinanzierten Rentenversicherung bietet sich auch der teilweise oder vollständige Umstieg auf eine kapitalgedeckte Alterssicherung an. Welche makroökonomischen Auswirkungen sich aus diesen möglichen Weiterentwicklungen ergeben, ist die zentrale Frage meiner Arbeit.

Diese Frage analysiere ich in einem intertemporalen allgemeinen Gleichgewichtsmodell, denn die Rentenversicherung ist wie der Produktions- und Haushaltssek- 
tor endogener Bestandteil der Gesamtwirtschaft. Während ich den Produktionssektor mit einem relativ einfachen Modell beschreibe, widme ich dem Haushaltssektor breiten Raum. Zwei Aspekte des demographischen Wandels sind dafür ausschlaggebend: Erstens können die Haushalte ihren Konsum nach der Verrentung nicht nur mit ihrem Renteneinkommen, sondern auch mit Ersparnissen finanzieren, und zweitens induziert eine Veränderung des Altersaufbaus der Bevölkerung bei altersabhängigen Sparprofilen Veränderungen des gesamtwirtschaftlichen Kapitalangebots.

Bestehende Arbeiten zum Thema „Makroökonomische Auswirkungen des demographischen Wandels" modellieren das Konsum- und Sparverhalten der Haushalte mit einfachen Versionen des Lebenszyklusmodells. Die einfache - und deswegen restriktive - Struktur des Lebenszyklusmodells ist ein Problem. Zahlreiche empirische Arbeiten belegen, daß sich das beobachtete Sparverhalten der Haushalte nur wenig mit den Aussagen des Lebenszyklusmodells deckt. Ein Schwerpunkt meiner Arbeit liegt deshalb auf einer flexibleren Beschreibung des Sparverhaltens der Haushalte. Darauf aufbauend konstruiere ich ein allgemeines Gleichgewichtsmodell, das die Teilmodelle Haushaltssektor, Produktionssektor, Rentenversicherung und Bevölkerungsprognose verbindet. Dieses Modell kalibriere ${ }^{1}$ ich für Westdeutschland vor der Wiedervereinigung mit Daten aus der volkswirtschaftlichen Gesamtrechnung sowie kohortenspezifischen Daten aus mehreren Wellen der Einkommens- und Verbrauchsstichprobe. Dann wende ich das Modell an und simuliere verschiedene Szenarien, die weiterentwickelte Rentenversicherungen studieren. Mein Ziel ist es, mit den Ergebnissen der Simulationen die zentrale Frage meiner Arbeit zu untersuchen: Welche makroökonomischen Auswirkungen hat eine weiterentwickelte Rentenversicherung angesichts des demographischen Wandels?

\subsection{Einordnung in die Forschung}

In diesem kurzen Literaturüberblick bringe ich die wichtigsten Simulationsstudien zu den makroökonomischen Auswirkungen des demographischen Wandels. Auf diesen Studien baut meine Arbeit auf. Die entscheidende Neuerung meiner Arbeit ist die Berücksichtigung der unsicheren Lebenserwartung, durch die Accidental Bequests möglich werden. In einem allgemeinen Gleichgewichtsmodell ist dieser

1 Kalibrieren bedeutet, ausgewählte endogene Variablen des Modells an tatsächliche Daten anzupassen, damit das Modell die Realität in einem bestimmten Zeitraum in den ausgewählten Dimensionen möglichst gut beschreibt. 
Aspekt des demographischen Wandels meines Wissens bisher nicht simuliert worden.

Aus theoretischer Sicht hat Abel (1985) die Rolle von Accidental Bequests in einem allgemeinen Gleichgewichtsmodell untersucht. In seinem Modell haben die Konsumenten zwei Lebensabschnitte, wobei der zweite Lebensabschnitt nur mit einer gewissen Wahrscheinlichkeit erreicht wird. Abel zeigt, daß eine versicherungsmathematisch faire Sozialversicherung die gesamtwirtschaftliche Kapitalbildung reduziert, sofern kein Markt für Annuitäten besteht. Dies ist ein wichtiges Argument für eine kapitalgedeckte Alterssicherung, denn wenn die private Ersparnis steigt, dann stehen mehr Mittel für Investitionen zur Verfügung. Auf den Zusammenhang zwischen Ersparnis (Konsumverzicht) und Investitionen weist die Deutsche Bundesbank immer wieder deutlich hin:

„Auf längere Sicht setzt eine Zunahme der Produktion und damit auch der zukünftigen Konsummöglichkeiten die Schaffung neuer und zusätzlicher Kapazitäten voraus. Die hierzu notwendigen Investitionen sind letztlich nur durch einen entsprechenden Konsumverzicht möglich." (Deutsche Bundesbank, 1996, S. 17)

Die Ergebnisse Abels sind wegen der wechselseitigen Abhängigkeiten im allgemeinen Gleichgewicht rein qualitativ. Um auch quantitative Ergebnisse zu erhalten, simuliere ich in dieser Arbeit das allgemeine Gleichgewicht bei unsicherer Lebenserwartung und berücksichtige die dadurch entstehenden Accidental Bequests.

Auerbach und Kotlikoff (1987)

Ein Meilenstein in der Entwicklung makroökonomischer Simulationen ist die Monographie Dynamic Fiscal Policy, in der Auerbach und Kotlikoff ein allgemeines Gleichgewichtsmodell unter der Annahme perfekter Voraussicht simulieren. Ich fasse die wesentlichen Gedanken dieses Modells kurz zusammen, weil sie der Ausgangspunkt meiner Arbeit sind.

Das Grundmodell von Auerbach und Kotlikoff beschreibt eine geschlossene Volkswirtschaft mit drei Sektoren: den Haushalten, den Firmen und dem Staat. Die Haushalte (genauer: ein repräsentativer Haushalt je Kohorte) wählen für gegebene Löhne, Steuersätze und Zinsen ihre Konsumnachfrage und Arbeitsangebot mit dem Ziel, ihren Lebensnutzen zu maximieren. Der Produktionssektor wird in einem stark stilisierenden Modell abgebildet, in dem eine repräsentative Firma bei gegebenen Löhnen und Zinsen über den optimalen Einsatz der Produktionsfaktoren Arbeit und Kapital entscheidet. Der Staat erhebt Steuern, um seine Ausgaben zu finanzieren und betreibt ein Sozialversicherungssystem, das über lohnbezo- 
gene Pflichtbeiträge finanziert wird. Das allgemeine Gleichgewichtsmodell schließt das System, indem es die Löhne, Zins- und Steuersätze für jedes Jahr so bestimmt, daß Angebot und Nachfrage nach Kapital und Arbeit übereinstimmen und die Budgetrestriktionen des Staates und der Sozialversicherung erfüllt sind. Zusätzlich fordert die Annahme perfekter Voraussicht, daß die Pläne der beteiligten Akteure mit den Gleichgewichtswerten übereinstimmen müssen. Aus der Simulation des Modells kann man lernen, wie sich die endogenen Variablen in dem interessierenden Zeitraum entwickeln.

Der entscheidende Fortschritt von Auerbach und Kotlikoff ist die endogene Sichtweise, denn Steuersätze und Beitragssätze zur Sozialversicherung verzerren Entscheidungen: Die Akteure substituieren hoch belastete Einkommensarten zugunsten von weniger belasteten Einkommensarten. Diese Substitutionseffekte und wechselseitigen Abhängigkeiten gehen in Teilmodellen, die beispielsweise die Budgetrestriktion der Rentenversicherung mit der Bevölkerungsentwicklung kombinieren, verloren.

Auerbach, Kotlikoff, Hagemann, Nicoletti (1989)

Eine spezielle Anwendung ihres Simulationsmodells auf die Folgen des demographischen Wandels präsentieren Auerbach et al. in einer Studie, die Japan, Schweden, die USA und Deutschland vergleicht. Darin beschreiben sie das Konsumund Sparverhalten der Haushalte mit dem klassischen Lebenszyklusmodell nach Modigliani und Brumberg (1954) und erweitern dieses Modell um ein Erbschaftsmotiv bei sicherer Lebenserwartung sowie ein endogenisiertes Arbeitsangebot. Als viertes wichtiges Element neben den drei Sektoren der Volkswirtschaft vervollständigt eine Bevölkerungsprognose das Simulationsmodell. Um ihre Simulation gegebenen Daten aus den volkswirtschaftlichen Gesamtrechnungen der jeweiligen Länder anpassen zu können, müssen Auerbach et al. sowohl ein sehr hohes Gewicht des Erbschaftsmotivs als auch eine negative Zeitpräferenzrate annehmen. Für Deutschland verwenden sie beispielsweise eine Zeitpräferenzrate von $-5,5 \%$, also gewichten die Haushalte die Zukunft stärker als die Gegenwart - eine sehr gewagte Hypothese. Trotzdem sagen Auerbach et al. eine dramatisch sinkende aggregierte Sparquote voraus: Sie fällt in ihrem Basisszenario von 19,7 \% im Jahr 1990 auf 2,7 \% im Jahr 2050.

An der problematischen Kalibrierung wird deutlich, daß das klassische Lebenszyklusmodell an seine Grenzen stößt: Es ist zu restriktiv, um das beobachtete Haushaltsverhalten zu beschreiben. Meine Arbeit übernimmt die Grundstruktur des Simulationsmodells von Auerbach et al., entwickelt aber ein weniger restriktives Haushaltsmodell, um so eine Kalibrierung mit akzeptableren Parametern zu ermöglichen. 
Außer ihrem Basisszenario untersuchen Auerbach et al. verschiedene Politikszenarien, wobei neben verschiedenen fiskalischen Maßnahmen auch das Rentenalter variiert wird. Diese Szenarien gehen von einer geschlossenen Volkswirtschaft aus. Der geschlossenen Volkswirtschaft stellen Auerbach et al. in einem weiteren Szenario die Situation in einer kleinen offenen Volkswirtschaft gegenüber.

In einer neueren Anwendung des Modells untersucht Kotlikoff (1996), ob ein pareto-verbessernder Übergang vom Umlageverfahren zum Kapitaldeckungsverfahren konstruiert werden kann. Ein solcher Systemwechsel ist nach Aaron (1966) vorteilhaft, wenn die Summe der Wachstumsrate der Bevölkerung und der Wachstumsrate der Löhne kleiner ist als der Zins. Daher wird das Kapitaldeckungsverfahren bei einer negativen Wachstumsrate der Bevölkerung zunehmend attraktiv. Kotlikoff bestreitet nicht die langfristigen Vorteile des Kapitaldeckungsverfahrens, die Realisierungschancen für einen pareto-verbessernden Übergang schätzt er jedoch gering ein.

Cutler, Poterba, Sheiner, Summers (1990)

Die Simulation von Cutler et al. unterscheidet sich deutlich von Auerbach et al. (1989). Nachdem Cutler et al. den demographischen Wandel in den USA studiert haben, untersuchen sie, wie freier Kapitalverkehr die Situation verändert. Ihre Hypothese ist die folgende: Wenn verschiedene Länder eine unterschiedliche demographische Entwicklung durchmachen, dann könnte ein ungehinderter Kapitalverkehr die negativen Auswirkungen des Alterungsprozesses abmildern. Das in den alternden Ländern frei werdende Kapital könnte in Ländern mit wachsender junger Bevölkerung für beide Seiten nutzbringend angelegt werden. Diese wichtige Hypothese untersuchen Cutler et al. in einem Zwei-Länder-Modell, das die wechselseitigen Abhängigkeiten explizit berücksichtigt. In diesem Modell hat der Haushaltssektor eine einfachere Struktur als bei Auerbach et al., denn Cutler et al. gründen ihre Arbeit auf das Ramsey-Modell (siehe Barro and Sala-i-Martin, 1995), in dem die Haushalte (genauer: ein für den ganzen Sektor repräsentativer Haushalt mit unendlichem Planungshorizont) über ihren Konsumnutzen maximieren, die Freizeit (das Arbeitsangebot) aber als exogen ansehen. Dafür erweitern Cutler et al. das Produktionsmodell um technischen Fortschritt. Die Simulation zeigt, daß sowohl der technische Fortschritt als auch die offenen Kapitalmärkte die negativen Folgen des Alterungsprozesses abmildern oder sogar aufheben können. Wegen des exogenen Arbeitsangebotes versuchen Cutler et al. aber nicht, Reformen der Rentenversicherung zu diskutieren, denn veränderte Beitragssätze und Ersatzquoten können erhebliche Substitutionseffekte auslösen, die in ihrer Simulation nicht abgebildet werden können. 
Ein weiterer Schwerpunkt der Arbeit von Cutler et al. ist der Zusammenhang zwischen demographischen Veränderungen und technischem Fortschritt. Ihre These lautet: Wenn die Arbeitskräfte durch das Altern der Bevölkerung relativ knapp werden, dann könnte diese Knappheit einen schnelleren technischen Fortschritt verursachen. Mit dem Vergleich der Wachstumsraten der Erwerbstätigen und der Rate des technischen Fortschritts in verschiedenen Länder untermauern Cutler et al. ihre These.

Die Schlußfolgerungen von Cutler et al. sind daher weit weniger pessimistisch als die Schlußfolgerungen von Auerbach et al. (1989), die einen extremen Rückgang der privaten Spartätigkeit voraussagen. Zwar sehen auch Cutler et al. Probleme im Zusammenhang mit dem demographischen Wandel, sie sehen aber auch Chancen, insbesondere bei freiem Kapitalverkehr und Produktivitätsfortschritten. Diese Ansicht wird schon im Titel ihrer Arbeit deutlich: "An Aging Society: Opportunity or Challenge?".

\section{Raffelhüschen und Kitterer (1990)}

Die Arbeit von Raffelhüschen und Kitterer ist speziell auf einen Systemwechsel vom Umlage- zum Kapitaldeckungsverfahren in der deutschen Rentenversicherung ausgerichtet. Raffelhüschen und Kitterer nehmen den Ansatz von Auerbach et al. (1989) auf und beschreiben das Sparverhalten der Haushalte ebenfalls mit dem klassischen Lebenszyklusmodell, erweitert um ein Erbschaftsmotiv bei sicherer Lebenserwartung. Nicht überraschend haben Raffelhüschen und Kitterer ähnliche Schwierigkeiten bei der Kalibrierung ihrer Simulation. Zwar verwenden sie eine positive Zeitpräferenzrate, aber das Erbschaftsmotiv ist weiterhin sehr dominant. Gegenüber Auerbach et al. vereinfachen Raffelhüschen und Kitterer ihre Simulation, denn sie fassen die Bevölkerung in sieben Generationen zusammen und nehmen ein konstantes Bevölkerungswachstum an. Die Simulation zeigt, daß ein Systemwechsel zu einer kapitalgedeckten Alterssicherung einen deutlichen Anstieg der gesamtwirtschaftlichen Ersparnis und der Kapitalintensität bewirkt. Getrübt werden diese langfristig positiven Aussichten durch die Wohlfahrtsverluste der Übergangsgeneration, die neben den Ersparnissen für die kapitalgedeckte Alterssicherung auch noch Beiträge zur umlagefinanzierten Rentenversicherung aufbringen muß.

In einer späteren Anwendung untersucht Raffelhüschen (1993), ob ein Umstieg auf eine kapitalgedeckte Alterssicherung möglich ist, bei dem keine Generation schlechter gestellt wird. In dem speziellen Rahmen seines Modells konstruiert Raffelhüschen ein solches pareto-verbesserndes Umstiegsszenario. 
Börsch-Supan (1995c, 1995d und 1996)

Die Simulationen von Börsch-Supan folgen dem Ansatz von Cutler et al. (1990) und untersuchen die Chancen, die sich für das "alternde" Deutschland ergeben, wenn Kapitalexporte in Länder mit einer jüngeren Bevölkerung berücksichtigt werden. Da fast alle westlichen Länder eine mehr oder weniger starke Zunahme der älteren Bevölkerung erfahren werden, stellt Börsch-Supan den OECD-Ländern die südostasiatischen Schwellenländer ${ }^{2}$ gegenüber, die ein hohes Bevölkerungswachstum aufweisen, denn gerade in diesen Ländern besteht ein hoher Kapitalbedarf. Das Kapital, das durch die in den westlichen Ländern abnehmende Anzahl der Erwerbspersonen frei wird, kann in den Schwellenländern sinnvoll die Kapitalintensität erhöhen. Dadurch wird dem starken Absinken der Zinssätze entgegengewirkt, das sich bei einer isolierten Betrachtung der „alternden“ westlichen Länder ergeben würde. Genau diese Hypothese untermauern Börsch-Supans Simulationen. Im Vergleich zur Autarkie eröffnen sich für Deutschland als Teil eines internationalen Kapitalmarkts demnach deutlich höhere Konsummöglichkeiten. Allerdings zeigt Börsch-Supan auch, daß die Kapitalexporte nicht ausreichen, um die negativen Folgen des demographischen Wandels bei einer unveränderten Rentenversicherung völlig abzufangen.

\section{Buslei und Kraus (1996)}

Buslei und Kraus orientieren sich wieder stärker an den Arbeiten von Raffelhüschen und Kitterer (1990) sowie Auerbach et al. (1989). Nach einer ausführlichen theoretischen Diskussion der Vor- und Nachteile von Umlagefinanzierung und Kapitaldeckung simulieren Buslei und Kraus einen allmählichen Übergang auf ein teilweise kapitalgedecktes Alterssicherungssystem. Erreicht wird die Stärkung der kapitalgedeckten Alterssicherung durch eine langsame Absenkung des Rentenniveaus der umlagefinanzierten Rentenversicherung. Auf diese Weise erhöht das Szenario von Buslei und Kraus nicht nur die private Kapitalbildung, sondern beachtet auch ein Urteil des Bundesverfassungsgerichts, wonach schon erworbene Rentenansprüche zumindest teilweise als Eigentum angesehen werden müssen und deshalb nicht entzogen werden dürfen. Buslei und Kraus halten als Ergebnis fest, daß die allmähliche Senkung des Rentenniveaus mittel- und langfristig deutliche Wohlfahrtsgewinne impliziert, denen kurzfristig geringe Wohlfahrtsverluste der Übergangsgeneration gegenüberstehen. Diese Wohlfahrtsverluste sind nach Auffassung von Buslei und Kraus so gering, daß sie ihr Szenario für eine realistische Politikoption halten.

2 Die südostasiatischen Schwellenländer sind Hong Kong, Indonesien, Süd-Korea, Malaysia, die Philippinen, Singapur und Taiwan. 


\subsection{Aufbau der Arbeit}

Die erwähnten Studien sind die wichtigsten Ausgangspunkte meiner eigenen Analyse. Nach den grundlegenden Arbeiten zu Beginn der achtziger Jahre sind inzwischen viele makroökonomische Simulationsstudien zu ganz unterschiedlichen Fragestellungen entwickelt worden. Diese Simulationsstudien haben eine gemeinsame Methodik, die im Journal of Economic Perspectives in drei unterschiedlichen Sichtweisen zur Diskussion gestellt wurde, siehe Kydland und Prescott (1996), Hansen und Heckman (1996) sowie Sims (1996). Der Aufsatz von Kydland und Prescott unterstützt die Arbeit mit Simulationsmodellen in der Makroökonomik und beginnt mit einer kurzen Beschreibung der wesentlichen Elemente einer Simulationsstudie:

"In a computational experiment, the researcher starts by posing a well-defined quantitative question. Then the researcher uses both theory and measurement to construct a model economy that is a computer representation of a national economy. A model economy consists of house-holds, firms and often a government. The people in the model economy make economic decisions that correspond to those of their counterparts in the real world. [...] The researcher then calibrates the model economy so that it mimics the world along a carefully specified set of dimensions. Finally, the computer is used to run experiments that answer the question." (Kydland and Prescott, 1996, p. 69)

Meine Arbeit hält sich an diesen Rahmen. Die well-defined quantitative question untersucht die makroökonomischen Auswirkungen des demographischen Wandels in einer weiterentwickelten Rentenversicherung, speziell die Kapitalintensität, die Sparquote, den Beitragssatz zur Rentenversicherung, das Arbeitsangebot und den Pro-Kopf-Konsum.

Kapitel 2: Sparverhalten der Haushalte

Als Fundament dient das klassische Lebenszyklusmodell von Modigliani und Brumberg (1954), das ich im ersten Abschnitt kurz vorstelle und dabei seine Restriktionen im Zusammenhang mit empirischen Untersuchungen deutlich herausarbeite. Die wesentlichen Erweiterungen des klassischen Lebenszyklusmodells behandelt Abschnitt 2.2, ebenfalls aus theoretischer und empirischer Sicht. In Abschnitt 2.3 versuche ich, das Sparverhalten der Haushalte aus einem ganz anderen Blickwinkel zu betrachten. Der Ansatz nimmt Ideen aus dem Bereich der 
Behavioural Economics ${ }^{3}$ auf und entwickelt ein Mental Accounting ${ }^{4}$ Modell soweit, daß Konsum- und Sparprofile für die Haushalte simuliert werden können. Die Bewertung des Mental Accounting Modells an Hand empirischer Evidenz offenbart interessante Aspekte, die eine weitere Beschäftigung mit Ideen aus dem Bereich der Behavioural Economics interessant erscheinen lassen; sie zeigt aber auch die Schwächen dieses Ansatzes. In Abschnitt 2.4 komme ich zurück zur neoklassischen Theorie und erweitere das Lebenszyklusmodell um einen Erbschaftsnutzen bei unsicherer Lebenserwartung. Gegenüber den in Abschnitt 1.2 erwähnten Arbeiten ist die Berücksichtigung der unsicheren Lebenserwartung ein wesentlicher Fortschritt. Mit diesem „Erbschaftsnutzenmodell“ integriere ich den Haushaltssektor in das allgemeine Gleichgewichtsmodell.

\section{Kapitel 3: Das Simulationsmodell}

Im dritten Kapitel beschreibe ich, wie die einzelnen Teilmodelle in das Simulationsmodell integriert werden. Abschnitt 3.1 schließt sich an das zweite Kapitel an und paßt das Erbschaftsnutzenmodell an die Simulation an: Das Modell wird explizit für die Betrachtung von je einem repräsentativen Haushalt pro Geburtsjahrgang formuliert und das Arbeitsangebot wird endogenisiert. Schließlich spezifiziere ich die Nutzenfunktionen, aus denen sich der Lebensnutzen der Haushalte zusammensetzt.

Abschnitt 3.2 bringt die Bevölkerungsprognose und schafft die Verbindung zwischen den einzelnen Personen und den Haushalten. Ein weiterer wichtiger Punkt ist die Frage der Erbschaftsbeziehungen, die sich bei unsicherer Lebenserwartung nicht nur für ein Alter, sondern für alle Lebensalter stellt und sorgfältig modelliert werden muß.

Den Produktionssektor führe ich in Abschnitt 3.3 ein. Da der Schwerpunkt meiner Arbeit nicht auf dem Firmenmodell liegt, orientiere ich mich an dem stilisierten Modell von Auerbach et al. (1989) und bilde den Produktionssektor mit einer repräsentativen Firma ab.

Wesentlich ausführlicher behandele ich die Rentenversicherung in Abschnitt 3.4, denn diese ist zentral mit dem Thema meiner Arbeit verknüpft. Folglich versucht die Modell-Rentenversicherung das in Deutschland existierende Umlageverfahren so weit wie möglich nachzubilden. Durch entsprechende Wahl der Parameter

3 Eine treffende deutsche Bezeichnung für Behavioural Economics gibt es meiner Meinung nach nicht, daher verwende ich in meiner Arbeit den englischen Ausdruck.

4 Auch Mental Accounting behalte ich als englischen Begriff bei. 
kann die Modell-Rentenversicherung auch in ein Kapitaldeckungsverfahren überführt werden.

Die vier Teilmodelle (Haushaltssektor, Bevölkerungsprognose, Produktionssektor und Rentenversicherung) fasse ich im Abschnitt 3.5 in einem allgemeinen Gleichgewichtsmodell zusammen und erkläre, wie die Teilmodelle zusammenspielen. Die numerische Umsetzung in ein Simulationsprogramm diskutiere ich in Grundzügen, weitergehende Details bringt Anhang A2.

Das Kapitel schließt in Abschnitt 3.6 mit der Kalibrierung, der Sensitivitätsanalyse und der Untersuchung des Konvergenzverhaltens des Simulationsmodells. Ausführlich widme ich mich den Daten, mit denen das Simulationsmodell in bestimmten Dimensionen möglichst gut an die Realität angepaßt werden soll.

\section{Kapitel: Ergebnisse des Simulationsmodells}

Das vierte Kapitel umreißt die Bandbreite möglicher gesamtwirtschaftlicher Entwicklungen durch speziell definierte Szenarien. Abschnitt 4.1 beginnt mit einem Basisszenario, das die heutige Situation weitgehend unverändert fortschreibt. Das Basisszenario verwende ich als Referenzsituation, zu der ich in Abschnitt 4.1 zwei „politikferne“ Szenarien in Beziehung setzte. Diese politikfernen Szenarien untersuchen, wie sich veränderte Mortalitäts- und Fertilitätsentwicklung auf die Modellökonomie auswirken. In Abschnitt 4.2 führe ich vier „politiknahe“ Szenarien ein, die die grundsätzlichen Möglichkeiten der Politik analysieren: erhöhtes Rentenalter, gezielte Einwanderungspolitik, Umstieg auf eine vollständig kapitalgedeckte Alterssicherung sowie allmähliche Senkung der Ersatzquote. Diese Szenarien gehen von einer geschlossenen Volkswirtschaft aus. Die Auswirkungen des demographischen Wandels in einer kleinen offenen Volkswirtschaft untersuche ich in Abschnitt 4.3 mit zwei weiteren Szenarien.

\section{Kapitel: Zusammenfassung und Ausblick}

Im fünften Kapitel fasse ich die Methode und die wichtigsten Ergebnisse der Arbeit noch einmal kurz zusammen. In dem abschließenden Ausblick schlage ich einige Erweiterungen vor, die interessante Ansatzpunkte für weitergehende Forschungen bieten können. 


\section{Sparverhalten der Haushalte}

Das Sparverhalten der Haushalte beeinflußt ganz wesentlich die gesamtwirtschaftliche Entwicklung, denn im idealisierten Fall der geschlossenen Volkswirtschaft ohne Staat entsprechen die aggregierten Ersparnisse genau den aggregierten Investitionen. Produktivitätssteigernde Investitionen müssen deshalb durch die Haushaltsersparnisse gedeckt werden. Wenn die Haushaltsersparnisse durch den demographischen Wandel abnehmen, dann steht weniger Kapital für Investitionen zur Verfügung, so da $\beta$ das langfristige Wachstum der Volkswirtschaft auf Grund zurückgehender Produktivitätssteigerungen abnehmen könnte. Die aggregierten Haushaltsersparnisse entstehen durch das Sparverhalten der einzelnen Kohorten ${ }^{1}$, deren Anteil an der Gesamtbevölkerung durch den demographischen Wandel stark verändert wird. Daher stelle ich bei der Untersuchung der gesamtwirtschaftlichen Auswirkungen des demographischen Wandels das Sparverhalten der Haushalte an den Anfang.

Ausgangspunkt des Kapitels ist das Konsum- und Sparmodell von Modigliani und Brumberg (1954), dessen Grundversion ich im folgenden klassisches Lebenszyklusmodell nenne. Zu Beginn, in Abschnitt 2.1, klärt eine kurze theoretische Darstellung die Begriffe und zeigt, warum das klassische Lebenszyklusmodell das Sparverhalten der Haushalte nur sehr eingeschränkt beschreiben kann, wofür Ergebnisse aus der empirischen Literatur Belege liefern. Die wichtigsten Erweiterungen des klassischen Lebenszyklusmodells sind Thema des zweiten Abschnitts. Einen völlig anderen Weg beschreite ich im dritten Abschnitt, der ein Modell entwickelt, welches das Sparverhalten der Haushalte mit Elementen aus Behavioural Economics beschreibt. Dieser Abschnitt verläßt das Gebiet der Neoklassik. Der neoklassische Ansatz soll aber nicht verworfen werden, vielmehr geht es darum, aus diesem Experiment interessante Ideen herauszufiltern, die möglicherweise in ein neoklassisches Modell übernommen werden können. Für das allgemeine Gleichgewichtsmodell kehre ich im vierten Abschnitt aber in die Neoklassik zurück und bringe eine Erweiterung des klassischen Lebenszyklusmodells: Die Unsicherheit über die Lebenserwartung.

Das Ziel dieses Kapitels ist es, ein Modell für das Sparverhalten der Haushalte zu entwickeln, das flexibler ist als die nur wenig erweiterten Versionen des klassischen Lebenszyklusmodells (die in den eingangs erwähnten Simulationsstudien verwendet werden), das aber trotzdem geeignet ist, Teil eines allgemeinen Gleichgewichtsmodells zu sein. Daraus ergibt sich eine wichtige Forderung: Das Haushaltsmodell muß numerisch zu lösen sein.

1 In dieser Arbeit bildet jeder Geburtsjahrgang eine Kohorte. 
Das Ziel dieses Kapitels ist es nicht, eine umfassende Darstellung der neoklassischen Modelle des Konsum- und Sparverhaltens zu geben, dazu verweise ich auf die Literatur, insbesondere auf Deaton (1992), Browning und Lusardi (1995) sowie King (1985).

\subsection{Klassisches Lebenszyklusmodell}

Ein ganz wesentlicher Gedanke des klassischen Lebenszyklusmodells von Modigliani und Brumberg (1954), aber auch der permanenten Einkommenshypothese von Friedmann (1957), ist der intertemporale Kontext, indem der Konsum erklärt wird. Stellt die Keynesianische Konsumfunktion lediglich einen $\mathrm{Zu}$ sammenhang zwischen dem Konsum und dem laufenden Einkommen her, so verknüpft das Lebenszyklusmodell den gegenwärtigen Konsum mit dem Lebenseinkommen des Haushalts. Unabhängig von aller Kritik im Detail, die man heute gegen das Lebenszyklusmodell vorbringt, ist dieser Gedanke aus der Theorie des Konsum- und Sparverhaltens nicht mehr wegzudenken. Leider ist der Terminus "Lebenszyklusmodell“ nicht eindeutig definiert: Manche bezeichnen damit ein Modell a la Modigliani und Brumberg, andere fassen diesen Begriff so weit, daß er fast alle intertemporalen Konsummodelle umfaßt. Daher verwende ich den Begriff klassisches Lebenszyklusmodell für die Version von Modigliani und Brumberg, die gleich eingeführt wird. Nach dem kurzen theoretischen Teil nimmt die Diskussion der empirischen Studien breiteren Raum ein, denn die zumindest problematische empirische Evidenz für das klassische Lebenszyklusmodell liefert die Motivation für die Modellerweiterungen, die im Abschnitt 2.2 folgen.

\subsubsection{Theoretische Betrachtung}

Die theoretischen Betrachtung beginnt mit einigen Begriffsklärungen:

- Ein Konsumprofil ordnet jedem Zeitpunkt den zugehörigen Konsum zu. Entsprechend sind Arbeitseinkommens- und Vermögensprofil definiert.

- Ein normales Arbeitseinkommensprofil ist gekennzeichnet durch ein nicht fallendes Arbeitseinkommen und ein Renteneinkommen, das niedriger als das letzte Arbeitseinkommen ist.

- Eine Nutzenfunktion hat in dieser Arbeit grundsätzlich abnehmenden Grenznutzen, das heißt eine positive erste Ableitung und eine negative zweite Ableitung. 
Im klassischen Lebenszyklusmodell lebt der Haushalt in einer vollkommen sicheren Welt, in der er ohne Kosten Zugang zu allen relevanten Informationen hat und diese in seiner vorausschauenden, rationalen Planung verwendet. Das Ziel des Haushalts ist es, das Konsumprofil zu finden, das die Summe der abdiskontierten Periodennutzen maximiert. Wenn $u$ den Nutzen aus dem Konsum $c$ eines Jahres $t$ bezeichnet, dann hat die Optimierungsaufgabe des Haushalts eine einfache Zielfunktion:

$$
\max \sum_{t=\tau}^{T}(1+\rho)^{\tau-t} u\left(c_{t}\right)
$$

Die Zielfunktion (2.1) ist der Lebensnutzen, der sich additiv-separabel ${ }^{2}$ aus den Periodennutzen zusammensetzt und die zukünftigen Jahre mit der Zeitpräferenzrate $\rho$ auf das Planungsjahr $\tau$ abdiskontiert. Nach dem Jahr $T$ stirbt der Haushalt. Bei der Optimierung der Zielfunktion muß der Haushalt seine Budgetrestriktion einhalten:

$$
a_{t+1}=(1+r) a_{t}+w_{t}-c_{t} \quad \text { für } t=\tau, \ldots, T
$$

Die Variable $a_{t}$ steht für die Assets des Haushalts zu Beginn des Jahres $t, r$ bezeichnet den Zinssatz (der zur Vereinfachung zunächst konstant ist) und $w$ ist das Arbeitseinkommen. Zusätzlich zu der Budgetrestriktion, die hier als Bewegungsgleichung angegeben ist, müssen noch Anfangs- und Endbedingungen für die Asset festgelegt werden: Die einfachste Annahme ist, daß der Haushalt sein Leben ohne Assets beginnt und beendet. Damit ist die Optimierungsaufgabe vollständig gegeben. Das Maximumprinzip ${ }^{3}$ löst die Aufgabe und ergibt als Optimalitätsbedingung die Eulergleichung:

$$
\frac{\partial u\left(c_{t+1}\right)}{\partial c_{t+1}}=\frac{(1+\rho)}{(1+r)} \frac{\partial u\left(c_{t}\right)}{\partial c_{t}} \quad \text { für } t=\tau, \ldots, T-1
$$

Die Eulergleichung verdeutlicht den Unterschied zur Keynesianischen Konsummodell: Das Einkommen eines Jahres hat keinen direkten Einfluß auf den nutzenmaximierenden Konsum dieses Jahres, es wirkt lediglich mittelbar über das Lebenseinkommen auf das Niveau des Konsumprofils. Die Steigung des optima-

2 Für eine ausführliche Diskussion der Separabilität verweise ich auf Deaton und Muellbauer (1980).

3 Eine hervorragende Einführung in die Optimierung ist Dixit (1990): Optimization in Economic Theory. 
len Konsumprofils - also sein Verlauf - ist völlig unabhängig vom Budget, sie hängt allein von den Präferenzen $u$, dem Zinssatz $r$ und der Zeitpräferenzrate $\rho$ ab. Wenn Zeitpräferenzrate und Zinssatz gleich sind, dann müssen im Optimum die marginalen Konsumnutzen in allen Jahren gleich sein - also konsumiert der Haushalt in allen Jahren gleich viel. Abbildung 2.1 zeigt den entsprechenden Verlauf des Konsumprofils für einen Haushalt, der während seines Arbeitslebens ein konstantes Arbeitseinkommen bezieht und danach eine geringe Rente bekommt. Das zugehörige Vermögensprofil hat den für das klassische Lebenszyklusmodell charakteristischen buckelförmigen (hump shaped) Verlauf. Wenn Zinssatz und Zeitpräferenzrate nicht gleich sind, ändern sich die Profile nur unwesentlich. Zum Beispiel ist die Zeitpräferenzrate von ungeduldigen Haushalten größer als der Zinssatz, daher konsumieren ungeduldige Haushalte am Anfang ihres Lebens mehr als am Ende; das entsprechende Konsumprofil fällt mit konstanter Rate, das Vermögensprofil behält seinen buckelförmigen Verlauf. Im klassischen Lebenszyklusmodell hat das Vermögen nur eine Funktion: Es dient der Konsumglättung. Wenn ein normales Einkommensprofil gegeben ist, dann spart der Haushalt während des Arbeitslebens und "entspart" nach der Verrentung.

Abbildung 2.1: Konsum-, Arbeitseinkommens- und Vermögensprofil im klassischen Lebenszyklusmodell

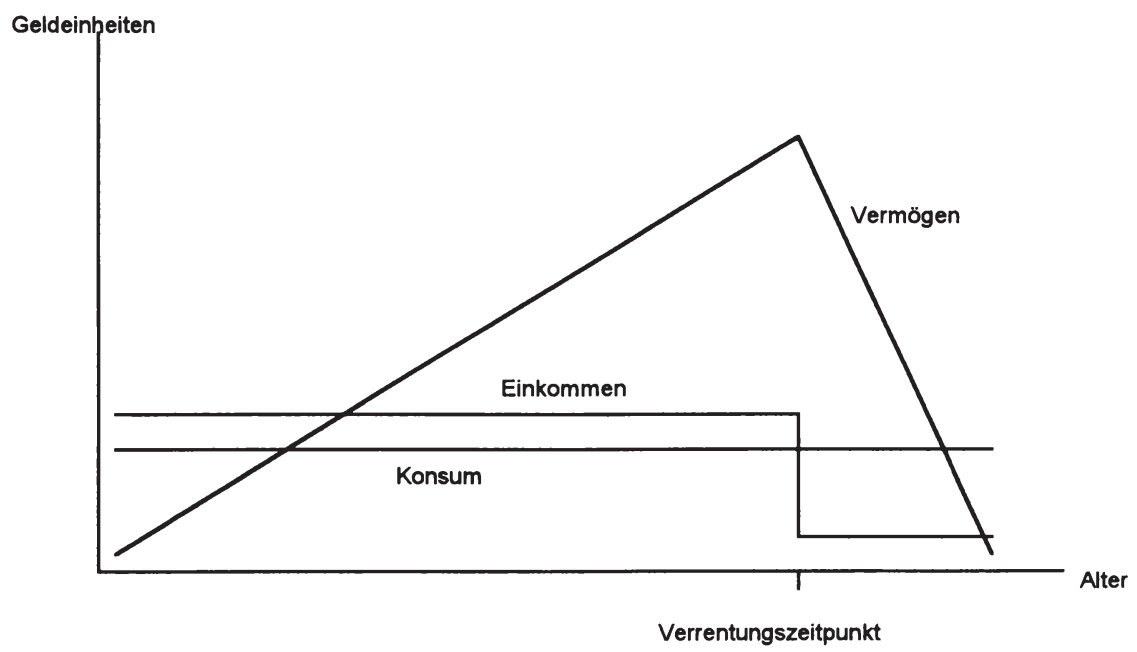

Quelle: nach Deaton (1992) 
Die Eulergleichung charakterisiert das klassische Lebenszyklusmodell durch zwei wichtige Aussagen, die völlig unabhängig von der Parametrisierung der Präferenzen, dem Einkommensprofil und dem Verhältnis zwischen Zinssatz und Zeitpräferenzrate sind:

- Die Steigung des Konsumprofils, d.h. die intertemporale Allokation des Konsums, ist unabhängig vom Einkommensprofil, denn die Eulergleichung verbindet nur die marginalen Konsumnutzen mit dem Verhältnis von Zeitpräferenzrate und Zinssatz.

- Das Lebenseinkommen bestimmt das Niveau des Konsumprofils. Das Einkommen einzelner Jahre hat nur über das Lebenseinkommen Einfluß auf den Konsum, daher ist die Korrelation zwischen dem Einkommen und dem Konsum eines Jahres gering.

Aus diesen beiden Kernaussagen ergibt sich eine dritte Aussage: Das Vermögen dient ausschließlich der Konsumglättung. Wenn ein normales Arbeitseinkommensprofil gegeben ist, dann hat das Vermögensprofil einen buckelförmigen Verlauf.

\subsubsection{Empirische Evidenz - Ergebnisse aus der Literatur}

Die aus der Theorie abgeleiteten eindeutigen Aussagen des klassischen Lebenszyklusmodells sollten sich eigentlich leicht empirisch überprüfen lassen. In der Tat wurden auch große Anstrengungen unternommen, um das Modell empirisch zu untermauern oder abzulehnen, doch noch immer ist das Lebenszyklusmodell $A n l a ß$ heftiger Kontroversen. Ein Streitpunkt ist die unscharfe Abgrenzung des Begriffs „Lebenszyklusmodell“. Ein anderes, wichtigeres Problem ist die korrekte Erfassung der Ersparnisse, die sich prinzipiell auf zwei verschiedene Weisen definieren lassen: einerseits als Differenz aus dem Vermögen in aufeinanderfolgenden Zeitpunkten, andererseits als Differenz zwischen dem Konsum und der Summe aus Arbeits- und Zinseinkommen. In der Theorie sind beide Definitionen identisch, denn es sind die beiden Seiten der Budgetrestriktion (2.2), aber in der Empirie können sie stark voneinander abweichen.

Wenn die Ersparnis $s$ im Jahr $t$ als Differenz zwischen dem Vermögen zu Beginn des Jahres $t$ und dem Vermögen zu Beginn des Vorjahres $t-1$ gemessen wird, dann besteht der folgende Zusammenhang:

$$
s_{t}=a_{t}-a_{t-1}
$$

Das Problem dieser Definition liegt in der Erfassung des Vermögens: Wie sollen langlebige Konsumgüter berücksichtigt werde? Sind Rentenansprüche Teil des 
Vermögens? Wie werden Immobilien in die Konsum- und Vermögenskomponente getrennt? Werden Wertsteigerungen der Immobilien korrekt dargestellt? Lassen sich Kursänderungen von Wertpapieren, die schon im Portfolio gehalten werden, richtig erfassen? Kann man überhaupt erwarten, wahrheitsgemäße Vermögensangaben von den Haushalten zu bekommen? Die Fragen machen deutlich, warum die Ersparnisse nach Definition (2.4) empirisch nur schwer abzugrenzen sind, denn selbst wenn es präzise Vermögensdaten gäbe, bliebe noch viel Spielraum für die Aggregation der einzelnen Vermögenstitel zu der Variablen $a$.

Die zweite Definition der Ersparnis bestimmt die Differenz aus dem Zins- und Arbeitseinkommen und dem Konsum eines Jahres:

$$
s_{t}=r_{t-1} \cdot a_{t-1}+w_{t-1}-c_{t}
$$

Das Arbeitseinkommen ist relativ genau zu erfassen, problematisch sind das Zinseinkommen und der Konsum. Auf das Zinseinkommen übertragen sich die gerade erwähnten Probleme der Vermögensmessung. Eine korrekte Erfassung des Konsums nach Definition (2.5) muß sowohl langlebige Konsumgüter als auch den Konsumanteil von Immobilien berücksichtigen. Weil beide Fragen nicht geklärt sind, bietet auch die zweite Definition viele Möglichkeiten für die Erfassung der Ersparnisse.

Neben der Erfassung der Ersparnisse gibt es noch weitere Schwierigkeiten bei der empirischen Arbeit. Die relevanten Datensätze enthalten meistens Informationen über Haushalte, so daß die Haushaltszusammensetzung Bedeutung hat. Zum Beispiel könnte ein Haushalt aus drei jungen Mitgliedern und einem 65-jährigen Haushaltsvorstand bestehen; ein anderer Haushalt könnte ebenfalls einen 65-jährigen Haushaltsvorstand haben und aus drei weiteren Mitgliedern im Rentenalter bestehen. Je nach Erwerbsbeteiligung und Einkommenshöhe der einzelnen Haushaltsmitglieder ergeben sich für verschiedene Haushalte völlig unterschiedliche Einkommens- und Konsumaufteilungen innerhalb des Haushalts. Neben diesem Problem wird an dem Beispiel ein weiterer Punkt deutlich: Das Alter des Haushaltsvorstandes sagt nur wenig über die Zusammensetzung des Haushalts aus. Nun entscheidet das Alter des Haushaltsvorstands in der Regel über die Zuordnung des Haushalts zu einer Kohorte. Aber wer ist der Haushaltsvorstand? Ist es das älteste Mitglied? Oder ist es das Mitglied mit dem höchsten Arbeitseinkommen? Oder entscheidet der Haushalt nach irgendwelchen anderen Kriterien, wer Haushaltsvorstand genannt werden soll? Diese Abgrenzung ist wichtig aber leider unpräzise, denn das „Haushaltsalter“ ist entscheidend bei der Trennung von Alters- und Kohorteneffekten. Damit ergibt sich ein weiteres Problem: Aus Querschnittsdaten kann man nichts über Alters- und Kohorteneffekt lernen, denn dafür sind Paneldaten notwendig. Gute Paneldaten haben aber oft noch zu wenig Wel- 
len, so daß viele Studien aufeinanderfolgende Querschnittsdaten (repeated cross sections) zu einem synthetischen Panel zusammensetzen, womit sich Alters- und Kohorteneffekt zumindest bedingt trennen lassen. Der Nachteil von synthetischen Paneldaten ist der gegenüber echten Paneldaten reduzierte Informationsgehalt, denn synthetische Panel sind keine Individualdaten, weil sie die einzelnen Haushalte nach bestimmten Merkmalen zu Gruppen zusammenfassen. Durch diese Mittelwertbildung gehen individuelle Eigenschaften der Haushalte verloren, die Heterogenität der individuellen Haushalte wird durch die Gruppenbildung geglättet.

Die geschilderten Schwierigkeiten bei empirischen Arbeiten bestimmen ganz wesentlich die Kontroverse über das Lebenszyklusmodell. Gerade weil die entscheidenden Definitionen großen Raum für Variationen lassen, stehen die empirischen Ergebnisse auf tönernen Füßen, so daß die Diskussion oft mehr von ideologischen als sachlichen Gesichtspunkten geprägt ist. Dennoch versuche ich in der folgenden Zusammenstellung einiger empirischer Arbeiten sachlich zu argumentieren und die für meine Arbeit wesentlichen Punkte herauszuarbeiten.

\section{Empirische Evidenz: Vermögensprofil}

Die Frage, ob die Haushalte gemäß dem Lebenszyklusmodell ihr Vermögen im Alter abbauen oder nach der Verrentung weiter sparen, führt auch nach zwanzig Jahren Forschung zu scharfen Diskussionen. Mirer (1979) analysiert Querschnittsdaten und findet zunächst ein im Alter fallendes Vermögensprofil, womit das Lebenszyklusmodell unterstützt wäre. In einem zweiten Schritt kontrolliert er für Unterschiede zwischen den Kohorten und stellt fest, daß dann der buckelförmige Verlauf des Vermögensprofils verschwindet; nun steigt das Vermögen mit dem Alter an. Danziger et al. (1982) finden sogar die höchsten Sparquoten für die ältesten Alten, unabhängig von deren Position in der Einkommensverteilung. Die Zweifel an dem buckelförmigen Vermögensverlauf werden durch Hall und Mischkin (1982) erhärtet, die ihre Studie auf ein Haushaltspanel gründen. Auch sie finden mit zunehmendem Alter steigendes Vermögen. $\mathrm{Zu}$ anderen Ergebnissen kommt Hurd (1987, 1990), der das Lebenszyklusmodell vehement verteidigt. Seine Panelstudie weist für die Älteren ein abnehmendes Vermögen aus - allerdings betrachtet Hurd nur Ältere bis 70 Jahre. Der Teil der Älteren, für den Danziger et al. die höchsten Sparquoten ausweisen, wird in Hurds Studie folglich nicht abgebildet. Hurds Argument gegen die steigenden Vermögensprofile im Alter beruht auf der beobachteten differentiellen Mortalität: Im Durchschnitt werden reichere Personen älter als Ärmere. Sind die Unterschiede in der Mortalität groß genug, können sich bei einer Kohortenbetrachtung, die alle Haushalte eines Jahrgangs zusammenfaßt, auch nach der Verrentung steigende Vermögensprofile ergeben. Börsch-Supan und Stahl (1991) nehmen Hurds Argument auf. Obwohl 
sie für die Mortalität kontrollieren, können sie das Ergebnis von Danziger et al. für Deutschland bestätigen. Börsch-Supan und Stahl finden die höchsten Sparquoten bei den über Achtzigjährigen und erklären ihre Beobachtung mit zunehmend eingeschränkten Konsummöglichkeiten der älteren Menschen.

Empirische Evidenz: Einkommen und intertemporale Allokation

Das klassische Lebenszyklusmodell kennt keinen Zusammenhang zwischen dem Einkommen und der intertemporalen Allokation des Konsums4: Die Form des Konsumprofils ist für alle Einkommensklassen gleich; die Profile unterscheiden sich lediglich in ihrem Niveau. Gerade diese Aussage ist empirisch kaum nachzuweisen. Bosworth et al. (1991) analysieren US-Haushalte und finden für das unterste Einkommensquartil ein sehr flaches Vermögensprofil; das Vermögen kann höchstens die Konsumausgaben weniger Monate finanzieren. Ähnliche Ergebnisse für Deutschland gewinnt Börsch-Supan (1994) auf Basis der Einkommens- und Verbrauchsstichprobe (EVS) und Börsch-Supan (1995b) bestätigt das heterogene Verhalten in einer expliziten Analyse der Alters- und Kohorteneffekte. Wolf (1995) untersucht ebenfalls die EVS und trennt die Haushalte in Mieter und Eigentümer. Damit grenzt sie zwei Einkommensklassen voneinander ab und kann zeigen, daß sich deren Vermögensprofile nicht nur in Niveau, sondern auch im Verlauf deutlich unterscheiden. Alessie et al. (1995) zeigen für ein niederländisches Haushaltspanel, daß gerade bei den älteren Haushalten die Ungleichheit in der Vermögensverteilung noch zunimmt. Die beobachteten Unterschiede zwischen verschiedenen Einkommensklassen kann das klassische Lebenszyklusmodell nicht erklären. Es ist zu unflexibel, es zwingt das Konsumprofil aller Haushalte in die gleiche Form.

Empirische Evidenz: Eulergleichung

Neben dem Vergleich von theoretisch vorhergesagten Konsum- und Vermögensprofilen mit empirisch ermittelten Profilen kann auch die Eulergleichung (2.3) getestet werden. Diese „indirekte“ Testmethode wurde vor allem im Zusammenhang mit der Theorie der rationalen Erwartungen entwickelt. Wenn der erwartete Lebensnutzen maximiert wird, dann wird die Eulergleichung (2.3) stochastisch:

$$
E_{t}\left[\frac{1+r}{1+\rho} \frac{u^{\prime}\left(c_{t+1}\right)}{u^{\prime}\left(c_{t}\right)}\right]=1
$$

4 Hier ist natürlich die Annahme identischer Präferenzen entscheidend. 
Bei rationalen Erwartungen berücksichtigen die Leute bei ihren Planungen in Periode $t$ alle zu diesem Zeitpunkt bekannten relevanten Informationen. Da sie das auch in der Vorperiode $t$ - 1 getan haben, sollten alle länger zurückliegenden Variablen schon bei der Entscheidung über $c_{t-1}$ eingeflossen sein. Also sollte $c_{t-1}$ die relevanten Informationen der Vergangenheit enthalten. Dies ist der Grundgedanke der bahnbrechenden Arbeit von Hall (1978). Er untersucht, ob Variablen aus der Vergangenheit bei gegebenem $c_{t-1}$ einen Beitrag zur Vorhersage von $c_{t}$ leisten. Nach der Theorie dürfte dies nicht der Fall sein. Tatsächlich zeigt Halls Untersuchung einen insignifikanten Einfluß des vergangenen Einkommens. Dennoch lehnt Hall das Lebenszyklusmod\#ell ab, denn vergangene Änderungen in den Aktienkursen haben einen Erklärungswert für die Konsumentwicklung.

Eine weitere wichtige Arbeit zum Lebenszyklusmodell bei rationalen Erwartungen stammt von Flavin (1981). Ihr Ansatz geht ebenfalls von der Eulergleichung (2.6) aus. Wenn Zinssatz und Zeitpräferenzrate gleich sind, dann müssen Konsumänderungen im Lebenszyklusmodell auf nicht vorhergesehene Änderungen der Erwartungen zurückgeführt werden. Eine erwartete Einkommensänderung dürfte demnach keine Veränderung des Konsums hervorrufen. Dagegen verändert eine unerwartete Einkommensänderung die relevante Informationsmenge und in diesem Fall wird eine Neuplanung des Konsums durch die Theorie gedeckt. Flavin schätzt nun einen Einkommensprozeß und regressiert die Konsumveränderungen auf lagged changes des Einkommensprozesses. Gilt das Lebenszyklusmodell, dann sollte diese Regression keinen Erklärungswert haben, denn bei rationalen Erwartungen kennen die Leute den Einkommensprozeß. Flavins Regression findet aber einen signifikanten Einfluß der lagged changes des Einkommensprozesses, folglich verwirft sie das Lebenszyklusmodell. Ihre Arbeit prägte den Begriff excess sensitivity, mit dem gemessen wird, wie stark der Konsum auf vorhersehbare Änderungen des Einkommens reagiert.

Nach den beiden grundlegenden Arbeiten von Hall und Flavin haben eine Vielzahl anderer Arbeiten das Lebenszyklusmodell ,indirekt" mit der Eulergleichung überprüft. Browning und Lusardi (1995) stellen die wichtigsten dieser Studien in einer Tabelle sehr übersichtlich zusammen.

Empirische Evidenz: Internationale Vergleiche

International vergleichenden Studien bringen weitere Argumente gegen das klassische Lebenszyklusmodell, wie der von Poterba (1994) herausgegebene Sammelband zeigt. Wenn die Ersparnis hauptsächlich zur Sicherung des Konsumniveaus im Alter dient, dann sollten Länder mit einer gut ausgebauten staatlichen Rentenversicherung eine niedrigere aggregierte Sparquote aufweisen als Länder, in denen die Alterssicherung nicht im gleichen Maß vom Staat organisiert wird. 
Tatsächlich zeigt der internationale Vergleich das Gegenteil: Die nordeuropäischen Länder mit ihren umfassenden staatlichen Rentenversicherungen haben deutlich höhere Sparquoten als die USA, deren Rentenversicherung weit weniger großzügig ist.

\subsection{Erweiterungen des klassischen Lebenszyklusmodells}

\subsubsection{Erbschaftsmotiv}

In den achtziger Jahren konzentrierte sich die Kritik am klassischen Lebenszyklusmodell vor allem auf den Vermögensverlauf. Die Diskussion in Abschnitt 2.1 zeigt, daß wichtige Studien gerade für ältere Haushalte steigende Ersparnisse beobachten und damit im Widerspruch zur Theorie stehen. Für diese Beobachtung bietet das klassische Lebenszyklusmodell kein Motiv, weil das Vermögen dort einzig und allein der Konsumglättung dient. Eine naheliegende Begründung für positives Vermögen am Lebensende ist ein Erbschaftsmotiv. Ob altruistische Gründe Ursache des Erbschaftsmotivs sind oder ob ein strategisches Erbschaftsmotiv ${ }^{5}$ nach Bernheim et al. (1985) unterstellt wird, ist in seiner Konsequenz von untergeordneter Bedeutung, denn beide ordnen dem Vermögen einen Nutzen zu, der von der Konsumglättung unabhängig ist. In einer grundlegenden theoretischen Arbeit hat Hurd (1989) gezeigt, daß auch das Vererbungsmotiv ab einem bestimmten Alter zu abnehmendem Vermögen führt - solange die Zeitpräferenzrate den Zinssatz übersteigt, Separabilität zwischen Konsum und Erbschaft vorausgesetzt wird und ein konstanter marginaler Erbschaftsnutzen angenommen wird. Also kann das Erbschaftsmotiv in Hurd's Modell das beobachtete positive Vermögen im hohen Alter erklären - es kann aber nicht erklären, warum gerade bei den über achtzigjährigen Haushalten zunehmendes Vermögen beobachtet wird.

\section{Inter vivos Transfers}

Wenn das Erbschaftsmotiv auf Altruismus beruht, dann gibt es gute Gründe, schon zu Lebzeiten Vermögen auf die Erben zu übertragen. Tatsächlich findet Hurd (1987) deutliche Hinweise auf inter vivos Transfers, zu denen auch die Bildungsausgaben gerechnet werden können, aber wenig Evidenz für geplante Erbschaften am Lebensende. Er gewinnt dieses Ergebnis aus dem Vergleich von Haushalten mit und ohne Kindern. In seiner Schlußfolgerung führt Hurd die beob-

5 Ein strategisches Erbschaftsmotiv liegt vor, wenn der Erblasser die Erbschaft als Druckmittel einsetzt, um so ein bestimmtes Verhalten des Erben zu erzwingen. 
achteten Erbschaften am Lebensende auf die Unsicherheit über den Todeszeitpunkt zurück - ein Risiko, das auf einem unvollständigen Annuitätenmarkt nicht adäquat versichert werden kann. Er nennt diese Erbschaften Accidental Bequests.

Für Deutschland gibt es nur wenige empirische Untersuchungen über inter vivos Transfers. Auswertungen der Berliner Altersstudie, siehe Motel und Spieß (1995) sowie Wagner et al. (1996), ergeben aber auch für Deutschland deutliche Hinweise auf inter vivos Transfers. So stellen Motel und Spieß fest, daß Transfers von Eltern an ihre Kinder vor allem dann erfolgen, wenn sich die finanzielle Situation der Kinder schwierig gestaltet, beispielsweise bei Arbeitslosigkeit.

\section{Gesamtwirtschaftliche Bedeutung intergenerationaler Transfers}

Die Bedeutung intergenerationaler Transfers wird nicht nur aus mikroökonomischer, sondern auch aus makroökonomischer Perspektive untersucht. Eine heftige Kontroverse dreht sich um den Anteil des Transfervermögens am aggregierten Nettovermögen der USA. Auf der einen Seite stehen die Untersuchungen von Kotlikoff und Summers (1981) sowie Kotlikoff (1988), die $80 \%$ des aggregierten Nettovermögens der USA auf intergenerationale Transfers zurückführen. Auf der anderen Seite steht Modigliani (1988), der den Anteil des Transfervermögens auf 20 \% schätzt. Ähnlich wie bei der Debatte über das klassische Lebenszyklusmodell6 spielt auch hier die Definition des Transfervermögens eine große Rolle. Zwei Punkte verdeutlichen das Problem:

- Das (Zins-) Einkommen aus bereits erhaltenen Transfers ist ein Streitpunkt. Kotlikoff und Summers betrachten es als Teil der Transfers, Modigliani betrachtet nur die Transfers als solche.

- Die Ausgaben für volljährige Kinder, die noch im elterlichen Haushalt leben, werden von Kotlikoff und Summers als Transfers, aber von Modigliani als Konsum erfaßt. Da diese Ausgaben typischerweise die Kosten für die Schule, die Ausbildung oder das Studium beinhalten, kann es sich um erhebliche Beträge handeln.

Gale und Scholz (1994) greifen die Frage nach der gesamtwirtschaftlichen Bedeutung der intergenerationalen Transfers erneut auf und versuchen, die inter vivos Transfers von den Erbschaften, die beim Tod des Erblassers entstehen, zu trennen. Ihre Untersuchung ergibt für die inter vivos Transfers einen Anteil von $20 \%$ und für die Erbschaften einen Anteil von $30 \%$ am aggregierten Nettover-

6 Nicht zufällig verlaufen die Fronten in der Auseinandersetzung über das Lebenszykhusmodell ähnlich: Kotlikoff und Summers befinden sich auf der anderen Seite als Modigliani. 
mögen der USA. Insgesamt kommen Gale und Scholz auf einen Anteil von $50 \%$ und liegen damit genau zwischen den Schätzungen von Kotlikoff (1988) und Modigliani (1988). Dies belegt die Bedeutung der intergenerationalen Transfers für die aggregierte Ersparnis, denn selbst Modiglianis Schätzung beläuft sich auf $20 \%$ - keinesfalls eine marginale Größe.

Die gesamtwirtschaftliche Bedeutung der intergenerationalen Transfers in Deutschland ist empirisch bisher kaum untersucht. Die Deutsche Bundesbank (1993) geht aber davon aus, daß sich allein die im Erbgang übertragenen Finanzund Sachvermögen zwischen $100 \mathrm{Mrd}$. DM und $200 \mathrm{Mrd}$. DM pro Jahr bewegen. Im Vergleich zum verfügbaren Einkommen im Jahr 1993 von 1.833 Mrd. DM sind das zwischen $5 \%$ und $11 \%$, auch das ist keine marginale Größe.

\section{Kritische Würdigung}

Mikroökonomische Untersuchungen der intergenerationalen Transfers sind besonders für Deutschland noch Mangelware. Dagegen zeigen makroökonomische Analysen, daß intergenerationale Transfers einen erheblichen Anteil des aggregierten Nettovermögens ausmachen. Vielleicht scheitert die mikroökonomische Absicherung des Erbschaftsmotivs an den restriktiven Annahmen des klassischen Lebenszyklusmodells. Gale und Scholz (1994) liefern dafür einen deutlichen Hinweis: Sie zeigen, daß nur eine Minderheit von wohlhabenden Haushalten nennenswerte intergenerationale Transfers leistet. Zu der gleichen Feststellung kommen auch Motel und Spieß (1995) auf Grund der Berliner Altersstudie. Diese Beobachtung macht erneut deutlich, daß systematische Unterschiede im Verhalten von Haushalten mit unterschiedlichem Einkommen bestehen. Das klassische Lebenszyklusmodell kann solche Unterschiede nicht erklären. Ein Erbschaftsmotiv bei unsicherer Lebenserwartung kann diesen Mangel beseitigen, wie Abschnitt 2.4 zeigen wird, und darüber hinaus zur Erklärung der auf makroökonomischer Ebene doch gewichtigen Indizien für intergenerationale Transfers beitragen.

\subsubsection{Liquiditätsrestriktionen}

Liquiditätsrestriktionen treten im klassischen Lebenszyklusmodell nicht auf, denn dort gibt es perfekte Kapitalmärkte. Wenn die Kapitalmärkte nicht vollkommen sind, dann können sehr schnell Liquiditätsrestriktionen auftreten, wie Stiglitz und Weiss (1981) in einem mikroökonomischen Modell zeigen, das von Informationsasymmetrien ausgeht. Die praktischen Konsequenzen sind aus dem tatsächlichen Leben bekannt: Eine Studentin, auch wenn sie gute Karriereaussichten hat, wird kaum eine Bank finden, die ihr einen Kredit auf ihr zu erwartendes Lebenseinkommen einräumt, also ist sie liquiditätsbeschränkt. 
Das klassische Lebenszyklusmodell läßt sich relativ einfach um Liquiditätsbeschränkungen erweitern. Dazu wird neben der Budgetrestriktion (2.2) eine Liquiditätsrestriktion ${ }^{7}$ eingeführt:

$$
a_{t} \geq 0 \quad \text { für } t=\tau, \ldots, T
$$

Die Restriktion fordert, daß das Vermögen zu keinem Zeitpunkt negativ ist. Bei der Optimierung der Lebensnutzenfunktion ist die zusätzliche Restriktion mit Hilfe des Kuhn-Tucker Multiplikators ${ }^{8} \mu_{\uparrow}$ zu berücksichtigen. Als Optimalitätsbedingung ergibt sich:

$$
\frac{\partial u\left(c_{t+1}\right)}{\partial c_{t+1}}=\frac{(1+\rho)}{(1+r)} \frac{\partial u\left(c_{t}\right)}{\partial c_{t}}-\mu_{t} \quad \text { für } t=\tau, \ldots, T-1
$$

Solange das Vermögen positiv ist, verschwindet der Kuhn-Tucker Multiplikator. In diesem Fall unterscheidet sich die Eulergleichung (2.8) nicht von der Eulergleichung (2.3) des klassischen Lebenszyklusmodells. Wenn die Restriktion greift, d.h. das Vermögen null ist, dann ist der Multiplikator $\mu_{4}$ größer als null und das Konsumprofil weicht vom Konsumprofil des klassischen Modells ab: Der marginale Konsumnutzen in Periode $t+1$ ist größer, folglich muß der Haushalt seinen Konsum einschränken. Ist die Kreditrestriktion über mehrere aufeinanderfolgende Perioden bindend, ergibt sich ein enger Zusammenhang zwischen dem Konsum- und Einkommenswachstum, der die excess sensitivity des Konsums erklärt. Das Ergebnis hat weitreichende Konsequenzen, denn jetzt bestimmt das Einkommen nicht nur das Niveau, sondern auch die intertemporale Allokation des Konsums. Zwei der drei Kernaussagen des klassischen Lebenszyklusmodells werden dadurch aufgehoben. Das Modell mit Liquiditätsrestriktionen

- stellt eine Verbindung zwischen der intertemporalen Allokation des Konsums und dem Einkommen her

- und kann so für liquiditätsbeschränkte Haushalte eine hohe Korrelation zwischen dem Konsum und Einkommen einer Periode erklären.

Unverändert bleibt die dritte Kernaussage des klassischen Lebenszyklusmodells:

7 Eigentlich handelt es sich um eine Kreditrestriktion. Weil sich in der Literatur die Bezeichnung Liquiditätsrestriktion durchgesetzt hat, schließe ich mich der üblichen Sprachregelung an und spreche von Liquiditätsrestriktion.

8 Für eine Einführung in die Optimierung unter Nebenbedingung verweise ich erneut auf Dixit (1990). 
- Die Ersparnisse dienen ausschließlich der Glättung des Konsums und gehen im Alter zurück.

Also können Liquiditätsrestriktionen die beobachtete Vermögenszunahme im Alter nicht erklären.

\section{Empirische Evidenz}

Zwei Arbeiten, die explizit auf der Eulergleichung (2.8) aufbauen, liefern empirische Evidenz für Liquiditätsrestriktionen und verfolgen eine auch methodisch interessante Idee. Zeldes (1989) verwendet US Haushalte aus dem PSID (PanelStudy of Income Dynamics) und teilt sie nach dem Verhältnis von finanziellem Vermögen zum Einkommen in zwei Gruppen ein. Nach dem theoretischen Modell, der Eulergleichung (2.8), sollten sich die ärmeren und reicheren Haushalte deutlich voneinander unterscheiden: Bei einem hohen Verhältnis von Vermögen zu Einkommen sind Liquiditätsrestriktionen unwahrscheinlich, folglich sollte ein insignifikantes $\mu$ geschätzt werden. Für die Gruppe der ärmeren Haushalte sollte sich dagegen ein signifikant positives $\mu$ ergeben. Tatsächlich kann Zeldes diese Aussage des Modells empirisch untermauern, woraus er schließt, daß Liquiditätsbeschränkungen für einen Teil der Haushalte ein reales Problem darstellen.

Eine ähnliche Idee verfolgen Jappelli und Pagano (1989), indem sie verschiedene Länder vergleichen. Die Schätzwerte für $\mu$ sollten in den Ländern signifikant sein, in denen der Kapitalmarkt durch institutionelle Regelungen stark reglementiert ist. Auch die Untersuchung von Jappelli und Pagano ergibt das vermutete Ergebnis und liefert Argumente für Liquiditätsbeschränkungen.

\section{Kritische Würdigung}

Für einen Teil der Haushalte ist die Liquidität eine echte Beschränkung, zumindest wenn sie zum unteren Einkommensquartil gehören. Liquiditätsrestriktionen können problemlos in den theoretischen Rahmen des klassischen Lebenszyklusmodells integriert werden. Schwierigkeiten gibt es erst, wenn die Eulergleichung (2.8) numerisch gelöst werden muß, denn der Kuhn-Tucker Multiplikator zerstört den stetigen Zusammenhang zwischen $c_{t}$ und $c_{t+1}$ in der Eulergleichung (2.8). Folglich heißt die Aufgabe, eine nichtlineare, unstetige Gleichung numerisch zu lösen. Entsprechende Algorithmen gibt es, allerdings ist deren Effizienz gering. Da sich ein typischer Lebenszyklus über 75 Jahre erstreckt, ist das optimale Konsumprofil ein Vektor mit 75 Dimensionen, so daß heuristische Verfahren schnell an ihre Grenzen stoßen. Gerade im Rahmen eines intertemporalen allgemeinen Gleichgewichtsmodells ist die numerische Effizienz ein limitierender Faktor. 


\subsubsection{Unsicherheit}

Die bisher betrachteten Modelle der Haushaltsersparnis gehen von einer vollkommen sicheren Welt aus. Dagegen spielt Unsicherheit im realen Leben eine große Rolle: Das zukünftige Einkommen, die Lebenserwartung, die Zinssätze und viele Ereignisse im Lebenszyklus sind Beispiele für Variablen, die nicht mit Sicherheit vorhergesagt werden können. ${ }^{9}$ Im Gegensatz zum Erbschaftsmotiv und Liquiditätsrestriktionen erfordert Unsicherheit ein theoretisch anspruchsvolleres Modell. Im folgenden beschreibe ich eine einfache Version des Modells, in der das Einkommen die einzige Quelle der Unsicherheit ist; dabei lehne ich mich teilweise an das Modell von Blanchard und Mankiw (1988) an. Mein Ziel ist es, die Mechanik des Modells herauszuarbeiten.

Sobald die Haushalte eine Optimierungsaufgabe mit unsicheren Größen zu lösen haben, müssen sie Erwartungen über die unsicheren Größen bilden. Dabei wird angenommen, daß die Haushalte auf alle zum Planungszeitpunkt $\tau$ verfügbaren Informationen zurückgreifen und diese in ihre Erwartungen einbeziehen. Bei Unsicherheit maximieren die Haushalte den erwarteten Lebensnutzen. Wenn $E_{t}$ der Erwartungsoperator ist, der alle zum Planungszeitpunkt $\tau$ vorhandenen Informationen berücksichtigt, dann ergibt sich die Eulergleichung (2.6), die ich hier noch einmal angebe:

$$
E_{t}\left[\frac{\partial u\left(c_{t+1}\right)}{\partial c_{t+1}}\right]=\frac{(1+\rho)}{(1+r)} \frac{\partial u\left(c_{t}\right)}{\partial c_{t}} \quad \text { für } t=\tau, \ldots, T-1
$$

Die Eulergleichung beschreibt den optimalen Zusammenhang zwischen dem marginalen Nutzen der Periode $t$, deren Größen bereits realisiert und daher mit Sicherheit bekannt sind, und dem erwarteten marginalen Nutzen der folgenden Periode. Um den optimalen Konsum in Periode $t+1$ bestimmen zu können, muß die Eulergleichung (2.9) nach $E_{t}\left[c_{t+1}\right]$ aufgelöst werden. Da der Erwartungsoperator linear ist, aber die Nutzenfunktion in der Regel nichtlineare Ableitungen hat, ist die analytische Auflösung der Eulergleichung normalerweise nicht möglich. Als Ausweg kann der marginale Nutzen durch eine Taylor-Entwicklung zweiten Grades in der Umgebung des Punktes $c_{t}$ approximiert werden. Weil $c_{t}$ zum Zeitpunkt $t$ schon bekannt ist, muß der Erwartungsoperator nur auf Ausdrücke angewendet werden, die den Konsum der folgenden Periode $t+1$ enthalten.

9 Auch zukünftige Präferenzen können unsicher sein. Überlegungen in diese Richtung sind bisher wenig formalisiert, deswegen gehe ich in dieser Arbeit grundsätzlich von Präferenzen aus, die mit Sicherheit bekannten sind. 


$$
E_{t}\left[\frac{\partial u\left(c_{t+1}\right)}{\partial c_{t+1}}\right] \approx \frac{\partial u\left(c_{t}\right)}{\partial c_{t}}+\frac{\partial^{2} u\left(c_{t}\right)}{\partial c_{t}^{2}} E_{t}\left[c_{t+1}-c_{t}\right]+\frac{1}{2} \frac{\partial^{3} u\left(c_{t}\right)}{\partial c_{t}^{3}} E_{t}\left[\left(c_{t+1}-c_{t}\right)^{2}\right]
$$

Diese Approximation kann in die Eulergleichung (2.9) eingesetzt werden und mit $q=-u^{\prime \prime} / u^{\prime}$, dem Arrow-Pratt MaB der absoluten Risikoaversion und verwandten $\phi=-u^{\prime \prime \prime} / u^{\prime \prime}$, dem Maß der absoluten Vorsichtsneigung ${ }^{10}$, ergibt sich nach einigen einfachen Umformungen die Gleichung:

$$
E_{t}\left[c_{t+1}-c_{t}\right] \approx\left(1-\frac{1+\rho}{1+r}\right) \frac{1}{q\left(c_{t}\right)}+\frac{1}{2} \phi\left(c_{t}\right) E_{t}\left[\left(c_{t+1}-c_{t}\right)^{2}\right]
$$

Die wesentliche Veränderung gegenüber den Modellen unter Sicherheit ist das Moment zweiter Ordnung auf der rechten Seite der Gleichung. Wenn das Einkommen die einzige Quelle der Unsicherheit ist, dann hängt dieser Term eng mit der Varianz des zukünftigen Einkommens zusammen. Die Interpretation sagt, je größer die Unsicherheit über den Verlauf des zukünftigen Einkommens, desto weniger konsumieren die Haushalte am Anfang ihres Lebenszyklusses. Die Haushalte verhalten sich bei Unsicherheit vorsichtig, daher wird diese Klasse von Modellen im Englischen precautionary saving models genannt. Das relative Gewicht des Vorsichtsmotivs wird durch $\phi$, dem Maß der absoluten Vorsichtsneigung, gemessen. Eine hohe Vorsichtsneigung erhöht ceteris paribus die Ersparnisse zu Beginn des Lebens. Im Vergleich zu Modellen unter Sicherheit ist der Konsum bei Unsicherheit am Anfang des Lebenszyklusses geringer und über den Lebenszyklus weniger geglättet.

\section{Empirische Evidenz}

Die quantitative Untersuchung des Vorsichtssparens wurde durch eine Arbeit von Skinner (1988) wesentlich beeinflußt. In seinem Modell besteht Unsicherheit über das Arbeitseinkommen und die Zinssätze. Skinner kann zeigen, daß die Haushalte höchstens die sichere Komponente ihres zukünftigen Einkommens beleihen, aber keinesfalls den unsicheren Teil angreifen. Insgesamt schätzt Skinner den Anteil des Vorsichtssparens auf $50 \%$ der gesamten Lebenszyklusersparnisse. Die große Bedeutung des Vorsichtssparens wird von Caballero (1991) bestätigt, denn nach

10 Kimball (1990) diskutiert ausführlich den Zusammenhang zwischen der auf Arrow (1974) und Pratt (1964) zurückgehenden Theorie der Risikoaversion und der sogenannten Theory of Prudence. Dieser Zusammenhang wird schon durch den Vergleich der Titel deutlich: Der Aufsatz von Pratt heißt "Risk Aversion in the Small and in the Large" und Kimball titelt "Precautionary Saving in the Small and in the Large". 
seiner Analyse können sogar über $60 \%$ des aggregierten Vermögens der USA auf die Einkommensunsicherheit zurückgeführt werden. Weitere Evidenz für das Vorsichtsmotiv bringt Carroll (1994), der verschiedene Maße für die Unsicherheit des Einkommens konstruiert und zeigt, daß sie einen hoch signifikanten Einfluß auf den Konsum haben. Die Zunahme der Unsicherheit um eine Standardabweichung führt in Carolls Schätzungen zu einem Rückgang des Konsums um $3 \%$ bis $5 \%$.

Diese Ergebnisse sprechen eindeutig für Vorsicht als Motiv des Sparens - aber es gibt auch Gegenargumente. Wenn das Vorsichtssparen von Bedeutung ist, dann sollten ceteris paribus die Sparquoten in Ländern mit umfassenden sozialen Sicherunssystemen geringer sein, denn die Hauptaufgabe der Sozialversicherung ist gerade die Absicherung gegen unvorhergesehene Wechselfälle des Lebens. Der internationale Vergleich ergibt aber das Gegenteil: Die Länder mit den weitreichendsten Sozialversicherungen haben die höchsten Sparquoten, wie der Sammelband von Poterba (1994) belegt.

\section{Kritische Würdigung}

So eindeutig die zitierten mirkoökonometrischen Arbeiten den Einfluß von Unsicherheit nachzuweisen scheinen, so deutlich muß man auch deren Grenzen sehen. Caballero (1991) benennt das Hauptproblem: Das Maß der Unsicherheit, das der Ökonometriker konstruieren kann, muß keinesfalls mit der subjektiven Unsicherheit des beobachteten Haushalts übereinstimmen. Für die Entscheidung des Haushalts ist aber gerade diese subjektive ex ante Einschätzung der Unsicherheit maßgebend, der Ökonometriker erhebt die Daten aber gewöhnlich ex post. Welche Bedeutung hat diese Tatsache für die Simulation des allgemeinen Gleichgewichts? Weil die Entwicklung der unsicheren Variablen genau spezifiziert werden muß (beispielsweise durch einen stochastischen Prozeß), die gewählte Spezifikation aber kaum zu überprüfen ist, bleibt offen, wie gut die gewählte Spezifikation die maßgebliche subjektive Einschätzung der Haushalte annähert.

Wenn das Problem der subjektiven Unsicherheit gelöst wäre, ergäbe sich ein weiteres, numerisches Problem: Um den optimalen Konsumverlauf zu bestimmen, muß die Stochastik aus der Optimalitätsbedingung ausintegriert werden. Dazu wird der Zustandsraum gewöhnlich diskretisiert, zum Beispiel muß in dem oben besprochenen Modell mit unsicherem Einkommen die Verteilung des Arbeitseinkommens diskretisiert werden. Also stellt sich auch hier die Frage nach der Güte der Approximation. 


\subsubsection{Andere Erweiterungen}

Intertemporale Separabilität

Der Lebensnutzen wird in Modellen intertemporaler Allokation meistens additivseparabel aus den Periodennutzen zusammengesetzt, denn das vereinfacht die Optimierung ganz wesentlich. Diese Vereinfachung wird allerdings durch starke Restriktionen erkauft: Betrachtet man die marginale Rate der Substitution zwischen zwei Perioden, so ist diese bei additiv-separablem Lebensnutzen unabhängig vom Konsum in anderen Perioden - es gibt lediglich einen indirekten Einfluß über das Lebenseinkommen. Tatsächlich spricht aber vieles dafür, daß der Konsum auch durch Gewohnheiten bestimmt wird, für die in additiv-separablen Spezifikationen kein Platz ist. In einem interessanten Papier hebt Browning (1991) die Separabilitätsannahme auf, indem er ein Konsumgut in aufeinanderfolgenden Perioden entweder als Komplement oder als Substitut zu sich selbst betrachtet. Die empirische Überprüfung des Modells mit Daten aus Großbritannien verwirft signifikant additiv-separable Präferenzen, die als Spezialfall in Brownings Modell enthalten sind. Daten aus Großbritannien verwenden auch Blundell et al. (1994), die ein Nachfragesystem in den intertemporalen Zusammenhang des Lebenszyklusmodells integrieren. Sie zerlegen den Allokationsproze $B$ in die Allokation innerhalb der Perioden - das Nachfragesystem - und die intertemporale Allokation über die Perioden hinweg. Für die intertemporale Allokation gehen Blundell et al. weiter von additiver Separabilität aus, das Nachfragesystem der intratemporalen Allokation schränkt die Substitutionsbeziehungen sehr viel weniger ein. Einen anderen Weg beschreitet Obstfeld (1990) in einer theoretischen Arbeit, in der die Zeitpräferenzrate vom vergangenen Konsum abhängig ist. Damit wird ein Zusammenhang zwischen dem schon realisierten Konsum und dem marginalen Nutzen des gegenwärtigen Konsums hergestellt, so daß Gewohnheiten berücksichtigt werden können ${ }^{11}$.

\section{Zeitpräferenz}

Die intertemporale Separabilität ist eng mit der Zeitpräferenzrate verknüpft, wie ein Blick auf die Lebensnutzenfunktion (2.1) sofort verdeutlicht. Strotz (1956) hat gezeigt, daß nur die in der Lebensnutzenfunktion (2.1) angenommene exponentielle Diskontierung zu einer dynamisch konsistenten Lösung führt. Dynamische Konsistenz der Lösung bedeutet, daß eine erneute Optimierung zu einem späteren Zeitpunkt ceteris paribus exakt das gleiche Ergebnis ergibt wie zum ursprünglichen Zeitpunkt. Eine einzige Optimierung zu Beginn des Lebens reicht also aus,

11 In der englischen Literatur werden solche Modelle als habit formation models bezeichnet. 
um das optimale Konsumprofil für das ganze Leben zu bestimmen. Die aktuelle Forschung zieht die exponentielle Diskontierung zunehmend in Zweifel und schlägt statt dessen "preskriptive Zeitpräferenztheorien“ vor, siehe Loewenstein und Thaler (1989), Ainslie und Haslam (1992), Ahlbrecht und Weber (1995), sowie Laibson (1995). Preskriptive Zeitpräferenztheorien schwächen eine wesentliche Restriktion der exponentiellen Diskontierung ab, weil das Gewicht beim Vergleich aufeinanderfolgender Perioden in der Zukunft abnehmen kann, dagegen beträgt es bei der exponentiellen Diskontierung immer $(1+\rho)$.

Weil die additiv-separablen Präferenzen eng mit der Diskontierung verknüpft sind, besteht für die Zeitpräferenztheorien ein grundsätzliches Problem: Wenn die Separabilitätsannahme die starke Restriktion ist, dann kann eine neue Zeitpräferenztheorie nur wenig ausrichten, denn die Separabilität als wirklich einschneidende Restriktion bleibt bestehen.

\subsubsection{Kritische Würdigung}

Die Erforschung des Sparverhaltens der Haushalte hat dem klassischen Lebenszyklusmodell in den letzten Jahren viele interessante Erweiterungen hinzugefügt und dabei eine Fülle wichtiger Aspekte untersucht. Beispiele sind das Erbschaftsmotiv, Liquiditätsrestriktionen, Sparen aus Vorsichtsmotiven, die Separabilität sowie vielfältige Kombinationen der einzelnen Motive. Gegenüber der einfachsten Version des klassischen Lebenszyklusmodells können diese Erweiterungen eine ungleich feinere Erklärung des Sparverhaltens der Haushalte im Rahmen der neoklassischen Theorie liefern.

Dennoch bleiben grundsätzliche Fragen offen: Wie stark wird das Konsumverhalten der Haushalte durch Gewohnheiten oder Faustregeln bestimmt? Ist die Annahme nutzenmaximierender Haushalte realistisch? Sind die Haushalte überhaupt zur Lösung intertemporaler Optimierungsaufgaben fähig? Ist es richtig, Gewinne und Verluste symmetrisch zu bewerten? Wegen diesen Überlegungen bezweifeln manche Ökonomen, ob der neoklassische Ansatz das menschliche Verhalten überhaupt geeignet beschreiben kann. Ich finde diese Frage sehr wichtig, auch wenn ich den neoklassischen Ansatz prinzipiell für sehr leistungsfähig halte und meine Simulation auf ein neoklassisches Modell gründe. Um die Möglichkeiten, aber auch die Grenzen der die Neoklassik verlassenden Behavioural Economics kennen zu lernen, versuche ich im nächsten Abschnitt, das Sparverhalten der Haushalte mit Elementen aus den Behavioural Economics zu beschreiben. 


\subsection{Mental Accounting und Sustainable Consumption}

Behavioural Economics als Forschungsrichtung ist noch wenig etabliert und schwer abzugrenzen - es existiert noch kein Behavioural Paradigma, es ist noch viel Platz für Experimente. In diesem Abschnitt versuche ich bewußt unbefangen, aus Elementen der Behavioural Economics ein numerisch handhabbares Modell zu entwickeln. Ziel ist es, das Sparverhalten der Haushalte aus einem ungewohnten Blickwinkel zu betrachten, um so neue Einsichten zu bekommen.

\subsubsection{Mental Accounting}

Für neoklassische Modelle des Sparverhaltens sind eine Reihe von Annahmen typisch, die ich hier noch einmal übersichtlich zusammenfasse:

- Die Haushalte sind rationale, vorausschauende Nutzenmaximierer.

- Die Haushalte können die intertemporale Optimierungsaufgabe ohne große Kosten lösen.

- Die Vergangenheit hat allenfalls über die Budgetrestriktion einen Einfluß auf zukünftige Entscheidungen, von Gewohnheiten wird meistens abstrahiert.

- Gewinne und Verluste werden symmetrisch bewertet.

- Die Bewertung eines Gutes hängt bei gleichem Einkommen nicht davon ab, ob man es besitzt oder nicht.

Eine weitere Annahme ist die sogenannte Fungibilitätsannahme:

- Verschiedene finanzielle Assets, Immobilien und sonstiges Vermögen werden als vollständige Substitute betrachtet.

Die Fungibilitätsannahme ist der Ausgangspunkt für das von Shefrin und Thaler (1988) in die Diskussion gebrachte Mental Accounting Modell, denn das Mental Accounting Modell hebt die Fungibilitätsannahme auf. Shefrin und Thaler kritisieren, da $\beta$ in Lebenszyklusmodellen alle Vermögenstitel perfekte Substitute sind und daher kein Einfluß von der Zusammensetzung des Vermögens auf die Konsumentscheidungen der Haushalte ausgeht. Ein einfaches Beispiel macht deutlich, welche Konsequenzen sich aus der Fungibilitätsannahme für das Verhalten der Haushalte ergeben: Die Konsumänderungen von einem Haushalt, der eine Immobilie im Marktwert von 100.000,- DM erbt und einem Haushalt, der 100.000,DM Bargeld erbt, sind ceteris paribus gleich. Ein Maß für diese Konsumänderung ist die marginale Konsumneigung, die angibt, wie eine zusätzliche, marginale Vermögenseinheit den Konsum beeinflußt. Die marginale Konsumneigung liegt grundsätzlich zwischen null und eins; wenn sie null ist, dann hat eine zusätzliche Vermögenseinheit keine Auswirkungen auf den Konsum; wenn sie eins ist, dann 
wird eine zusätzliche Vermögenseinheit vollständig konsumiert. In dem Beispiel mit den Erbschaften ist die marginale Konsumneigung unabhängig von der Art des geerbten Vermögens, tatsächlich dürfte sie aber für das Bargeld erheblich höher sein als für die Immobilie.

Shefrin und Thaler heben in ihrem Modell die Fungibilitätsannahme auf, indem sie das Vermögensaggregat durch ein System von Mental Accounts ersetzen, so da $B$ die Haushalte verschiedene Assets verschiedenen Konten zuordnen können. $\mathrm{Ob}$ diese Konten bei einer Bank tatsächlich existieren oder ob diese Konten nur im Kopf der Leute geführt werden (daher mental accounting), ist unerheblich. Das Modell von Shefrin und Thaler geht von drei Mental Accounts aus: Laufendes Einkommen wird dem Konto I (current income) zugeordnet, Konto $F$ (future income) enthält zukünftiges Einkommen und das restliche Vermögen befindet sich im Assetkonto A. Drei Mental Accounts decken natürlich nicht die Vielfalt der unterschiedlichen Vermögensarten ab, gegenüber dem einen Konto in Modellen voller Fungibilität werden die Restriktionen aber doch gelockert.

\section{Mental Accounting Nutzenfunktion}

Wenn sich die Mental Accounts nur durch unterschiedliche Transaktionskosten unterscheiden würden, dann könnte mit den Mental Accounts ein neoklassisches Modell entwickelt werden. Die Motivation wäre dann, daß Geldentnahmen vom Girokonto (current income I) kaum Transaktionskosten zur Folge haben, während der Verkauf von Anleihen oder Aktien einen höheren Aufwand verursacht und die Beleihung des zukünftigen Einkommens noch teurer ist. Aber Shefrin und Thaler beschreiten einen anderen Weg und stützen sich auf psychologische Untersuchungen, die Thaler wie folgt zusammenfaßt:

"Households [...] can be thought of as following the following prudent rule:

[...] Live within your means. Do not borrow from $F$ or $A$ to increase current consumption, except during well-defined emergencies." (Thaler, 1990, p. 195)

Wenn sich die Haushalte nach dieser Regel verhalten, werden sie normalerweise weder die Assets noch das zukünftige Einkommen (das auch mit Humankapital bezeichnet wird) zur Finanzierung ihres laufenden Konsums verwenden. Das Argument ist grundverschieden von der neoklassischen Transaktionskosten-Überlegung, denn es zielt auf die Präferenzen und nicht auf die Restriktionen der Haushalte. Folgerichtig versuchen Shefrin und Thaler, die zitierte Verhaltensregel in die Nutzenfunktion zu integrieren. Dazu nehmen sie an, daß ein Haushalt zumindest seinen Willen anstrengen muß, um die Verhaltensregel $\mathrm{zu}$ brechen - und diese Anstrengung reduziert den Nutzen. 
Mit den von Shefrin und Thaler vorgeschlagenen Mental Accounts I (laufendes Einkommen), $A$ (Assets) und $F$ (zukünftiges Einkommen) zerfällt die Konsumnutzenfunktion in drei Bereiche, denn sobald sich der Haushalt über die Verhaltensregel hinwegsetzt und Teile seines Vermögens, das in den Mental Accounts $A$ und $F$ verbucht ist, zur Finanzierung des laufenden Konsums verwendet, wird sein Nutzen durch die Willensanstrengung reduziert. Abbildung 2.2 unterstellt, $\mathrm{da} ß$ der Haushalt erst nach der Auflösung seines sonstigen Vermögens $A$ auf sein Humankapital $F$ zurückgreift. Die der Abbildung entsprechende Parametrisierung der Nutzenfunktion durch die Mental Accounts ist einfach anzugeben:

$$
\tilde{u}(c \mid l, A, F)= \begin{cases}u(c) & , \text { falls } 0<c \leq l \\ u(c)-\Pi^{A} & , \text { falls } l<c \leq A \\ u(c)-\Pi^{A}-\Pi^{F}, & \text { falls } A<c \leq F\end{cases}
$$

Die Parameter $\Pi^{A}$ und $\Pi^{F}$ sind positiv und stehen für die Nutzenabschläge, die durch die Willensanstrengung beim Übertreten der Verhaltensregel entstehen. Die Nutzenfunktion (2.12) unterstellt, daß die Nutzenabschläge erstens unabhängig von der Höhe des abgehobenen Betrags sind und zweitens nicht von den Beträgen auf den Mental Accounts abhängen.

Abbildung 2.2: Konsumnutzen im Mental Accounting Modell

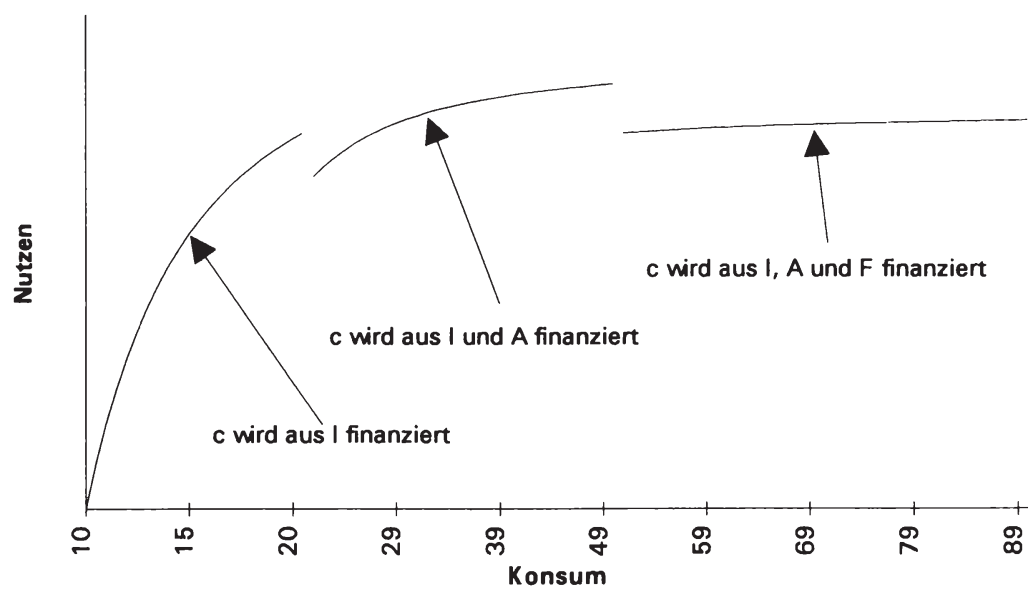

Quelle: Eigene Darstellung nach Shefrin und Thaler (1988) 
Die Sprungstellen, die durch die Mental Accounts bestimmt werden, ergeben für die jeweiligen Mental Accounts unterschiedliche marginale Konsumneigungen: Eine zusätzliche Einheit Humankapital hat einen geringen Einfluß auf den Konsum, zusätzliche Assets werden schon eher zur Finanzierung des Konsums verwendet, und die höchste marginale Konsumneigung ergibt sich für das laufende Einkommen.

Die „marginale Konsumneigung“ ist von dem „marginalen Konsumnutzen“ zu unterscheiden. Letzterer quantifiziert die Auswirkung, die eine marginale Änderung des Konsums auf den Nutzen hat und wird daher auch „Grenznutzen des Konsums“ genannt. Der marginale Konsumnutzen der Nutzenfunktion (2.12) führt zu folgender Beobachtung: Abbildung 2.2 zeigt, daß die ersten marginalen Konsumeinheiten, die aus dem Asset- oder zukünftigem Einkommenskonto finanziert werden, extrem teuer sind. Der zusätzliche Nutzen dieser „ersten“ Konsumeinheiten ist geringer als der Nutzenabschlag, also lohnt es sich nicht, die Vermögenskonten für kleine Beträge anzugreifen.

Eine weitere Aussage ergibt sich aus der Beziehung zwischen Nutzenabschlägen und marginalem Konsumnutzen. Weil der marginale Konsumnutzen von der Höhe des Konsums abhängt, die Nutzenabschläge aber exogen fixiert sind, weichen die beiden Größen mit zunehmendem Konsum immer weiter voneinander ab. Haushalte mit niedrigem Einkommen haben ein niedriges Konsumniveau aber einen hohen marginalen Konsumnutzen, so daß sie schon bei sehr viel kleineren Beträgen die Vermögenskonten angreifen. Anders ist die Situation für Haushalte mit hohem Einkommen, denn der marginale Konsumnutzen ist relativ zu den Nutzenabschlägen gering. Folglich wird das Vermögen allenfalls zur Finanzierung großer Konsumausgaben ${ }^{12}$ reduziert, aber keinesfalls wegen kleiner Beträge.

Einige Beispiele sollen die bisherigen Ergebnisse aus der theoretischen Erörterung des Mental Accounting Modells verdeutlichen. Im Gegensatz zum klassischen Lebenszyklusmodell kann das Mental Accounting Modell erklären, warum eine empfangene Erbschaft einen geringeren Einfluß auf den Konsum hat als eine dauerhafte Lohnerhöhung. Der Lohnanstieg erhöht das laufende Einkommenskonto $I$, dessen marginale Konsumneigung am höchsten ist. Die Erbschaft erhöht aber das Assetkonto $A$, dessen marginale Konsumneigung geringer ist als die marginale Konsumneigung von $I$.

Weil die Nutzenabschläge in der hier vorgestellten Parametrisierung des Mental Accounting Modells konstant sind, der marginale Konsumnutzen bei steigenden

12 Hier stellt sich die Frage, ob große Konsumausgaben, die typischerweise beim Kauf von langlebigen Konsumgütern entstehen, zumindest teilweise dem Vermögen zuzurechnen sind (siehe Abschnitt 2.1.2). 
Konsumausgaben aber abnimmt, ist Entsparen besonders für Haushalte mit höherem Einkommen teuer. Daher haben Haushalte mit glatten Einkommensprofilen im Mental Accounting Modell ceteris paribus einen höheren Lebensnutzen, denn glatte Einkommensprofile ermöglichen Konsumglättung ohne Entsparen. Je nach Definition der Mental Accounts sind Renten- und Lebensversicherungen eine Möglichkeit, um das Einkommensprofil zu glätten. Wenn diese Versicherungen bis zu ihrer Fälligkeit nicht gekündigt und beliehen werden können, dann zählen sie nicht zum Assetkonto des Haushalts. Die späteren Auszahlungen der Rentenund Lebensversicherungen erhöhen das laufende Einkommenskonto $I$, ohne die Verhaltensregel der Haushalte zu brechen, weil Renten- und Lebensversicherungen Sparformen sind, die ohne Nutzenabschläge aufgelöst werden können. Erkauft wird dieser Vorteil durch eingeschränkte Entscheidungsmöglichkeiten, denn wenn das Geld in die Renten- und Lebensversicherung eingezahlt ist, können die Haushalte nicht mehr frei darüber verfügen. ${ }^{13}$

\section{Überleitung zu Sustainable Consumption}

Die theoretische Betrachtung des Mental Accounting Modells bringt erste interessante Ergebnisse, aber noch keine Konsum- und Vermögensprofile. Um diese zu erhalten, muß die Mental Accounting Nutzenfunktion (2.12) in einen intertemporalen Kontext gestellt werden. In der Theorie ist das einfach: Die Mental Accounting Nutzenfunktion ersetzt in der Lebensnutzenfunktion (2.1) den gewöhnlichen Konsumnutzen. In der numerischen Umsetzung wird es schwieriger: Die Mental Accounting Nutzenfunktion ist nicht stetig und verletzt daher eine wesentliche Voraussetzung effizienter Optimierungsverfahren. Zwar stehen mit dem Downhill Simplex Algorithmus von Nelder und Mead (1965) und dem Simulated Annealing Verfahren von Kirkpatrick et al. (1983) zwei heuristische Methoden zur Lösung mehrdimensionaler, nicht stetiger Optimierungsaufgaben zur Verfügung, aber wenn die Dimensionen des Lösungsraums zunehmen, geraten beide Verfahren schnell an ihre Grenzen. Also habe ich mich nach einer anderen Möglichkeit umgesehen.

13 Diese Einschränkung der Entscheidungsmöglichkeiten interpretieren Shefrin und Thaler (1992) sowie Laibson (1994) als Mittel der Selbstkontrolle, mit dem die Haushalte sich vor den Versuchungen kurzfristig interessanter, aber langfristig ungünstigen Entscheidungen schützen. 


\subsubsection{Sustainable Consumption}

Sustainable Consumption könnte mit dem Konsum umschrieben werden, der langfristig aufrechtzuerhalten ist. Ich bleibe aber bei der von Pemberton (1993) eingeführten englischen Bezeichnung. Pemberton verwendet Sustainable Consumption zur Lösung stochastischer Lebenszyklusmodelle. Die dabei auftretenden Probleme sind sehr ähnlich zu den gerade geschilderten Schwierigkeiten bei der Lösung des Mental Accounting Modells.

Der Grundgedanke des Sustainable Consumption Konzepts ist einfach: Die Haushalte entscheiden über den gegenwärtigen und den zukünftigen Konsum, ohne dabei den zukünftigen Konsum auf einzelne Perioden aufzuteilen. Dadurch wird das n-dimensionale Optimierungsproblem auf die optimale Wahl von zwei Größen reduziert - den gegenwärtigen Konsum und den zukünftigen "durchschnittlichen“ Konsum, der Sustainable Consumption genannt wird. Also ist Sustainable Consumption das konstante Konsumniveau, das die vorhandenen Ressourcen gerade ausschöpft. Die intertemporale Budgetrestriktion im Zeitpunkt $\tau$ enthält nun nicht mehr $T-\tau$ verschiedene Konsumvariablen, statt dessen werden die zukünftigen Konsumvariablen durch Sustainable Consumption $c_{\tau}^{*}$ ersetzt:

$$
c_{\tau}+\sum_{t=\tau+1}^{T}(1+r)^{\tau-t} c_{\tau}^{*}=(1+r) a_{\tau}+\sum_{t=\tau}^{T}(1+r)^{\tau-t} w_{t}
$$

Die Budgetrestriktion (2.13) kann nach $c_{\tau}{ }^{*}$ aufgelöst werden, so daß $c_{\tau}{ }^{*}$ als Funktion von $c_{\tau}$ erscheint:

$$
c_{\tau}^{*}\left(c_{\tau}\right)=\frac{r}{1-(1+r)^{\tau-\tau}}\left[(1+r) a_{\tau}-c_{\tau}+\sum_{t=\tau}^{T}(1+r)^{\tau-t} w_{t}\right]
$$

Solange das Einkommen exogen ist, wird durch die Wahl von $c_{\tau}$ gleichzeitig $c_{\tau}{ }^{*}$ bestimmt, wodurch sich die intertemporale Optimierungsaufgabe entscheidend vereinfacht. Wenn die zukünftigen Konsumvariablen in der Lebensnutzenfunktion (2.1) durch Sustainable Consumption ersetzt werden, ergibt sich eine Lebensnutzenfunktion, die nur noch von $c_{\tau}$ und $c_{\tau}{ }^{*}$ abhängt.

$$
\begin{aligned}
U\left(c_{\tau}\right) & =u\left(c_{\tau}\right)+\sum_{t=\tau+1}^{T}(1+\rho)^{\tau-t} u\left(c_{\tau}^{*}\left(c_{\tau}\right)\right) \\
& =u\left(c_{\tau}\right)+\frac{1-(1+\rho)^{\tau-\tau}}{\rho} u\left(c_{\tau}^{*}\left(c_{\tau}\right)\right)
\end{aligned}
$$


Weil $c_{\tau}{ }^{*}$ nach Gleichung (2.14) eine Funktion von $c_{\tau}$ ist, enthält die Lebensnutzenfunktion (2.15) als einzige endogene Variable den gegenwärtigen Konsum, dessen optimaler Wert durch Differenzieren von (2.15) festgelegt wird:

$$
u^{\prime}\left(c_{\tau}\right)=\frac{r}{\rho} \frac{1-(1+\rho)^{\tau-T}}{1-(1+r)^{\tau-T}} u^{\prime}\left(c_{\tau}{ }^{*}\left(c_{\tau}\right)\right)
$$

Auf den ersten Blick hat die Optimalitätsbedingung (2.16) Ähnlichkeit mit der Eulergleichung (2.3) des klassischen Lebenszyklusmodells. Auf den zweiten Blick wird aber ein fundamentaler Unterschied deutlich: Gleichung (2.16) hebt die intertemporale Verknüpfung der Konsumvariablen auf und ist deshalb keine Eulergleichung. Trotzdem ist das Sustainable Consumption Modell nicht statisch, denn über die Sustainable Consumption Variable wird die Zukunft sehr wohl berücksichtigt. Der marginale Nutzen aus Sustainable Consumption (dem durchschnittlichen zukünftigen Konsumniveau) muß in Relation zum marginalen Nutzen des gegenwärtigen Konsums stehen. Wenn Zeitpräferenzrate und Zinssatz gleich sind, müssen gegenwärtiger Konsum und Sustainable Consumption den gleichen marginalen Nutzen haben und sind folglich identisch. Aus dieser Tatsache kann aber nicht allgemein geschlossen werden, daß bei $\rho=r$ ein flaches Konsumprofil realisiert wird. Weil die intertemporale Verknüpfung in der Optimalitätsbedingung (2.16) nur indirekt über Sustainable Consumption vermittelt wird, bestimmt das Optimum in Periode $t$ nicht gleichzeitig die Optima der restlichen Perioden; vielmehr wird in der nächsten Periode $t+1$ ein neues Optimum bestimmt, welches keineswegs mit dem in der Vorperiode geplanten Sustainable Consumption Niveau übereinstimmen muß. Im klassischen Lebenszyklusmodell beschreibt die Eulergleichung das Konsumprofil über den ganzen Lebenszyklus im Sustainable Consumption Modell ergibt sich aus der Optimalitätsbedingung (2.16) nur der optimale Konsum einer Periode. Um das Konsumprofil, das die Lebensnutzenfunktion (2.15) maximiert, zu bestimmen, muß die Optimalitätsbedingung (2.16) in jeder Periode neu berechnet werden. Also ist das Sustainable Consumption Modell nicht zeitkonsistent. Das ist der zentrale Unterschied zwischen dem Sustainable Consumption Modell und dem klassischen Lebenszyklusmodell, denn im Sustainable Consumption Modell wird die Zeitkonsistenz von vornherein aufgegeben. Die Leute haben im Sustainable Consumption Modell nicht die Absicht, am Lebensanfang einen optimalen Plan aufzustellen um ihn dann bis zum Lebensende zu befolgen - insofern ist die Zeitkonsistenz kein sinnvolles Kriterium zur Beurteilung des Sustainable Consumption Modells. Die Überlegung im Sustainable Consumption Modell ist anders: Das Entscheidungsproblem wird vereinfacht, indem in jeder Periode $t$ nur der gegenwärtige Konsum $c_{t}$ und Sustainable Consumption $c_{\tau}{ }_{\tau}$ betrachtet wird. Der gegenwärtige Konsum wird realisiert, aber Sustainable Consumption hat nach der Entscheidung als reine 
Hilfsgröße keine Bedeutung mehr, denn in der nächsten Periode wird das Entscheidungsproblem neu gelöst. Diese in jeder Periode notwendige Neuplanung ist der Preis, mit dem die auf zwei Variablen vereinfachte Entscheidung erkauft wird.

Ein Beispiel soll verdeutlichen, daß Sustainable Consumption trotz Neuplanung in jeder Periode eine große Vereinfachung gegenüber der Optimierungsaufgabe im Lebenszyklusmodell ist. Wenn der Konsum keine stetige Größe wäre sondern nur $n$ verschiedene Werte annehmen könnte, dann wäre das optimale Konsumprofil des klassischen Lebenszyklusmodells bei einem Lebenszyklus von $T>2$ Perioden aus der Menge der $n^{T}$ möglichen Konsumprofile auszuwählen. Im Sustainable Consumption Modell wird die Auswahlmenge ganz erheblich reduziert: Sie enthält nur noch $n^{2}(T-1)$ Möglichkeiten.

\section{Güte der Approximation}

Bisher ist nicht geklärt, wie weit das Profil des realisierten Konsums im Sustainable Consumption Modell vom Konsumprofil des klassischen Lebenszyklusmodells abweicht. Das realisierte Konsumprofil im Sustainable Consumption Modell ergibt sich aus einer ex post Betrachtung, denn im Sustainable Consumption Modell plant eine Person in jedem Lebensjahr neu. Wenn Zinssatz und Zeitpräferenzrate gleich sind, dann sind die Konsumprofile der beiden Modelle in der ex post Betrachtung gleich, wie Anhang A1 beweist. Wenn sich Zeitpräferenzrate und Zinssatz unterscheiden, weicht das realisierte Konsumprofil im Sustainable Consumption Modell im allgemeinen vom Konsumprofil des Lebenszyklusmodells ab. Tabelle 2.1 versucht, die Abweichung der beiden Profile abzuschätzen. Variiert werden der Zinssatz und die Zeitpräferenzrate, denn wenn $\rho=r$ gilt, werden in beiden Modellen die gleichen Konsumprofile realisiert. Das Arbeitseinkommen ${ }^{14}$, die Nutzenfunktion $u(c)=c^{(1-\gamma)} /(1-\gamma)$ mit $\gamma=3$ und das Anfangsvermögen $a_{0}=0$ bleiben jeweils unverändert. Die drei Maße $M_{1}, M_{2}$ und $M_{3}$ messen die Nutzenabweichung mit unterschiedlichen Konzepten und sind wie folgt definiert:

1. Maß 1 mißt die prozentuale Abweichung zwischen dem Lebensnutzen aus dem optimalen Konsumprofil $C^{L}$ des klassischen Lebenszyklusmodells und dem Lebensnutzen aus dem realisierten Konsumprofil $c^{s}$ des Sustainable Consumption Modells:

14 Das Einkommensprofil ist durch ein Polynom zweiten Grades gegeben, daß im Jahr des Arbeitsanfangs den Wert 1 annimmt und mit dem Jahr der Verrentung sein Maximum mit dem doppelten des Anfangseinkommens erreicht. Nach der Verrentung wird eine Rente in Höhe von $70 \%$ des durchschnittlichen Arbeitseinkommens gezahlt. 


$$
M_{1}=\left|\frac{U\left(\vec{c}^{L}\right)-U\left(\vec{c}^{s}\right)}{U\left(\vec{c}^{L}\right)}\right| \cdot 100
$$

2. $\mathrm{Ma} 22$ versucht, die Nutzendifferenz durch eine in Vermögenseinheiten interpretierbare Größe auszudrücken und beruht auf der folgenden Überlegung: Um welchen Betrag $M_{2}$ muß das Anfangsvermögen im Sustainable Consumption Modell erhöht werden, damit der Lebensnutzen im Sustainable Consumption Modell und im klassischen Lebenszyklusmodell gleich sind? Die entsprechende formale Schreibweise definiert $M_{2}$ als den Kompensationsbetrag, der die folgende Gleichung erfüllt:

$$
U\left(\vec{c}^{L} \mid a_{1}, \vec{w}, \vec{r}, \rho\right)=U\left(\vec{c}^{S} \mid a_{1}+M_{2}, \vec{w}, \vec{r}, \rho\right)
$$

Weil das Vermögen in Geldeinheiten gemessen wird, hängt Maß 2 von diesen Einheiten $\mathrm{ab}$.

3. Maß 3 hängt eng mit Maß 2 zusammen, denn es berechnet den Kompensationsbetrag $M_{2}$ als Prozent des auf die Anfangsperiode abdiskontierten Lebens-Arbeitseinkommens, ist im Unterschied zu Maß 2 allerdings unabhängig von Geldeinheiten.

$$
M_{3}=\frac{M_{2}}{\sum_{t=\tau}^{T}\left(1+r_{t}\right)^{\tau-t} W_{t}} \cdot 100
$$

Tabelle 2.1: Abweichung des Sustainable Consumption Optimums vom Optimum des klassischen Lebenszyklusmodells

\begin{tabular}{|r|r|r|r|r|}
\hline$r$ & $\rho$ & $M_{1}$ & $M_{2}$ & $M_{3}$ \\
\hline 2,0 & 5,0 & 0,10 & 0,03 & 0,06 \\
\hline 5,0 & 2,0 & 0,10 & 0,01 & 0,05 \\
\hline 2,0 & 10,0 & 3,02 & 0,84 & 1,76 \\
\hline 10,0 & 2,0 & 2,72 & 0,18 & 1,24 \\
\hline
\end{tabular}

Quelle: Eigene Berechnungen 
Die Ergebnisse in Tabelle 2.1 zeigen für alle drei Maße, daß die Abweichung zwischen dem Optimum des klassischen Lebenszyklusmodells und dem Sustainable Consumption Optimum erstens klein ist und zweitens mit steigender Differenz zwischen Zinssatz und Zeitpräferenzrate zunimmt. Die Kompensationsbeträge $M_{2}$ sind in allen Fällen kleiner als das Einstiegsgehalt, das eine Geldeinheit beträgt. Ein interessanter Vergleich ergibt sich, wenn man den prozentualen Anteil des Kompensationsbetrags auf ein realistisches Lebens-Arbeitseinkommen ${ }^{15}$ bezieht. Dieser Vergleich berücksichtigt im Gegensatz zum Maß $M_{2}$ auch den Zinssatz, denn das auf die Anfangsperiode abdiskontierte Lebens-Arbeitseinkommen hängt von den Zinsen ab. Bei einem Gehalt von $10000 \mathrm{DM}$ für das erste Arbeitsjahr, das während des Erwerbslebens bis auf 20000 DM ansteigt, entspricht der Kompensationsbetrag bei einem Zinssatz von $2 \%$ und einer Zeitpräferenzrate von $5 \%$ knapp $271 \mathrm{DM}$. Um diesen Betrag müßte das Anfangsvermögen erhöht werden, damit der Lebensnutzen in beiden Modellen gleich wäre. Wenn die Lösung der Optimierungsaufgabe nach dem klassischen Lebenszyklusmodell höhere Kosten verursacht als die im Sinne des Lebenszyklusmodells approximative Sustainable Consumption Lösung, dann ist die Sustainable Consumption Lösung günstiger, sobald der Kostenunterschied mehr als $271 \mathrm{DM}$ beträgt. Selbst bei einem Zinssatz von $10 \%$ und einer Zeitpräferenzrate von $2 \%$ wäre das Sustainable Consumption Modell bei einem Kostenunterschied von mehr als 1806 DM dem klassischen Lebenszyklusmodell vorzuziehen.

\section{Beispiel}

Abschließend vergleiche ich für einen Beispielhaushalt das Sustainable Consumption Profil mit dem Konsumprofil des klassischen Lebenszyklusmodells. Die Präferenzen des Haushalts sind durch die isoelastische Nutzenfunktion $u(c)=$ $c^{(1-\gamma)} /(1-\gamma)$ mit $\gamma=3$ gegeben. Der Haushalt beginnt im Alter von 21 Jahren zu arbeiten und erhält ein auf $w_{21}=1$ normiertes Einstiegsgehalt. Während des Erwerbslebens steigt das Einkommen mit abnehmender Rate an und erreicht im Alter von 65 Jahren, dem Verrentungsalter, sein Maximum von $w_{65}=2$. Die Rente ist auf $70 \%$ des letzten Einkommens festgelegt. Weil der Unterschied zwischen Zeitpräferenzrate und Zinssatz die Abweichungen zwischen dem realisierten Konsumprofil des Sustainable Consumption Modells und dem Konsumprofil des Lebenszyklusmodells bestimmt, unterscheiden sich die konstante Zeitpräferenzrate von $\rho=5 \%$ und der konstante Zinssatz von $r=2 \%$ deutlich.

15 In der Definition des Maßes $M_{3}$ umfaßt das Lebens-Arbeitseinkommen auch die Rentenzahlungen. 
Die stark durchgezogene Linie in Abbildung 2.3 ist das realisierte Konsumprofil der Sustainable Consumption Optimierung - es unterscheidet sich nur wenig von dem schwach durchgezogenen Konsumprofil des klassischen Lebenszyklusmodells. Am Anfang des Erwerbslebens konsumiert der nach dem Sustainable Consumption Konzept planende Haushalt etwas weniger als ein Haushalt, der das Konsumprofil des klassischen Lebenszyklusmodells realisiert, doch ab dem 41 . Lebensjahr liegt das realisierte Konsumprofil des Sustainable Consumption Modells über dem Vergleichsprofil.

Abbildung 2.3: Sustainable Consumption im Vergleich zum klassischen Lebenszyklusmodell

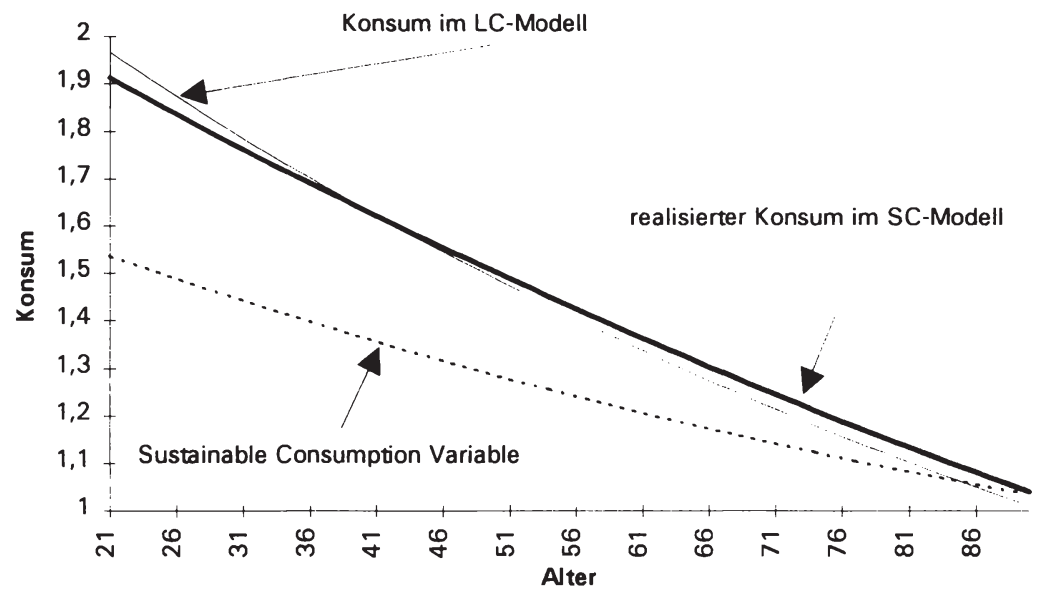

Anmerkungen:LC-Modell steht für das klassische Lebenszyklusmodell SC-Modell steht für das Sustainable Consumption Modell.

Quelle: $\quad$ Eigene Berechnungen

Der Lebensnutzen aus dem Konsumprofil des Sustainable Consumption Modells weicht weniger als $0,1 \%$ vom Lebensnutzen des klassischen Lebenszyklusmodells ab. Für das Verständnis des Sustainable Consumption Modells ist es wichtig, das realisierte Konsumprofil von dem Profil der Sustainable Consumption Variablen zu unterscheiden. Letzteres wird nicht realisiert; es ist eine Hilfsgröße, mit der die Haushalte die zukünftigen Auswirkungen ihrer gegenwärtigen Entscheidungen abschätzen. Das Profil der Sustainable Consumption Variablen liegt in Abbildung 2.3 während des ganzen Lebens unter dem realisierten Konsum- 
profil. Also weiß der Haushalt in jedem Planungszeitpunkt, daß er das realisierte Konsumniveau langfristig nicht halten kann. Die Sustainable Consumption Variable ist folglich eine langfristig vorausschauende Hilfsvariable - sie soll nicht vorhersagen, welcher Konsum in der nächsten Periode realisiert wird. In Abbildung 2.3 wird die langfristig vorausschauende Intention der Sustainable Consumption Variablen besonders deutlich: Schon in der ersten Periode weiß der Haushalt, daß sein Konsum später erheblich fallen muß, denn der Unterschied zwischen dem realisierten Konsum und der Sustainable Consumption Variablen ist sehr groß.

Kritische Würdigung

Von den Vertretern der Behavioural Economics wird die Neoklassische Theorie unter anderem kritisiert, weil sie ihre Ergebnisse mit überaus komplizierten Optimierungsverfahren herleitet. So kritisiert Hey (1983):

"I would ague that in many real-life problems it is simply impossible to carry out the backward induction procedure. Either the problem gets so hideously complex that it is beyond the computational power of the decision-maker; or the sequence of implications stretches so far into the future that the consequences get shrouded in the mists of time." (Hey, 1983, p.137)

In die gleiche Richtung geht die Kritik Pemberton's (1993), der sich auf das Lebenszyklusmodell bei unsicherem Einkommen bezieht und sagt:

"[...] since in the presence of income uncertainty it involves numerical and analytical complexity well beyond the capacity of the human brain (and indeed, apparently well beyond the capacity of available computers)." (Pemberton, 1993, p. 7)

Mit der mathematischen Optimierungstheorie verfehlten die Neoklassiker, so das Argument mancher Behavioural Economists, völlig das tatsächliche Verhalten der Haushalte, denn kaum ein Haushalt beherrsche die mathematische Optimierungstheorie. Daher versucht Behavioural Economics Modelle zu konstruieren, deren Elemente von den Haushalten verstanden und angewendet werden können. Wenn man annimmt, daß Haushalte sich nach der Maxime "Entscheide über die Gegenwart und die Zukunft, aber zerlege die Zukunft nicht in einzelne Perioden" verhalten, dann ist Sustainable Consumption ein Konzept der Behavioural Economics, das nicht irgendeine Approximation an das mathematisch exakte Optimum ist, sondern einem im Sinne der Behavioural Economics interpretierbaren Haushaltsverhalten entspricht. 
Eine andere Interpretation ist neoklassisch: Sustainable Consumption ist ein approximatives Optimierungsverfahren, das eine gute Approximation an die optimale Lösung des klassischen Lebenszyklusmodells liefert. Wenn Optimierungskosten zu berücksichtigen sind, dann dominiert nach Tabelle 2.1 eine mit dem Sustainable Consumption Modell gewonnene Lösung schon bei relativ geringen Kostenvorteilen die Lösung des klassischen Lebenszyklusmodells. Ich bevorzuge diese zweite Interpretation und sehe eine Vielzahl von Anwendungsmöglichkeiten des Sustainable Consumption Modells, zum Beispiel in Modellen mit unsicherem Einkommen. Es wäre sehr interessant, solche Modelle mit dem Sustainable Consumption Konzept zu lösen und die so gewonnen Ergebnisse mit anderen Lösungsverfahren zu vergleichen, zum Beispiel den auf einem Vektorrechner per Diskretisierung des Einkommensraumes abgeleiteten Ergebnissen von Hubbard et al. (1995). In dieser Arbeit habe ich das Sustainable Consumption Modell aber aus einem anderen Grund eingeführt: Ich möchte Konsum- und Vermögensprofile des Mental Accounting Modells simulieren.

\subsubsection{Simulation des Mental Accounting Modells}

Bevor die ersten Konsumprofile des Mental Accounting Modells mit Sustainable Consumption als approximativem Optimierungsverfahren simuliert werden können, sind noch einige Anpassungen nötig. Im Mental Accounting Modell wird die Budgetrestriktion (2.2) durch eine Ungleichheitsbedingung ersetzt, die fordert, $\mathrm{da} ß$ der Konsum in keiner Periode die vorhandenen Ressourcen übersteigen darf:

$$
c_{t} \leq I_{t}+A_{t}+F_{t} \quad \text { für } t=\tau, \ldots, T
$$

Die verfügbaren Ressourcen befinden sich in den drei Mental Accounts $I, A$, und $F$, die nun eindeutig voneinander abgegrenzt werden müssen. Das laufende Einkommenskonto / enthält das Arbeits- und Zinseinkommen einer Periode. Während die Zuordnung des Arbeitseinkommens unstrittig ist, könnte man das Zinseinkommen auch als Teil des Vermögens betrachten. In diesem Fall wäre das Vermögen als Einkommensquelle unattraktiv, weil die Zinsen vom Vermögenskonto abgebucht werden müßten und dann der Nutzenabschlag fällig wäre. Ist das Zinseinkommen aber Teil des laufenden Einkommenskontos, dann kann es (wegen des abnehmenden Grenznutzens des Konsums und des fixen Nutzenabschlags) besonders für Haushalte mit hohem Arbeitseinkommen sinnvoll sein, während des Arbeitslebens ein Vermögen aufzubauen, dessen Zinsen später die 
Rente aufbessern. Aus diesem Grund ${ }^{16}$ ordne ich das Zinseinkommen dem laufenden Einkommenskonto zu und abstrahiere damit von Anlageformen, die Zinsen thesaurieren. Das Assetkonto A enthält dann den Bestand der Assets am Anfang einer Periode.

Bei der Definition des zukünftigen Einkommenskontos $F$ ergeben sich erneut mehrere Möglichkeiten. Nach der Budgetrestriktion (2.17) besteht die Möglichkeit, das zukünftige Einkommen zu beleihen um den gegenwärtigen Konsum zu erhöhen. Das zukünftige Netto-Arbeitseinkommen gehört daher zweifelsfrei zum zukünftigen Einkommenskonto. Die Rente verbuche ich dagegen nicht auf dem zukünftigen Einkommenskonto, denn in den meisten Ländern, so auch in Deutschland, ist die Rente nicht beleihbar. Durch einen definitorischen Trick löse ich das Problem des zukünftigen Zinseinkommens. Schon bei der Vorstellung der von Shefrin und Thaler vorgeschlagenen Nutzenfunktion in Abbildung 2.2 erscheinen die Konten in einer Rangfolge: Das laufende Einkommen / ist mit der höchsten marginalen Konsumneigung assoziiert, dann kommt das Assetkonto $A$ und zuletzt steht das zukünftige Einkommen $F$ mit der geringsten marginalen Konsumneigung. Der Argumentation von Shefrin und Thaler folgend nehme ich an, daß das zukünftige Einkommen erst dann beliehen wird, wenn sowohl das laufende Einkommen als auch die gesamten Assets verbraucht sind - womit das Problem des zukünftigen Zinseinkommens nicht länger existiert. Ich fasse die Definition der Mental Accounts kurz zusammen:

- laufendes Einkommenskonto I: laufendes Arbeits- und Zinseinkommen

- Assetkonto A: Assets

- zukünftiges Einkommenskonto $F$ : zukünftiges Arbeitseinkommen

Mental Accounting und Sustainable Consumption

Mit der Definition der Mental Accounts ist die Optimierungsaufgabe des Mental Accounting Modells nun wohldefiniert: In jeder Periode $j$ sind der gegenwärtige Konsum $c_{j}$ und Sustainable Consumption $c_{j}^{*}$ hinsichtlich der folgenden Zielfunktion optimal zu wählen.

$$
U_{J}=\max _{c_{j}, c_{j}}\left\{\tilde{u}\left(c_{j} \mid I_{J}, A_{j}, F_{j}\right)+\sum_{t=j+1}^{T}(1+\rho)^{j-t} \tilde{u}\left(c_{j} \mid I_{t}, A_{t}, F_{t}\right)\right\} \quad \text { für } j=\tau, \ldots, T
$$

16 Eine weitere Begründung ergibt sich, wenn das Arbeitseinkommen als Zins des Humankapitals interpretiert wird: Das laufende Einkommenskonto faßt dann die Zinseinkommen aus Real- und Humankapital zusammen. 
Im Gegensatz zu Gleichung 2.14 im vorangehenden Abschnitt besteht wegen der veränderten Budgetrestriktion (2.17) - einer Ungleichung - nun kein analytisch auflösbarer Zusammenhang zwischen dem realisierten Konsum $c$ und der Sustainable Consumption Variablen $c^{*}$. Das Optimum der Zielfunktion (2.18) muß daher über beide Variablen gesucht werden. Diese Suche läßt sich vereinfachen, wenn man die Entscheidung über $c$ und $c^{*}$ als hierarchische Entscheidung betrachtet, denn der funktionale Zusammenhang zwischen $C$ und $C^{*}$ in Gleichung (2.14) bedeutet, daß $c^{*}$ für ein gegebenes $c$ bestimmt wird und die Kausalität eine Richtung hat. Wenn die Kausalität auf die Zielfunktion (2.18) übertragen wird, dann kann die Optimierung in zwei Schritten erfolgen:

$$
U_{j}=\max _{c_{j}}\left\{\tilde{u}\left(c_{j} \mid I_{l}, A_{j}, F_{j}\right)+\max _{c_{j}}\left[\sum_{t=j+1}^{T}(1+\rho)^{j-t} \tilde{u}\left(c_{j}^{*} \mid I_{t}, A_{t}, F_{t}\right)\right]\right\}
$$

In jeder Periode $j=\tau, \ldots, T$-1 wird im ersten Schritt für ein gegebenes $c_{j}$ ein optimales $C_{j}^{*}$ in der innere Optimierung bestimmt, bevor im zweiten Schritt die äußere Optimierung ein optimales $c_{j}$ bestimmt. Die numerische Umsetzung dieser Optimierungsaufgabe muß die unstetige Nutzenfunktion des Mental Accounting Modells berücksichtigen. Ein effizientes Verfahren, das keine Stetigkeit voraussetzt, geht auf Brent (1973) zurück. Der Brent-Algorithmus ${ }^{17}$ verbindet parabolische Interpolation mit Golden Section Search und erreicht mit relativ wenigen Funktionsauswertungen eine hinreichende Genauigkeit.

\section{Beispielprofile}

Die theoretische Betrachtung zeigt, daß das Mental Accounting Modell Unterschiede im Verhalten von Haushalten mit verschiedenem Arbeitseinkommen erklären kann. In dem simulierten Beispiel werden zwei Haushalte gegenübergestellt, die bis auf ihr Arbeitseinkommen völlig gleich sind. Der eine Haushalt beginnt im Alter von 21 Jahren mit einem Einstiegsgehalt von $w_{21}=1$, dagegen bekommt der andere Haushalt ein Einstiegsgehalt von $w_{21}=4$. Während des Erwerbslebens steigt das Arbeitseinkommen für beide Haushalte mit abnehmender Rate an und erreicht mit dem Verrentungsalter den jeweils doppelten Wert des Einstiegsgehalts als Maximum. Im Ruhestand beziehen die Haushalte dann 25\% des letzten Arbeitslohnes - eine bewußt niedrig angesetzte Ersatzquote, die einen starken Anreiz zum Sparen schafft und so die Mechanik des Modells besonders

17 Der Brent-Algorithmus wird in seinen theoretischen und numerischen Eigenschaften in Numerical Recipes - The Art of Scientific Computing von Press et al. (1992) ausfuihrlich behandelt. 
deutlich herausstellt. Als Periodennutzen wird wieder die isoelastische Nutzenfunktion verwendet, die mit den Nutzenabschlägen $\Pi^{A}=\Pi^{F}=0.02$ zur Mental Accounting Nutzenfunktion (2.12) vervollständigt wird. Der Zinssatz und die Zeitpräferenzrate sind gleich und betragen $5 \%$. Abbildung 2.4 bildet die realisierten Konsumprofile ab; die Entwicklung der Assetkonten (Vermögensprofile) folgt in Abbildung 2.5.

Der relativ ärmere Haushalt realisiert ein flaches Konsumprofil und unterscheidet sich nicht von einem Haushalt, dessen Verhalten durch das klassische Lebenszyklusmodell beschrieben wird. Anders ist die Situation für den relativ reicheren Haushalt: Sein realisiertes Konsumprofil steigt in den ersten fünfzehn Jahren des Erwerbslebens an, bleibt dann bis zur Verrentung auf hohem Niveau konstant und springt zum Beginn des Ruhestands auf ein niedrigeres Niveau, auf dem es bis zum Lebensende verbleibt. Nach der Betrachtung der Vermögensprofile wird deutlich, warum dieser Sprung im Konsumprofil des Haushaltes mit hohem Arbeitseinkommen entsteht.

Abbildung 2.4: Mental Accounting Konsumprofile

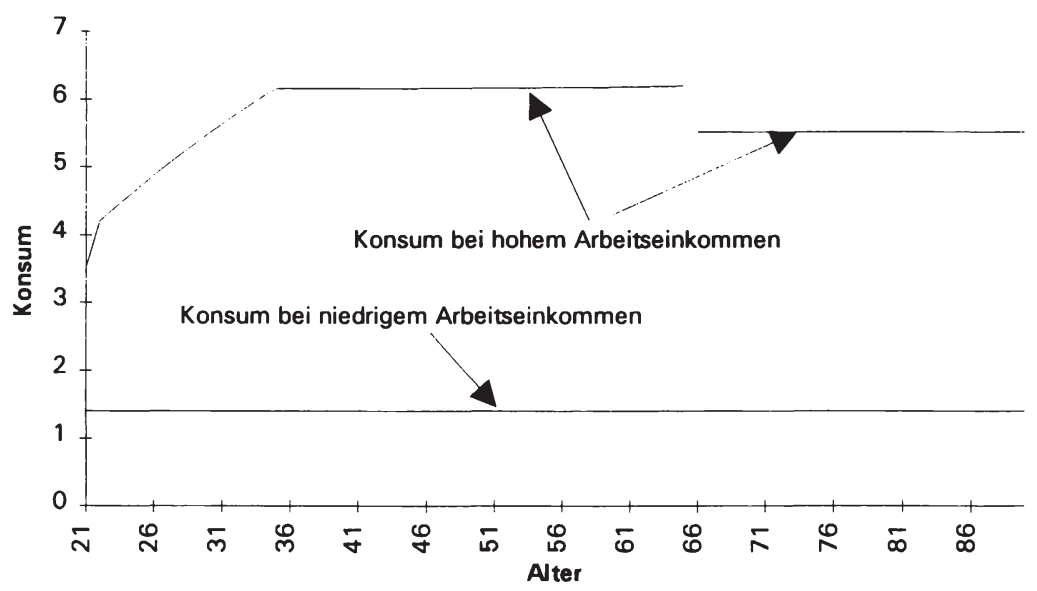

Quelle: Eigene Berechnungen

Abbildung 2.5 zeigt die Profile der Assetkonten der Beispielhaushalte. Das Profil des relativ ärmeren Haushalts entspricht dem buckelförmigen Verlauf, der für das klassische Lebenszyklusmodell typisch ist. Bis zum Alter von 35 Jahren wird ent- 
spart, wobei das zukünftige Arbeitseinkommen beliehen wird. Erst danach werden zunehmend Ersparnisse gebildet, die im Ruhestand zur Aufbesserung der Rente wieder abgebaut werden. Im 65. Lebensjahr ist das Vermögen maximal und beträgt mehr als zwölf Geldeinheiten, wodurch das Verhältnis zum maximalen Arbeitseinkommen, das ebenfalls im 65. Lebensjahr angenommen wird, gröBer als sechs ist. Ein völlig anderes Bild ergibt sich für den Haushalt mit dem höheren Einkommen: Sein Assetkonto wird zu keiner Zeit reduziert, es ist während der ersten fünfzehn Erwerbsjahre annähernd konstant und steigt dann auf ein maximales Vermögen von über siebzig Geldeinheiten an, so daß das Verhältnis von maximalen Assets zum maximalen Arbeitseinkommen (beide Maxima werden im 65. Lebensjahr erreicht) fast neun erreicht. Also hat der reichere Haushalt nicht nur absolut, sondern auch relativ zum Arbeitseinkommen ein höheres Vermögen. Während des Ruhestands wird das erreichte Niveau gehalten und der Haushalt hinterläßt am Lebensende ein ansehnliches Vermögen, obwohl er kein Vererbungsmotiv hat. Dies ist auf den ersten Blick erstaunlich, macht für Mental Accounting Haushalte jedoch Sinn: Die konstanten, für ärmere und reichere Haushalte gleichen Nutzenabschläge führen dazu, daß Entsparen für Haushalte mit hohem Arbeitseinkommen relativ zu Haushalten mit geringem Arbeitseinkommen teurer ist, denn im Verhältnis zum marginalen Nutzen zusätzlicher Konsumeinheiten haben die Nutzenabschläge für reichere Haushalte ein höheres $\mathrm{Ge}$ wicht. Also sind die reichen Haushalte daran interessiert, die geringe Altersrente ohne Entsparen aufzubessern. Die einzige Möglichkeit, mit der sie dieses Ziel in der hier vorgestellten Version des Mental Accounting Modells erreichen können, ist ein hohes Zinseinkommen - und das ist der Grund für das nach der Verrentung konstant hohe Vermögen der Haushalte mit hohem Arbeitseinkommen. Gleichzeitig wird so der plötzlich mit der Verrentung auftretende Sprung im Konsumprofil verständlich: Das Zinseinkommen reicht nicht aus, um die Rente auf das Niveau des vor der Verrentung erreichten Konsums anzuheben. Durch geringeren Konsum vor der Verrentung könnte der Haushalt natürlich einen glatten Übergang zum Konsum nach der Verrentung erreichen, dies wäre aber mit einem geringeren Lebensnutzen verbunden. Der reichere Haushalt entscheidet sich im Mental Accounting Modell bewußt für das in Abbildung 2.4 gezeigte unstetige Konsumprofil. ${ }^{18}$

18 Der in Abbildung 2.4 nicht gezeigte Verlauf der Sustainable Consumption Variable informiert den Haushalt sehr genau über den Rückgang im Konsumniveau nach der Verrentung: Je näher der Haushalt dem Verrentungsalter kommt, desto mehr nähert sich die Sustainable Consumption Variable dem nach der Verrentung konstanten Konsumniveau an. Hier wird erneut deutlich, daß die Sustainable Consumption eine vorausschauende Intention hat und den Entscheidungsträgern spätere Konsequenzen ihrer heutigen Entscheidungen aufzeigen will. 
Abbildung 2.5: Mental Accounting Vermögensprofile

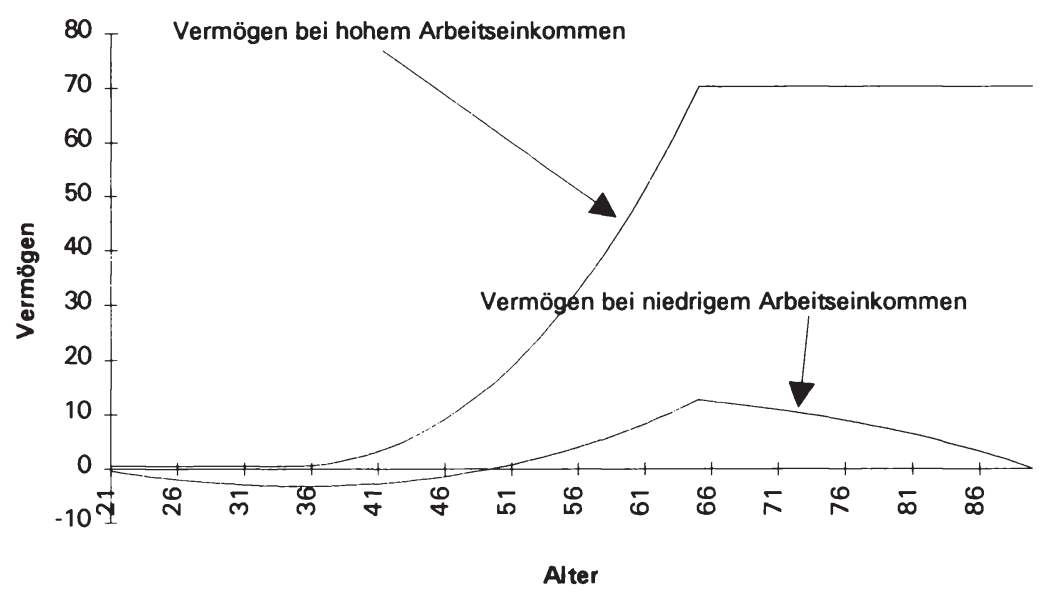

Quelle: Eigene Berechnungen

Der ärmere Haushalt steht grundsätzlich vor den gleichen Überlegungen, aber seine Entscheidung fällt zugunsten der Konsumglättung aus, denn sein marginaler Konsumnutzen ist relativ zu den Nutzenabschlägen hoch. Er löst sein Vermögen nach der Verrentung kontinuierlich auf und stirbt im 90. Lebensjahr ohne Vermögen.

Die Mechanik des Mental Accounting Modells wird durch die exogen vorgegebenen Nutzenabschläge getrieben. In der hier vorgestellten Beispielsimulation haben sie die Werte $\Pi^{A}=\Pi^{F}=0,02$. Interessant ist die Frage, welches Gewicht die Nutzenabschläge relativ zum Einkommen haben. Dazu berechne ich ein Einkommensäquivalent $x$, so daß $u(x)=0,02$ gilt, $x$ ist also der Betrag, der den Nutzenabschlag gerade kompensiert. Für die hier verwendete isoelastische Nutzenfunktion ergibt sich mit $x=5$ ein Wert, der deutlich über beiden Einstiegsgehältern liegt - damit haben die Nutzenabschläge ein sehr hohes Gewicht. Setzt man den Kompensationsbetrag $x$ in Beziehung zu den Kompensationsbeträgen in Tabelle 2.1, mit der die Güte der Sustainable Consumption Approximation gemessen wird, dann wird deutlich: Die Nutzenabschläge sind der kritische Punkt dieses Beispiels - nicht die Approximation des Optimums durch das Sustainable Consumption Modell. 


\subsubsection{Kritische Würdigung}

Die theoretische Betrachtung und die Beispielprofile zeigen, daß sich aus dem Mental Accounting Modell interessante aber auch problematische Aussagen ergeben. Das Mental Accounting Modell bietet eine Erklärung für unterschiedliches Verhalten unterschiedlicher Haushalte bei einheitlichen Präferenzen, indem es einen Keil zwischen den abnehmenden Grenznutzen des Konsums und die konstanten Nutzenabschläge treibt. Die Nutzenabschläge entstehen immer dann, wenn Teile des Konsums durch das Auflösen von Ersparnissen finanziert werden. Als Konsequenz ergeben sich im Mental Accounting Modell zwei typische Verhaltensweisen: Haushalte mit einem niedrigen Arbeitseinkommen haben ein niedriges Konsumniveau, daher ist der Nutzen zusätzlicher Konsumeinheiten für die ärmeren Haushalte so hoch, daß sie den Nutzenabschlag in Kauf nehmen. Konsumglättung erreichen die ärmeren Haushalte durch Sparen während des Erwerbslebens und Entsparen während des Ruhestands. Die Gruppe der Haushalte mit hohem Arbeitseinkommen verhält sich anders, denn zusätzliche Konsumeinheiten erhöhen ihren Konsumnutzen nur wenig, also bestimmen jetzt die Nutzenabschläge das Verhalten. Entsparen ist zu teuer (gemessen in Nutzeneinheiten), Konsumglättung wird daher über die Zinsen des Vermögens angestrebt: Die reichen Haushalte bauen während des Erwerbslebens ein hohes Vermögen auf und heben mit dessen Zinsen das Alterseinkommen auf das gewünschte Niveau. Mit diesem Argument kann das Mental Accounting Modell positives Vermögen am Lebensende erklären, unabhängig von Erbschaftsmotiven.

Die Beispielprofile verdeutlichen auch die Probleme des Mental Accounting Modells. Ein deutlicher Sprung im Konsumprofil erscheint ebenso wie das ab der Verrentung konstante Vermögensprofil der reicheren Haushalte wenig plausibel. Hier ist die Mechanik der Nutzenabschläge zu starr, denn es gibt keinen Zusammenhang zwischen Nutzenabschlägen auf der einen Seite und dem Konsum, Arbeitseinkommen und Vermögen auf der anderen Seite. Läßt man je nach Einkommen unterschiedliche, aber weiterhin exogene Nutzenabschläge zu, so würde das Mental Accounting Modell zwar flexibler, aber nicht weniger problematisch. Denn wenn es Gründe für einen Zusammenhang zwischen den Nutzenabschlägen und den ökonomischen Verhältnissen der Haushalte gibt, dann sollte dieser $\mathrm{Zu}$ sammenhang auch endogen berücksichtigt werden. Doch mit dieser Überlegung gehe ich über das Mental Accounting Modell von Shefrin und Thaler hinaus; bei Shefrin und Thaler sind die Nutzenabschläge exogen.

Eine wichtige Frage beschließt den Abschnitt: Ist das Mental Accounting Modell geeignet, das Haushaltsverhalten im allgemeinen Gleichgewichtsmodell $\mathrm{zu}$ beschreiben? Die wirtschaftspolitisch relevante Frage der vorliegenden Arbeit konzentriert sich auf die gesamtwirtschaftlichen Auswirkungen des demographischen Wandels. Der demographische Wandel ist gleichbedeutend mit Verschiebungen 
in der Kohortenverteilung: Die älteren Kohorten werden an Gewicht gewinnen, die jüngeren Kohorten werden an Gewicht verlieren. Ein sinnverwandter Titel für die vorliegende Arbeit könnte daher lauten: "Welche gesamtwirtschaftlichen Auswirkungen ergeben sich aus den Veränderungen in der Kohortenverteilung?" Das Haushaltsmodell muß sich an dieser Frage orientieren und die Haushalte $\mathrm{zu}$ repräsentativen Haushalten je Kohorte zusammenfassen. Repräsentative Haushalte zur Beschreibung des Kohortenverhaltens machen aber nur dann Sinn, wenn das Haushaltsmodell genug Raum für kohortenspezifisches Verhalten bietet. Im klassischen Lebenszyklusmodell ist dieser Raum sehr eng: Die Kohorten können sich nur im Niveau ihres Konsumprofils unterscheiden, der Verlauf des Konsumprofils (die intertemporale Allokation des Konsums) ist für alle Kohorten gleich. Das Mental Accounting Modell ist etwas flexibler, es kann für unterschiedliche Kohorten nicht nur unterschiedliche Niveaus, sondern auch unterschiedliche Verläufe der Konsumprofile erklären. Den Variationsmöglichkeiten sind bei exogen gegebenen Nutzenabschlägen allerdings enge Grenzen gesetzt, denn es gibt nur zwei Verhaltensmuster: das „klassische“, buckelförmige Sparprofil und das konstante Altersvermögen. Einen kontinuierlichen Übergang zwischen den beiden Verhaltensmustern gibt es bei konstanten Nutzenabschlägen nicht. Damit ist das Mental Accounting Modell für die Beschreibung kohortenspezifischen Haushaltsverhaltens ungeeignet, denn es ist wenig realistisch, daß sich zwei aufeinanderfolgende Kohorten völlig unterschiedlich verhalten.

Die Beschäftigung mit dem Mental Accounting Modell war aber keineswegs wertlos. Das Sustainable Consumption Konzept als Optimierungsverfahren wurde erfolgreich in einen neuen Zusammenhang gestellt. Und das Mental Accounting Modell bietet zwei Kritikpunkte, die im nächsten Abschnitt aufgegriffen werden und das klassische Lebenszyklusmodell erweitern:

- Wenn die Haushalte sich nach der Regel „Greife dein Vermögen nur in Ausnahmesituationen an "verhalten, dann haben sie offensichtlich irgendeinen Nutzen aus ihrem Vermögen. Zur Begründung der Nutzenabschläge spielt diese Verhaltensregel im Mental Accounting Modell eine entscheidende Rolle, aber eine nutzentheoretische Begründung für diese Regel wird nicht diskutiert.

- Die Nutzenabschläge sind im Mental Accounting Modell exogen gegeben. Es gibt also keinen Zusammenhang zwischen Nutzenabschlägen sowie Konsum, Einkommen und Vermögen.

Im folgenden Abschnitt werden diese beiden Punkte wieder zur Sprache kommen, dann allerdings in einem neoklassischen Lebenszyklusmodell, das um ein Erbschaftsmotiv bei unsicherer Lebenserwartung erweitert ist. Die Diskussion wird zeigen, daß die Nutzenabschläge in diesem Modell gewissermaßen endogenisiert werden. 


\subsection{Das Erbschaftsnutzenmodell}

Sowohl im klassischen Lebenszyklusmodell als auch im Mental Accounting Modell ist der Konsum die einzige nutzenstiftende Variable. Das Vermögen hat in diesen Modellen nur einen mittelbaren Einfluß auf den Nutzen - es ermöglicht die Konsumglättung. Die Erweiterung des klassischen Lebenszyklusmodells um ein additiv-separables Erbschaftsmotiv ändert daran wenig, solange von sicherer Lebenserwartung ausgegangen wird. Zwar geht nun das am Lebensende vorhandene Vermögen als Erbschaft direkt in den Lebensnutzen ein, doch die Eulergleichung - und damit die intertemporale Allokation des Konsums - bleibt unverändert ${ }^{19}$. Die Simulationsmodelle von Auerbach et al. (1989), Raffelhüschen und Kitterer (1990) sowie Buslei und Kraus (1996) verwenden das Lebenszyklusmodell mit Erbschaftsmotiv bei sicherer Lebenserwartung zur Beschreibung des Sparverhaltens der Haushalte. Sichere Lebenserwartung heißt, da $\beta$ alle Haushalte das vorgegebene maximale Alter erreichen und erst dann ihr Vermögen vererben. Diese Annahme ist für die numerische Umsetzung des Modells praktisch, aber nicht realistisch. Tatsächlich gibt es in jedem Lebensjahr eine Wahrscheinlichkeit zu sterben, also gibt es auch in jedem Lebensjahr eine Wahrscheinlichkeit, eine Erbschaft zu hinterlassen. Im Zusammenhang mit dem demographischen Wandel sind das zwei entscheidende Punkte, die betont werden müssen:

- Der demographische Wandel wird nicht zuletzt durch eine Veränderung der Sterbewahrscheinlichkeit beeinflußt.

- Die Veränderung der Relation zwischen jungen und alten Kohorten verändert die Erbschaften, denn wenn vielen Alten wenig Junge gegenüberstehen, dann sind die Pro-Kopf-Erbschaften der Jungen möglicherweise relativ hoch.

Lebenszyklusmodelle mit Erbschaftsmotiv bei unsicherer Lebenserwartung erlauben daher eine realitätsnähere Beschreibung des Sparverhaltens der Haushalte, gerade unter dem Eindruck des demographischen Wandels. Die Idee, ein Erbschaftsmotiv bei unsicherer Lebenserwartung zu betrachten, geht zurück auf die grundlegende Arbeit von Yaari (1965). Ausgehend von seiner Arbeit und der Arbeit von Hurd (1989) werde ich im folgenden ein Erbschaftsnutzenmodell vorstellen, das ich im dritten Kapitel in mein Simulationsmodell integrieren werde. Gegenüber früheren Arbeiten ist das ein wesentlicher Fortschritt, denn mit den Sterbewahrscheinlichkeiten nehme ich einen zusätzlichen, wichtigen Aspekt des demographischen Wandels auf.

19 Wegen der Separabilitätsannahme wird die optimale Erbschaft in einer eigenen Optimalitätsbedingung bestimmt, unabhängig vom Konsumprofil. 


\subsubsection{Theoretische Betrachtung}

Das Erbschaftsnutzenmodell ist eine Erweiterung des klassischen Lebenszyklusmodells um einen Erbschaftsnutzen bei unsicherer Lebenserwartung. Der Grundgedanke ist schnell zu begreifen: Wenn eine Person ${ }^{20}$ die Periode $t$ lebend erreicht, dann steigt ihr Lebensnutzen um $u\left(c_{t}\right)$, aber wenn die Person am Ende der Vorperiode $t-1$ gestorben ist, dann hinterläßt sie ihren Erben das Vermögen, das sie am Ende der Periode $t-1$ besessen hat. Weil die Vermögensvariable $a$ das Vermögen am Anfang einer Periode bezeichnet, beläuft sich das Vermögen der Person am Ende der Periode $t-1$ auf $\left(1+r_{t-1}\right) \cdot a_{t-1}+w_{t-1}-c_{t-1}$, das nach der Budgetrestriktion (2.2) gerade das Vermögen $a_{t}$ am Anfang der Periode $t$ ist. Stirbt die Person am Ende von Periode $t-1$, dann steigt ihr Lebensnutzen um den Erbschaftsnutzen $v\left(a_{t}\right)$. Der erwartete Nutzen zukünftiger Perioden $t$ besteht daher aus zwei Komponenten: erstens dem Konsumnutzen $u\left(c_{t}\right)$ und zweitens dem Erbschaftsnutzen $v\left(a_{t}\right)$. Diese beiden Komponenten werden mit den entsprechenden Eintrittswahrscheinlichkeiten gewichtet, wobei zu beachten ist, daß die Wahrscheinlichkeiten von der Planungsperiode $\tau$ abhängen.

Um diese auf das Erleben der Periode $\tau$ bedingten Wahrscheinlichkeiten anzugeben, gehe ich aus von der Wahrscheinlichkeit $m_{t}$ eines Neugeborenen im Alter von $t$ Jahren zu sterben. Die Summe über die $m_{t}$ ist eins:

$$
\sum_{t=1}^{\infty} m_{t}=1
$$

Wenn die Person das Alter $\tau$ lebend erreicht, d.h. sie ist bis zum Alter $\tau-1$ nicht gestorben, dann ist die Wahrscheinlichkeit $m_{t} \tau$ im Alter $t>\tau$ zu sterben aus der entsprechenden Wahrscheinlichkeit eines Neugeborenen, bedingt auf das Überleben bis zur Periode $\tau-1$, zu errechnen:

$$
m_{t}^{\tau}=\frac{m_{t}}{1-\sum_{l=1}^{\tau-1} m_{l}}=\frac{m_{t}}{\sum_{l=\tau}^{\infty} m_{l}}
$$

Aus den $m_{t}{ }^{\tau}$ ergibt sich die Wahrscheinlichkeit $s_{t}^{\tau}$, bis zum Alter $t$ zu überleben, gegeben die Person ist bis zum Alter $\tau$ - 1 nicht gestorben:

20 Vorerst unterscheide ich nicht zwischen einzelnen Personen und Haushalten. Im dritten Kapitel werde ich diesen wichtigen Punkt aufgreifen und das Modell explizit als Haushaltsmodell formulieren. 


$$
s_{t}^{\tau}=1-\sum_{i=\tau}^{t} m_{i}^{\tau}=\sum_{i=t+1}^{\infty} m_{i}^{\tau}=\frac{\sum_{l=t+1}^{\infty} m_{i}}{\sum_{i=\tau}^{\infty} m_{i}}
$$

Bei dieser Fortschreibung der Wahrscheinlichkeiten unterstelle ich, daß außer dem Erreichen des Alters $\tau$ keine neuen Informationen bezüglich der Sterbewahrscheinlichkeit hinzukommen. Zum Beispiel wird die plötzliche Feststellung einer schweren Krankheit durch die Fortschreibung der Sterbewahrscheinlichkeiten nicht erfaßt, denn die plötzlich offenbarte Krankheit verändert die zu Grunde liegende Verteilung der $m_{t}$ für $t>\tau$.

Mit den auf die Planungsperiode $\tau$ bedingten Überlebens- und Sterbewahrscheinlichkeiten kann der erwartete Lebensnutzen aus Konsum und Erbschaften angegeben werden:

$$
E_{\tau}(U)=\sum_{t=\tau}^{\infty}(1+\rho)^{\tau-t}\left[s_{t}^{\tau} u\left(c_{t}\right)+m_{t}^{\tau} v\left(a_{t}\right)\right]
$$

Die erwartete Lebensnutzenfunktion (2.23) entspricht den Modellen von Yaari (1965) und Hurd (1989). Allerdings beschränkt Hurd den marginalen Erbschaftsnutzen auf eine konstante Funktion, diese Beschränkung werde ich durch eine allgemeinere Erbschaftsnutzenfunktion aufheben.

Die unendliche Summe in Gleichung (2.23) ist in der Realität endlich, denn die Überlebenswahrscheinlichkeit für Personen, die deutlich über hundert Jahre alt sind, ist praktisch null. Wenn $T$ das Alter bezeichnet, mit dem die Überlebenswahrscheinlichkeit kleiner als ein beliebiges gegebenes $\varepsilon$ wird, dann kann der erwartete Lebensnutzen durch eine endliche Summe mit beliebiger Genauigkeit approximiert werden:

$$
\begin{aligned}
E_{\tau}(U) & =\sum_{t=\tau}^{T}(1+\rho)^{\tau-t}\left[s_{t}^{\tau} u\left(c_{t}\right)+m_{t}{ }^{\tau} v\left(a_{t}\right)\right] \\
& =\sum_{t=\tau}^{T-1}(1+\rho)^{\tau-t}\left[s_{t}^{\tau} u\left(c_{t}\right)+m_{t}{ }^{\tau} v\left(a_{t}\right)\right]+(1+\rho)^{\tau-T} m_{T}{ }^{\tau} v\left(a_{T}\right)
\end{aligned}
$$

Die zweite Zeile ergibt sich, weil nach Formel (2.22) $s_{T} \tau$ für alle $\tau$ gleich null ist. Damit wird das geplante Endvermögen $a_{T}$ in Gleichung (2.24) endogen. Das Anfangsvermögen $a_{1}$ ist weiterhin exogen gegeben. Die Optimierung des erwarteten Lebensnutzens unter Beachtung der um die erhaltenen Erbschaften und einen variablen Zinssatz erweiterten Budgetrestriktion (2.2) ergibt zwei Optimalitätsbedingungen: eine Eulergleichung, die nicht nur das Konsum-, sondern auch das 
Vermögensprofil im Optimum beschreibt, und eine Bedingung für das optimale Endvermögen $a_{T}$ :

$$
\frac{\left(1+r_{t}\right)}{(1+\rho)} \frac{s_{t}^{\tau}}{s_{t-1}^{\tau}} u^{\prime}\left(c_{t}\right)+\frac{1}{1+\rho} \frac{m_{t}^{\tau}}{s_{t-1}{ }^{\tau}} v^{\prime}\left(a_{t}\right)=u^{\prime}\left(c_{t-1}\right) \quad \text { für } t=\tau+1, \ldots, T-1
$$

$$
\frac{m_{T}{ }^{\tau}}{s_{T-1}{ }^{\tau}} v^{\prime}\left(a_{T}\right)=(1+\rho) u^{\prime}\left(c_{T-1}\right)
$$

Die nähere Betrachtung der Quotienten aus Sterbe- und Überlebenswahrscheinlichkeiten liefert eine wichtige Erkenntnis, denn es gilt:

$$
\frac{s_{t}^{\tau}{ }^{\tau}}{s_{t-1}{ }^{\tau}}=\frac{\sum_{i=t+1}^{\infty} m_{i}}{\sum_{i=t}^{\infty} m_{i}}=1-\frac{m_{t}}{1-\sum_{i=1}^{t-1} m_{i}}=1-q_{t}
$$

$$
\frac{m_{t}^{\tau}}{s_{t-1}{ }^{\tau}}=\frac{m_{t}}{1-\sum_{i=1}^{t-1} m_{i}}=q_{t}
$$

Beide Quotienten sind unabhängig von der Planungsperiode $\tau$, es sind HazardRaten. (2.28) ist die Wahrscheinlichkeit, im Alter $t$ zu sterben, gegeben man hat bis zum Alter $t-1$ überlebt. Diese hier mit $q_{t}$ bezeichnete Hazard-Rate ist der in den Sterbetafeln veröffentlichte Wert. Die Hazard-Rate (2.27) ist das entsprechende Gegenereignis: Die Wahrscheinlichkeit in Periode $t$ zu überleben, gegeben man hat bis zum Alter $t$ - 1 überlebt. Weil die Optimalitätsbedingungen unabhängig vom Planungszeitpunkt $\tau$ sind, ist das Erbschaftsnutzenmodell dynamisch konsistent, also reicht es, am Lebensanfang einmal zu optimieren. In späteren Perioden muß dann nur noch dem optimalen Plan gefolgt werden. Eine erneute Optimierung wäre nur dann nötig, wenn sich die Informationen in einer nicht vorausgesehenen Weise ändern. Diesen Fall schließe ich in meinem Modell aus, indem ich rationale Erwartungen unterstelle und von Informationsschocks absehe.

Mit der Hazard-Rate $q$ aus den Sterbetafeln vereinfachen sich die Optimalitätsbedingungen des Erbschaftsnutzenmodells zu der folgenden Gleichung, die wegen $\left(1-q_{T}\right)=0$ auch die Bedingung für das Endvermögen enthält:

$$
\frac{\left(1+r_{t}\right)}{(1+\rho)} \cdot\left(1-q_{t}\right) \cdot u^{\prime}\left(c_{t}\right)+\frac{1}{1+\rho} \cdot q_{t} \cdot v^{\prime}\left(a_{t}\right)=u^{\prime}\left(c_{t-1}\right) \quad \text { für } t=\tau+1, \ldots, T
$$


Bevor ich die Optimalitätsbedingungen weiter interpretiere, kläre ich, ob das Erbschaftsnutzenmodell eine eindeutige Lösung besitzt. Die Antwort ist ja, wenn zwei Voraussetzungen erfüllt sind:

- Der marginale Konsumnutzen $u^{\prime}$ ist positiv und eineindeutig definiert für alle positiven Konsumniveaus $\boldsymbol{C}$.

- Der marginale Erbschaftsnutzen $v^{\prime}$ ist nicht negativ für alle positiven und negativen Vermögen $a$.

Die erste Bedingung wird von Konsumnutzenfunktionen typischerweise erfüllt. Die Beschränkung auf die positiven reellen Zahlen ist unproblematisch, weil ein „negativer“ Konsum nicht sinnvoll interpretiert werden kann. Dagegen beschreibt der Erbschaftsnutzen die Präferenzen für Vermögen, das bei verschuldeten Personen negativ ist. Welchen Nutzen (oder Leid) eine verschuldete Person aus dem Wissen hat, ihren Erben bei ihrem Tod Schulden zu hinterlassen, wird durch die jeweilige Spezifikation des Erbschaftsnutzens festgelegt. Die obige Bedingung an den Erbschaftsnutzen verlangt nur, daß der marginale Erbschaftsnutzen positiv sein muß, also darf weniger Vermögen den Erbschaftsnutzen nicht erhöhen. Der marginale Erbschaftsnutzen kann für große negative Vermögen gegen null gehen, er kann aber auch konstant bleiben oder weiter zunehmen. Wenn ich für die Simulation eine Erbschaftsnutzenfunktion spezifiziere, komme ich auf diese Diskussion zurück.

Der Existenzbeweis ist konstruktiv, d.h. der Beweis liefert zugleich ein Berechnungsschema für die eindeutige Lösung der Optimierungsaufgabe.

1. Beginne mit einem beliebigen $a_{T}$ als Startwert.

2. Berechne für gegebenes $a_{T}$ mit Gleichung (2.29) $c_{T-1}$ Die Lösung $c_{T-1}$ ist eindeutig, weil $v^{\prime}$ positiv ist und $u^{\prime}$ eineindeutig auf der Menge der positiven Zahlen definiert ist.

3. Berechne mit der Budgetrestriktion (2.2) für gegebene $a_{t}, c_{t-1}$ das Vermögen

$$
a_{t-1}=\frac{1}{1+r_{t-1}}\left(a_{t}-w_{t-1}+c_{t-1}\right)
$$

4. Setze $c_{t-1}, a_{t-1}$ in Gleichung (2.29) ein und berechne $c_{t-2}$. Die Lösung $c_{t-2}$ ist eindeutig, weil $u^{\prime}$ und $v^{\prime}$ positiv sind (und damit die linke Seite von Gleichung (2.29) positiv ist) und $u^{\prime}$ eineindeutig auf der Menge der positiven Zahlen definiert ist.

5. Wenn die Anfangsperiode $\tau$ noch nicht erreicht ist, gehe zurück zu Schritt 3. 
6. Wenn $a_{1}$ vom vorgegebenen Anfangsvermögen abweicht, gehe mit einem neuen Startwert $a_{T}$ zurück zu Schritt 2 . Wenn $a_{1}$ dem vorgegebenen Anfangsvermögen gleicht, ist die Lösung der Optimierungsaufgabe eindeutig bestimmt.

Der Existenzbeweis stellt fest, daß das Erbschaftsnutzenmodell eine eindeutige Lösung hat, also kann ich nun überlegen, welche Aussagen aus der Optimalitätsbedingung (2.29) abgeleitet werden können. Ausgangspunkt meiner Überlegungen ist das klassische Lebenszyklusmodell, das als Spezialfall mit $v^{\prime}=0$ für alle $a$ im Erbschaftsnutzenmodell enthalten ist und dessen Ergebnisse mit den Implikationen des Erbschaftsnutzenmodells verglichen werden. Zwei Fragen, auf die das klassische Lebenszyklusmodell keine befriedigende Antwort geben kann, stelle ich in den Mittelpunkt der Diskussion:

- Wodurch wird die intertemporale Allokation des Konsums bestimmt?

- Welchen Verlauf nehmen Konsum- und Vermögensprofil im Alter?

Beide Fragen untersuche ich in diesem Abschnitt theoretisch mit Hilfe der Optimalitätsbedingungen des Erbschaftsnutzenmodells. Im nächsten Abschnitt veranschauliche ich die Aussagen des Modells mit einigen Beispielprofilen.

\section{Intertemporale Allokation}

Im Optimum des klassischen Lebenszyklusmodells entspricht der entgangene Nutzen einer marginalen Konsumeinheit in der Periode $t$ genau dem abdiskontierten zusätzlichen Nutzen einer marginalen Konsumeinheit in der Folgeperiode $t+1$. Genau die gleiche Abwägung zwischen entgangenem und zusätzlichem Nutzen bringt die Eulergleichung (2.29), in der zum Konsumnutzen der Erbschaftsnutzen hinzukommt. Wenn der Konsum in Periode $t$ um eine marginale Einheit eingeschränkt wird, dann sinkt der Nutzen in $t$ um $u^{\prime}\left(c_{t}\right)$. Gleichzeitig wächst das Vermögen der Folgeperiode $t+1$ um eine marginale Einheit und erhöht den Erbschaftsnutzen um $v^{\prime}\left(a_{t}\right)$. Das größere Vermögen erhöht aber auch die Konsummöglichkeiten in der Zukunft, so daß der Konsumnutzen ebenfalls steigen kann. Das Optimum ist genau dann erreicht, wenn die Person indifferent ist zwischen dem Nutzen einer marginalen Konsumeinheit in Periode $t$ und dem Konsum- und Erbschaftsnutzen, den diese Einheit in Periode $t+1$ stiftet. Diese Wirkungsweise wird besonders deutlich, wenn die Eulergleichung (2.29) ein wenig umgeformt wird:

$$
\left[-\frac{1}{1+\rho}\left(1-q_{t}\right) \frac{u^{\prime}\left(c_{t}\right)}{u^{\prime}\left(c_{t-1}\right)}\right]+\frac{1}{1+r_{t}}\left[-\frac{1}{1+\rho} q_{t} \frac{v^{\prime}\left(a_{t}\right)}{u^{\prime}\left(c_{t-1}\right)}\right]=-\frac{1}{1+r_{t}}
$$


Der erste Term in eckigen Klammern auf der linken Seite ist die marginale Rate der Substitution (MRSC) beim Tausch von Konsumeinheiten in Periode $t-1$ gegen Konsumeinheiten in Periode $t$. Mit E(U) als Lebensnutzenfunktion (2.24) ist die MRSC definiert als:

$$
\operatorname{MRSC}_{t}=\frac{d c_{t-1}}{d c_{t}}=-\frac{\partial E(U) / \partial c_{t}}{\partial E(U) / \partial c_{t-1}}
$$

Die MRSC gibt die Antwort auf folgende Überlegung: Der Konsum $c_{t}$ wird um eine Einheit erhöht. Um wieviel Einheiten kann der Konsum in $c_{t-1}$ gesenkt werden, damit der ursprüngliche Lebensnutzen unverändert bleibt? Eine hohe MRSC bedeutet, daß zusätzlicher Konsum in Periode $t$ teuer ist, also bei konstantem Lebensnutzen nur durch eine starke Konsumeinschränkung in Periode $t-1$ erkauft werden kann.

Der zweite Term auf der linken Seite der Gleichung ist eine weitere marginale Rate der Substitution, die mit MRSA abgekürzt wird und angibt, auf wieviel Konsumeinheiten in Periode $t-1$ ohne Nutzeneinbuße verzichtet werden kann, wenn das Vermögen in $t$ um eine Einheit steigt. Die Optimalitätsbedingung (2.29) kann nun durch die marginalen Raten der Substitution beschrieben werden:

$$
M R S C_{t}+\frac{1}{1+r_{t}} M R S A_{t}=-\frac{1}{1+r_{t}}
$$

Im klassischen Lebenszyklusmodell stiftet das Vermögen keinen direkten Nutzen, also ist die MRSA = 0 und die MRSC muß im Optimum für alle Personen - unabhängig von ihrem Einkommen und Vermögen - den gleichen Wert haben. Das Erbschaftsnutzenmodell ist allgemeiner, denn nach Gleichung (2.32) besteht ein Zusammenhang zwischen der MRSC und der MRSA, also hängt das Konsumprofil (beschrieben durch die MRSC) direkt vom Vermögensprofil (beschrieben durch die MRSA) ab. Ceteris paribus gilt: Je größer der Konsum in Periode $t-1$, desto größer ist die $\mathrm{MRSA}_{t}$, also nimmt der Unterschied zwischen dem Konsumprofil im klassischen Lebenszyklusmodell und dem Konsumprofil im Erbschaftsnutzenmodell (ceteris paribus) bei steigendem Konsum zu. Im Umkehrschluß folgt, je kleiner der Konsum in $t-1$, desto geringer die $\mathrm{MRSA}_{t}$ und desto geringer die Unterschiede zwischen den Konsumprofilen im Erbschaftsnutzenmodell und dem klassischen Lebenszyklusmodell. Personen mit geringem Lebenseinkommen versuchen also auch im Erbschaftsnutzenmodell, ihren Konsum weitgehend zu glätten.

Durch die MRSA in Gleichung (2.32) bekommt das Einkommensprofil einen Einfluß auf die intertemporale Allokation des Konsums. Ein hohes Einkommen 
am Anfang des Erwerbslebens erlaubt einen schnellen Vermögensaufbau, so daß der marginale Erbschaftsnutzen (bei einer Spezifikation mit abnehmendem marginalem Erbschaftsnutzen) schneller klein wird als bei einem niedrigen Einkommen. Also hat das Einkommensprofil - die zeitliche Verteilung des Einkommens - im Erbschaftsnutzenmodell einen Einfluß auf das Konsumprofil. Zwei Einkommensprofile, die zum Beginn des Erwerbslebens den gleichen Barwert haben, sich aber in ihrem Verlauf unterscheiden, führen im Erbschaftsnutzenmodell zu unterschiedlichen Konsumprofilen. Im klassischen Lebenszyklusmodell zählt dagegen nur der Barwert des Einkommens, seine zeitliche Verteilung hat keinen Einfluß auf die zeitliche Verteilung des Konsums.

Die Optimalitätsbedingungen des Erbschaftsnutzenmodells können im Gegensatz zu der Optimalitätsbedingung des klassischen Lebenszyklusmodells unterschiedliches Verhalten für unterschiedliche Einkommensklassen und -profile erklären, denn zusätzlich zum Zinssatz, der Zeitpräferenzrate und dem marginalen Konsumnutzen spielt im Erbschaftsnutzenmodell der vom Vermögen bestimmte marginale Erbschaftsnutzen eine Rolle. Damit ist die erste Frage, auf die das klassische Lebenszyklusmodell keine befriedigende Antwort geben kann, im Erbschaftsnutzenmodell besser gelöst.

Konsum- und Vermögensprofile im Alter

Die zweite Frage, auf die das klassische Lebenszyklusmodell keine befriedigende Antwort geben kann, ist der Verlauf der Konsum- und Vermögensprofile im Alter: Das für das klassische Lebenszyklusmodell so typische hump-shaped Vermögensprofil ist empirisch schwer zu halten (siehe die Diskussion im Abschnitt 2.1). Welche Implikationen ergeben sich für den Konsum und das Vermögen im Alter aus dem Erbschaftsnutzenmodell? Um die Frage zu beantworten, betrachte ich erneut die marginalen Raten der Substitution. Die MRSA hängt von $\left(1-q_{t}\right)$ $\mathrm{ab}$, die Wahrscheinlichkeit, das Jahr $t$ zu überleben, gegeben man hat bis $t-1$ überlebt, sinkt aber mit dem Alter und erreicht mit $T$ schließlich den Wert null. Umgekehrt steigt $q_{t}$ mit zunehmendem Alter an und erreicht mit $T$ den Wert eins. Weil die MRSA von $q$ abhängt, verändert sich durch die zunehmende Sterbewahrscheinlichkeit das relative Gewicht zwischen MRSC und MRSA: Mit zunehmendem Alter wird ceteris paribus der Betrag der MRSA größer, während der Betrag der MRSC abnimmt. Also weicht das optimale Konsumprofil im Erbschaftsnutzenmodell mit zunehmendem Alter stärker vom optimalen Konsumprofil im klassischen Lebenszyklusmodell ab.

Bevor ich mit einigen Beispielprofilen die theoretische Diskussion veranschauliche, verdeutliche ich den Unterschied zwischen dem Erbschaftsnutzenmodell und dem klassischen Lebenszyklusmodell mit Erbschaftsmotiv bei sicherer Lebenser- 
wartung, denn Modelle dieses Typs beschreiben den Haushaltssektor in anderen Simulationen, beispielsweise Auerbach et al. (1989), Raffelhüschen und Kitterer (1990), sowie Buslei und Kraus (1996). Bei sicherer Lebenserwartung kommt das Erbschaftsmotiv nur im letzten Lebensjahr $T$ zum Tragen. In allen Jahren vor $T$ wird nur über den Konsum optimiert und daher ist die Eulergleichung bis auf das letzte Lebensjahr unabhängig vom Vermögen: Es ist die gleiche Eulergleichung wie im klassischen Lebenszyklusmodell (Gleichung 2.3). Das am Lebensende wirksame Erbschaftsmotiv senkt das Konsumprofil lediglich auf ein niedrigeres Niveau, denn ein Teil des Lebenseinkommens wird nun vererbt. Die intertemporale Allokation des Konsums ändert sich in diesen Lebenszyklusmodellen durch das Erbschaftsmotiv nur indirekt über die Budgetrestriktion. Dagegen greift das Erbschaftsnutzenmodell durch die MRSA während des ganzen Lebens in die intertemporale Allokation ein: Die Optimalitätsbedingung in der Schreibweise (2.32) zeigt den direkten Einfluß des Vermögensprofils auf das Konsumprofil. Weil das Erbschaftsnutzenmodell sowohl das klassische Lebenszyklusmodell $\left(v^{\prime}=0\right.$ für alle a) als auch das Erbschaftsmotiv bei sicherer Lebenserwartung $\left(s_{t}=1\right.$ für $t<T$ und $s_{T}=0$ ) als Spezialfälle enthält, kann es als gemeinsames Obermodell mit erheblich allgemeineren Aussagen betrachtet werden. Kurz zusammengefaßt lauten die Hauptergebnisse der theoretischen Betrachtung des Erbschaftsnutzenmodells wie folgt:

- Personen mit geringem Einkommen und Vermögen versuchen, ein möglichst flaches Konsumprofil zu verwirklichen.

- Mit steigendem Einkommen und zunehmendem Alter weicht das Konsumprofil im Erbschaftsnutzenmodell vom klassischen Lebenszyklusmodell ab.

- Neben der Höhe des Lebenseinkommens hat auch die zeitliche Verteilung des Einkommens einen Einfluß auf die intertemporale Allokation des Konsums.

\subsubsection{Beispielprofile}

Mit einigen Beispielen verdeutliche ich in diesem Abschnitt die Ergebnisse der theoretischen Betrachtung. Um einen Vergleich mit den Mental Accounting Profilen aus Abschnitt 2.3.3 zu ermöglichen, verwende ich für die Beispielprofile wieder die isoelastische Funktion für den Konsumnutzen und setzte $\gamma=3$; der Parameter $\gamma$ mißt jetzt den Grad der Mortalitäts-Risikoaversion. Zinssatz und Zeitpräferenzrate bleiben ebenfalls unverändert und betragen jeweils $5 \%$. Für die Hazard-Raten $q$ verwende ich den Mittelwert aus den Werten für Männer und Frauen aus der abgekürzten Sterbetafel 1989/91 des Statistischen Bundesamtes. Weil die Hazard-Rate $q$ im Alter von 100 Jahren noch nicht größer als 0,39 ist, schreibe ich sie für höhere Alter mit einer quadratischen Funktion des Alters fort, bis 
sie mit 120 Jahren den Wert 1 erreicht. ${ }^{21}$ Diese Fortschreibung ist notwendig, weil die unendliche Summe in Gleichung (2.32) nur dann mit hoher Genauigkeit durch eine endliche Summe - Gleichung (2.24) - approximiert werden kann, wenn die Hazard-Rate $q_{T}$ hinreichend nahe bei eins liegt.

Im Erbschaftsnutzenmodell ist der Erbschaftsnutzen zu spezifizieren, der nach der Voraussetzung für den Existenzbeweis zwei Eigenschaften erfüllen muß: Der marginale Erbschaftsnutzen muß positiv und für alle reellen Zahlen definiert sein. Funktionen mit diesen Eigenschaften können aus Verteilungsfunktionen gewonnen werden, die an der y-Achse gespiegelt werden. In den Beispielprofilen verwende ich für den Erbschaftsnutzen eine Funktion, die aus der logistischen Verteilungsfunktion abgeleitet ist:

$$
v(a)=-\xi \ln (1+\exp (-a)) \quad \text { mit } \xi>0
$$

Die Ableitung dieser Funktion, der marginale Erbschaftsnutzen, ist die gespiegelte logistische Verteilungsfunktion, die mit dem Parameter $\xi$ skaliert ist:

$$
v^{\prime}(a)=\frac{\xi}{1+\exp (a)}
$$

Weil der marginale Erbschaftsnutzen und nicht der Erbschaftsnutzen in die Optimalitätsbedingungen eingeht, zeigt Abbildung 2.6 den marginalen Erbschaftsnutzen. In den folgenden Beispielprofilen verwende ich einheitlich den Parameter $\xi=10$, hier zeige ich zum Vergleich auch $\xi=1$.

Der Parameter $\xi$ bestimmt das Niveau des marginalen Erbschaftsnutzens, hat aber keinen Einfluß auf die Symmetrie der Funktion. Abbildung 2.6 zeigt, da $\beta$ sich der marginale Erbschaftsnutzen in einem symmetrisch um null verteilten Intervall deutlich verändert, während er außerhalb dieses Intervalls, also für hohe Vermögen und hohe Schulden, im wesentlichen konstant ist. Der Parameter $\xi$ bestimmt erstens die Breite des Intervalls, in dem der marginale Erbschaftsnutzen schnell kleiner wird, und zweitens den konstanten marginalen Erbschaftsnutzen hoher Schulden, der gerade $\xi$ beträgt.

21 Ich identifiziere das Polynom zweiten Grades mit drei Bedingungen: Das Polynom bildet den Wert der Hazard-Rate im Alter von 100 Jahren aus der Sterbetafel ab, das Polynom liefert für das Alter von 120 Jahren den Wert eins und die Steigung des Polynoms bei 100 Jahren ist der Unterschied in den Hazard-Raten im Alter von 100 und 99 Jahren. 
Abbildung 2.6: Marginaler Erbschaftsnutzen

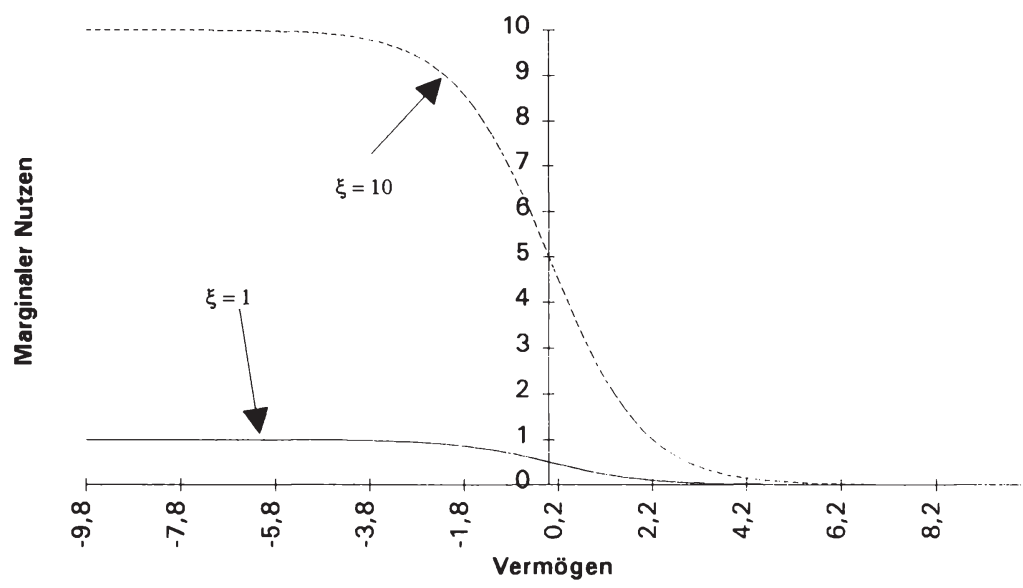

Quelle: Eigene Berechnungen

Der für hohe Schulden weitgehend konstante marginale Erbschaftsnutzen in Höhe von $\xi$ bedeutet, daß der marginale Nutzen bei zunehmender Verschuldung nicht weiter ansteigt. Also reagiert die Optimalitätsbedingung (2.29) bei hoher Verschuldung kaum auf Veränderungen des Vermögens. Personen, deren Vermögen im Positiven wie im Negativen eine durch $\xi$ festgelegte Größe erreicht, gestalten daher ihr Konsumverhalten weitgehend unabhängig von Vermögensänderungen. Dagegen ändert sich der marginale Erbschaftsnutzen in der Nähe des Nullpunktes sehr schnell, so daß schon kleine Veränderungen des Vermögens in der Nähe des Nullpunktes einen großen Einfluß auf die Optimalitätsbedingungen haben. Personen in diesem Bereich reagieren also sehr stark auf Veränderungen ihres Vermögens.

Ob diese Eigenschaft des Erbschaftsnutzens (2.33) auf mikroökonomischer Ebene zu bestätigen ist, wäre eine interessante empirische Frage. Weil mir entsprechende Daten nicht zur Verfügung stehen, habe ich diese Frage auf mikroökonomischer Ebene nicht untersucht, dafür habe ich auf einer höher aggregierten Ebene, bei der Kalibrierung des Simulationsmodells im Abschnitt 3.6, verschiedene Erbschaftsnutzenfunktionen miteinander verglichen und den Erbschaftsnutzen (2.33) abgesichert: Sowohl Funktionen, deren marginaler Nutzen mit zunehmender Verschuldung weiter steigt, als auch Funktionen, deren marginaler Nut- 
zen mit zunehmender Verschuldung wieder abnimmt, ergeben eine schlechtere Anpassung an die Daten als die Erbschaftsnutzenfunktion (2.33). Eine lineare Erbschaftsnutzenfunktion, die dem Modell von Hurd (1989) zugrunde liegt, liefert ebenfalls eine deutlich schlechtere Anpassung, also nutzt das Erbschaftsnutzenmodell die zusätzliche Flexibilität der nichtlinearen Erbschaftsnutzenfunktion (2.33) aus.

Beispiel 1: Flexibles Konsumprofil

Das Vermögen einer Person am Anfang ihres Erwerbslebens ist gering und das Einkommen dieser Person nimmt einen normalen Verlauf. Welchen Verlauf nimmt das Konsumprofil?

Abbildung 2.7: Konsumprofile im Erbschaftsnutzenmodell und im Lebenszyklusmodell bei unsicherer Lebenserwartung ohne Erbschaftsmotiv.

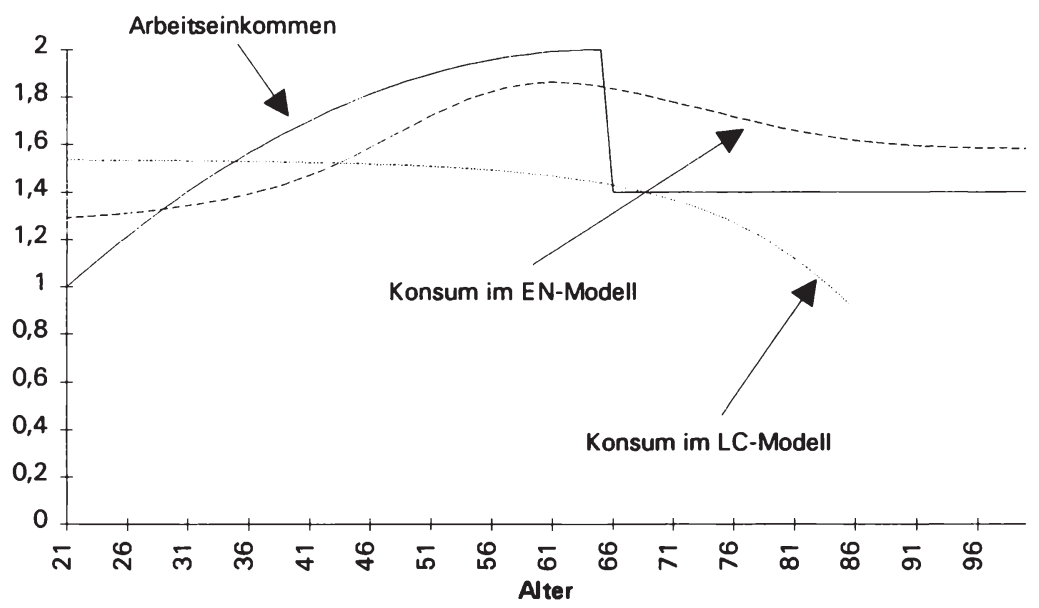

Anmerkungen:EN-Modell steht für das Erbschaftsnutzenmodell.

LC-Modell steht für das Lebenszyklusmodell bei unsicherer Lebenserwartung ohne Erbschaftsmotiv.

Quelle: $\quad$ Eigene Berechnungen 
Abbildung 2.7 verdeutlicht die im Vergleich zum klassischen Lebenszyklusmodell größere Flexibilität des Konsumprofils im Erbschaftsnutzenmodell für eine Person, die mit 21 Jahren ohne Anfangsvermögen ihr Erwerbsleben beginnt. Der Anfangslohn der Person beträgt $w_{21}=1$ Einheit. Während des Erwerbslebens steigt der Arbeitslohn mit dem Alter entlang einer quadratischen Funktion an und erreicht mit 65 Jahren sein Maximum von $w_{65}=2$. Im Alter von 66 Jahren tritt die Person in den Ruhestand und bekommt für den Rest ihres Lebens eine Rente in Höhe von $70 \%$ ihres letzten Arbeitslohnes, also $w_{R}=1,4$. Die folgenden Abbildungen zeigen die Profile vom 21. bis zum 100. Lebensjahr. ${ }^{22}$

Am Anfang des Erwerbslebens ist der marginale Erbschaftsnutzen aus zusätzlichem Vermögen hoch und daher impliziert die Eulergleichung (2.29), daß der marginale Konsumnutzen in Periode $t$ deutlich kleiner ist als der marginale Konsumnutzen in Periode $t-1$, also steigt das Konsumprofil schnell. Mit zunehmendem Vermögen wird die Steigung des Konsumprofils so lange flacher, bis der marginale Vermögensnutzen gegenüber der Diskontierung mit der Hazard-Rate $q$ unbedeutend wird, wodurch das Konsumprofil seine Steigung umkehrt und fällt. Wenn die Rente nicht ausreicht, um den Konsum im Ruhestand zu finanzieren, nimmt das Vermögen wieder ab, wodurch der marginale Erbschaftsnutzen wieder größer und die Steigung des Konsumprofils erneut flacher wird. Dieser Effekt wird verstärkt durch die im Alter zunehmende Sterbewahrscheinlichkeit, die zu steigenden Hazard-Raten $q$ führt. Das Beispiel zeigt, daß Konsumprofile im Erbschaftsnutzenmodell mehrere Wendepunkte haben können.

Im Vergleich zum klassischen Lebenszyklusmodell mit unsicherer Lebenserwartung, dessen Konsumprofil bis jenseits des 50 . Lebensjahres wegen der geringen Sterbewahrscheinlichkeiten praktisch konstant ist, verringert der Erbschaftsnutzen die MRSC: Eine zusätzliche Konsumeinheit in Periode $t$ erfordert im Erbschaftsnutzenmodell bei konstantem Nutzen einen geringeren Konsumverzicht in Periode $t-1$, denn durch den Konsumverzicht steigt das zu vererbende Vermögen und damit der Lebensnutzen. Weil das Vermögen am Anfang des Arbeitslebens der in Abbildung 2.7 gezeigten Person gering ist, ist der marginale Erbschaftsnutzen zusätzlicher Vermögenseinheiten so hoch, daß die niedrige Sterbewahrscheinlichkeit am Lebensanfang nicht ausreicht, um den marginalen Erbschaftsnutzen und die MRSA unbedeutend zu machen.

22 Bei der Berechnung der optimalen Profile werden auch die Alter 101 bis 120 berücksichtigt, denn erst mit 120 Jahren erreicht die Überlebenswahrscheinlichkeit den Wert null. Weil es aber nur sehr wenige Personen gibt, die ein Alter von 100 Jahren erreichen, beschränke ich die Abbildungen auf den relevanten Bereich. 
Abbildung 2.8: MRSA im Erbschaftsnutzenmodell

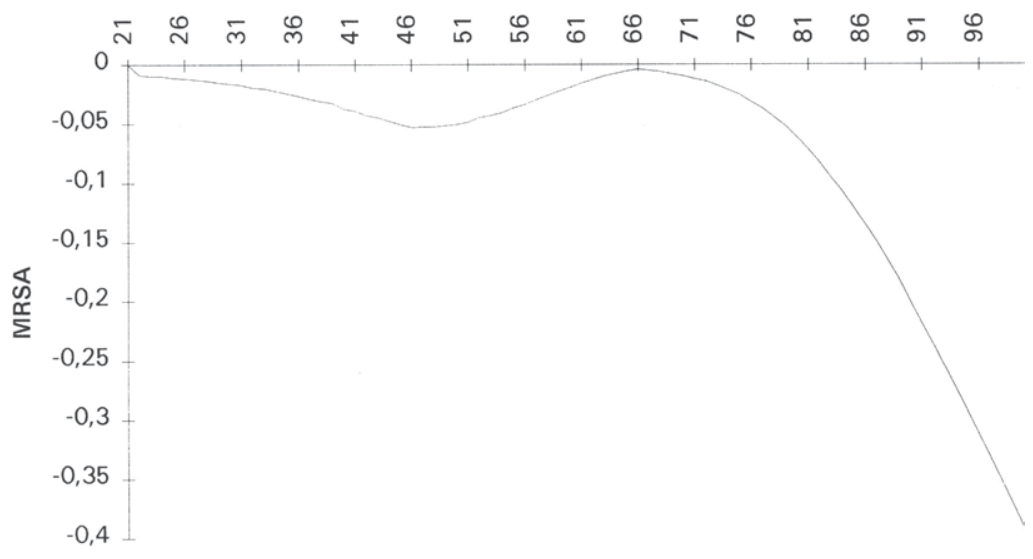

Alter

Quelle: Eigene Berechnungen

Abbildung 2.8 zeigt mit der MRSA die entscheidende Größe des Erbschaftsnutzenmodells für die selbe Person, die in Abbildung 2.7 dargestellt ist. Im ersten Drittel des Lebens ist das Vermögen gering und der Betrag der MRSA klein, aber deutlich von null verschieden. Durch den ab dem 35. Lebensjahr schnell steigenden Konsum (siehe Abbildung 2.7) wird der marginale Konsumnutzen kleiner und dieser Effekt führt zu der bis zum 50. Lebensjahr betragsmäßig ansteigenden MRSA. Bis zum 60. Lebensjahr steigt der Konsum zwar weiter an, doch ab dem 50. Lebensjahr dominiert das zunehmende Vermögen die Entwicklung der MRSA. Der marginale Erbschaftsnutzen nimmt in diesem Lebensabschnitt schnell ab. Nach der Verrentung geht der Konsum nur langsam zurück, also muß mit einem Teil des Vermögens der Konsum finanziert werden. Das sinkende Vermögen und die mit dem zunehmenden Alter steigende Sterbewahrscheinlichkeit erhöhen die MRSA nach der Verrentung kontinuierlich.

\section{Beispiel 2: Arbeitseinkommensprofil}

Ein wesentliches Ergebnis der theoretischen Diskussion des Erbschaftsnutzenmodells ist der Zusammenhang zwischen intertemporaler Allokation des Konsums und dem Einkommensverlauf. Dieses Beispiel verdeutlicht das theoretische Er- 
gebnis, indem es zwei Personen vergleicht, deren Arbeitseinkommen bei gleichem Barwert einen unterschiedlichen Verlauf nimmt. Die erste Person ist aus dem vorhergehenden Beispiel schon bekannt: Sie hat ein ansteigendes Arbeitseinkommen. Die zweite Person hat ein konstantes Arbeitseinkommen, dessen Barwert im 21. Lebensjahr dem Barwert des steigenden Arbeitseinkommensprofils der ersten Person gleicht. Abbildung 2.9 zeigt den Verlauf der Konsumprofile.

Abbildung 2.9: Konsumprofile im Erbschaftsnutzenmodell bei unterschiedlichen Arbeitseinkommensprofilen

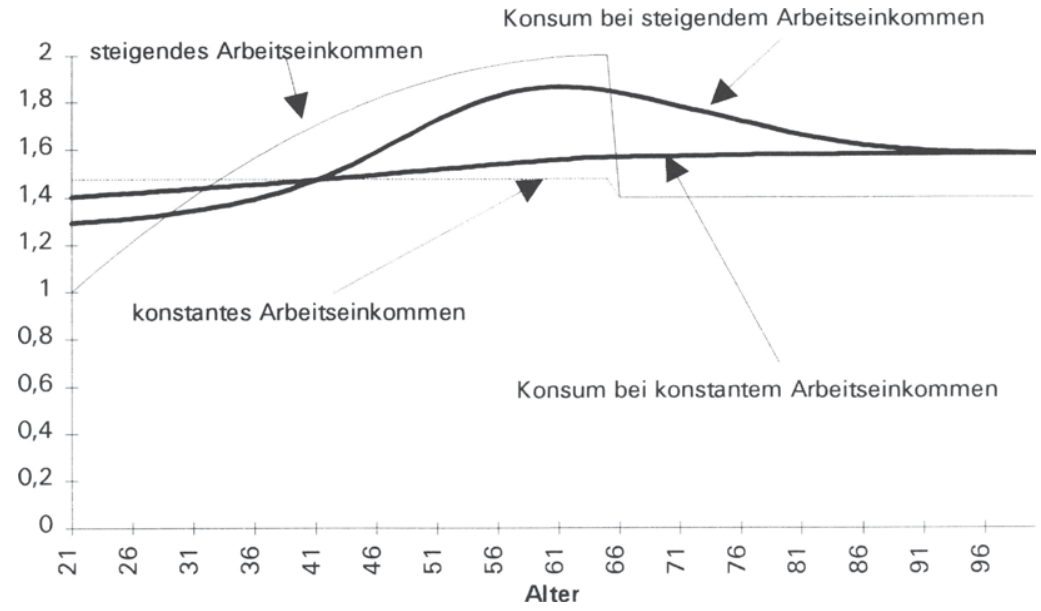

Quelle: Eigene Berechnungen

Der Unterschied zwischen den beiden Konsumprofilen in Abbildung 2.9 ist deutlich: Das optimale Konsumprofil der zweiten Person, die ein konstantes Arbeitseinkommen hat, steigt in dem abgebildeten Zeitraum kontinuierlich aber langsam an. Bis zum 41. Lebensjahr ist der Konsum geringer als das Arbeitseinkommen, so $\mathrm{da} B$ die Person mit konstantem Arbeitseinkommen schon in ihren ersten 20 Arbeitsjahren Vermögen akkumuliert. Dagegen verschuldet sich die Person mit steigendem Arbeitseinkommen am Anfang ihres Erwerbslebens: Bis zu ihrem 28. Lebensjahr übersteigt der Konsum das Arbeitseinkommen. Danach spart die erste Person einen Teil ihres Arbeitseinkommens, um erstens ihren Erben ein Vermögen zu hinterlassen und um zweitens im Ruhestand einen höheren Konsum aufrecht erhalten zu können. Das Konsumprofil der Person mit konstantem Arbeitseinkommen liegt ab dem 42. Lebensjahr unter dem Konsumprofil der Person mit 
steigendem Arbeitseinkommen. Dieser Nachteil wird durch das anfänglich höhere Vermögen jedoch mehr als ausgeglichen, denn der Lebensnutzen der Person mit konstantem Arbeitseinkommen ist um 7,5\% höher als der Lebensnutzen der Person mit steigendem Arbeitseinkommen. Dieser Nutzenunterschied wird durch die unterschiedlichen Vermögensprofile erklärt, die im Erbschaftsnutzenmodell einen direkten Einfluß auf die intertemporale Allokation des Konsums haben, im klassischen Lebenszyklusmodell aber nur indirekt über die Lebensbudgetrestriktion auf den Konsum einwirken.

Abbildung 2.10: Vermögensprofile im Erbschaftsnutzenmodell bei unterschiedlichen Arbeitseinkommensprofilen

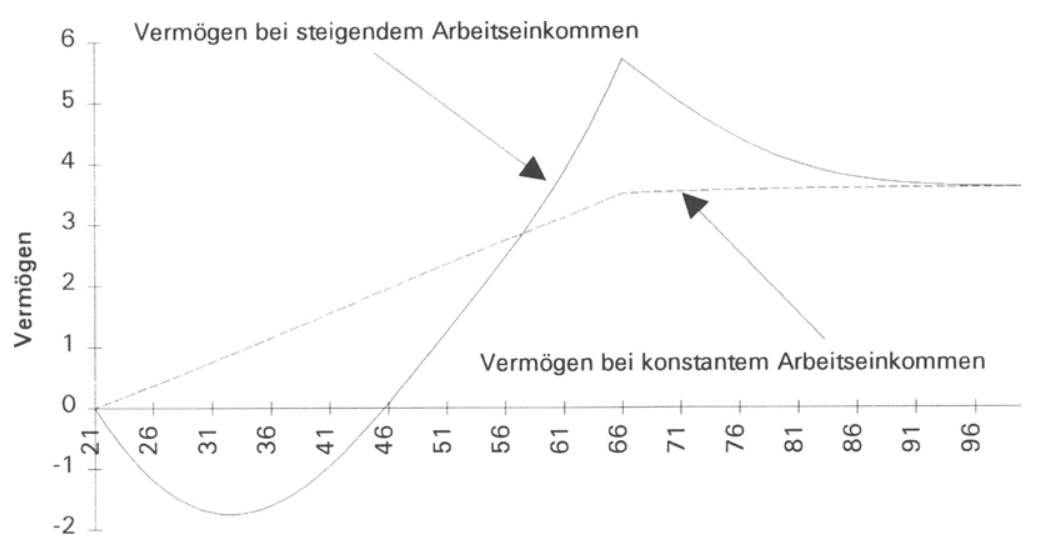

Alter

Quelle: Eigene Berechnungen

Abbildung 2.10 zeigt deutlich, daß die Person mit dem konstanten Arbeitseinkommen bis zum 57. Lebensjahr ein höheres Vermögen als die Person mit steigendem Arbeitseinkommen hat. Dieses höhere Vermögen hat zur Folge, daß die MRSA für die Person mit konstantem Arbeitseinkommen relativ kleiner ist als die MRSA der Person mit steigendem Arbeitseinkommen - damit erklärt sich das stärker geglättete Konsumprofil der Person mit konstantem Arbeitseinkommen. 
Beispiel 3: Geringes Arbeitseinkommen

Das dritte Beispiel verdeutlicht, wie die Höhe des Einkommens das Konsum- und Vermögensprofil beeinflußt.

Abbildung 2.11: Konsumprofile im Erbschaftsnutzenmodell bei unterschiedlicher Arbeitseinkommenshöhe

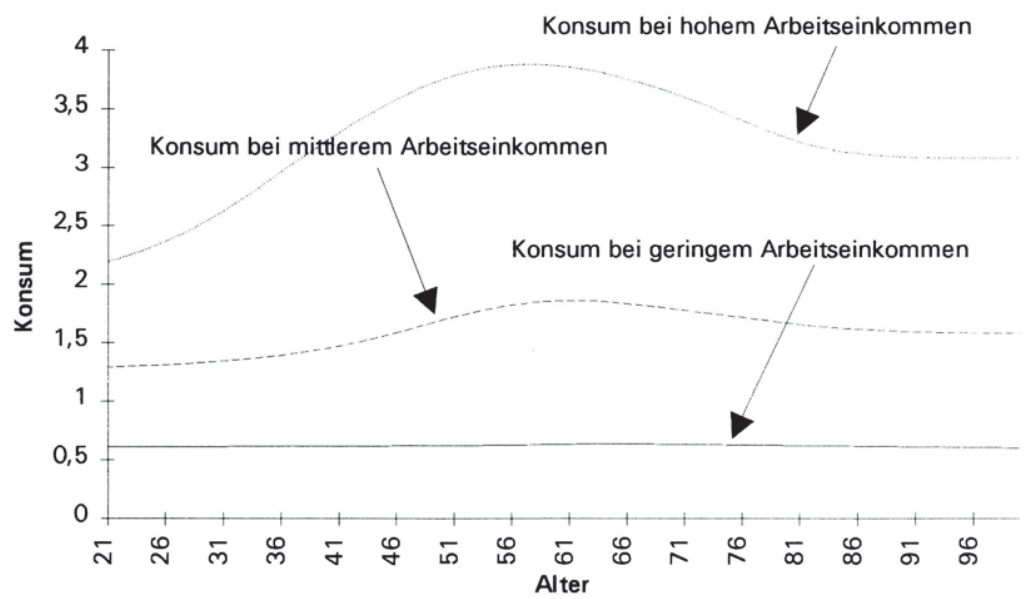

Quelle: Eigene Berechnungen

Erneut dient die Person aus Beispiel 1 als Referenzperson: Ihr Arbeitseinkommen verdoppelt sich mit dem Alter von $w_{21}=1$ auf $w_{65}=2$ entlang eines Polynoms zweiten Grades. Diese Person ist die Person mit mittlerem Arbeitseinkommen. Daneben gibt es zwei weitere Personen: eine mit niedrigem Arbeitseinkommen und eine mit hohem Arbeitseinkommen. Bei allen Personen entspricht der Verlauf des Arbeitseinkommens einem Polynom zweiten Grades. Die Person niedrigen Arbeitseinkommens beginnt ihr Arbeitsleben mit $w_{21}=0,42$ und die Person hohen Arbeitseinkommens erhält als Anfangseinkommen $W_{21}=2$. Weil der Verlauf der Einkommensprofile, der nach Beispiel 2 einen großen Einfluß auf die intertemporale Allokation des Konsums hat, identisch ist, isoliert dieses Beispiel den Einfluß der Einkommenshöhe.

Ein niedriges Arbeitseinkommen führt zu einem niedrigen Konsumniveau, also ist der marginale Konsumnutzen hoch und die MRSA klein. Die Theorie, Gleichung (2.32), impliziert, daß sich das Erbschaftsnutzenmodell um so mehr dem klassi- 
schen Lebenszyklusmodell annähert, je kleiner die MRSA ist. Genau diese Aussage bestätigt Abbildung 2.11: Das Konsumprofil der Person geringen Einkommens ist im wesentlichen flach und damit den Konsumprofilen des klassischen Lebenszyklusmodells relativ ähnlich. Die Person mit hohem Arbeitseinkommen kann am Anfang ihres Erwerbslebens relativ leichter auf Konsumeinheiten zugunsten des Vermögens verzichten, ihre MRSA ist anfänglich hoch und deswegen unterscheidet sich das Konsumprofil der reichen Person deutlich von Konsumprofilen des klassischen Lebenszyklusmodells.

Abbildung 2.12: Vermögensprofile im Erbschaftsnutzenmodell bei unterschiedlicher Arbeitseinkommenshöhe

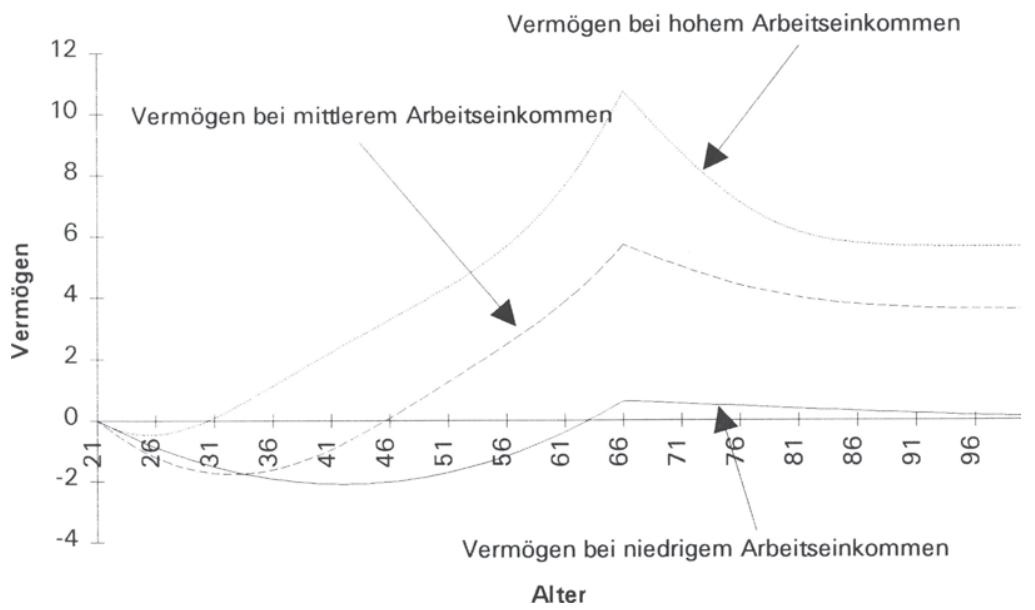

Quelle: Eigene Berechnungen

Das Vermögensprofil der Person geringen Einkommens ist fast während des gesamten Erwerbslebens negativ: Erst ab dem 43. Lebensjahr spart die Person niedrigen Einkommens, um ihre Schulden zu reduzieren. Im Jahr der Verrentung erreicht das Vermögen sein Maximum mit einem positiven Wert, um dann bis zum 100. Lebensjahr leicht abzunehmen, jedoch ohne negativ zu werden. Eine nennenswert Erbschaft leistet die Person mit geringem Arbeitseinkommen nicht. Völlig anders ist die Situation bei der Person mit mittlerem Arbeitseinkommen, denn sie hinterläßt ihren Nachkommen beim Ableben nach dem 80. Lebensjahr fast vier Geldeinheiten, also das Doppelte des zuletzt bezogenen Arbeitseinkommens. Auch in jungen Jahren unterscheidet sich der Vermögensverlauf der Person 
mittleren Arbeitseinkommens: Bis zum 33. Lebensjahr nimmt sie Schulden auf, danach steigt das Vermögen bis zur Verrentung an. Nach der Verrentung konsumiert die Person mittleren Arbeitseinkommens anfänglich mehr als ihre Rente, wie das abnehmende Vermögen zeigt. Mit zunehmendem Alter geht der Konsum jedoch zurück und nach dem 85 . Lebensjahr wird kaum noch entspart. Bei der Person mit hohem Arbeitseinkommen ist die Verschuldung am Anfang des Erwerbslebens noch geringer, dafür ist der Vermögensabbau in den ersten Jahren nach der Verrentung noch deutlicher als bei der Person mit mittlerem Arbeitseinkommen. Das ab dem 80. Lebensjahr zu vererbende Vermögen erreicht mit fast sechs Geldeinheiten aber nicht das Doppelte des zuletzt bezogenen Arbeitseinkommens von vier Geldeinheiten, denn der Erbschaftsnutzen ist durch abnehmenden Grenznutzen gekennzeichnet. Aus diesem Grund vererbt die Person mit mittlerem Arbeitseinkommen ein verhältnismäßig größeres Vermögen. Bei der Person mit niedrigem Arbeitseinkommen ist der marginale Erbschaftsnutzen im Vergleich zu den anderen Personen am höchsten, doch nun dominiert der sehr hohe marginale Konsumnutzen das Verhalten: Die Person mit niedrigem Einkommen kann sich Konsumverzicht schlicht nicht leisten.

\subsubsection{Kritische Würdigung}

Das Erbschaftsnutzenmodell kann leicht als Vermögensnutzenmodell interpretiert werden, indem man argumentiert, daß das Vermögen nicht nur durch das Erbschaftsmotiv, sondern auch durch andere Motive Nutzen stiftet. Angelegt ist dieser Gedanke schon in der klassischen Literatur: Keynes zählt in der General Theory acht Hauptmotive auf, mit denen er das Sparverhalten der privaten Haushalte erklärt:

“(i) To build up a reserve against unforeseen contingencies;

(ii) To provide for an anticipated future relation between the income and the needs of the individual or his family different from that which exists in the present, as, for example, in relation to old age, family education, or the maintenance of dependents;

(iii) To enjoy interest and appreciation, i.e. because a larger real consumption at a later date is preferred to a smaller immediate consumption;

(iv) To enjoy a gradually increasing expenditure, since it gratifies a common instinct to look forward to a gradually improving standard of life rather than the contrary, even though the capacity for enjoyment may be diminishing;

(v) To enjoy a sense of independence and the power to do things, though without a clear idea or definite intention of specific action; 
(vi) To secure a masse de manovre to carry out speculative or business projects;

(vii) To bequeath a fortune;

(viii) To satisfy pure miserliness, i.e. unreasonable but insistent inhibitions against acts of expenditure as such." (Keynes, 1935, pp. 107-108)

Keynes bringt die acht Sparmotive unter der Kapitelüberschrift "The Propensity to Consume: II. The Subjective Factors", nachdem er den Lohn, die Differenz zwischen Brutto- und Nettolohn, die Zeitpräferenz, die Fiskalpolitik und Änderungen in den Erwartungen unter der Überschrift objective factors diskutiert hat. Das klassische Lebenszyklusmodell bildet die objektiven Faktoren weitgehend $\mathrm{ab}$, berücksichtigt die subjektiven Faktoren jedoch kaum. Ein eigenständiger Vermögensnutzen kann als ein Versuch gesehen werden, diese subjektiven Faktoren in praktikabler Weise in den Rahmen des Lebenszyklusmodells zu integrieren.

Die bisherige Diskussion des Erbschaftsnutzenmodells klärte hauptsächlich die Mechanik, mit der die Konsum- und Vermögensprofile erzeugt werden. Abschließend bringe ich einige Überlegungen zur Plausibilität des Erbschaftsnutzenmodells. Nach Abschnitt 2.1.2 ist das keine leichte Aufgabe, denn die Ergebnisse wichtiger empirischer Arbeiten liefern kein klares Bild beispielsweise zum Vermögensverlauf in Alter, und sind daher heftig umstritten. Aus mikroökonomischer Sicht auf der Ebene der Haushalte kann man immerhin festhalten, daß sich das Konsum- und Sparverhalten mit den Einkommensklassen verändert. Belege liefern beispielsweise die Arbeiten von Bosworth et al. (1991), Börsch-Supan (1994) und Allesie et al. (1995), die für die unteren Einkommensklassen für kein Alter ein Vermögen finden, das über die Konsumausgaben einiger Monate hinausgeht. Das Erbschaftsnutzenmodell kann diese Beobachtung gut erklären, denn die Person geringen Einkommens in Abbildung 2.12 hat ebenfalls zu keinem Zeitpunkt ein bedeutendes Vermögen - allerdings hat sie nennenswerte Schulden. Dies ist eher unrealistisch, denn gerade Personen mit geringem Einkommen sind in der Realität liquiditätsbeschränkt. Das Erbschaftsnutzenmodell kennt in der hier entwickelten Version keine Liquiditätsbeschränkung - diese Annahme ist zweifellos problematisch. Wenn ich hier von Liquiditätsbeschränkungen abstrahiere, dann liegt das in erster Linie an den numerischen Schwierigkeiten, die durch die Liquiditätsbeschränkungen verursacht werden. Zur Verteidigung kann ich immerhin anführen, da $B$ ich das Erbschaftsnutzenmodell im allgemeinen Gleichgewicht verwende, um das Verhalten des durch Aggregation aller Haushalte entstehenden "durchschnittlichen“ Haushalts je Kohorte zu beschreiben. Die Liquiditätsbeschränkungen für die unteren Einkommensklassen werden durch die Aggregation natürlich nicht aufgehoben, für den „durchschnittlichen“ Haushalt verliert sie aber doch an Bedeutung. 
Empirische Konsumprofile sind ähnlich umstritten wie empirische Vermögensprofile. Auf der Basis der Einkommens- und Verbrauchsstichprobe 1983 finden Börsch-Supan und Stahl (1991) eine klare Veränderung des Konsums mit dem Alter. Zumindest nach der Verrentung nimmt der Konsum mit zunehmendem Alter ab. Börsch-Supan und Stahl erklären diese Beobachtung mit der abnehmenden körperlichen Leistungsfähigkeit alternder Menschen, wodurch die Konsummöglichkeiten zurückgehen. Das Erbschaftsnutzenmodell bringt in den Beispielen für die Person hohen Einkommens ebenfalls ein fallendes Konsumprofil nach dem 55. Lebensjahr, also kann das Erbschaftsnutzenmodell die Beobachtung von Börsch-Supan und Stahl erklären, ohne Konsumrestriktionen bemühen zu müssen.

Das hier entwickelte Erbschaftsnutzenmodell geht auf das Modell von Hurd (1989) zurück. Hurd verwendet allerdings einen konstanten marginalen Erbschaftsnutzen - dadurch ist sein Modell stark eingeschränkt. Tatsächlich ist der variable marginale Erbschaftsnutzen ganz entscheidend für die vielfältigen Möglichkeiten des Erbschaftsnutzenmodells, denn der marginale Erbschaftsnutzen bestimmt die MRSA, die wiederum den Unterschied zum klassischen Lebenszyklusmodell bestimmt.

Abschließend halte ich fest, daß das Konsum- und Sparverhalten der Haushalte mit dem Erbschaftsnutzenmodell flexibler beschrieben werden kann als mit dem klassischen Lebenszyklusmodell und seiner Erweiterung um ein Erbschaftsmotiv bei sicherer Lebenserwartung. Die Simulationen von Auerbach et al. (1989), Raffelhüschen und Kitterer (1990) sowie Buslei und Kraus (1996) gehen von der Erweiterung des klassischen Lebenszyklusmodells um einen Erbschaftsnutzen bei sicherer Lebenserwartung aus und lassen sich nicht zufriedenstellend kalibrieren: Problematisch sind das hohe Gewicht des Erbschaftsnutzens und die negative Zeitpräferenzrate, die Auerbach et al. für Deutschland annehmen müssen. Weil das Erbschaftsnutzenmodell die unsichere Lebenserwartung realistischer modelliert, sollte sich eine Simulation mit dem Erbschaftsnutzenmodell als Haushaltsmodell leichter kalibrieren lassen. 


\section{Das Simulationsmodell}

Die Diskussion des Erbschaftsnutzenmodells im zweiten Kapitel hat verdeutlicht, warum ein Erbschaftsmotiv bei unsicherer Lebenserwartung das Lebenszyklusmodell mit Erbschaftsmotiv bei sicherem Planungshorizont wesentlich erweitert. In diesem dritten Kapitel füge ich das Erbschaftsnutzenmodell mit der Bevölkerungsprognose, dem Produktionssektor und der Rentenversicherung zu einem allgemeinen Gleichgewichtsmodell zusammen, dessen Simulation im vierten Kapitel Reformen der Rentenversicherung und deren makroökonomische Auswirkungen untersuchen soll.

\subsection{Der Haushaltssektor}

Im zweiten Kapitel habe ich das Erbschaftsnutzenmodell aus der Sicht einer Person dargestellt, die bei gegebenem Einkommen ihren erwarteten Lebensnutzen aus dem Konsum und der hinterlassenen Erbschaft am a priori nicht bekannten Lebensende maximiert. Dieser Abschnitt erweitert das Erbschaftsnutzenmodell in zwei Richtungen: Das Erbschaftsnutzenmodell wird explizit als Haushaltsmodell formuliert und das Einkommen wird durch die nutzenmaximierende Wahl der Freizeit endogenisiert.

\subsubsection{Das Erbschaftsnutzenmodell als Haushaltsmodell}

Der Übergang von Personen auf Haushalte als Entscheidungsträger wirft eine Reihe von Fragen auf, die mit der Veränderung der Haushaltszusammensetzung über die Zeit zusammenhängen, zum Beispiel Heirat, Geburt von Kindern, Auszug von Kindern und Tod von Haushaltsmitgliedern. Um diese Veränderungen im Lebenszyklus eines Haushalts im Erbschaftsnutzenmodell zu berücksichtigen, nehme ich an, daß die einzelnen Haushaltsmitglieder ihre individuellen Präferenzen zurückstellen und nur am Lebensnutzen des gesamten Haushalts interessiert sind. Wie der Konsum, das Vermögen und das Einkommen innerhalb eines Haushalts aufgeteilt werden, ist eine Frage, die ich hier nicht behandeln werde. Eine zweite wesentliche Vereinfachung ergibt sich aus dem stilisierten Lebenszyklus, den ich den Haushalten unterstelle: Alle Haushalte bestehen nach der Kinderphase aus zwei gleichaltrigen Personen, die zur selben Zeit sterben. Folglich ist die Sterbewahrscheinlichkeit dieser stilisierten Haushalte und die Sterbewahrscheinlichkeit der Personen identisch. In Abschnitt 3.2.2 schildere ich den unterstellten Lebenszyklus ausführlich und diskutiere seine Problematik. 
Auerbach et al. (1989) gehen von Personen auf Haushalte über, indem sie in ihrer Simulation den Nutzen jeder Periode mit der Haushaltsgröße der entsprechenden Periode gewichten, also hat der Periodennutzen ein hohes Gewicht, wenn der Haushalt groß ist. Den Vorschlag von Auerbach et al. aufgreifend, schreibe ich mit der Variablen $h_{t}$ als Haushaltsgröße im Jahr $t$ den erwarteten Lebensnutzen des Haushalts als:

$$
E_{\tau}(U)=\sum_{t=\tau}^{T}(1+\rho)^{\tau-t} \cdot h_{t} \cdot\left[s_{t}^{\tau} u\left(c_{t}\right)+m_{t}^{\tau} v\left(a_{t}\right)\right]
$$

Diese Modellierung der Haushaltsgröße impliziert eine Äquivalenzskala $z$, die sich aus der folgenden Gleichung ergibt:

$$
h \cdot u(c)=u\left(\frac{c}{z}\right)
$$

Für den Fall der isoelastischen Nutzenfunktion mit $\gamma=3$ ist die Äquivalenzskala $z$ die Quadratwurzel aus $h$. Ein Zwei-Personen-Haushalt hat demnach aus 1,41 Konsumeinheiten den gleichen Nutzen wie ein Ein-Personen-Haushalt aus einer Konsumeinheit; für einen Drei-Personen-Haushalt beträgt die Äquivalenzskala 1,73 .

\subsubsection{Endogenes Arbeitsangebot}

Bisher bestimmen die Haushalte im Erbschaftsnutzenmodell über ihr Konsumund Vermögensprofil bei exogen gegebenem Arbeitseinkommen. Wenn die Beiträge zur umlagefinanzierten Rentenversicherung als Prozentsatz des Bruttolohns erhoben werden, dann vergrößert der Alterungsproze $B$ wegen steigender Beitragssätze die Schere zwischen Brutto- und Nettolohn. In der Einleitung habe ich die Diskussion pro und contra Umstieg auf eine kapitalgedeckte Alterssicherung als eine Motivation für meine Arbeit angeführt. Ein wesentlicher Aspekt dieser Diskussion ist die Verzerrung des Arbeitsangebotes, die durch den Beitragssatz zur Rentenversicherung ausgelöst werden kann. Solange die eingezahlten Beiträge zu einer versicherungsmathematisch fairen Rente führen, ergibt sich keine Verzerrung des Arbeitsangebotes, aber je größer der Unterschied zwischen den eingezahlten Beiträgen und der versicherungsmathematisch fairen Rente ist, desto mehr wird das Arbeitsangebot verzerrt. Da die umlagefinanzierte Rentenversicherung einem Generationenvertrag entspricht, wird der Alterungsprozeß notwendig den Unterschied zwischen den eingezahlten Beiträgen und der versicherungsmathematisch fairen Rente vergrößern. Im schlimmsten Fall wäre ein Teufelskreis vorstellbar: Höhere Beitragssätze veranlassen die Leute, ihr Arbeitsangebot zu- 
rückzunehmen oder auf die Schattenwirtschaft auszuweichen. Dadurch schrumpft die Basis der Beitragszahler, also müssen die verbleibenden Beitragszahler noch höhere Beiträge entrichten, um die konstanten Beitragsverpflichtungen zu decken. Letztlich würde dieser Teufelskreis zum Ende des Generationenvertrags führen.

Um die Wechselwirkungen zwischen Arbeitsangebot und umlagefinanzierter Rentenversicherung untersuchen zu können, erweitere ich den erwarteten Lebensnutzen um den Nutzen der Freizeit, die sich aus der Differenz zwischen der auf eine Einheit pro Person normierten maximal verfügbaren Zeit und der Arbeitszeit ergibt. Im Gegensatz zum Konsumnutzen gewichte ich den Freizeitnutzen nicht mit der Haushaltsgröße, sondern mit der Anzahl der Haushaltsmitglieder im Erwerbsalter, denn nur für diese Personen ist die Arbeits- bzw. Freizeit eine Entscheidungsvariable. Die Erwerbspersonen $b$ bestehen aus den Haushaltsmitgliedern im Alter von $\tau$ (Berufseinstieg) bis $T R$ (Verrentung); beide Alter sind exogen gegeben. Nach der Verrentung beträgt die Freizeit die maximal verfügbare Zeit. Den Nutzen, den die erwachsenen Haushaltsmitglieder aus ihrer gemeinsamen Freizeit haben, beschreibt die Funktion $f$. Die gemeinsame Freizeit $/$ ist die Summe der Freizeit der erwachsenen Haushaltsmitglieder. Nach der Verrentung setze ich $b=h$, weil die Kinder bis dahin in der Regel (und dem hier angenommenen Lebenszyklus) den elterlichen Haushalt verlassen haben. Der Nutzen der Freizeit nach der Verrentung ist für die Berechnung des Lebensnutzens wichtig, nicht aber für die Optimierung, denn die Freizeit ist im Ruhestand exogen gegeben.

Analog zur Erweiterung des Konsumnutzens um den Erbschaftsnutzen füge ich den Freizeitnutzen additiv-separabel dem Lebensnutzen hinzu. Die unterstellte Separabilität zwischen Konsum und Freizeit (und auch dem Vermögen) schränkt die Substitutionsbeziehungen zwischen Konsum und Freizeit ein - ein Punkt, auf den ich gleich zurückkommen werde.

$$
E(U)=\sum_{t=\tau}^{T}(1+\rho)^{\tau-t} \cdot h_{t} \cdot\left[s_{t}{ }^{\tau} u\left(c_{t}\right)+m_{t}{ }^{\tau} v\left(a_{t}\right)\right]+\sum_{t=\tau}^{T}(1+\rho)^{\tau-t} \cdot b_{t} \cdot s_{t}{ }^{\tau} \cdot f\left(I_{t}\right)
$$

Das Arbeitsangebot des Haushalts beträgt $(b-l)$ Zeiteinheiten und das Arbeitseinkommen des Haushalts beläuft sich auf $(b-l) \cdot w$ Geldeinheiten. Im Ruhestand erhält der Haushalt eine Rente $w^{R}$ von der Rentenversicherung. Die Budgetrestriktion besteht aus zwei Gleichungen, die das Erwerbsleben und den Ruhestand beschreiben, wobei erb für die erhaltenen Erbschaften steht:

$$
\begin{array}{ll}
a_{t+1}=\left(1+r_{t}\right) a_{t}+\left(b_{t}-l_{t}\right) w_{t}-c_{t}+e r b_{t} & \text { für } t=\tau, \ldots, T^{R} \\
a_{t+1}=\left(1+r_{t}\right) a_{t}+w_{t}^{R}-c_{t}+e r b_{t} & \text { für } t=T^{R}+1, \ldots, T
\end{array}
$$


Nach der Verrentung muß der Haushalt auf jeden Fall aus dem Arbeitsmarkt ausscheiden. Davor, während des Erwerbslebens, kann der Haushalt freiwillig aus dem Arbeitsmarkt ausscheiden, wenn er die verfügbare Zeit vollständig für die Freizeit verwendet und $I=b$ wählt. Zusätzlich zur Budgetrestriktion (3.4) muß $0 \leq l \leq b$ gelten, denn die Freizeit kann weder negativ noch größer als die maximal verfügbare Zeit sein; in den Optimalitätsbedingungen setzt der Kuhn-Tucker Multiplikator $\mu$ diese Restriktion durch.

Für $t=\tau+1, \ldots, T$ beschreibt Gleichung (3.5) das optimale Konsum- und Vermögensprofil:

$$
\frac{\left(1+r_{t}\right)}{(1+\rho)} \frac{h_{t}}{h_{t-1}}\left(1-q_{t}\right) u^{\prime}\left(c_{t}\right)+\frac{1}{(1+\rho)} \frac{h_{t}}{h_{t-1}} q_{t} v^{\prime}\left(a_{t}\right)=u^{\prime}\left(c_{t-1}\right)
$$

Wegen $q_{T}=1$ ist in Gleichung (3.5) auch die Bedingung für das optimale Endvermögen enthalten.

Die optimale Freizeit (das Arbeitsangebot) in den relevanten Perioden $t=\tau, \ldots, T R$ bestimmt die folgende Gleichung:

$$
f^{\prime}\left(I_{t}\right)=\frac{h_{t}}{b_{t}} \cdot w_{t} \cdot u^{\prime}\left(c_{t}\right)+\tilde{\mu}_{t} \quad \text { für } t=\tau_{,} \ldots, T^{R}
$$

Die Optimalitätsbedingung (3.5) ist wegen der Separabilitätsannahme unabhängig vom Freizeitnutzen. Das Freizeitprofil hängt ab vom Konsum- und Lohnprofil (sowie dem Kuhn-Tucker Multiplikator, der nur dann von null verschieden ist, wenn die Restriktion greift). Steigender Lohn erhöht ceteris paribus den marginalen Freizeitnutzen: Die Haushalte schränken ihre Freizeit ein, um ein hohes Einkommen zu erzielen. Dagegen besteht ceteris paribus zwischen dem marginalen Konsum- und Freizeitnutzen eine positive Abhängigkeit: Steigender Konsum erhöht die Freizeit und senkt die Arbeitszeit. Bei der Diskussion des Erbschaftsnutzenmodells in Kapitel 2 habe ich die Optimalitätsbedingung (3.5) durch die marginalen Raten der Substitution MRSA und MRSC ausgedrückt. Die Bedingung für die nutzenmaximale Freizeit (3.6) kann ebenfalls durch eine marginale Rate der Substitution (hier zwischen Freizeit und Konsum) angegeben werden, wobei ich hier zur Vereinfachung von dem Kuhn-Tucker Multiplikator $\mu$ abstrahiere:

$$
M R S L_{t}=\frac{d c_{t}}{d l_{t}}=-\frac{b_{t}}{h_{t}} \frac{f^{\prime}\left(I_{t}\right)}{u^{\prime}\left(c_{t}\right)}=-w_{t}=-\left(1-\sigma_{t}\right) w_{t}^{\text {bruto }}
$$

Sinkt die Freizeit um eine Einheit, bleibt der Lebensnutzen unverändert wenn der Konsum um $w$ Einheiten steigt. Umgekehrt folgt, wenn $w$ steigt, dann muß man 
den Haushalten immer mehr Konsum bieten, um sie zu einem Freizeitverzicht zu bewegen. Aus Gleichung (3.7) wird auch die verzerrende Wirkung eines lohnbezogenen Beitragssatzes $\sigma$ deutlich: Bei steigendem Beitragssatz sinkt das Tauschverhältnis von Freizeit gegen Konsum. Durch die Separabilitätsannahme hat der Substitutionseffekt, also auch die MRSL, eine einfache Struktur. Der Gesamteffekt von Änderungen des Beitragssatzes $\sigma$ oder des Bruttolohnes wbrutto setzt sich aber aus dem Substitutions- und Einkommenseffekt zusammen, so da $B$ von dem Vorzeichen des Substitutionseffekts nicht auf das Vorzeichen des Gesamteffekts geschlossen werden kann. Also beschränkt die Separabilität im allgemeinen nicht die Richtung des Gesamteffekts, vielmehr hängt dieser wesentlich von der Spezifikation der Nutzenfunktion ab. Die Separabilitätsannahme beschränkt die Substitutionseffekte, die hier nur von den beiden betrachteten Variablen abhängen.

\subsubsection{Spezifikation der Lebensnutzenfunktion}

Die Lebensnutzenfunktion (3.3) faßt den Konsum-, Erbschafts- und Freizeitnutzen zusammen, also müssen diese drei Funktionen für das Simulationsmodell spezifiziert werden. Wie bei den Beispielprofilen in Kapitel 2 wähle ich die in früheren Arbeiten - beispielsweise Cutler et al. (1990) sowie Börsch-Supan (1995c) - oft verwendete isoelastische Nutzenfunktion für die Konsumpräferenzen der Haushalte:

$$
u(c)=\frac{1}{1-\gamma} c^{1-\gamma} \quad \text { mit } \gamma>0
$$

Auch die auf allen reellen Zahlen definierte Erbschaftsnutzenfunktion mit positiv abnehmendem Grenznutzen habe ich mit Formel (2.33) schon vorgestellt:

$$
v(a)=-\xi \ln (1+\exp (-a)) \quad \text { mit } \xi>0
$$

Dagegen tritt der Freizeitnutzen an dieser Stelle neu hinzu. Empirische Lebenszyklusmodelle mit endogenem Arbeitsangebot verwenden für den Freizeitnutzen häufig eine logarithmische Funktion ${ }^{1}$, zum Beispiel die Arbeiten von Heckman und MaCurdy (1982) sowie Schnabel (1994). Ich orientiere mich an diesen Arbeiten und spezifiziere den Freizeitnutzen ebenfalls logarithmisch:

1 Die logarithmische Funktion ist ein Spezialfall der isoelastischen Funktion: Wenn $\gamma$ in Gleichung (3.7) gegen eins geht, dann ergibt sich im Grenzübergang die logarithmische Funktion. 


$$
f(I)=\eta \ln (I) \quad \text { mit } \quad \eta>0
$$

Mit diesen Spezifikationen hat die Lebensnutzenfunktion (3.3) vier exogene Parameter: die Zeitpräferenzrate $\rho, \gamma$ aus dem Konsumnutzen, $\xi$ aus dem Erbschaftsnutzen und $\eta$ aus dem Freizeitnutzen.

\subsection{Die Bevölkerungsprognose}

\subsubsection{Das Prognoseverfahren}

Die „Achte koordinierte Bevölkerungsvorausberechnung“ - siehe Sommer (1994) - schätzt die Entwicklung der Bevölkerung bis zum Jahr 2040 ein und ist die zur Zeit gültige Prognose des Statistischen Bundesamtes für Gesamtdeutschland. Die wichtigsten Annahmen dieser Vorausberechnung betreffen die drei wesentlichen Parameter der Bevölkerungsentwicklung: die Fertilität, die Mortalität und die Migration.

"Hinsichtlich der Geburtenhäufigkeit wurde für das frühere Bundesgebiet davon ausgegangen, daß sie - entsprechend einer nahezu zwei Jahrzehnten währenden Stagnation - auf dem derzeitigen Niveau verharrt. [...]

Bei der Sterblichkeit wurde - basierend auf dem bisherigen Trend - zunächst davon ausgegangen, daß sie weiter zurückgeht und die Lebenserwartung Neugeborener im früheren Bundesgebiet [...] bis zum Jahr 2000 um über 1,5 Jahre zunimmt und dann konstant bleibt. [...]

Zum Wanderungsverhalten der Ausländer wurden drei unterschiedliche Varianten vorgesehen, da hier die künftige Entwicklung besonders unsicher erscheint und sich auch in der Vergangenheit sehr starke Schwankungen ergaben. Ausgehend von den bis 1992 bekannten Wanderungsbewegungen wurde eine Abnahme der jährlichen Wanderungssalden auf 100000 (Variante 1), 200000 (Variante 2) bzw. 300000 (Variante 3) bis zum Jahr 2000 angenommen, und diese Werte wurden dann bis zum Ende des Vorausberechnungszeitraumes beibehalten." (Sommer, 1994, S. 497ff.)

Die Bevölkerungsprognose meines Simulationsmodells unterscheidet sich in einigen Punkten von den Annahmen des Statistischen Bundesamtes. Als Basis wähle ich die Bevölkerung in den alten Bundesländern im Jahr 1989, denn die demographische Struktur in den alten Bundesländern hat sich bis zur Wiedervereinigung 1990 relativ stetig entwickelt, während die vereinigungsbedingten Ost-West Wanderungen zu einer sprunghaften Veränderung führten; diese Veränderung hängt nicht mit dem Alterungsprozeß zusammen. Das Jahr vor der Wiederverei- 
nigung, 1989, ist daher ein sinnvolles Basisjahr. Die alten Bundesländer sind darüber hinaus eine geeignete Basis, weil das Simulationsmodell mit Daten aus der volkswirtschaftlichen Gesamtrechnung kalibriert werden soll und die entsprechenden Daten aus der ehemaligen DDR wenig mit den zukünftigen wirtschaftlichen Bedingungen gemein haben werden.

Die Fertilitätsraten für das Basisjahr 1989 entnehme ich dem Statistischen Jahrbuch 1992 (siehe Statistisches Bundesamt, 1992b). Bei der Simulation verschiedener Politikszenarien verwende ich die Werte von 1989 unverändert für alle Prognosejahre in Übereinstimmung mit der achten koordinierten Bevölkerungsprognose des Statistischen Bundesamtes. Um die Sensitivität des Simulationsmodells bezüglich der Fertilitätsraten einschätzen zu können, untersuche ich im vierten Kapitel in einem Szenario die Auswirkungen einer zukünftig wieder steigenden Fertilität.

Über die zukünftige Entwicklung der Mortalitätsraten gibt es deutlich unterschiedliche Auffassungen. Umstritten ist, wie schnell und wie lange die Mortalität weiter abnimmt, oder anders gesagt, wie schnell die durchschnittliche Lebenserwartung Neugeborener zunimmt. Tabelle 3.1 liefert mit dem Vergleich der Lebenserwartung in einigen ausgewählten Ländern eine Basis, die für die Fortschreibung der Lebenserwartung Anhaltspunkte liefert.

Tabelle 3.1: Durchschnittliche Lebenserwartung Neugeborener in Jahren

\begin{tabular}{|c|c|c|c|c|}
\hline Land & Jahr & Frauen & Männer & Mittel \\
\hline Frankreich & 1991 & 81,1 & 73,0 & 77,05 \\
\hline Japan & 1990 & 82,5 & 76,2 & 79,35 \\
\hline Schweden & 1991 & 80,5 & 75,0 & 77,75 \\
\hline Schweiz & $1990 / 91$ & 80,9 & 74,1 & 77,5 \\
\hline USA & 1990 & 78,8 & 72,0 & 75,4 \\
\hline Deutschland (West) & $1988 / 90$ & 79,0 & 72,6 & 75,8 \\
\hline
\end{tabular}

Quelle: Statistisches Bundesamt (1993)

Auffallend ist die vergleichsweise geringe Lebenserwartung in Deutschland: Gegenüber Japan ist sie um 3,5 Jahre geringer. Die Annahme des Statistischen Bundesamtes in der achten koordinierten Bevölkerungsvorausberechnung geht davon aus, daß sich die durchschnittliche Lebenserwartung Neugeborener bis zum Jahr 2000 um 1,5 Jahre erhöht und dann konstant bleibt, also die schon heute realisierten Werte in Japan und Schweden nicht erreicht. Börsch-Supan (1995a) nimmt 
dagegen an, daß die Lebenserwartung kontinuierlich weiter steigt und die gegenwärtigen japanischen Werte im Jahr 2010 annimmt. Ich wähle für meine Bevölkerungsprognose eine geringere Zunahme der Lebenserwartung als Börsch-Supan, gehe im Gegensatz zum Statistischen Bundesamt aber auch nach dem Jahr 2000 von weiter steigenden Werten aus. Erst ab dem Jahr 2050 setze ich die Sterblichkeit konstant. Vor dem Hintergrund zunehmender Kosten im Gesundheitswesen halte ich dieses vorsichtige Vorgehen für angemessen, denn der medizinische Fortschritt wird zukünftig kaum im bisherigen Ausmaß finanziert werden können. Diese Entwicklung ist die Begründung für die im Vergleich zu Börsch-Supans Arbeiten langsamere Zunahme der Lebenserwartung. In meiner Bevölkerungsprognose steigt die durchschnittliche Lebenserwartung Neugeborener bis zum Jahr 2000 auf 77,2 Jahre, erreicht in 2010 78,2 Jahre, in 2030 79,3 Jahre und in 2050 schließlich 80,3 Jahre. Um einen Eindruck von der Sensitivität des Simulationsmodells bezüglich der Mortalitätsentwicklung zu bekommen, hält ein Szenario im vierten Kapitel die Mortalitätsziffern nach dem Jahr 2000 konstant und zeichnet folglich die Annahmen der achten koordinierten Bevölkerungsvorausberechnung des Statistischen Bundesamtes nach.

Weil die Entwicklung der Migration die Bevölkerungsentwicklung stark beeinflußt und nur schwer vorherzusehen ist, greife ich die Idee der achten koordinierten Bevölkerungsprognose auf und simuliere im vierten Kapitel Szenarien mit jährlichen Migrationssalden von 200000 und 400000 Einwanderern. Um auch diese Annahme in den Kontext zu stellen, gebe ich die Annahmen einiger Prognosen wieder: Das Institut für Arbeitsmarkt und Berufsforschung, kurz IAB, geht für die alten Bundesländer von einem Wanderungssaldo von 224000 in Jahr 1995 aus, der bis zum Jahr 2000 auf 145000 fällt und im Jahr 2010 den dann langfristig konstanten Wert von 129000 Einwanderern erreicht (siehe Thon, 1991). Börsch-Supan (1995a, 1995b) orientiert sich an diesen Zahlen, nimmt jedoch nur $80 \%$ der Werte des IAB. Das Prognos-Institut untersucht in seinem Rentengutachten (siehe Verband Deutscher Rentenversicherungsträger, 1995) ähnlich wie das Statistische Bundesamt zwei Varianten: Die obere Variante geht für die alten Bundesländer von 339000 (1995), 256000 (2010) und schließlich 245000 (ab 2020) jährlichen Einwanderern aus. Wesentlich geringer sind die Migrationssalden der unteren Variante des Prognos-Institutes: $81000 \mathrm{im}$ Jahr 2000, $58000 \mathrm{im}$ Jahr 2010 auf $47000 \mathrm{ab}$ dem Jahr 2020. Meine Basisvariante mit 200000 Einwanderern pro Jahr ab dem Jahr 2000 ist folglich niedriger als die obere Variante des Prognos-Institutes, aber höher als die IAB-Prognose.

Die Migrationssalden eines Jahres setzen sich aus Frauen und Männern unterschiedlichen Alters zusammen, so da $B$ der Saldo eines Jahres auf Frauen und Männer verschiedenen Alters aufgeteilt werden muß. Ich nehme an, daß sich die Geschlechts- und Alterszusammensetzung der Migranten in den Prognosejahren nicht verändert und teile die Salden mit den entsprechenden Zahlen von 1989 
auf. ${ }^{2}$ Weiterhin nehme ich an, daß sich die Migranten im Jahr nach ihrer Einwanderung der einheimischen Bevölkerung angleichen, also die gleichen Fertilitätsund Mortalitätsraten haben. Diese Annahme ist eine starke Vereinfachung, tatsächlich geschieht die Angleichung über einen längeren Zeitraum. Meine vereinfachende Annahme schwächt den positiven Effekt der Einwanderung in zweifacher Hinsicht ab: Erstens wird die höhere Fertilität unterschätzt und zweitens die geringere Lebenserwartung überschätzt. Wenn ich die Auswirkungen höherer Migrationssalden in meiner Simulation untersuche, dann unterschätze ich tendenziell die positiven Auswirkungen erhöhter Einwanderung auf die Altersstruktur. Meine Variante mit einem Migrationssaldo von 400000 jährlichen Einwanderern kann demnach als untere Schranke für die positiven Effekte angesehen werden.

Abbildung 3.1: Alterszusammensetzung der Einwanderer und der Einheimischen im Jahr 1989

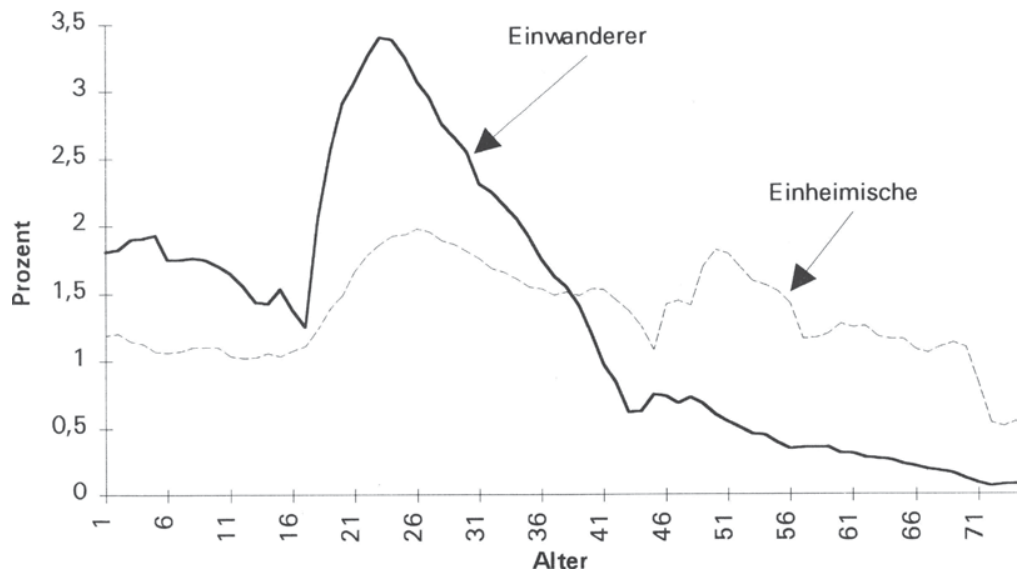

Quelle: Statistisches Bundesamt

2 Die Tabelle "Wanderungen über die Grenzen des Bundesgebietes 1989 nach Altersjahren" wurde mir vom Statistischen Bundesamt freundlicherweise zur Verfügung gestellt. 
Abbildung 3.1 zeigt die Alterszusammensetzung der Einwanderer im Vergleich zur Alterszusammensetzung der Einheimischen Bevölkerung und macht deutlich, $\mathrm{da} ß$ erhöhte Migrationssalden die Bevölkerungsstruktur erheblich verjüngen können.

Die Bevölkerungsprognose beruht auf dem folgenden Programm: Aus den Fertilitätsraten werden die Neugeboren berechnet, die geschlechts- und altersspezifischen Mortalitätsraten verringern die Bevölkerung des Vorjahres und der Migrationssaldo wird geschlechts- und altersspezifisch der einheimischen Bevölkerung zugeteilt. Die Basisvariante meiner Bevölkerungsprognose geht aus von:

- konstanten Fertilitätsraten, die einer Nettoreproduktionsrate von 1,39 entsprechen,

- weiter steigender durchschnittlicher Lebenserwartung Neugeborener, die im Jahr 2050 80,3 Jahre erreicht und

- einem konstanten Migrationssaldo von 200000 Einwanderern nach dem Jahr 2000.

Abbildung 3.2: Bevölkerungsstruktur in Westdeutschland in den Jahren 1989 und 2040

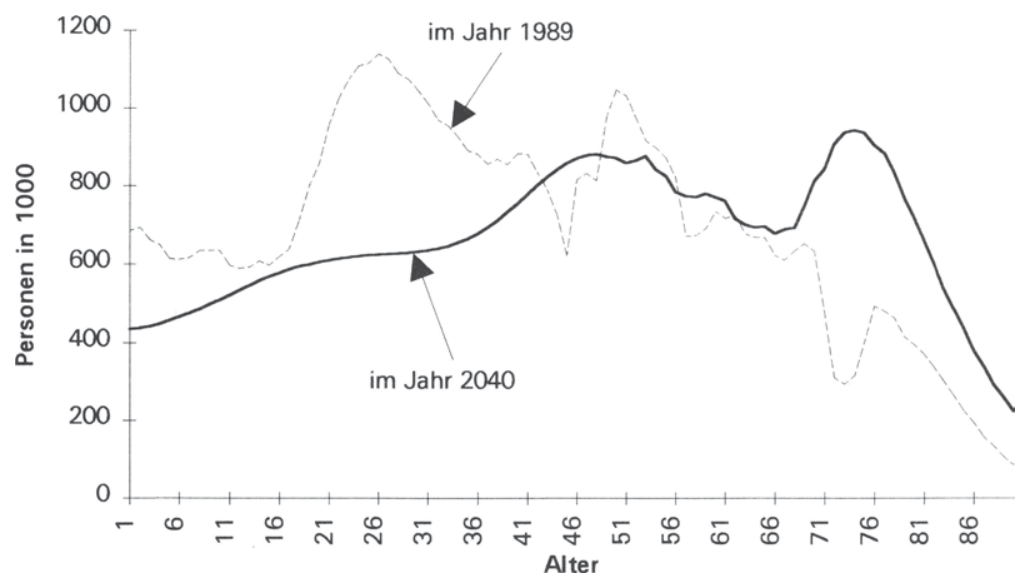

Quelle: Statistisches Bundesamt (1991b) und eigene Bevölkerungsprognose (Basisvariante) 
Abbildung 3.2 zeigt die Anzahl der Personen eines Alters im Jahr 2040 bei der Migrationsvariante mit 200.000 Einwanderern. Zum Vergleich ist die Bevölkerungsstruktur im Basisjahr 1989 ebenfalls abgebildet.

Die Bevölkerungsstruktur im Jahr 2040 zeichnet sich durch einen deutlich höheren Anteil älterer Menschen und einen dementsprechend geringeren Anteil der jüngeren Generation aus. Im Detail zeigt die Bevölkerungsstruktur von 1989 die geburtenschwachen Jahrgänge während der beiden Weltkriege sowie die geburtenstarken Jahrgänge von ungefähr 1935 bis 1940 und 1958 bis 1967, letztere sind die Babyboom-Generation. Im Jahr 2040 hat die Babyboom-Generation ein Alter zwischen siebzig und achtzig Jahren erreicht und ist in Abbildung 3.2 deutlich zu erkennen. Den zweiten Buckel in der Bevölkerungsstruktur von 2040 bilden die Kinder der Babyboom-Generation, die allerdings über einen größeren Altersbereich verteilt sind, weil sich die Geburten der Frauen nicht auf ein spezielles Alter konzentrieren, sondern auf zwanzig Jahre - zwischen achtzehn und achtunddreißig - verteilen. Aus dem gleichen Grund ist ein dritter Buckel für die Enkel der Babyboom-Generation in der Bevölkerungsstruktur von 2040 kaum noch festzustellen.

\section{Abbildung 3.3: Anteil der Kinder, Erwerbspersonen und Personen im Rentenalter an der Gesamtbevölkerung in Westdeutschland zwischen 1996 und 2050}

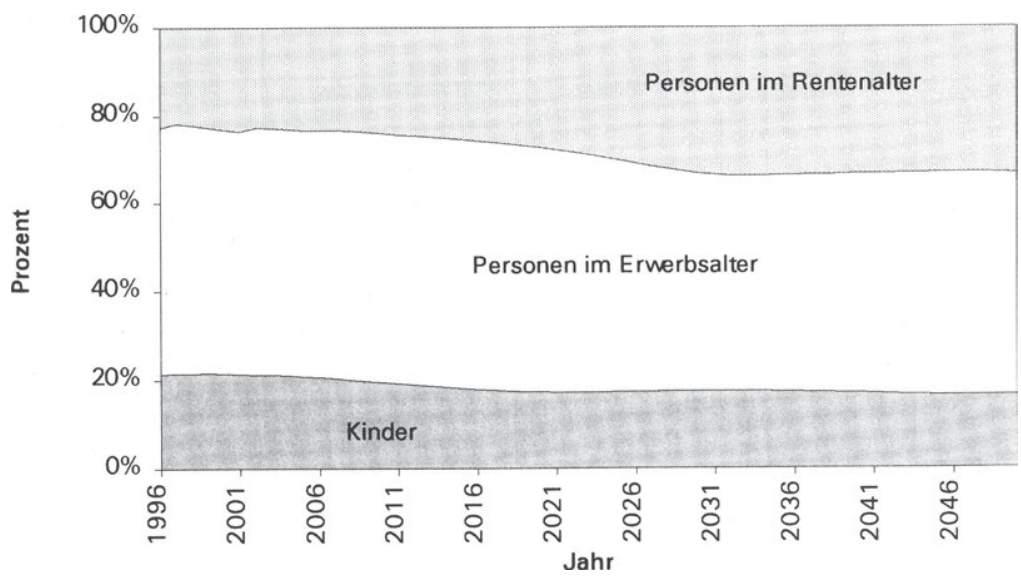

Quelle: Eigene Bevölkerungsprognose (Basisvariante) 
Abbildung 3.2 gibt die Bevölkerungsstruktur in zwei einzelnen Jahren wieder. Einen groben Eindruck von der Bevölkerungsentwicklung zwischen 1996 und 2050 vermittelt Abbildung 3.3. Sie zeigt, wie sich die Anteile der Kinder bis 20 Jahre, der Personen im Erwerbsalter und der Personen im Rentenalter verändern.

Der Anteil der Kinder sinkt zwischen dem Maximum von 21,5 \% im Jahr 1999 und dem Minimum von 16,4\% im Jahr 2049 kontinuierlich. Deutlicher schlagen sich die demographischen Veränderungen in den Anteilen der Erwerbspersonen und Rentner nieder. Die Erwerbspersonen haben 1997 mit 56,9\% den höchsten Anteil an der Gesamtbevölkerung und gleichzeitig haben die Rentner mit 21,7 \% den niedrigsten Anteil. Ebenfalls gleichzeitig, im Jahr 2033, erreichen beide Kurven die jeweils anderen Extremwerte: 48,9\% ist der minimale Anteil der Erwerbstätigen und 33,7\% ist der maximale Anteil der Rentner. Diese Entwicklung wird durch die Babyboom-Generation getragen, die bis zum Jahr 2029 aus dem Erwerbsleben ausgeschieden ist.

Die Gesamtbevölkerung verändert sich bis zum Jahr 2017 nur wenig, sie bleibt bei 66 bis 67 Millionen Einwohnern in Westdeutschland. Danach fällt sie kontinuierlich und erreicht im Jahr 2050 mit 57,6 Millionen Einwohnern den niedrigsten Wert in dem betrachteten Zeitraum.

\subsubsection{Bevölkerung und Haushalte}

Raffelhüschen und Kitterer (1990) fassen die einzelnen Haushalte zu sieben Generationen zusammen. Wesentlich tiefer unterteilen Auerbach et al. (1989) den Haushaltssektor, in dem sie jede Kohorte (d.h. jedes Lebensjahr von 21 bis 75) durch einen repräsentativen Haushalt beschreiben. Weil der Alterungsproze $B$ die Kohortenverteilung ganz entscheidend verändert und seine makroökonomischen Auswirkungen das Thema meiner Arbeit sind, orientiere ich mich an Auerbach et al. und untergliedere den Haushaltssektor in Kohorten. Jede Kohorte wird durch einen repräsentativen Haushalt vertreten, der mit der Anzahl der Haushalte dieser Kohorte gewichtet wird. Also muß die Bevölkerungsprognose um eine Verbindung zwischen Personen und Haushalten erweitert werden. Dazu nehme ich an, $\mathrm{da} ß$ alle Personen einen einheitlichen Lebenszyklus haben, unabhängig von ihrem Geburtsjahr: Bis zur Vollendung des 20. Lebensjahres leben die Kinder im Haushalt ihrer Eltern. Mit Beginn des 21. Lebensjahres heiraten die nun erwachsenen Kinder einen Partner gleichen Alters ${ }^{3}$, gründen einen eigenen Haushalt und beginnen ihr Erwerbsleben. Am Ende des 27. Lebensjahres unmittelbar vor dem Be-

3 Weil der Haushalt aus zwei Mitgliedern gleichen Alters besteht, definiere ich das „Haushaltsalter“ als das Lebensjahr der Mitglieder. 
ginn des 28. Lebensjahres, dem durchschnittlichen Entbindungsalter von Müttern in Westdeutschland ${ }^{4}$, bekommt das Paar gleichzeitig alle Kinder, sozusagen als Zwillinge. Folglich beträgt die Generationszeit in diesem einheitlichen Lebenszyklus 27 Jahre. Vom 28. bis zum 47. Lebensjahr besteht der Haushalt aus den beiden Eltern und ihren Kindern. Mit dem 48. Lebensjahr gründen die Kinder ihren eigenen Haushalt, so daß die Eltern bis zu ihrem Tod am Ende des Lebensjahres $T$ - 1 wieder zu zweit einen Haushalt führen.

Der für alle Personen einheitliche Lebenszyklus ist in Tabelle 3.2 schematisch dargestellt, dabei bezeichnet $k$ das Geburtsjahr einer Person (also die Kohorte), $t$ das Lebensjahr und $n(k, t)$ die Anzahl der Personen im Alter von $t$, die zur Kohorte $k$ gehören. Die Matrix $n$ ist das Ergebnis der Bevölkerungsprognose und enthält die Kohortenstärke für jedes Lebensjahr.

Tabelle 3.2: Zusammensetzung, durchschnittliche Größe und Anzahl der Haushalte einer Kohorte $k$

\begin{tabular}{|c|c|c|c|}
\hline $\begin{array}{c}\text { Lebensjahr } \\
t\end{array}$ & $\begin{array}{c}\text { Zusammensetzung } \\
\text { des Haushalts }\end{array}$ & $\begin{array}{c}\text { durchschnittliche } \\
\text { Haushaltsgröße } h\end{array}$ & $\begin{array}{c}\text { Anzahl der } \\
\text { Haushalte }\end{array}$ \\
\hline \hline $1-20$ & bei den Eltern & bei den Eltern & 0 \\
\hline $21-27$ & Paar & 2 & $n(k, t) / 2$ \\
\hline $28-47$ & Paar mit Kindern & $2 \cdot(1+n(k+27, t-27) / n(k, t))$ & $n(k, t) / 2$ \\
\hline $48-(T-1)$ & Paar & 2 & $n(k, t) / 2$ \\
\hline
\end{tabular}

Quelle: Eigene Darstellung

Tabelle 3.2 stellt die Verbindung zwischen der Bevölkerungsprognose und dem Haushaltssektor her, denn die durchschnittliche Haushaltsgröße $h$ jeder Kohorte geht in die Lebensnutzenfunktion (3.3) der Haushalte ein. In den meisten Lebensjahren besteht der Haushalt aus zwei Personen - unabhängig von der zu repräsentierenden Kohorte. Kohortenspezifisch und abhängig von den Geburtenzahlen ist die durchschnittliche Haushaltsgröße in den Jahren, in denen die Kinder bei den Eltern wohnen, also zwischen dem 28. und 47. Lebensjahr der Eltern. Weil nur die Eltern im erwerbsfähigen Alter sind, gilt in allen Lebensjahren für die Anzahl

4 Das durchschnittliche Entbindungsalter von Müttern in Westdeutschland (das sind ausländische und deutsche Mütter) ergibt sich aus der „Tabelle der im Jahr 1989 Lebendgeborenen nach dem Alter der Mutter", die im Statistischen Jahrbuch 1991 veröffentlicht ist, siehe Statistisches Bundesamt (1991b). 
der Erwerbspersonen eines Haushalts $b=2$. Die in der letzten Spalte von Tabelle 3.2 ausgewiesene Anzahl der Haushalte einer Kohorte ist wichtig für die Aggregation der Kohorten, aus der das gesamtwirtschaftliche Kapital- und Arbeitsangebot sowie die gesamtwirtschaftliche Konsumnachfrage des Haushaltssektors entstehen.

Die in Tabelle 3.2 unterstellte Verbindung zwischen Bevölkerungsprognose und Haushalten schafft die Struktur, mit der ich die Bevölkerungsprognose in die Simulation des allgemeinen Gleichgewichts einbinde. Allerdings blendet diese Stilisierung einige Aspekte des demographischen Wandels aus, die sich in der Haushaltszusammensetzung niederschlagen. Durch den angenommen einheitlichen Lebenszyklus beträgt die Generationszeit aller Haushalte und aller Kohorten 27 Jahre. In der Realität ist jedoch eine Zunahme der Generationszeit zu beobachten, d.h. das durchschnittliche Entbindungsalter von Müttern steigt. Ausgelöst wird dieser Trend unter anderem dadurch, daß Frauen und Männer mehr Zeit für ihre Ausbildung aufbringen. So haben zum Beispiel die zwischen 1932 und 1937 geborenen Jahrgänge zu $80 \%$ höchstens den Hauptschulabschluß, zu $12 \%$ den Realschulabschluß und zu $8 \%$ das Abitur. Für die zwischen 1962 und 1967 geborenen Kohorten haben sich die Verhältnisse völlig geändert: Nur noch $45 \%$ haben höchstens den Hauptschulabschluß, $30 \%$ den Realschulabschluß und $25 \%$ das Abitur (siehe Dustmann und Meier, 1995). Wenn man davon ausgeht, daß dieser Trend wahrscheinlich abgeschwächt anhalten wird, dann wird die Generationszeit auch in Zukunft steigen. Von diesem Anstieg abstrahiert Tabelle 3.2. Ein weiterer Aspekt des demographischen Wandels betrifft die Haushaltsgröße, die in den letzten Jahren deutlich abgenommen hat. Zwischen 1970 und 1990 ist der Anteil von Ein-Personen-Haushalten von $25 \%$ auf $35 \%$ gestiegen und der Anteil von Haushalten mit fünf und mehr Personen von fast $13 \%$ auf etwas mehr als $5 \%$ gesunken (siehe Statistisches Bundesamt, 1975b und 1992b). Ansatzweise trägt die in Tabelle 3.2 unterstellte Haushaltsgröße dieser Beobachtung Rechnung, indem sie wegen der niedrigeren Kinderzahl die durchschnittliche Haushaltsgröße im Alter von 28 bis 47 Jahren verringert. Es ist aber unbestritten, daß Tabelle 3.2 den in der Realität beobachteten Wandel der Haushaltsstrukturen unvollkommen wiedergibt.

Vergleicht man die Haushaltsstruktur in Tabelle 3.2 mit den Haushaltsstrukturen anderer Simulationen, so stellt man fest, daß die dort getroffenen Annahmen zum Teil sehr viel restriktiver sind. Cutler et al. (1990) sowie Börsch-Supan (1995a, 1996) stellen die Verbindung zwischen der Bevölkerungsentwicklung und der Makroökonomie durch den Altersquotienten her, eine explizite Verbindung zwischen Personen und Haushalten gibt es in ihren Arbeiten nicht. Raffelhüschen und Kitterer (1990) gehen etwas weiter, indem sie die Bevölkerung in sieben Generationen zusammenfassen. Buslei und Kraus (1996) modellieren keine Haushalte, sie betrachten Singles. Eine sehr viel feinere Untergliederung liefern Auerbach et 
al. (1989), deren Haushaltsstruktur der in Tabelle 3.2 angenommenen Haushaltsstruktur sehr nahe kommt. Allerdings gehen sie von einer sicheren Lebenserwartung aus - eine zweifellos restriktive Annahme, die ich in meiner Simulation aufhebe.

\subsubsection{Tod: Vererben und Erben}

Die Bevölkerungsprognose verringert die Anzahl der Personen eines Alters mit den entsprechenden Mortalitätsraten. Im allgemeinen Gleichgewicht muß genau definiert werden, was mit dem Vermögen der verstorbenen Personen geschieht. Dazu unterscheide ich zwei Fälle: Im ersten Fall sterben die Eltern, bevor die Kinder den elterlichen Haushalt verlassen und im zweiten Fall leben die Kinder beim Tod ihrer Eltern schon in einem eigenen Haushalt. Im zweiten Fall nehme ich an, daß die Kinder das Vermögen ihrer Eltern in gleichen Teilen erben. Der erste Fall ist etwas schwieriger, denn die Kinder leben noch nicht in einem eigenen Haushalt. Deshalb nehme ich an, daß die Geschwister der Eltern (die wegen des unterstellten Lebenszyklusses der gleichen Kohorte wie die verstorbenen Eltern angehören) die Kinder aufnehmen und das Vermögen der Eltern bekommen. Tabelle 3.3 baut auf dem in Tabelle 3.2 dargestellten einheitlichen Lebenszyklus auf und faßt die Erbschaftsbeziehungen zusammen. Die Matrix a enthält das durchschnittliche Vermögen der Kohorte $k$ im Lebensjahr $t$.

Tabelle 3.3: Erbschaftsbeziehungen für eine Kohorte $k$

\begin{tabular}{|c|c|c|c|}
\hline Lebensjahr $t$ & $\begin{array}{c}\text { Vererben } \\
\text { an: }\end{array}$ & $\begin{array}{c}\text { Erbschaft } \\
\text { erhalten von: }\end{array}$ & $\begin{array}{c}\text { Durchschnittliche Höhe der } \\
\text { erhaltenen Erbschaft }\end{array}$ \\
\hline $21-47$ & Geschwister & $\begin{array}{c}\text { Eltern und } \\
\text { Geschwistern }\end{array}$ & $\begin{array}{c}\frac{n(k-27, t+26)-n(k-27, t+27)}{m(k, t)} e(k-27, t+27) \\
+\frac{n(k, t-1)-n(k, t)}{n(k, t)} \theta(k, t)\end{array}$ \\
\hline $48-(T-27)$ & Kinder & Eltern & $\frac{n(k-27, t+26)-n(k-27, t+27)}{m(k, t)} a(k-27, t+27)$ \\
\hline$(T-26)-T$ & Kinder & - & 0 \\
\hline
\end{tabular}

Quelle: Eigene Darstellung

Genau wie Tabelle 3.2 den Zusammenhang zwischen Bevölkerungsprognose und Haushaltszusammensetzung stilisiert, vereinfacht auch die in Tabelle 3.3 angenommene Erbschaftsstruktur die Realität. Beispielsweise gibt es in meinem Modell weder Vererbungen von Großeltern an ihre Enkel, noch inter vivos Transfers. Diese beiden Vererbungsformen sind in der Realität nicht zuletzt wegen der Erb- 
schaftssteuer interessant. Damit ergibt sich ein weiterer Punkt, denn die Erbschaftssteuer wird hier nicht thematisiert. In meiner Simulation gibt es keinen Staat der Steuern erhebt, es existiert nur eine Rentenversicherung. Hier ergibt sich eine mögliche Erweiterung meines Modells: Man könnte eine Erbschaftssteuer einführen, um damit einen Teil der Renten zu finanzieren. Wenn das Erbschaftsvolumen steigt, was durch das Ableben der geburtenstarken Jahrgänge zwischen 2030 und 2050 der Fall sein wird, dann könnte der Druck auf die lohnbezogenen Beitragssätze der Rentenversicherung durch eine Erbschaftssteuer gemildert werden. Allerdings würde eine Erbschaftssteuer ähnlich wie die lohnbezogenen Beitragssätze zu Effizienzverlusten führen, indem sie die MRSA verzerrt.

Ein wichtiger Punkt in Tabelle 3.3 sind die Vererbungen an Geschwister. Da ich jede Kohorte durch einen repräsentativen Haushalt abbilde, werden die innerhalb einer Kohorte transferierten Erbschaften endogen und müssen daher bei der Optimierung beachtet werden. Dies ist in meinem Haushaltsmodell korrekt berücksichtigt.

\subsection{Der Produktionssektor}

Das Firmenverhalten bilde ich in einem stark stilisierten Modell ab. Es gibt viele identische Firmen, die untereinander konkurrieren. Aus der Sicht jeder einzelnen Firma sind Zins- und Lohnsatz gegeben. Alle Firmen produzieren mit den homogenen Produktionsfaktoren Kapital $K$ und Arbeit $L$ das homogene Gut $Y$; der Produktionsproze $ß$ wird durch eine Produktionsfunktion $F$ abgebildet, die den InadaBedingungen $^{5}$ genügt. Der Kapitalstock $K$ wird mit der konstanten Rate $\delta$ abgeschrieben und kann ohne Kosten neuen Bedingungen angepaßt werden, d.h. Investitionskosten fallen nicht an. Wegen dieser Annahme reduziert sich die intertemporale Optimierung der Firmen zu einer „statischen“ Optimierung, die jede Periode getrennt betrachtet. Im Gewinnmaximum wählen die Firmen die Produktionsfaktoren so, daß die marginalen Produkte genau den marginalen Kosten entsprechen. Mit $F_{K}$ für das marginale Produkt des Kapitals und $F_{L}$ für das marginale Produkt der Arbeit gilt:

5 Eine Produktionsfunktion $F$ erfüllt die Inada-Bedingungen wenn gilt:

- $F(\cdot)$ hat positives und abnehmendes Grenzprodukt bezüglich jedes Produktionsfaktors.

- $F(\cdot)$ ist linear-homogen: Für alle $\lambda>0$ ist $F(\lambda K, \lambda L)=\lambda \cdot F(K, L)$

- Das Grenzprodukt jedes Produktionsfaktors geht gegen null, wenn der entsprechende Produktionsfaktor gegen unendlich geht und geht gegen unendlich, wenn der entsprechende Produktionsfaktor gegen null geht. 


$$
r+\delta=F_{K}
$$

$$
w=F_{L}=\frac{Y}{L}-(r+\delta) \frac{K}{L}
$$

Weil ich annehme, daß alle Firmen gleich sind, können sie zu einer repräsentativen Firma aggregiert werden. Diese repräsentative Firma steht für den ganzen Produktionssektor und bestimmt mit Gleichungen (3.11) und (3.12) bei gegebenen Zins- und Lohnsätzen die gesamtwirtschaftliche Arbeits- und Kapitalnachfrage.

Für das Simulationsmodell wähle ich eine Cobb-Douglas Produktionsfunktion:

$$
Y=K^{\alpha} L^{1-\alpha} \quad \text { mit } \alpha \in(0,1)
$$

Die Produktionsfunktion (3.13) abstrahiert von arbeitssparendem technischem Fortschritt. Arbeitssparender technischer Fortschritt ist die einzige Form des technischen Fortschritts, die mit konstanten Wachstumsraten im Steady-State vereinbar ist (siehe den Beweis in Barro und Sala-i-Martin (1995), Anhang zum ersten Kapitel). Bei endogenem Arbeitsangebot führt arbeitssparender technischer Fortschritt langfristig zu problematischen Ergebnissen. Das Problem wird an der MRSL (Gleichung 3.7) deutlich: Wenn die Arbeit $L$ in der Produktionsfunktion (3.13) durch effiziente Arbeitseinheiten $e L$ ersetzt wird und die Arbeitseffizienz $e$ durch den technischen Fortschritt jährlich wächst, dann steigt das Grenzprodukt der Arbeit, was nach Gleichung (3.12) steigenden Lohn bedeutet. Also steigt bei technischem Fortschritt das nutzenerhaltende Tauschverhältnis von Freizeit und Konsum - die MRSL - stetig an, so daß der Verzicht auf Freizeit mit immer mehr Konsumeinheiten ausgeglichen werden muß. Nun ist der Substitutionseffekt aber eine Bewegung auf der Indifferenzkurve. Wenn die Indifferenzkurve konkav zum Ursprung ist, dann wird bei steigendem Lohn Freizeit gegen Konsum substituiert. Weil der Betrag der MRSL dem Lohn gleicht, wird bei steigendem Lohn aber immer weniger Freizeit für immer mehr Konsum eingeschränkt - bildlich gesprochen befindet man sich in einem zunehmend steilen Bereich der Nutzenfunktion. Wegen der in diesem Bereich nur noch sehr geringen Einschränkung der Freizeit wird der Einkommenseffekt das Geschehen bestimmen: Der geringen Substitutionsreaktion, welche die Freizeit reduziert, steht ein großer Einkommenseffekt gegenüber, der die Freizeit erhöht. Folglich würde das Arbeitsangebot langfristig stark zurückgehen.

Auerbach et al. (1989) umgehen das Problem „technischer Fortschritt“ mit einem Trick: Sie erhöhen von Jahr zu Jahr die maximal verfügbare Zeit. Die Argumentation von Auerbach et al. überzeugt mich allerdings nicht, denn modelltheoreti- 
sch sind technischer Fortschritt und maximal verfügbare Zeit durchaus unterschiedlich zu sehen: Technischer Fortschritt greift in den Produktionsprozeß ein, aber das Zeitbudget ist eine Restriktion des Haushaltssektors. Raffelhüschen (1993) wählt einen anderen Weg: Er faßt die Wachstumsrate der Bevölkerung (konstante $-1,5 \%$ ) und die Rate des arbeitssparenden technischen Fortschritts $(2,5 \%)$ zu einem Parameter zusammen. Auch diesen Vorschlag halte ich nicht nur wegen der konstanten Bevölkerungsabnahme für wenig überzeugend.

Das Problem technischer Fortschritt bei endogenem Arbeitsangebot könnte ein lohnendes Gebiet für zukünftige Arbeiten sein. Da der Schwerpunkt meiner Simulationen ein Vergleich verschiedener Politikszenarien ist, halte ich es für vertretbar, aus den erwähnten Gründen von technischem Fortschritt abzusehen. Der technische Fortschritt würde sich in den Szenarien relativ ähnlich niederschlagen, so daß die vergleichende Analyse der Szenarien durch den technischen Fortschritt kaum verändert würde. Ich kann allerdings keine Aussagen darüber machen, ob und wie die durch den technischen Fortschritt entstehende Wohlfahrtssteigerung zur Milderung der Rentenproblematik dienen könnte.

Weil die Cobb-Douglas Produktionsfunktion (3.13) eine linear-homogene Funktion ist, können Zinssatz, Lohn und Output pro Arbeitnehmer sehr einfach mit der Kapitalintensität ${ }^{6} x=K / L$ beschrieben werden, denn es gilt:

$$
y=\frac{Y}{L}=\left(\frac{K}{L}\right)^{\alpha}=x^{\alpha}
$$

$r+\delta=\alpha x^{\alpha-1}$

$$
w=y-(r+\delta) x=(1-\alpha) x^{\alpha}=(1-\alpha) y
$$

Gleichung (3.15) begründet eine negative Korrelation zwischen der Kapitalintensität $x$ und dem Zinssatz $r$, denn die Kapitalelastizität $\alpha$ liegt zwischen null und eins. Dagegen ist der Zusammenhang zwischen der Kapitalintensität $x$ und dem Output $y$ sowie dem Lohn $w$ positiv: Steigendes Kapital pro Arbeitnehmer erhöht den Output pro Arbeitnehmer und den Lohn.

6 Weil das Symbol $k$ für das Geburtsjahr einer Kohorte schon vergeben ist, bezeichne ich die Kapitalintensität mit $x$, um Verwechslungen zu vermeiden. 


\subsection{Die Rentenversicherung}

In meinem Simulationsmodell hat der Staat eine einzige Funktion: Er ist Träger einer umlagefinanzierten Rentenversicherung. Im Umlageverfahren müssen in jedem Jahr die Einnahmen gleich den Ausgaben sein, das Budget der Rentenversicherung kann also weder Defizite noch Überschüsse aufweisen. Die Rentenversicherung wird durch Beiträge finanziert, die proportional vom Bruttolohn abgezogen werden und für alle Beschäftigten gleich sind, also einer Lohnsteuer mit einheitlichem Steuersatz entsprechen. Bei gegebenen Ausgaben (Rentenzahlungen) muß der Staat in jedem Jahr den Beitragssatz neu bestimmen, damit die Budgetrestriktion der Rentenversicherung erfüllt ist.

Die Rentenzahlungen werden im deutschen Rentensystem durch die Nettolohnentwicklung und die Rentenformel bestimmt. In die Rentenformel gehen die relative Einkommensposition des Arbeitnehmers in jedem Beitragsjahr, die Anzahl der Beitragsjahre und das Verrentungsalter ein. Weil das Simulationsmodell auf Kohorten aufbaut, fällt die individuelle Komponente in der Rentenformel weg, aber der Bezug zur Nettolohnentwicklung und zum Lebensarbeitseinkommen bleibt bestehen. Im Simulationsmodell beträgt das Verrentungsalter TR Jahre, d.h. nach Vollendung des Lebensjahres $T^{R}$ scheiden die Arbeitnehmer aus dem Erwerbsleben aus. Das Verrentungsalter ist kohortenspezifisch und exogen vorgegeben, kann aber von der Politik geändert werden. Eine spätere Verrentung schließe ich aus. Schaut man sich Verrentungsprofile für Westdeutschland an, so erkennt man deutliche Spitzen im Alter von 60, 63 und 65 Jahren, die vor allem durch die institutionellen Regelungen des Rentenversicherungssystems bestimmt werden (siehe Schmidt, 1995). Insofern halte ich die exogene Vorgabe des Verrentungsalters für vertretbar. Vorruhestandsregelungen berücksichtige ich nicht explizit. Bei endogenem Arbeitsangebot können die Arbeitnehmer jederzeit freiwillig ihr Arbeitsangebot zurücknehmen.

Andere Arbeiten, beispielsweise Raffelhüschen und Kitterer (1990) sowie Buslei und Kraus (1996), verzichten auf ein exogen gegebenes Verrentungsalter und erscheinen zunächst realistischer. Tatsächlich ist diese Verallgemeinerung aber wenig überzeugend, denn Raffelhüschen und Kitterer erhöhen beim Übergang in die Ruhestandsphase die vorher konstante Freizeitintensität und erreichen so ein drastischen Rückgang des Arbeitsangebots. Buslei und Kraus unterstellen einen Lohnpfad, der ab dem 60. Lebensjahr auf geringem Niveau konstant bleibt. Dieser Lohnpfad ist exogen gegeben und verursacht einen ähnlichen Effekt wie die steigende Freizeitintensität bei Raffelhüschen und Kitterer: Nach dem 60. Lebensjahr geht das Arbeitsangebot sehr stark zurück. Ob diese Art der Modellierung der Realität näher kommt als eine durch institutionelle Regelungen nicht ganz unplausibel erscheinende exogene Festsetzung des Verrentungsalters, halte ich für fraglich. 
In meinem Modell der umlagefinanzierten Rentenversicherung beziehen die ehemaligen Arbeitnehmer ab dem Lebensjahr $T P=T R+1$ eine Rente, die sich aus dem persönlichen Rentenwert und der Entwicklung des Nettolohns ergibt. Der persönliche Rentenwert einer Kohorte $k$ berechnet sich aus der vom Staat für das erste Jahr des Ruhestands, $k+T^{P}$, festgelegten Netto-Ersatzquote $q_{k+T p}$ und dem Netto-Lebensarbeitseinkommen wlife $_{k}$. Formel (3.17) gibt die Rente an, die ein Arbeitnehmer im ersten Jahr seines Ruhestands erhält:

$$
w_{k, T^{P}}^{R}=q_{k+T^{p}} \cdot w_{\text {life }}
$$

$\mathrm{Zu}$ beachten ist die Indexierung: Das Lebensarbeitseinkommen ist kohortenspezifisch und steht zum betrachteten Zeitpunkt bereits fest, so daß wlife durch $k$ eindeutig indexiert ist. Die Netto-Ersatzquote gilt für ein bestimmtes Jahr, das sich aus dem Geburtsjahr $k$ und dem Alter der Kohorte im ersten Jahr des Ruhestands $T^{P}$ ergibt; die Rentenhöhe $W^{R}$ hängt folglich von der Kohorte und dem Alter ${ }^{7} \mathrm{ab}$. Die Netto-Ersatzquote kann sich von Jahr zu Jahr ändern, wodurch die neu in den Ruhestand eintretenden Kohorten betroffen werden. Alle Kohorten die sich schon im Ruhestand befinden behalten dagegen ihr Rentenniveau, das in den Jahren $j$, die dem Jahr $T^{P}$ folgen, entsprechend der Nettolohnentwicklung ( $w$ bezeichnet ab jetzt den Nettolohn) angepaßt wird:

$$
w_{k, t}^{R}=\frac{w_{j}}{w_{j-1}} \cdot w_{k, t-1}^{R}=\frac{w_{j}}{w_{k+T^{P}}} \cdot q_{k+T^{P}} \cdot w_{\text {life }} k_{k}
$$

Weil die Einnahmen und Ausgaben der Rentenversicherung in jedem Jahr $j$ gleich sein sollen, muß bei gegebenen Ersatzquoten $q_{i}$ für $i \leq j$ der Beitragssatz $\sigma_{j}$ entsprechend gewählt werden. Die Einzahlungen $E Z$ eines Jahres $j$ ergeben sich aus den Beitragszahlungen der Erwerbstätigen. Die Anzahl der Erwerbstätigen einer Kohorte errechnet sich aus der Anzahl der Haushalte. Nach Tabelle 3.1 ist die Anzahl der Haushalte in Kohorte $k$, deren Erwachsene in Jahr $j$ ein Alter von $t$ haben, genau die Hälfte der Bevölkerung $n(k, t)$ mit entsprechendem Geburtsjahr und Alter. Jede Kohorte $k$ im Erwerbsalter wird durch einen Haushalt repräsentiert, der im Jahr $j$ gemäß seinen Präferenzen $\left(2-I_{k, j-k}\right)$ Arbeitseinheiten anbietet. Die Summe der Einzahlungen ist dann:

$$
E Z_{j}=\sum_{k=j-21}^{j-T^{R}} \sigma_{j} \cdot w_{j}^{B} \cdot\left(2-I_{k, j-k}\right) \cdot \frac{n(k, j-k)}{2}=\sigma_{j} \cdot w_{j}^{B} \cdot L_{j}
$$

7 Generell gilt zwischen der Kohorte $k$, dem Alter $t$ und dem aktuellen Jahr $j$ der Zusammenhang $j=k+t$. 
Hierbei bezeichnet $w^{B}$ den Bruttolohn und $L$ das aggregierte Arbeitsangebot. Die Ausgaben $A Z$ der Rentenversicherung in Jahr $j$ sind die Summe der Zahlungen an die Rentner. Da die Rentenhöhe einerseits von der im Jahr der Verrentung geltenden Ersatzquote (die $w^{R}$ bestimmt) und andererseits von der Nettolohnentwicklung abhängt, summieren sich die Renten zu:

$$
\begin{aligned}
A Z_{J} & =\sum_{k=j-T^{p}}^{l-T+1} \frac{\left(1-\sigma_{j}\right) w_{j}^{B}}{\left(1-\sigma_{k+T^{P}}\right) w^{B}{ }_{k+T^{P}}} w^{R}{ }_{k+T^{p}} \frac{n(k, j-k)}{2} \\
& =w^{R}{ }_{j} \frac{n\left(k, T^{P}\right)}{2}+\left(1-\sigma_{j}\right) w_{j}^{B} \cdot \sum_{k=j-T^{P}-1}^{j-T+1} \frac{w^{R}{ }_{k+T^{P}}}{\left(1-\sigma_{k+T^{P}}\right) w^{B}{ }_{k+T^{P}}} \frac{n(k, j-k)}{2}
\end{aligned}
$$

Gleichung (3.20) läßt sich sehr einfach nach dem gesuchten Beitragssatz $\sigma_{j}$ auflösen, denn die Summe auf der rechten Seite enthält nur die Beitragssätze vergangener Jahre, die bei der Bestimmung von $\sigma_{j}$ schon bekannt sind. Zur Vereinfachung der Schreibweise definiere ich die Hilfsgrößen SP1 und SP2:

$$
\begin{aligned}
& S P 1_{j}=w_{j}^{R} \frac{n\left(k, T^{P}\right)}{2} \\
& S P 2_{j}=\sum_{k=j-T^{P}-1}^{j-T+1} \frac{w_{k+T^{P}}}{\left(1-\sigma_{k+T^{P}}\right) w^{B}{ }_{k+T^{P}}} \cdot \frac{n(k, j-k)}{2}
\end{aligned}
$$

$S P 1_{j}$ sind die Rentenzahlungen an die im Jahr $j$ neu in den Ruhestand eintretenden Haushalte und $S P 2_{j}$ ist die Summe der Rentenzahlungen an die schon länger im Ruhestand weilenden Kohorten. Die Budgetrestriktion der Rentenversicherung - die Bedingung $A Z_{j}=E Z_{j}$ - kann jetzt problemlos nach $\sigma_{j}$ aufgelöst werden:

$$
\sigma_{j}=\frac{S P 1_{j} / w_{j}{ }^{B}+S P 2_{j}}{L_{j}+S P 2_{j}}
$$

In das gerade erarbeitete Rentenversicherungssystem kann auch eine kapitalgedeckte Rentenversicherung integriert werden. Wenn die Netto-Ersatzquote $q$ und der Beitragssatz $\sigma$ null sind, müssen die Haushalte ihren Konsum im Alter aus Ersparnissen finanzieren, die sie während ihres Erwerbslebens beispielsweise in einer staatlichen kapitalgedeckten Rentenversicherung oder privatwirtschaftlichen Lebensversicherung gebildet haben. Das im folgenden Abschnitt beschriebene allgemeine Gleichgewichtsmodell unterscheidet nicht zwischen verschiedenen Assets, d.h. es unterstellt, daß alle finanziellen Aktiva die gleiche Verzinsung erhalten. Weiterhin gibt es in der idealisierten Welt des allgemeinen Gleichgewichts 
keine Transaktions- oder Verwaltungskosten, so daß die Frage, ob eine kapitalgedeckte Rentenversicherung staatlich oder privatwirtschaftlich organisiert wird, hier nicht von Bedeutung ist.

Ein weiterer Aspekt der in der Realität existierenden Rentenversicherung wird durch die Aggregation ausgeblendet: Da jede Kohorte durch einen repräsentativen Haushalt abgebildet wird, gibt es in der gerade modellierten Rentenversicherung keine Umverteilung innerhalb der Generationen. Durch Vorruhestandsregelungen, Ausbildungs- und Erziehungszeiten, aber auch Hinterbliebenenregelungen finden solche intragenerationalen Umverteilungen in der deutschen Rentenversicherung statt. Dagegen wird die für meine Arbeit entscheidende Frage der intergenerationalen Umverteilung durch den Generationenvertrag, der sich in der Budgetrestriktion der umlagefinanzierten Rentenversicherung niederschlägt, berücksichtigt.

\subsection{Allgemeines Gleichgewicht}

Das Simulationsmodell bestimmt die Zinssätze, die Löhne und die Beitragssätze in jeder Periode so, daß auf allen Märkten Angebot und Nachfrage übereinstimmen. Zusätzlich haben die beteiligten Wirtschaftssubjekte perfekte Voraussicht, also müssen im allgemeinen Gleichgewicht die ex ante Erwartungen der Wirtschaftssubjekte mit den realisierten Größen ex post übereinstimmen.

Drei Märkte bestimmen die Ökonomie: der Arbeitsmarkt, der Kapitalmarkt und der Markt für Konsumgüter. Es gibt keine Inflation und das Preisniveau ist auf eins normiert. Wegen des Gesetzes von Walras impliziert ein Gleichgewicht auf zwei Märkten ein Gleichgewicht auf dem dritten Markt. Daher betrachtet das Simulationsmodell nur den Arbeits- und Kapitalmarkt explizit. Die Rentenversicherung und die Bevölkerungsprognose vervollständigen die Ökonomie.

Hinsichtlich der außenwirtschaftlichen Beziehungen untersuche ich die zwei polaren Fälle "geschlossene Volkswirtschaft" und "kleine offene Volkswirtschaft". In der geschlossenen Volkswirtschaft müssen die einheimischen Märkte im Gleichgewicht sein, es gibt keine Einflüsse aus dem Ausland. Dagegen bestimmt in einer kleinen offenen Volkswirtschaft der Weltmarkt-Zinssatz die einheimische Kapitalintensität, die somit aus Sicht der kleinen offenen Volkswirtschaft exogen ist. Mittlere und große offene Volkswirtschaften, zu denen auch Deutschland zu zählen ist, befinden sich zwischen den beiden Randfällen "geschlossene" und „kleine offene Volkswirtschaft“. In der Simulation des allgemeinen Gleichgewichts betrachte ich in verschiedenen Szenarien beide Randfälle, denn so läßt sich die Bandbreite der möglichen Entwicklungen einschätzen. Die Arbeiten von 
Cutler et al. (1990) für zwei Länder sowie Börsch-Supan (1995c, 1995d und 1996) für viele Länder versuchen, die wechselseitigen Abhängigkeiten in einem integrierten Weltmarkt zu modellieren. Allerdings beschreiben diese Arbeiten den Haushaltssektor auf einem sehr hohen Abstraktionsniveau. Diese Vereinfachung erlaubt die Bestimmung eines simultanen Gleichgewichts in den betrachteten Ländern. Weil dies eine erheblich andere Struktur des Simulationsmodells erfordert, beschränke ich mich hier auf die beiden Randfälle - für zukünftige Arbeiten bietet sich hier zweifellos Raum für interessante Erweiterungen. Cutler et al. sowie Börsch-Supan zeigen, daß der demographische Wandel bei integrierten Kapitalmärkten durchaus Chancen eröffnen kann: Wenn Länder mit hohem Bevölkerungswachstum, wie zum Beispiel die südostasiatischen Schwellenländer, aufgrund des Bevölkerungswachstums einen hohen Kapitalbedarf haben, dann ergeben sich für die „alternden“ westlichen Industrieländer Investitionsmöglichkeiten in den Wachstumswirtschaften. Also kann das nicht mehr benötigte Kapital profitabel angelegt werden. Diese Kapitalströme sind sowohl für die westlichen Industrieländer als auch für die Schwellenländer vorteilhaft; eine Erkenntnis, die besonders von Börsch-Supan betont wird.

In meinem Simulationsmodell agieren die Haushalte als Anbieter und die Unternehmer als Nachfrager auf dem Arbeits- und Kapitalmarkt. Der Zinssatz bestimmt die Kapitalnachfrage und der Bruttolohn die Arbeitsnachfrage der Unternehmen. Das Kapitalangebot der Haushalte richtet sich ebenfalls nach dem Zinssatz, aber das Arbeitsangebot orientiert sich am Nettolohn. Die Differenz zwischen Brutto- und Nettolohn entsteht durch den Beitragssatz zur Rentenversicherung, der in Deutschland zwischen den Haushalten und den Unternehmen aufgeteilt wird. Das Simulationsmodell belastet allein die Haushalte, ohne dadurch an Allgemeinheit zu verlieren, denn die allgemeinen Gleichgewichtswerte hängen nicht von der Aufteilung der Beitragszahlungen ab. Dieser Punkt ist wichtig - und nicht zu verwechseln mit der Höhe der Beitragssätze, denn die Höhe der Beitragssätze hat erhebliche Auswirkungen auf die Gleichgewichtswerte. Abbildung 3.4 stellt die Teile des Simulationsmodells schematisch zusammen. Links sind die exogenen Vorgaben für den Haushalts- und Produktionssektor angegeben; die entsprechenden Parameter werden zur Kalibrierung verwendet. Rechts finden sich die Vorgaben, die in der Simulation in verschiedenen Szenarien variiert werden. Abgesehen von der Fertilität und Mortalität bezeichne ich diese Parameter als Politikparameter, denn sie können durch Entscheidungen der Politik verändert werden.

Weil die Randbedingungen keine Veränderungen nach dem Zieljahr zulassen, wählt die Simulation einen Übergangszeitraum von 180 Jahren zwischen dem Start- und Zieljahr. Untersuchungen von Auerbach und Kotlikoff (1987) ergeben, daß mindestens 150 Jahre als Übergangszeitraum gewählt werden sollten. Meine eigenen Analysen zeigen, daß die Simulation in 180 Jahren genug Zeit hat, um 
einen glatten Übergang zu den Randbedingungen zu erreichen. Abschnitt 3.6.4 wird diesen Punkt verdeutlichen. Ein wesentlich kürzerer Zeitraum, zum Beispiel 70 Jahre, würde dagegen der Simulation die konstanten Randbedingungen aufzwingen, bevor das Modell konvergiert. Um diesen Fehler zu vermeiden erstreckt sich meine Simulation über 180 Jahre, obwohl ich mich bei der Auswertung der Ergebnisse auf den Zeitraum von 1996 bis 2050 beschränke.

Abbildung 3.4: Das Simulationsmodell und seine Teilmodelle

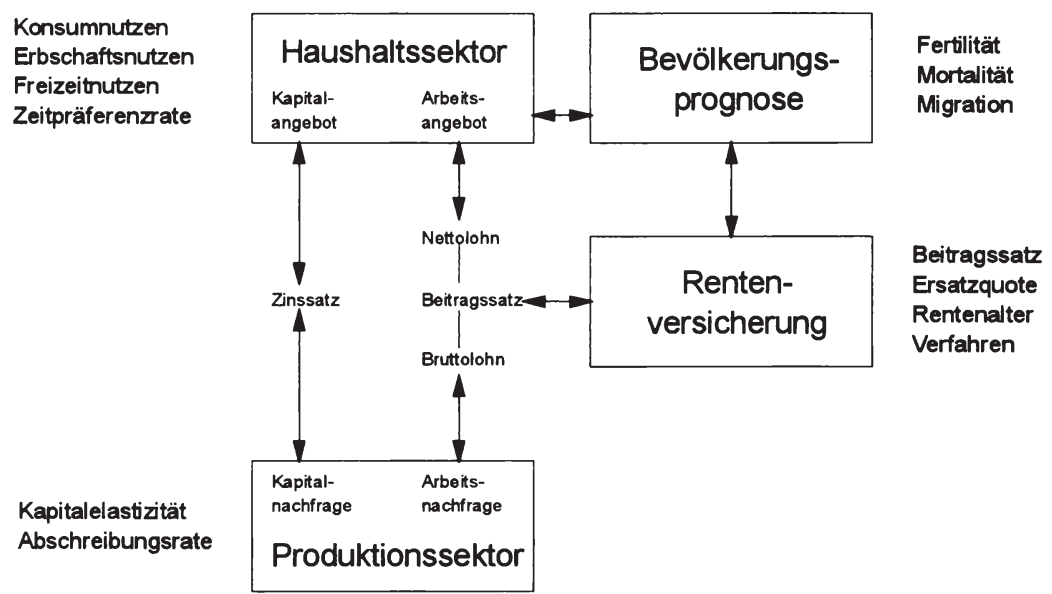

Quelle: Eigene Darstellung

Neben der Randbedingung für das Zieljahr gibt es die Randbedingung für die Situation vor dem Startjahr: Es wird ebenfalls angenommen, daß es keine Veränderungen gegeben hat. In der Realität ist diese Annahme natürlich nicht erfüllt und um diesen Fehler zumindest abzuschwächen, gebe ich der Simulation von 1960 bis 1969 zehn Jahre Zeit zum „Einschwingen“. Erst danach kalibriere ich das Simulationsmodell mit Daten aus Westdeutschland von 1970 bis 1989, dem Jahr vor der Wiedervereinigung. 
Numerische Lösung

Das Simulationsprogramm beruht auf einer nichtlinearen Fixpunktiteration, in der die einzelnen Teile des Simulationsmodells zusammengefaßt sind. Abbildung 3.5 zeigt das Schema des Simulationsprogramms, weitergehende Details bringt Anhang A2.

Abbildung 3.5: Schema des Simulationsprogramms

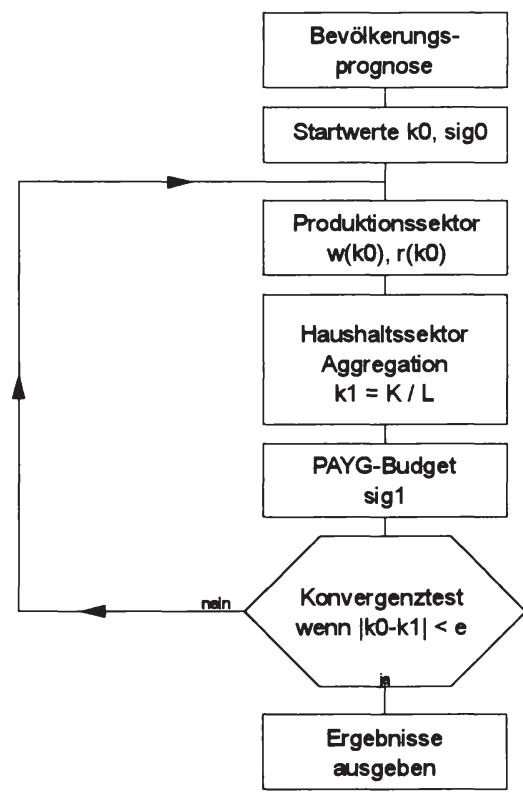

Quelle: Eigene Darstellung

Der Fixpunktiteration ist die Bevölkerungsprognose vorgeschaltet, die je nach Fertilitäts-, Mortalitäts- und Migrationsvorgaben die Bevölkerungsmatrix $n$ erstellt. Die eigentliche Iteration beginnt mit beliebigen Startwerten für die Profile der Kapitalintensität k0 und des Beitragssatzes zur Rentenversicherung sig0. Die Startwerte definieren über die Gleichungen (3.15) und (3.16) im Produktionssektor die Zinssätze und Bruttolöhne. Gleichzeitig gehen sie in das Haushaltsmodell ein, das das Arbeits- und Kapitalangebot der Kohorten über die Optimalitätsbedingungen (3.5) und (3.6) festlegt. Weil die Optimalitätsbedingungen für die meisten Nutzenfunktionen nicht analytisch aufgelöst werden können, berechnet eine im Anhang A2 beschriebene Routine die optimalen Werte numerisch. Wenn 
das Arbeits- und Kapitalangebot der Haushalte von der Arbeits- und Kapitalnachfrage der Unternehmen abweicht und die Budgetrestriktion der Rentenversicherung nicht erfültt ist, wählt der Algorithmus neue Startwerte für die Profile der Kapitalintensität und des Beitragssatzes und beginnt mit einer weiteren Iteration. Das neue Profil der Kapitalintensität entsteht aus einer Konvexkombination des alten Profils der Kapitalintensität k0 - der Nachfrage des Unternehmenssektors und dem Quotient aus Kapital- und Arbeitsangebot k1 der Haushalte. Der Algorithmus stellt sicher, daß neben dem Profil der Kapitalintensität auch das Profil des Beitragssatzes zur Rentenversicherung konvergiert.

\subsection{Kalibrierung der Simulation}

Das Simulationsmodell bestimmt Zins-, Lohn- und Beitragssätze endogen bei gegebener Bevölkerungsentwicklung und gegebenen Parametern der Produktionsund Lebensnutzenfunktion. Die exogenen Parameter der Produktions- und Lebensnutzenfunktion sollen die Verbindung zwischen dem Simulationsmodell und ausgewählten bereits realisierten Daten schaffen, also kalibrieren die exogenen Parametern das Simulationsmodell mit dem Ziel, eine möglichst hohe Übereinstimmung zwischen dem Modell und der Realität herzustellen. Die Auswahl der Daten muß sich an zwei Gesichtspunkten orientieren: erstens der Struktur des Modells und zweitens den verfügbaren Daten. Wenn das Simulationsmodell zum Beispiel den Haushaltssektor in Kohorten aufgliedert, dann sollten die endogenen Variablen des Haushaltssektors mit kohortenspezifischen Daten kalibriert werden.

Die Abschreibungsrate $\delta$ und die Kapitalelastizität $\alpha$ sind die exogenen Parameter in den Gleichungen (3.15) und (3.16), die den Produktionssektor kennzeichnen. Die Lebensnutzenfunktion (3.3) beschreibt den Haushaltssektor und enthält neben der Zeitpräferenzrate $\rho$ die Parameter der Nutzenfunktionen als exogene Größen: $\gamma$ ist Teil des Konsumnutzens (3.8), $\xi$ legt den Erbschaftsnutzen (3.9) fest und $\eta$ bestimmt den Freizeitnutzen (3.10). Insgesamt stehen damit sechs exogene Parameter zur Verfügung, mit denen das Simulationsmodell kalibriert wird. In Abbildung 3.4 lassen sich diese Parameter der linken Seite zuordnen.

Die Randbedingungen des Simulationsmodells lassen vor dem Startjahr und nach dem Zieljahr keine Veränderungen in den endogenen Variablen zu. Wie bereits erwähnt, gibt es bei einem Simulationszeitraum von 180 Jahren einen glatten Übergang zu der Zeit nach dem Zieljahr. Der Übergang von den fixierten Werten vor dem Startjahr zu den endogen bestimmten Werten nach dem Startjahr ist ebenfalls weitgehend glatt, nur in den ersten Jahren sind die Veränderungen der 
endogenen Variablen etwas größer. Weil der Algorithmus diese Veränderungen unabhängig vom Bevölkerungsprozeß verursacht, wähle ich das Jahr 1960 als Startjahr, beginne die Kalibrierung aber erst nach dem Jahr 1969. Die nächsten zwanzig Jahre von 1970 bis 1989 kalibrieren das Simulationsmodell mit Daten aus Westdeutschland

\subsubsection{Auswahl der zu kalibrierenden Variablen}

Kapitalintensität

Abbildung 3.6: Entwicklung der Kapitalelastizität zwischen 1970 und 1989

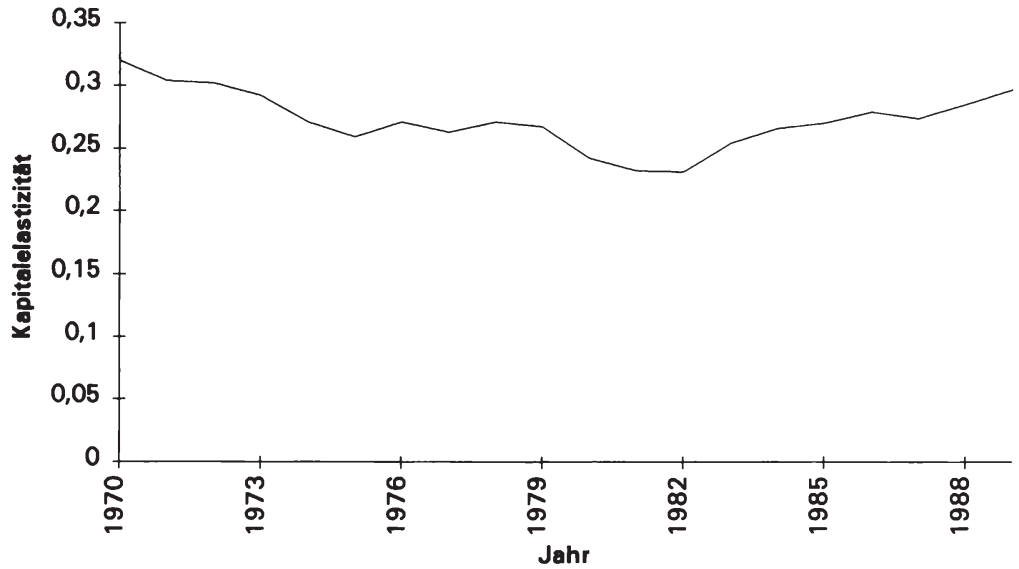

Quelle: Eigene Berechnungen auf Basis von Statistisches Bundesamt (1972b - 1992b)

Das Simulationsmodell bildet den Produktionssektor durch eine repräsentative Unternehmung ab. Diesem Aggregationsgrad entsprechen aggregierte Daten aus der volkswirtschaftlichen Gesamtrechnung. Für die Spezifikation der exogenen Parameter nutze ich zwei Eigenschaften der Cobb-Douglas Produktionsfunktion (3.13) aus: Erstens addieren sich Kapital- und Arbeitselastizität zu eins und zweitens sind Arbeitselastizität und Lohnquote gleich. Die volkswirtschaftliche Gesamtrechnung berechnet die Lohnquote als Verhältnis aus „Bruttoeinkommen aus unselbständiger Arbeit“ und „Volkseinkommen“. Abbildung 3.6 zeigt die 
Kapitalelastizitäten, die sich aus den Lohnquoten der volkswirtschaftlichen Gesamtrechnung ergeben. ${ }^{8}$

Der Mittelwert der Kapitalelastizität $\alpha$ beträgt 0,2725 , das Maximum liegt bei 0,32 im Jahr 1970 und das Minimum liegt bei 0,231 im Jahr 1982. Der Variationskoeffizient, definiert als der Quotient aus Standardabweichung und Erwartungswert, beträgt knapp 0,09 und ist damit relativ hoch. Weil die Kapitalelastizität verhältnismäßig stark schwankt, ist die Sensitivitätsanalyse in Abschnitt 3.5.3 besonders wichtig, denn sie zeigt, wie das Simulationsmodell auf Veränderungen der Kapitalelastizität reagiert. Für die Simulation setze ich die Kapitalelastizität auf den Mittelwert aus den Jahren 1970 bis 1989, also ist $\alpha=0,2725$.

Abschreibungsrate

Abbildung 3.7: Entwicklung der Abschreibungsrate zwischen 1970 und 1989

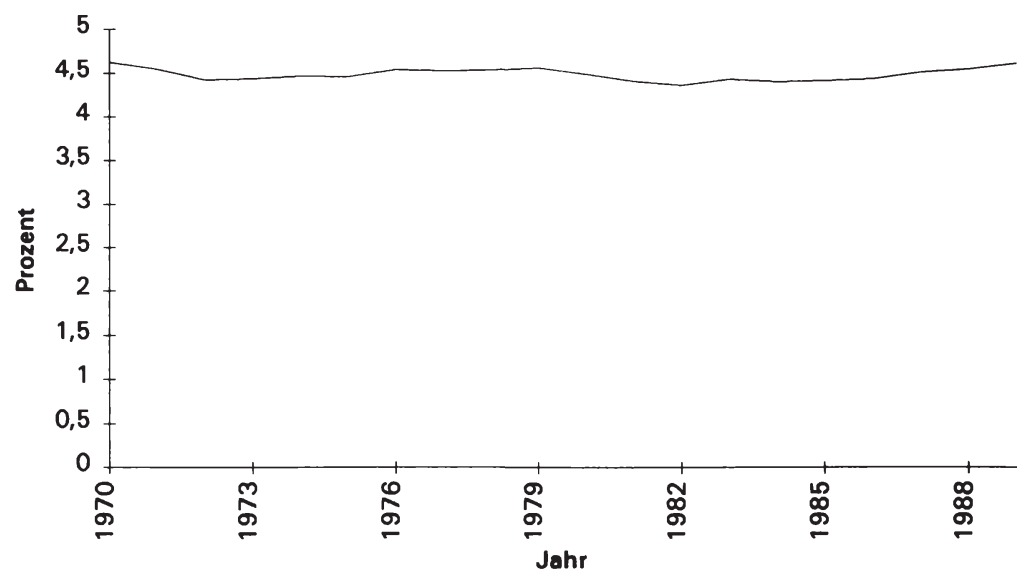

Quelle: Eigene Berechnungen auf Basis von Statistisches Bundesamt (1972b - 1992b)

8 Das „Bruttoeinkommen aus unselbständiger Arbeit“ und das „Volkseinkommen“ sind verschiedenen Jahrgängen des Statistischen Jahrbuchs entnommen, siehe Statistisches Bundesamt (1972b-1992b). 
Der zweite exogene Parameter für den Produktionssektor ist die Abschreibungsrate. Die volkswirtschaftliche Gesamtrechnung weist das „Nettoanlagevermögen zu Wiederbeschaffungspreisen am Jahresanfang" und die „Abschreibungen“" aus.9 Der Quotient aus Abschreibungen und Nettoanlagevermögen ergibt die Abschreibungsrate. Abbildung 3.7 zeigt die Entwicklung der Abschreibungsrate von 1970 bis 1989.

Die Variation der Abschreibungsrate $\delta$ ist wesentlich geringer als die Variation der Kapitalelastizität: Der Variationskoeffizient der Abschreibungsrate ist etwas geringer als 0,02 bei einem Mittelwert der Abschreibungsrate von 4,48\%. Damit ist die Abschreibungsrate statistisch gut abgesichert. In der Simulation setzte ich die Abschreibungsrate auf $\delta=0,0448$.

\section{Parameter des Haushaltssektors}

In dem Simulationsmodell besteht der Haushaltssektor aus einem repräsentativen Haushalt je Kohorte, so daß kohortenbezogene Haushaltsdaten zur Kalibrierung der Nutzenfunktion verwendet werden sollten. Die Konsumausgaben, das Einkommen, das Nettogeldvermögen ${ }^{10}$ und den Haus- und Grundbesitz nach dem Alter des Haushaltsvorstandes erfaßt die Einkommens- und Verbrauchsstichprobe (EVS), die alle fünf Jahre vom Statistischen Bundesamt durchgeführt wird. Zwischen 1970 und 1989 fanden in den Jahren 1973, 1978, 1983 und 1988 vier Erhebungen statt. Das Statistische Bundesamt veröffentlicht seine EVS-Auswertungen in den Heften 1 bis 7 der Fachserie 15'11, siehe Statistisches Bundesamt (1975a - 1990a). Aus diesen EVS-Auswertungen bilde ich drei Vergleichsgrößen, indem ich Quoten bilde. Die Quotenbildung hat den Vorteil, daß sie aus monetären Größen dimensionslose Größen erzeugt und somit unabhängig von der jeweiligen Geldeinheit ist. Die Konsumquote ist der Quotient aus monatlichen Konsumausgaben und monatlichem Haushalts-Nettoeinkommen und addiert sich mit der Sparquote zu $100 \%$. Die Vermögensquote ist definiert als das durchschnittliche Nettovermögen der Haushalte in Prozent des durchschnittlichen Jahreseinkommens. Schließlich setzt die Erwerbsquote die Anzahl der Erwerbstätigen zur Stärke einer Altersklasse in Beziehung.

9 Der öffentliche Tiefbau (z.B. Straßen, Schienenwege, Brücken) wird in der volkswirtschaftlichen Gesamtrechnung nicht abgeschrieben.

10 Das Nettogeldvermögen bereinigt das Bruttogeldvermögen um die finanziellen Restschulden.

11 Die Ergebnisse der EVS 1973 sind teilweise noch in der Reihe 18 veröffentlicht. 
Konsum und Sparquote

Abbildung 3.8 zeigt die Sparquoten aus der EVS für die Jahre 1973, 1978, 1983 und 1988 nach dem Alter des Haushaltsvorstands. Die EVS ordnet das Alter der Haushaltsvorstände relativ breiten Altersklassen zu, deren Grenzen in Abbildung 3.8 ausgewiesen sind; eine tiefere Untergliederung veröffentlicht das Statistische Bundesamt nicht. ${ }^{12}$ Die Altersklassen $65-70$ und 70++ sind in der EVS 1973 und teilweise in der EVS 1978 zur Klasse 65++ zusammengelegt, was in den folgenden Abbildungen zu berücksichtigen ist.

Abbildung 3.8: Sparquote nach dem Alter des Haushaltsvorstands in den EVS 1973, 1978, 1983 und 1988

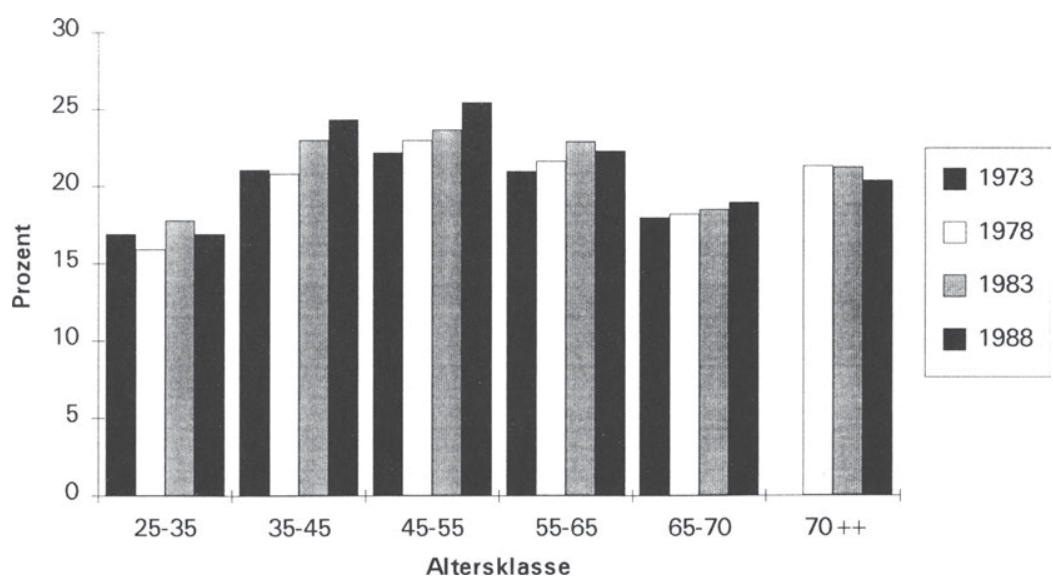

Quelle: Eigene Berechnungen auf Basis von Statistisches Bundesamt (1975a - 1992a)

Für die jüngsten Haushalte ist die Sparquote im Mittel der vier Wellen mit $17 \%$ am kleinsten. Während des Erwerbslebens steigt sie leicht an und erreicht in der Altersklasse der 45 bis 55-jährigen knapp 23,5\% im Mittel der Wellen. Bis zum Alter von 70 Jahren fällt die Sparquote wieder leicht ab, bevor sie für die über 70-jährigen wieder steigt. Weil die Sparquoten für keine Altersklasse negativ sind, nehmen die Haushalte im Kohortendurchschnitt keine Schulden auf und ha-

12 Das Statistische Bundesamt führt auf Anfrage und gegen Rechnung Sonderauswertungen der EVS durch. 
ben daher über das ganze Leben positives Vermögen. Folglich lassen die Sparquoten in Abbildung 3.8 ein Haushaltsmodell, das auf Kohortenebene ohne Liquiditätsrestriktionen auskommt, nicht als völlig abwegig erscheinen. Wenn man die Unterschiede zwischen den Erhebungsjahren betrachtet, kann man von 1973 bis 1988 für die Haushalte mittleren Alters leicht steigende Sparquoten feststellen, während die ältesten Haushalte leicht sinkende Sparquoten haben. Insgesamt weichen die Sparquoten in den einzelnen Erhebungsjahren aber nur wenig voneinander $\mathrm{ab}$. Abbildung 3.8 zeigt auch, daß der Zusammenhang zwischen dem Konsum und dem Einkommen relativ stark ist, denn die Konsumquote (100 minus Sparquote) bewegt sich zwischen $75 \%$ und $84 \%$.

\section{Vermögensquote}

Die Konstruktion der Vermögensquote erfordert einen höheren Aufwand als die Berechnung der Konsum- bzw. Sparquote. Die EVS enthält Angaben zum Nettogeldvermögen sowie zum Haus- und Grundbesitz und der darauf liegenden Restschuld der Haushalte. Das Problem liegt in der Bewertung der Immobilien: Die EVS erfaßt den Haus- und Grundbesitz nach dem steuerlichen Einheitswert, der ganz erheblich vom tatsächlichen Verkehrswert abweicht. Um das tatsächliche Vermögen der Haushalte annähernd zu quantifizieren, müssen die Immobilien folglich vom Einheitswert in den Verkehrswert umgerechnet werden. Für die EVS 1983 gibt Braun (1985) den Faktor 6,4 an, d.h. 1983 entspricht der Verkehrswert in etwa dem 6,4-fachen des ausgewiesenen Einheitswerts. Ich verwende diesen Faktor für die Umrechnung der Immobilien in der EVS 1983 und konstruiere davon ausgehend die Faktoren für die anderen Wellen. Meine identifizierende Annahme ist, daß sich das durchschnittliche Verhältnis von Verkehrswert zu Restschuld zwischen 1973, 1978 und 1983 nicht wesentlich geändert hat, denn erst nach dieser Zeit hat die Liberalisierung des Bankensektors zu geänderten Konditionen für die Haushalte geführt. Für das Jahr 1983 mit 6,4 als Faktor und dem durchschnittlichen Einheitswert von $43860 \mathrm{DM}$ beträgt der durchschnittliche Verkehrswert $280706 \mathrm{DM}$, so daß das Verhältnis von Verkehrswert zur durchschnittlichen Restschuld von 67025 DM fast 4,2 beträgt. Wenn sich die Banken bei ihrer Kreditvergabe in den Jahren 1973 und 1978 an einem Verhältnis von Verkehrswert zu Restschuld von 4,2 orientiert haben, dann lagen die Faktoren im Jahr 1973 bei 4,5 und im Jahr 1978 bei 6,0. Die gleiche Rechnung für das Jahr 1988 ergibt einen Faktor von 7,6, der mir für die Umrechnung der Einheitswerte aus der EVS 1988 zu hoch erscheint, denn mit der zunehmenden Liberalisierung der Banken führte der verschärfte Wettbewerb zu günstigeren Bedingungen für die Kreditnehmer. Statt 7,6 verwende ich daher einen Faktor von 7,0 für das Jahr 1988. 
Die Faktoren wandeln die Einheitswerte näherungsweise in die Verkehrswerte um, die vermindert um die Restschuld aus Hypothekarkrediten das Immobilienvermögen der Haushalte angeben. Die Summe aus Immobilienvermögen und Nettogeldvermögen ergibt schließlich das Nettovermögen. Natürlich ist das so konstruierte Vermögen nur ein Proxi für das tatsächliche Vermögen der Haushalte, aber nicht zuletzt wegen der grundsätzlichen Probleme bei der Erfassung des Vermögens (siehe Abschnitt 2.1.2), halte ich diesen Proxi dem Abstraktionsgrad des Simulationsmodells angemessen.

Abbildung 3.9: Vermögensquote nach dem Alter des Haushaltsvorstands in den EVS 1973, 1978, 1983 und 1988

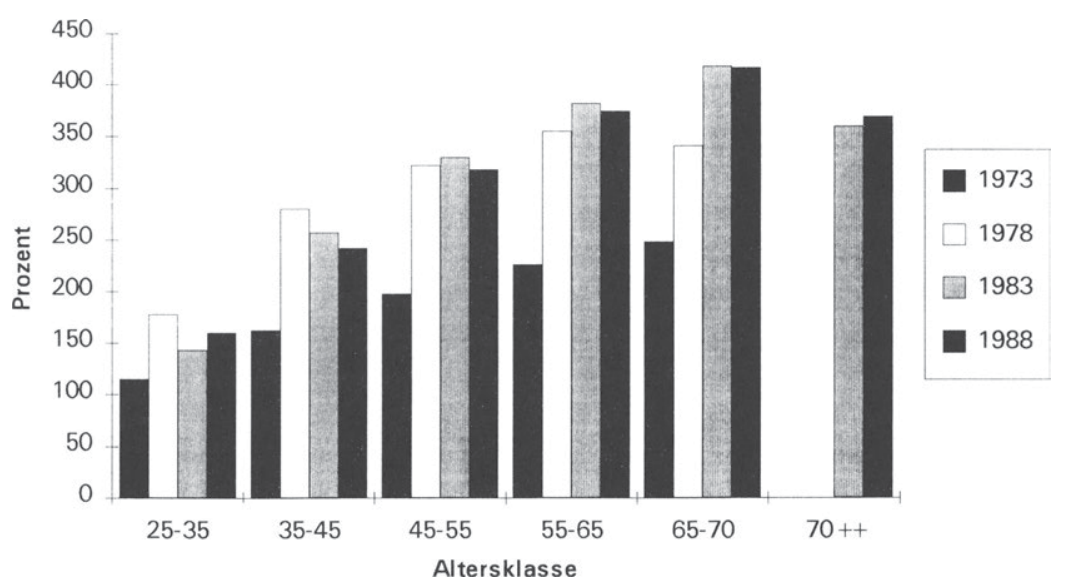

Quelle: Eigene Berechnungen auf Basis von Statistisches Bundesamt (1975a - 1992a)

Abbildung 3.9 zeigt, daß die Vermögensquoten von 1978 bis 1988 weitgehend stabil bleiben, nur in der EVS 1973 sind sie deutlich niedriger. Eine naheliegende Erklärung könnte in der Umrechnung der Einheitswerte liegen. Eine andere Erklärung könnte in unterschiedlichen Entwicklungen der Einkommenshöhe und der Vermögenswerte liegen. So hat die erste Ölkrise zu einem deutlich verlangsamten Einkommenswachstum geführt; die Entwicklung des Immobilienvermögens ist davon weniger oder gar nicht betroffen gewesen. Wenn diese Erklärung richtig ist, dann machen die niedrigen Vermögensquoten in Abbildung 3.9 durchaus Sinn. 
Klar ist, da $\beta$ die hier konstruierten Vermögensquoten nur als Proxi dienen können. Dennoch kann man einige Tendenzen erkennen: Der Vergleich zwischen den Altersklassen zeigt, daß die Vermögensquote für die Altersklasse 25-35 am niedrigsten ist und dann bis zur Altersklasse 65 - 70 ansteigt. Danach geht die Vermögensquote wieder zurück, bleibt aber deutlich über den Werten für die jungen Altersklassen. Das klassische Lebenszyklusmodell kann diesen Verlauf nur dann erklären, wenn es dem Erbschaftsmotiv ein sehr hohes Gewicht zuordnet. Abbildung 3.9 verdeutlicht erneut, daß der Verzicht auf Liquiditätsrestriktionen bei einer kohortenspezifischen Betrachtung weniger gravierend ist, denn auch für die Altersklasse 25 - 35 mit der niedrigsten Vermögensquote beträgt das Vermögen im Durchschnitt etwas mehr als ein Jahresgehalt.

Erwerbsquote

Abbildung 3.10: Erwerbsquote nach dem Alter für 1974, 1979, 1984 und 1989

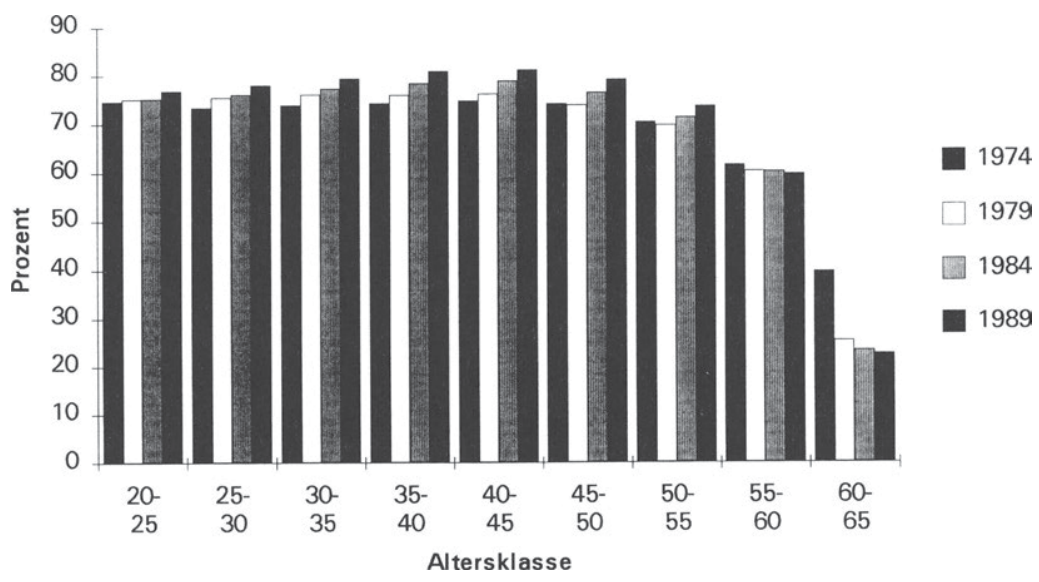

Quelle: Eigene Berechnung auf Basis von Statistisches Bundesamt (1972b - 1992b)

Weil die Erwerbsquoten für 1973 und 1988 in den Statistischen Jahrbüchern nicht ausgewiesen sind, verwende ich die Erwerbsquoten aus den Jahren 1974, 1979, 1984 und 1989 als dritte Vergleichsgröße für den Haushaltssektor. In Abbildung 3.10 ist die Erwerbsquote als die Summe der erwerbstätigen Frauen und Männer geteilt durch die Gesamtbevölkerung einer Altersklasse definiert. 
Die Altersklassen zwischen 20 und 50 Jahren unterscheiden sich nur wenig in ihrer Erwerbsbeteiligung, erst nach dem 50. Lebensjahr sinkt die Erwerbsquote deutlich. Von 1974 bis 1989 nehmen die Erwerbsquoten für die Altersklassen von 25 bis 55 Jahren leicht zu. Dieser Effekt ist eindeutig auf die höhere Erwerbsbeteiligung der Frauen zurückzuführen, die bei der getrennten Betrachtung der Erwerbsquoten von Frauen und Männern offensichtlich wird.

\section{Kapitalkoeffizient}

Die sechste Datenreihe soll schließlich den aggregierten Kapitalstock an den gesamtwirtschaftlichen Output anpassen. Aus der volkswirtschaftlichen Gesamtrechnung ergibt sich der Netto-Kapitalkoeffizient als Quotient aus Nettoanlagevermögen und Bruttoinlandsprodukt. In den zwanzig Jahren von 1970 bis 1989 ist der Netto-Kapitalkoeffizient leicht gestiegen, wie Abbildung 3.11 verdeutlicht.

Abbildung 3.11: Entwicklung des Netto-Kapitalkoeffizienten zwischen 1970 und 1989

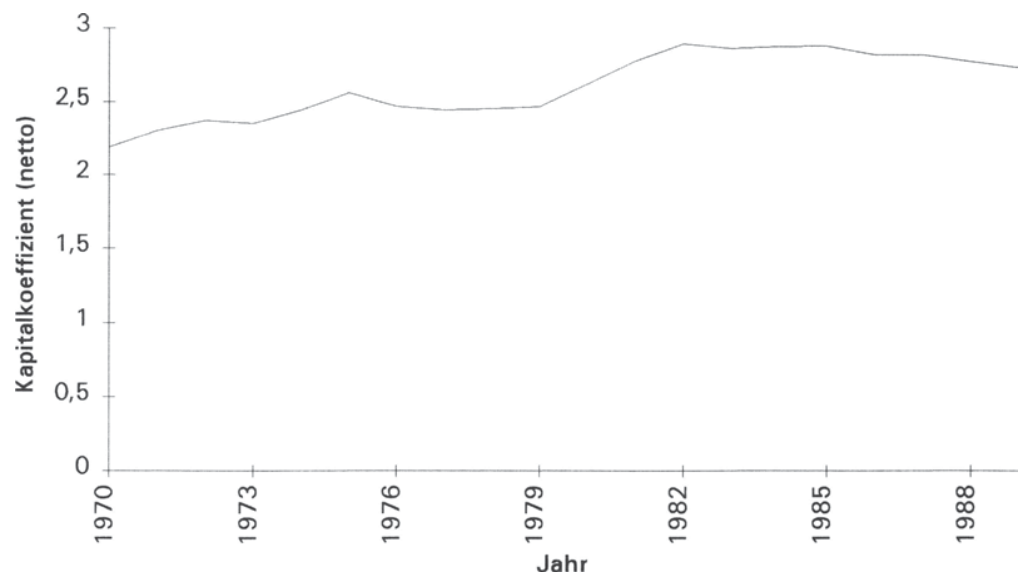

Quelle: Eigene Berechnungen auf Basis von Statistisches Bundesamt (1972b - 1992b) 


\subsubsection{Methode und Ergebnisse der Kalibrierung}

Sechs exogene Parameter bestimmen das Simulationsmodell: Die Kapitalelastizität $\alpha$, die Abschreibungsrate $\delta$ sowie die vier Parameter der Haushalte, wobei $\gamma$ (Konsum), $\xi$ (Erbschaft), $\eta$ (Freizeit) die Nutzenfunktion und $\rho$ die Zeitpräferenz festlegen. Die beiden Parameter des Produktionssektors, die Kapitalelastizität $\alpha$ und die Abschreibungsrate $\delta$, sind mit den Durchschnittswerten der Jahre 1970 bis 1989 aus der volkswirtschaftlichen Gesamtrechnung belegt. Die exogenen Parameter des Haushaltssektors paßt die Kalibrierung so an, daß die Simulationsergebnisse für die Jahre 1970 bis 1989 möglichst gut zu den Daten aus den in diesem Zeitraum erhobenen Wellen der Einkommens- und Verbrauchsstichprobe, den Erwerbsquoten und den Netto-Kapitalkoeffizienten passen.

Die Daten aus der EVS stammen aus den Jahren 1973, 1978, 1983 und 1988. Ich unternehme weder den Versuch, die Daten für die dazwischen liegenden Jahre zu konstruieren, z.B. durch einen interpolierenden Spline, noch die Altersklassen der EVS in einzelne Kohorten zu zerlegen. Statt dessen vergleiche ich die entsprechenden Ergebnisse des Simulationsmodells in den vier Jahren mit den Daten aus der EVS, indem ich die Kohorten im Simulationsmodell zu den Altersklassen der EVS zusammenfasse. Weil das Simulationsmodell auf stetigen Funktionen aufbaut, kann es weder zwischen den Variablen aufeinanderfolgender Jahre noch zwischen aufeinanderfolgenden Kohorten übermäßige Sprünge geben. Mit dem gleichen Ansatz vergleiche ich die Erwerbsquoten des Statistischen Bundesamtes mit den Ergebnissen des Simulationsmodells in den Jahren 1974, 1979, 1984 und 1989. Die vierte Zielgröße, der Netto-Kapitalkoeffizient, setzt die entsprechenden Daten aus der volkswirtschaftlichen Gesamtrechnung in allen Jahren von 1970 bis 1989 in Beziehung zu den Ergebnissen des Simulationsmodells.

Um den Unterschied zwischen den simulierten endogenen Variablen und den Daten aus den Statistiken zu messen, definiere ich eine Abstandsfunktion, die die vier Zielgrößen Konsumquote, Vermögensquote, Erwerbsquote und Netto-Kapitalkoeffizient zusammenfaßt. Die Abstandsfunktion beruht im wesentlichen auf der Idee, die Größenordnungen der Variablen vergleichbar zu machen. Dazu wird für jede Zielgröße die absolute Abweichung zwischen Simulations- und Zielgröße berechnet, die dann gleich gewichtet in Prozent der Zielgröße in die Zielfunktion eingeht.

Die Kalibrierung nimmt für die Politikparameter, die auf der rechten Seite von Abbildung 3.4 erscheinen, die entsprechenden Werte der Vergangenheit. Das durchschnittlich Verrentungsalter diskretisiere ich auf Basis der Rentenzugangsstatistik (siehe Verband Deutscher Rentenversicherungsträger, 1996); aus der gleichen Quelle entnehme ich die Netto-Ersatzquoten für den Zeitraum der Kalibrierung. Die Bevölkerungsentwicklung im Kalibrierungszeitraum entspricht der 
tatsächlichen Entwicklung, die Zahlen stammen aus den Statistischen Jahrbüchern (siehe Statistisches Bundesamt, 1962b-1992b).

Die folgende Übersicht stellt die Ergebnisse der Kalibrierung der exogenen Haushaltsparameter sowie die durch Mittelwerte bestimmten exogenen Parameter des Produktionssektors zusammen:

$\begin{array}{lll}\text { Kapitalelastizität } & \alpha & 0,2725 \\ \text { Abschreibungsrate } & \delta & 0,0448 \\ \text { Zeitpräferenzrate } & \rho & 0,035 \\ \text { Konsumnutzen } & \gamma & 3,0 \\ \text { Erbschaftsnutzen } & \xi & 10,0 \\ \text { Freizeitnutzen } & \eta & 0,19\end{array}$

Der kalibrierte Wert für die Zeitpräferenzrate $\rho$ beträgt 3,5\% und hat damit eine durchaus akzeptable Größenordnung. Offensichtlich paßt sich das allgemeine Gleichgewichtsmodell mit dem Erbschaftsnutzenmodell als Haushaltsmodell den Daten aus den offiziellen Statistiken an, ohne dabei auf unrealistische Werte für die exogenen Parameter angewiesen zu sein. Gegenüber den allgemeinen Gleichgewichtsmodellen, die das Haushaltsverhalten mit einfacheren Versionen des klassischen Lebenszyklusmodell beschreiben, ist das ein wesentlicher Fortschritt. Auerbach et al. (1989) benötigen beispielsweise eine negative Zeitpräferenzrate, um ihr Modell zu kalibrieren.

Der Parameter $\gamma$ mißt die Risikoaversion der Haushalte bezüglich der Mortalität. Der kalibrierte Parameter des Konsumnutzens hat einen Wert von $\gamma=3$. Damit liegt $\gamma$ am unteren Rand des Bereichs, den Davies (1981) in seiner oft zitierten Arbeit über den Zusammenhang zwischen unsicherer Lebenserwartung und Konsumverhalten gefunden hat:

"To sum up: The available evidence suggests that $\gamma$ may typically be above 3 ; that a value of 4 may be a "best guess" at the true figure; and that even $\gamma=5$ could not be rejected out of hand as implausible." (Davies, 1981, p. 574)

Auch spätere Arbeiten von Kotlikoff et al. $(1986,1987)$ und Hubbart (1987) verwenden im Zusammenhang mit einer unsichereren Lebenserwartung Werte für die Mortalitäts-Risikoaversion $\gamma$, die in dem von Davies angegebenen Bereich liegen.

Die Erbschaftsnutzenfunktion und ihr Parameter $\xi$ sind bisher nicht in allgemeinen Gleichgewichtsmodellen verwendet worden, so daß der kalibrierte Wert $\xi=10$ nicht in die Literatur eingeordnet werden kann. Um dennoch eine Interpretation des Erbschaftsparameters zu ermöglichen, erinnere ich an die Diskussion in Abschnitt 2.4: Dort habe ich die marginale Rate der Substitution zwischen 
Konsum und Vermögen (MRSA) eingeführt, mit der die marginale Zahlungsbereitschaft beim Tausch von Konsumeinheiten in Jahr $j$ - 1 gegen Vermögenseinheiten in Jahr $j$ gemessen wird. Weil sich die MRSA über den Lebenszyklus ändert, läßt sich die Bedeutung des Erbschaftsnutzens (und damit des Parameters $\xi$ ) am besten mit einem Beispielprofil verdeutlichen. Ich wähle die 1934 geborene Kohorte als Beispiel. Mit den kalibrierten Werten der exogenen Parameter ist die MRSA im 22. Lebensjahr dieser Kohorte mit 0,0026 im Vergleich zur MRSC von 0,8755 sehr klein. Im 50. Lebensjahr ist die MRSA wegen des inzwischen höheren Vermögens noch kleiner: Sie beträgt 0,0008 im Vergleich zur MRSC von 0,9464 . Erst spät, mit der steigenden Sterbewahrscheinlichkeit, steigt die MRSA, aber auch im Alter von 90 Jahren erreicht sie lediglich den Wert von 0,1215 und ist immer noch deutlich kleiner als die MRSC von 0,8115. In Relation zum Konsumnutzen hat der Erbschaftsnutzen also kein übermäßig hohes Gewicht. Auch das ist ein Fortschritt gegenüber den Arbeiten von Auerbach et al. (1989) sowie Raffelhüschen und Kitterer (1990), in denen das Erbschaftsmotiv bei sicherer Lebenserwartung nur am Lebensende Nutzen stiftet und ein sehr hohes Gewicht hat.

Den Parameter des Freizeitnutzens interpretiere ich ebenfalls mit Hilfe der Beispielkohorte, die 1934 geboren wurde. Für diese Kohorte steigt die Lohnelastizität des Arbeitsangebotes mit dem Lebensalter leicht an und beträgt für den kalibrierten Parameter $\eta=0,19$ im Mittel der Erwerbsjahre knapp 0,32. Dieser Wert liegt im Bereich der geschätzten Lohnelastizitäten, die Börsch-Supan (1995a) aus verschiedenen Studien zusammengestellt hat.

Weil die logarithmische Freizeitnutzenfunktion (3.10) ebenfalls zur Familie der isoelastischen Nutzenfunktionen gehört, ergibt sich ein interessanter Vergleich: Die logarithmische Nutzenfunktion ist der Grenzfall der isoelastischen Nutzenfunktion wenn $\gamma$ gegen eins geht. Der kalibrierte Wert für $\gamma$ ist drei, also ist die Krümmung der Konsumnutzenfunktion wesentlich stärker als die Krümmung der Freizeitnutzenfunktion. Die ökonomische Interpretation dieser mathematischen Eigenschaft sagt, da $B$ den Leuten die Konsumglättung wichtiger als die Freizeitglättung ist.

\subsubsection{Sensitivitätsanalyse}

Bevor ich das Simulationsmodell mit den kalibrierten exogenen Parametern anwende und verschiedene Szenarien durchspiele, untersuche ich die Sensitivität der endogenen Variablen auf Variationen der exogenen Parameter. Diese Analyse zeigt, wie stark die Ergebnisse der Simulation von den exogenen Parametern abhängen. Wenn die Abhängigkeit gering ist, dann kann man den Ergebnissen der Simulation eher vertrauen als bei einer großen Sensitivität. Die Sensitivität der 
endogenen Variablen messe ich mit der Elastizität der endogenen Variablen bezüglich einer Änderung der exogenen Variablen. Dazu gebe ich ein Beispiel: Betrachtet wird die Änderung des Kapitalstocks $K$ nach einer Änderung der Kapitalelastizität $\alpha$. Da die entsprechenden Ableitungen analytisch nicht zu berechnen sind, approximiere ich sie durch die Differenzenquotienten:

$$
\Delta K=\frac{K(\alpha+0,01 \alpha)-K(x)}{0,01 \alpha} \frac{\alpha}{K}=\frac{K(1,01 \alpha)-K(\alpha)}{K(\alpha)} \cdot 100
$$

Eine Elastizität, deren Betrag eins überschreitet, bedeutet, daß sich die endogene Variable prozentual stärker ändert als der exogene Parameter. Die Elastizitäten als Ergebnis der Sensitivitätsanalyse sind in Tabelle 3.4 für die wichtigsten endogenen Variablen im Jahr 2040 zusammengestellt.

Tabelle 3.4: Sensitivität von endogenen Variablen im Jahr 2040

\begin{tabular}{|l||r|r|r|r|r|r|}
\hline \multicolumn{1}{|c||}{ exogener Parameter } & $\Delta K$ & $\Delta L$ & $\Delta w^{b}$ & $\Delta r$ & $\Delta s$ & $\Delta \sigma$ \\
\hline \hline Kapitalelastizität $\alpha$ & 1,30 & $-0,23$ & 0,43 & 0,50 & 0,71 & $*$ \\
\hline Abschreibungsrate $\delta$ & $-0,43$ & 0,08 & $-0,14$ & $-0,16$ & 0,99 & $*$ \\
\hline Zeitpräferenzrate $\rho$ & $-0,42$ & 0,02 & $-0,12$ & 0,59 & $-0,30$ & $-0,01$ \\
\hline Konsumparameter $\gamma$ & $-0,07$ & $-0,11$ & 0,01 & $-0,05$ & $-0,02$ & $-0,03$ \\
\hline Vermögensparameter $\xi$ & 0,03 & $*$ & 0,01 & $-0,04$ & 0,03 & $*$ \\
\hline Freizeitparameter $\eta$ & $-0,12$ & $-0,15$ & 0,01 & $-0,04$ & 0,04 & $*$ \\
\hline
\end{tabular}

Anmerkungen:Elastizitäten unter 0,01 sind durch * gekennzeichnet.

In jeder Spalte ist die Elastizität mit dem größten Betrag schattiert.

Quelle:

Eigene Berechnungen

In Tabelle 3.4 ist nur eine Elastizität größer als eins. Nicht überraschend reagiert das aggregierte Kapital sehr stark auf Änderungen der Kapitalelastizität $\alpha$ - die entsprechende Elastizität von 1,3 ist die betragsmäßig größte Elastizität. Fast eins ist die Elastizität mit der die Reaktion der Sparquote auf eine Änderung der Abschreibungsrate gemessen wird. Alle anderen Elastizitäten sind deutlich kleiner als eins, so daß die endogenen Variablen insgesamt relativ wenig auf Änderungen der exogenen Parameter reagieren. Interessant ist die Frage, welche exogenen Parameter die größten Veränderungen der endogenen Variablen herbeiführen. Tabelle 3.4 hebt das Maximum jeder Spalte schattiert hervor und macht deutlich, daß die Kapitalelastizität $\alpha$ für drei der endogenen Variablen an erster Stelle und 
für zwei an zweiter Stelle steht. Mit einem deutlichen Abstand folgen die Zeitpräferenz- und Abschreibungsrate. Am wenigsten beeinflußt der Erbschaftsnutzen die endogenen Variablen: Sein Parameter $\xi$ weist durchweg geringe Elastizitäten auf. Das ist ein wichtiges Ergebnis, denn der Erbschaftsnutzen ist der Teil des Simulationsmodells, der empirisch am wenigsten abgesichert ist. Die geringen Elastizitäten bedeuten aber nicht, daß der Erbschaftsnutzen unwichtig ist, vielmehr gilt das Gegenteil: Durch den Erbschaftsnutzen bei unsicherer Lebenserwartung erhält das Haushaltsmodell genügend Flexibilität, um sich den Daten mit exogenen Parametern in realistischen Größenordnungen anzupassen.

\subsubsection{Konvergenz zum Steady-State}

Ein letzter Test vor der Anwendung des Simulationsmodells beschäftigt sich mit der Konvergenz der Modellökonomie zum Steady-State, denn letztlich sollte das allgemeine Gleichgewichtsmodell zu einem glatten Übergang in einen langfristig stabilen Zustand der Volkswirtschaft führen. Ursache für die kurz- und mittelfristigen Störungen ist der demographische Wandel, also kann sich ein neues Gleichgewicht erst dann einstellen, wenn die Bevölkerung eine neue, stabile Verteilung erreicht. Dieser Prozeß wird einige Generationen dauern.

Ein Steady-State ist durch eine stabile Kapitalintensität gekennzeichnet, d.h. das Verhältnis von Produktionskapital zu Arbeitnehmern bleibt konstant, was von Jahr zu Jahr konstante Zinssätze und Löhne sowie konstanten Output pro Arbeitnehmer impliziert. Folglich muß im Steady-State die prozentuale Veränderung der Kapitalintensität null sein. Gleichung (3.23) definiert die prozentuale Veränderung $\Delta x$ zwischen den Jahren $j$ und $j+1$, wobei $x$ die Kapitalintensität bezeichnet.

$$
\Delta x_{j}=\frac{x_{1+1}-x_{j}}{x_{J}} \cdot 100
$$

Wenn die prozentuale Veränderung der Kapitalintensität im Simulationsmodell mit den Jahren gegen null geht, dann konvergiert die Simulation langfristig gegen einen neuen Steady-State. Abbildung 3.12 zeigt, wie die prozentuale Veränderung der Kapitalintensität bei einem Simulationszeitraum von 300 Jahren verläuft.

Abbildung 3.12 offenbart zwei Eigenschaften von $\Delta x$ : Erstens nehmen die Beträge der Maxima und Minima mit den Jahren ab, also konvergiert die Simulation langfristig zu einem neuen Steady-State. Zweitens beträgt der Abstand sowohl zwischen zwei benachbarten Maxima als auch zwischen zwei benachbarten Minima etwa 30 Jahre. Weil die Generationen mit ungefähr diesem Abstand aufeinander folgen wird deutlich, daß der demographische Wandel die entscheidende "Störgröße“ des Modells ist. Der starke Rückgang zwischen den Jahren 2021 und 
2033 tritt ein, wenn die Babyboom-Generation in Rente geht. Mit der gleichen Erklärung - Erwerbsbeginn und Verrentung - ergibt sich eine Verbindung zwischen den Kindern und Enkeln der Babyboom-Generation auf der einen Seite und den späteren Maxima und Minima auf der anderen Seite. Die Ausschläge werden jedoch zunehmend schwächer, weil jede Kindergeneration einen größeren Zeitraum als die Elterngeneration umfaßt. Die demographische Entwicklung verteilt den „Buckel“ von Generation zu Generation über einen größeren Bereich.

Abbildung 3.12: Prozentuale Veränderung der Kapitalintensität

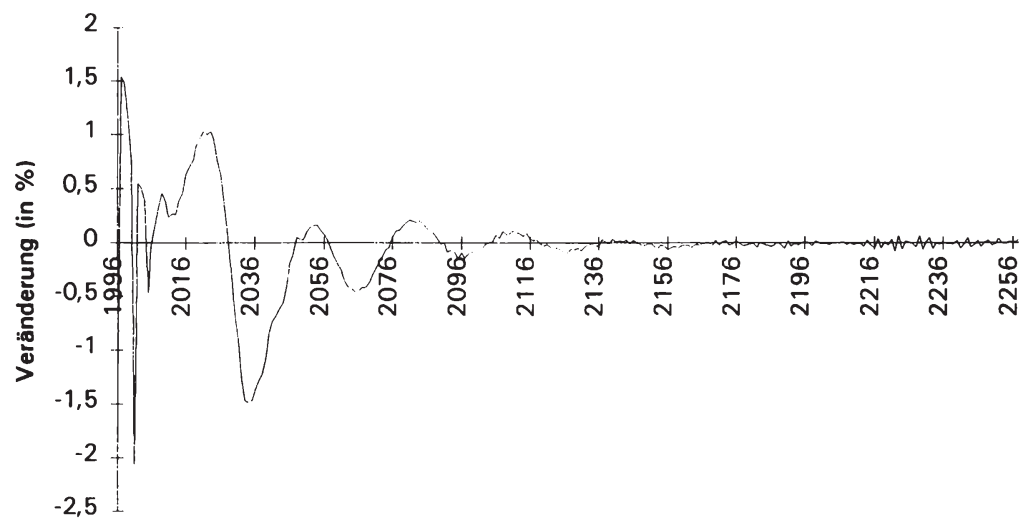

Jahr

Quelle: Eigene Berechnungen

Das in Abbildung 3.12 gezeigte Konvergenzverhalten gibt die Begründung für die Wahl des Simulationszeitraumes von 180 Jahren, der dem Zeitraum von 1960 bis 2139 entspricht. Nach dem Jahr 2135 findet praktisch keine Veränderung in der Kapitalintensität mehr statt, also ist die Endbedingung, durch die die Kapitalintensität und damit die Zinssätze und Löhne nach dem Zieljahr auf konstante Werte gesetzt werden, keine einschneidende Restriktion. Die festgesetzte Kapitalintensität ist folglich auch nach dem Zieljahr vereinbar mit einem allgemeinen Gleichgewicht, d.h. Angebot und Nachfrage auf den Märkten stimmen überein. Bei einem deutlich kürzeren Simulationszeitraum, beispielsweise 100 Jahre, wäre die dann schon früh auf einen festen Wert gezwungene Kapitalintensität nur im Zieljahr mit einem allgemeinen Gleichgewicht vereinbar. Nach dem Zieljahr würden Angebot und Nachfrage auf den Märkten nicht mehr übereinstimmen. Um 
diesen Fehler auszuschließen ist die Analyse des Konvergenzverhaltens eine wichtige Vorarbeit einer Simulation, ebenso wie die Kalibrierung und die Sensitivitätsanalyse. 
Matthias Meier - 978-3-631-75559-4

Downloaded from PubFactory at 01/11/2019 03:34:56AM

via free access 


\section{Ergebnisse des Simulationsmodells}

Nachdem die vorhergehenden Kapitel die Aufbauarbeit geleistet haben, wende ich das Simulationsmodell nun mit dem Ziel an, die makroökonomischen Auswirkungen des demographischen Wandels bei verschiedenen Reformen des Rentensystems zu untersuchen. Breyer (1996) unterscheidet fünf grundsätzliche Optionen, mit denen die Politik auf die demographischen Veränderungen und die dadurch ausgelösten Probleme der Rentenfinanzierung reagieren kann. Ich gebe Breyers Abgrenzung wieder:

A. Beibehaltung des Umlageverfahrens:

Die Beitragserhebung erfolgt weiterhin als Prozentsatz vom Arbeitseinkommen, und es gilt weiterhin das Prinzip der Teilhabeäquivalenz, nach dem innerhalb einer Alterskohorte das Verhältnis der Rentenansprüche dem der geleisteten Beiträge entspricht. [...]

B. Anpassung der Regeln innerhalb des Umlageverfahrens:

Das Umlageprinzip wird beibehalten, es werden jedoch Maßnahmen ergriffen, die geeignet sind, das Verhältnis zwischen Rentenniveau und Beitragssatz zu verbessern, z.B. eine Verlängerung der Lebensarbeitszeit oder Anreize zu einer Erhöhung der Erwerbsbeteiligung.

C. Aktive Einwanderungspolitik:

Das Umlageverfahren wird beibehalten und die Lücken in der Alterspyramide der deutschen Bevölkerung werden durch eine gezielte Zuwanderungspolitik ausgeglichen.

D. Abschaffung des Umlageverfahrens:

Die Rentenansprüche nach dem Umlageverfahren werden schrittweise abgebaut und ersetzt durch ein staatliches Kapitaldeckungsverfahren, ein privates obligatorisches Kapitaldeckungsverfahren oder eine freiwillige private Altersvorsorge. [...]

E. Mischsystem:

Die reine Umlagefinanzierung wird durch ein System ersetzt, bei dem nur noch eine für alle Bürger gleiche Grundrente nach dem Umlageverfahren finanziert wird, während eine Zusatzversorgung freiwillig (und damit nach dem Kapitaldeckungsverfahren) erfolgt. (Breyer, 1996, S. 61-62)

Ausgehend von Breyers Abgrenzung untersuche ich ebenfalls fünf unterschiedliche Szenarien, um so die grundsätzlichen Optionen der Politik zu untersuchen. Dazu beginnt Abschnitt 4.1 mit einer ausführlichen Darstellung des Basisszenarios, das auf der Basisvariante der Bevölkerungsprognose aufbaut und die heute gültigen Regelungen fortschreibt. Dieses Szenario entspricht in Breyers Abgren- 
zung der Option A. Um den Einfluß der eher politikfernen Parameter Fertilität und Mortalität einzugrenzen, vergleicht Abschnitt 4.1 das Basisszenario mit zwei Szenarien, in denen diese Parameter gegenüber dem Basisszenario verändert werden.

Nach der Diskussion der makroökonomischen Entwicklung im Basisszenario setzt Abschnitt 4.2 vier Politikszenarien, die die Optionen B bis E aus Breyers Abgrenzung aufnehmen, in Bezug zum Basisszenario. Der Vergleich konzentriert sich auf die Entwicklung der wichtigsten endogenen Variablen zwischen den Jahren 1996 und 2050: Kapitalintensität, Sparquote, Beitragssatz zur Rentenversicherung, Erwerbsstundenquote und Pro-Kopf-Konsum. Abschnitt 4.3 stellt der geschlossenen Volkswirtschaft eine kleine offene Volkswirtschaft gegenüber und zeigt, wie sich das Basisszenario und eines der Politikszenarien bei ungehindertem Kapitalverkehr entwickeln.

\subsection{Das Basisszenario}

Das Basisszenario geht davon aus, daß die derzeit gültigen Regelungen auch in der Zukunft bestehen bleiben. Konkret bedeutet das ein Verrentungsalter von 59 Jahren ${ }^{1}$ bis zum Jahr 1996, von 60 Jahren bis zum Jahr 2001 und von 61 Jahren nach dem Jahr 2001, eine Netto-Ersatzquote von $72 \%$ des durchschnittlichen Netto-Lebensarbeitseinkommens und eine Zuwanderung von 200.000 Migranten pro Jahr ab dem Jahr 2000. Jährliche Anpassungen des Beitragssatzes setzen die Budgetrestriktion der umlagefinanzierten Rentenversicherung durch. Die Bevölkerungsprognose geht von der in Abschnitt 3.2 definierten Basisvariante aus, die eine konstante Nettoreproduktionsrate von 1,39, eine weiter steigende Lebenserwartung Neugeborener und einen konstanten Migrationssaldo von jährlich 200.000 Einwanderern nach dem Jahr 2000 vorsieht.

Dem Basisszenario werden die beiden Szenarien ,konstante Mortalität“ und „steigende Fertilität" gegenübergestellt. „Konstante Mortalität“ geht gemäß der achten koordinierten Bevölkerungsvorausberechnung des Statistischen Bundesamtes, siehe Sommer (1994), von einer bis zum Jahr 2000 um 1,5 Jahre steigenden Lebenserwartung aus, die ab dem Jahr 2000 konstant bleibt. „Steigende Fertilität" untersucht eine allmählich ansteigende Nettoreproduktionsrate, die von 1,39 im Jahr 1990 über 1,45 im Jahr 2010 und 1,51 im Jahr 2030 schließlich 1,57 im Jahr 2050 erreicht.

1 Ein Verrentungsalter von 59 Jahren bedeutet für das Simulationsmodell, daß die Leute nach der Vollendung ihres 59. Lebensjahres in Rente gehen. 
Die gesamtwirtschaftliche Entwicklung wird entscheidend durch die Kapitalintensität geprägt, denn Output, Zinssatz und Bruttolohn sind eine Funktion der Kapitalintensität (siehe Abschnitt 3.3). Die Kapitalintensität ist definiert als das durchschnittliche Kapital pro Arbeitnehmer, also wird sie direkt durch die Bevölkerungsentwicklung beeinflußt. Offensichtlich ist, daß eine zurückgehende Zahl von Arbeitnehmern ceteris paribus die Kapitalintensität erhöht. Aber auch das gesamtwirtschaftliche Kapitalangebot kann von der Bevölkerungsentwicklung abhängen - wenn sich die Sparquoten der Haushalte während des Lebens ändern. Abbildung 4.1 zeigt die Entwicklung der Kapitalintensität relativ zum Indexjahr 1996. Die Indexbildung ist sinnvoll, weil das Kapital in Geldeinheiten gemessen wird, die nicht kalibriert worden sind.

Abbildung 4.1: Entwicklung der Kapitalintensität (Index $1996=1$ )

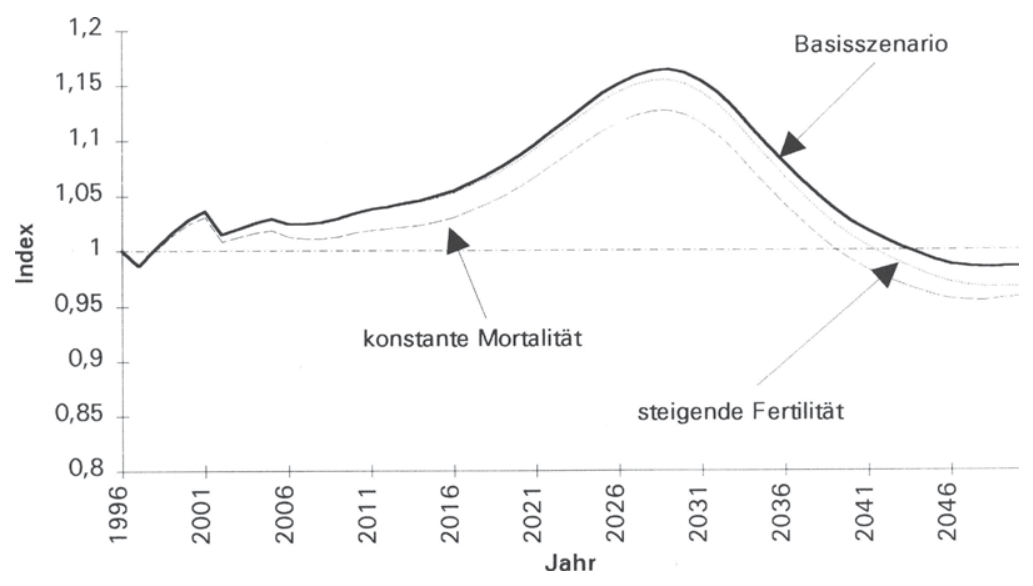

Quelle: Eigene Berechnungen

Sowohl im Basisszenario als auch in den Szenarien „konstante Mortalität“ und „steigende Fertilität“ nimmt die Kapitalintensität einen ähnlichen Verlauf. Die auffälligen Störungen zu Beginn des abgebildeten Zeitraums sind ein Artefakt und haben ihre Ursache in der Erhöhung des Verrentungsalters von 59 auf $60 \mathrm{im}$ Jahr 1996 und von 60 auf $61 \mathrm{im}$ Jahr 2001. Nach den Anfangsstörungen steigt die Kapitalintensität in allen drei Szenarien bis zu ihrem Maximum im Jahr 2029 an. Der starke Anstieg nach dem Jahr 2016 wird durch die Verrentung der Babyboom-Generation ausgelöst, die bis zum Jahr 2029 aus dem Erwerbsleben ausge- 
schieden ist. Bis zu diesem Zeitpunkt bestimmt die abnehmende Zahl der Erwerbstätigen die Entwicklung der Kapitalintensität. Erst danach, wenn die schwach besetzten „Pillenknick“-Jahrgänge in den Ruhestand wechseln, überwiegt die sinkende private Ersparnis den Rückgang der Erwerbstätigkeit, so daß die Kapitalintensität ebenfalls sinkt und unter den Ausgangswert von 1996 fällt. „Konstante Mortalität" liegt ab dem Jahr 2040 unter dem Ausgangswert, „steigende Fertilität“ ab dem Jahr 2042 und das Basisszenario ab dem Jahr 2044. Im Szenario „konstante Mortalität" ist die Kapitalintensität im Vergleich zu den anderen Szenarien am niedrigsten, denn das aggregierte Kapitalangebot ist im Szenario „konstante Mortalität“ am geringsten: Wegen der geringeren Lebenserwartung ist der Ruhestand bei konstantem Verrentungsalter kürzer, also wird ein kleineres Vermögen zur Aufbesserung der Rente benötigt. Die im Vergleich zum Basisszenario zunehmend geringer werdende Kapitalintensität im Szenario „steigende Fertilität" wird dagegen durch die steigende Zahl der Erwerbspersonen erklärt. Dieser Effekt braucht allerdings Zeit, denn zwischen der Geburt und dem Eintritt in das Erwerbsleben liegt die „unproduktive“ Kindheit. Veränderungen am Fundament des Bevölkerungsaufbaus haben daher sehr langfristige Effekte; schneller schlagen sich Änderungen am Dach des Bevölkerungsaufbaus nieder. Die schon früh erkennbare Abweichung der Kapitalintensitäten zwischen dem Basisszenario und dem Szenario „konstante Mortalität“ belegt diese Feststellung genauso wie die nur sehr langsam zunehmende Abweichung der Kapitalintensitäten zwischen dem Basisszenario und dem Szenario „steigende Fertilität".

Parallel zur Entwicklung der Kapitalintensität verläuft der Output pro Arbeitnehmer, der deshalb nicht in einer eigenen Abbildung dargestellt ist. Auch der Zinssatz ist mit der Kapitalintensität korreliert, im Gegensatz zum Output und Lohn allerdings negativ. Abbildung 4.2 zeigt die Entwicklung der Sparquote. Zusätzlich ist die Entwicklung des Zinssatzes im Basisszenario abgebildet, denn die Frage, ob und wie hoch der Zinssatz und die Ersparnis korreliert sind, ist immer noch nicht eindeutig geklärt. Die Ursache dieser Kontroverse liegt in den Einkommensund Substitutionseffekten, die durch Zinsänderungen ausgelöst werden. Weil sich der beobachtbare Gesamteffekt aus diesen beiden Teileffekten zusammensetzt, ist das Vorzeichen des Gesamteffekts umstritten, siehe Boskin (1978), Summers (1981), Bosworth et al. (1991) sowie Owens (1993). In einer Schätzung der gesamtwirtschaftlichen Konsumfunktion für Westdeutschland berechnet die Deutsche Bundesbank (1996) eine Zinselastizität des privaten Verbrauchs von -0,15. Demnach bedeutet ein Anstieg der Zinsen einen leichten Rückgang des Konsums zugunsten eines leichten Anstiegs der Ersparnis. 
Abbildung 4.2: Entwicklung der Sparquote und des Zinssatzes

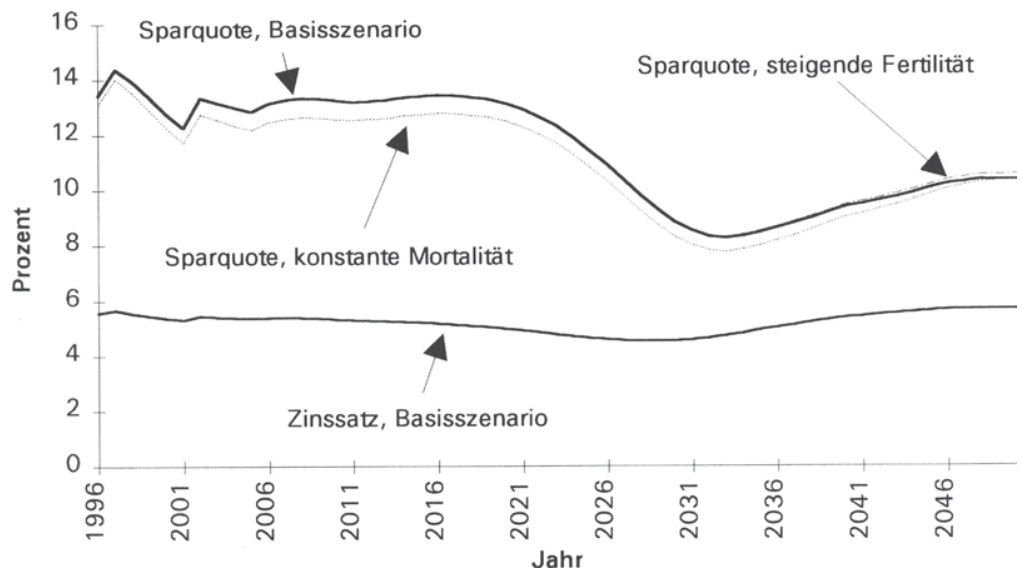

Quelle: Eigene Berechnungen

Ausgehend von 5,6\% im Jahr 1996 fällt der Zinssatz bis zum Jahr 2029 auf 4,5\%, um dann wieder auf Werte um 5,7\% anzusteigen. Die Sparquote beginnt im Basisszenario im Jahr 1996 bei 13,4 \%, erreicht im Jahr 2033 nach der Verrentung der Babyboom-Generation ein Minimum von $8,3 \%$ und steigt bis zum Jahr 2050 wieder auf $10,3 \%$ an. Fast identisch verläuft die Sparquote im Szenario „steigende Fertilität“, denn bis die höheren Geburtenraten zu einer höheren Anzahl von Haushalten im sparfähigen Alter führt, vergehen in meiner Simulation 47 Jahre. Denn erst wenn die Kinder den elterlichen Haushalt verlassen haben, beginnen die Eltern nennenswerte Beträge zu sparen. So wird verständlich, warum erst am Ende des gezeigten Zeitraums eine leichte Zunahme der Sparquote im Szenario „steigende Fertilität“ erkennbar wird. Dagegen liegt die Sparquote im Szenario „konstante Mortalität" während des gesamten Zeitraums unter der Sparquote des Basisszenarios. Dieses Ergebnis überrascht nicht, denn die niedrigere Kapitalintensität im Szenario „konstante Mortalität“ wird ja gerade durch das geringere private Kapitalangebot verursacht.

Gegenüber der Simulation von Auerbach et al. (1989) entwickelt sich die Sparquote in meiner Simulation weit weniger dramatisch. Bei Auerbach et al. sinkt die Sparquote von ca. $20 \%$ im Jahr 1995 auf weniger als $3 \%$ im Jahr 2050, obwohl die Zeitpräferenzrate mit $-5,5 \%$ in ihrer Simulation eine starke Zukunftsorientie- 
rung der Haushalte unterstellt. In Abbildung 4.2 bewegt sich die Sparquote im Basisszenario zwischen $15 \%$ und $8 \%$. Auch der Verlauf ist bei Auerbach et al. anders, dort fällt die Sparquote kontinuierlich, es gibt keine Trendumkehr nach dem Jahr 2030. Dagegen prognostizieren Cutler et al. (1990) sowie Börsch-Supan (1995b) einen nicht vom Niveau, aber vom Profil her ähnlichen Verlauf der Sparquote, obwohl ihre Modelle eine andere Struktur haben als meine Simulation, die sich an Auerbach et al. orientiert.

Abbildung 4.2 zeigt einen leicht positiven Zusammenhang zwischen Sparquote und Zinssatz, der auch in dem positiven Korrelationskoeffizienten von 0,19 deutlich wird. Dies paßt zum Ergebnis der Deutschen Bundesbank (1996), die eine positive Zinselastizität der Ersparnisse schätzt. Im Vergleich zur Ausgangssituation im Jahr 1996 behält der Zinssatz langfristig sein Niveau, während die Sparquote um gut zwanzig Prozent sinkt. Die im Vergleich zum Zinssatz sehr viel stärkere Veränderung der Sparquote in Abbildung 4.2 zeigt, daß die demographische Entwicklung einen stärkeren Einfluß auf die Sparquote als auf den Zinssatz hat. Wie kann man diese Beobachtung erklären? Weil der Zinssatz von der Kapitalintensität abhängt, diese aber nicht nur von der zurückgehenden Ersparnis, sondern auch von der abnehmenden Erwerbsbevölkerung bestimmt wird, sind die Auswirkungen der Bevölkerungsentwicklung auf die Kapitalintensität und den Output pro Arbeitnehmer sehr viel geringer als ein erster Blick auf die Sparquote vermuten läßt. Die Sparquote schwankt gerade deshalb so deutlich, weil sie die Veränderungen der Erwerbsbevölkerung teilweise auffängt und so eine gleichmäßigere Entwicklung der Kapitalintensität ermöglicht. Abbildung 4.2 belegt diesen Schluß und macht deutlich, wie wichtig ein allgemeines Gleichgewichtsmodell für die Untersuchung des demographischen Wandels ist.

Die Sparquote ist Ausdruck der Vermögensakkumulation und steht daher auch in einem Zusammenhang mit den Erbschaften. In den Arbeiten von Auerbach et al. (1989); Raffelhüschen und Kitterer (1990) sowie Kraus und Buslei (1996) erreichen alle Haushalte mit Sicherheit das maximale Lebensalter und hinterlassen ihren Kindern am Ende des maximalen Lebensalters ihr noch vorhandenes Vermögen. Also gibt es im Lebenszyklus der Haushalte nur einen Zeitpunkt, in dem Erbschaften empfangen werden. Auf individueller Ebene ist das vielleicht plausibel, nicht aber auf Kohortenebene. Wegen der unsicheren Lebenserwartung verteilen sich die empfangenen Erbschaften in meinem Simulationsmodell für den durchschnittlichen Haushalt einer Kohorte über einen großen Altersbereich, was gegenüber der Konzentration auf einen einzigen Zeitpunkt sehr viel realistischer ist. Abbildung 4.3 zeigt zur Verdeutlichung für zwei ausgewählte Kohorten die durchschnittlich erhaltenen Erbschaften im Basisszenario. 
Abbildung 4.3: Durchschnittlich erhaltene Erbschaften im Basisszenario

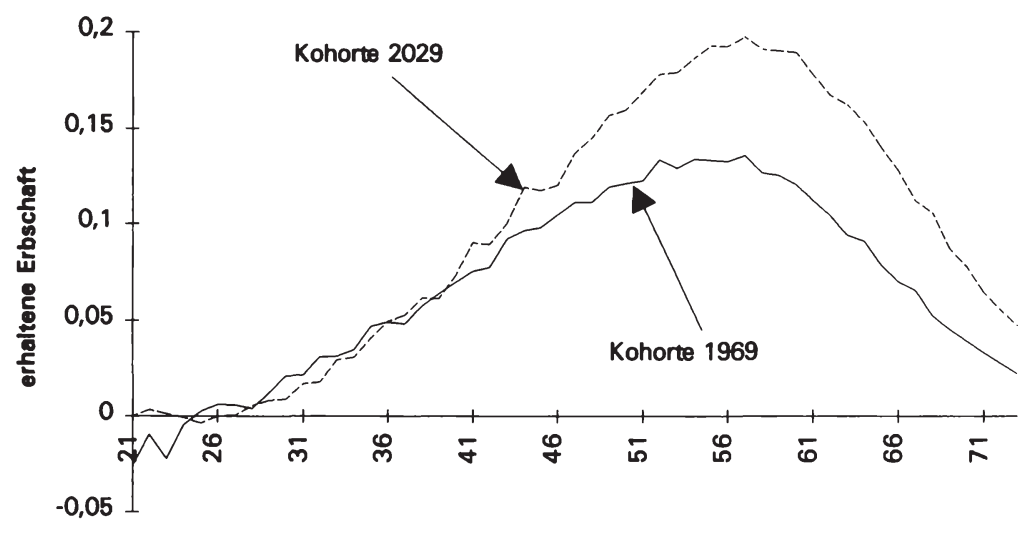

Aler

Quelle: Eigene Berechnungen

Die höchsten Erbschaften erhalten die Haushalte zwischen dem 50. und 60. Lebensjahr, doch auch in den Jahren davor und danach sind die Erbschaften von Bedeutung. Diese breite Streuung der Erbschaften ist ein wesentlicher Fortschritt meines Modells, denn der demographische Wandel schlägt sich auch in veränderten intergenerationalen Transfers nieder: Erstens verlagert die zunehmende Lebenserwartung die Spitze der Erbschaftsverteilung zu höheren Altern, wie Abbildung 4.3 zeigt. Kohorte 1969 erhält die höchsten Erbschaften mit 54 Jahren, Kohorte 2029 erreicht das Maximum mit 57 Jahren. Zweitens hat das Zahlenverhältnis zwischen den Generationen einen Einfluß. Wenn einer kleinen Kindergeneration eine große Elterngeneration gegenübersteht, dann erhält die Kindergeneration vergleichsweise hohe Erbschaften. Auch dieser Zusammenhang wird in Abbildung 4.3 deutlich. Die Kohorte 2029 erhält insgesamt höhere Erbschaften als die Kohorte 1969, denn das Zahlenverhältnis zwischen den Eltern der Kohorte 1969, also der Kohorte 1947, ist ungefähr eins, während die Kohorte 2002 als Elterngeneration der Kohorte 2029 deutlich stärker ist als die Kindergeneration.

Die teilweise negativen Erbschaften bis zum 25. Lebensjahr sind ein Artefakt. Die empfangenen Erbschaften in Abbildung 4.3 ergeben sich als Restgröße aus der Budgetrestriktion und diese Restgröße enthält neben den empfangenen Erbschaften auch den Effekt der Zuwanderung auf das durchschnittliche Vermögen 
einer Kohorte. Wenn der Migrationssaldo hoch ist, dann steigt nach Abbildung 3.1 vor allem die Stärke der jungen Kohorten. Da die Einwanderer annahmegemäß kein Vermögen mitbringen, senken sie das durchschnittliche Vermögen der jungen Kohorten. Wie die negativen Werte in Abbildung 4.3 zeigen, kann dieser Migrationseffekt zwischen dem 21. und 30. Lebensjahr größer sein als der Erbschaftseffekt. Daraus folgt, daß die Verteilung in Abbildung 4.3 die tatsächlich erhaltenen Erbschaften leicht unterschätzt.

Die Anpassung des Beitragssatzes zur umlagefinanzierten Rentenversicherung bei einer konstanten Nettoersatzquote von $72 \%$ stellt Abbildung 4.4 dar.

Abbildung 4.4: Entwicklung des Beitragssatzes zur umlagefinanzierten Rentenversicherung

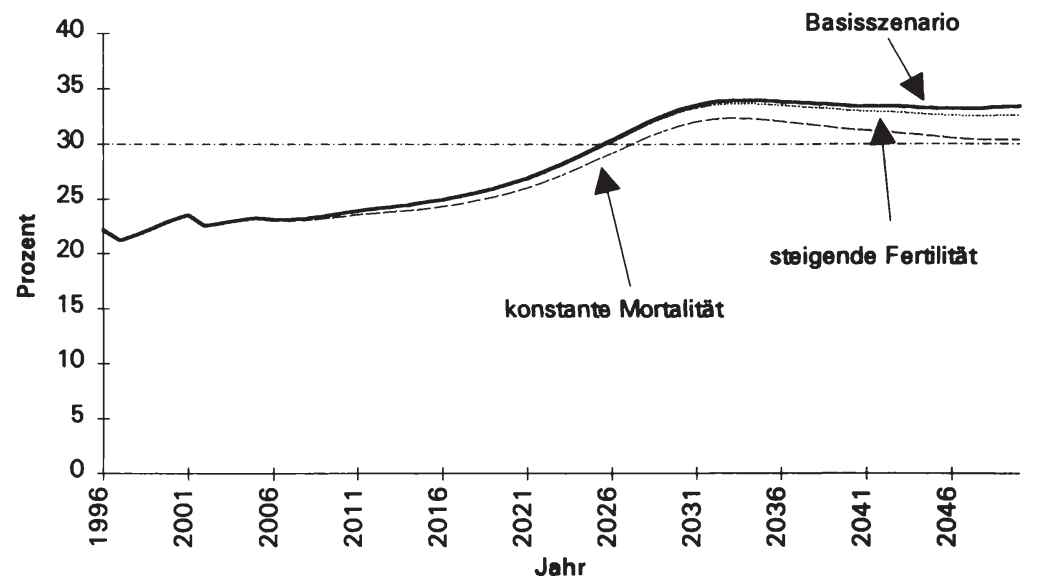

Quelle: Eigene Berechnungen

Der Ausgangswert von 22,2 \% im Jahr 1996 liegt über dem tatsächlichen Beitragssatz von 19,2\%. Diese Abweichung ist leicht zu erklären, denn die deutsche Rentenversicherung erhält erhebliche Zuwendungen aus dem Bundeshaushalt (etwa $20 \%$ der Rentenzahlungen, siehe Bundesministerium für Arbeit und Sozialordnung, 1996a). In meiner Simulation habe ich diesen Bundeszuschuß nicht berücksichtigt und damit bürde ich den Erwerbstätigen auch den Teil auf, den die Rentner durch ihre Steuerzahlungen zum Bundeszuschuß beitragen. Aus diesem 
Grund sind die simulierten Beitragssätze etwas höher als die tatsächlichen Beitragssätze.

Bis zum Jahr 2016 steigt der Beitragssatz relativ langsam an. Die Phase schnell steigender Beitragssätze beginnt nach dem Jahr 2020, wenn die Babyboom-Generation in Rente geht und dadurch den Beitragssatz im Basisszenario auf das Maximum von 33,9\% im Jahr 2034 erhöht. In den beiden anderen Szenarien wird der maximale Beitragssatz bereits im Jahr 2033 erreicht und beträgt 32,3\% („konstante Mortalität“) sowie 33,7\% (,steigende Fertilität“). Erneut wird deutlich, daß steigende Fertilität nur sehr langfristig zu einer Entspannung der Rentenfinanzen führt, während geringere Lebenserwartungen sehr viel schneller dämpfend auf die Beitragssätze einwirken.

Der maximale Beitragssatz im Basisszenario von 33,9\% deckt sich fast genau mit dem von Börsch-Supan (1995b) prognostizierten maximalen Beitragssatz von $34 \%$. Einen noch höheren Beitragssatz von $36 \%$ gibt Schmähl (1994a) für die Fortführung der umlagefinanzierten Rentenversicherung an, allerdings bezieht er sich auf die vor der Rentenreform 1992 gültigen Regelungen. Rodepeter (1995) berechnet für die nettolohnbezogenen Renten einen maximalen Beitragssatz von $35,6 \%$ im Jahr 2032. Im Zusammenhang mit den Politikszenarien im nächsten Abschnitt werde ich auf diese Diskussion zurückkommen.

Abbildung 4.5: Entwicklung der Erwerbsstundenquote

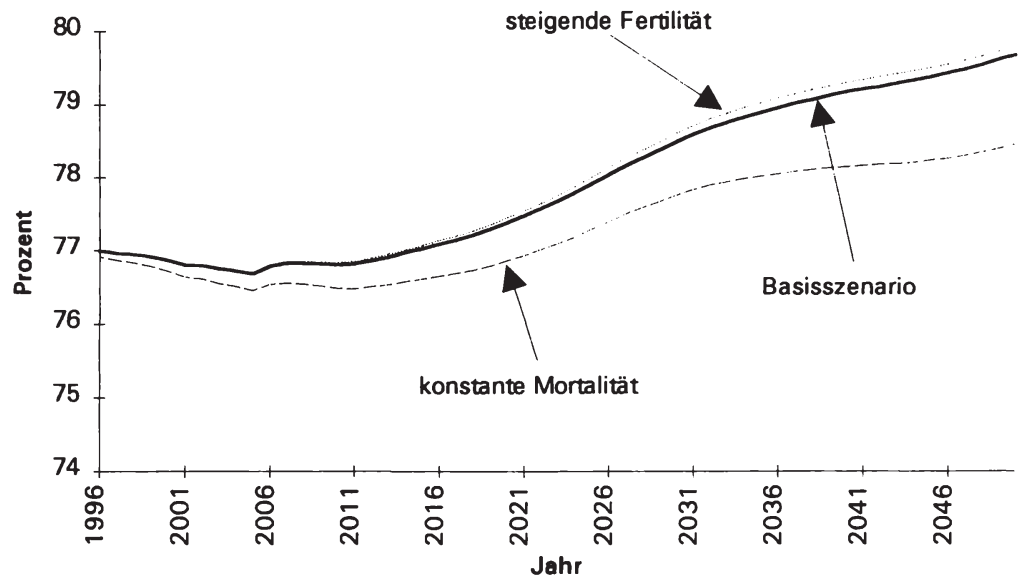

Quelle: Eigene Berechnungen 
Eine weitere interessante Kennzahl ist die Erwerbsstundenquote, definiert als die Anzahl der von den Arbeitnehmer geleisteten Arbeitsstunden geteilt durch die maximal mögliche Anzahl der Erwerbsstunden der Bevölkerung im erwerbsfähigen Alter. Das erwerbsfähige Alter erstreckt sich in meiner Simulation vom 21. Lebensjahr bis zum jeweils gültigen Verrentungsalter.

Bis zum Jahr 2005 nimmt die Erwerbsstundenquote leicht ab, um dann kontinuierlich anzusteigen. Zwischen den Jahren 2016 und 2030 ist der Anstieg stärker als in den übrigen Jahren. In diesen Jahren steigt der Beitragssatz zur Rentenversicherung deutlich und senkt die Nettolöhne. Drückt man die Entwicklung relativ zum Nettolohn im Jahr 1996 aus, so fällt der Nettolohn im Basisszenario von 97,8 \% im Jahr 2016 auf 87,7 \% im Jahr 2033. Trotzdem steigt das Arbeitsangebot. Offensichtlich überwiegt der Einkommenseffekt den Substitutionseffekt, denn der Gesamteffekt ist positiv. Im Szenario „konstante Mortalität“ ist die Reaktion der Erwerbsstundenquote schwächer, weil die Beitragssätze zur Rentenversicherung in diesem Szenario etwas langsamer ansteigen.

Die Entwicklung der Erwerbsstundenquote zeigt, daß die demographische Entwicklung im Basisszenario nicht zu einem Teufelskreis führt, vielmehr ist das Gegenteil der Fall: Die Erwerbstätigkeit steigt bei steigenden Beitragssätzen leicht an. In der hier unterstellten Modellwelt ist diese Reaktion sehr plausibel, denn die isoelastische Konsumnutzenfunktion mit $\gamma=3$ ist von höherer Ordnung als die logarithmische Freizeitnutzenfunktion, die im Grenzübergang für $\gamma$ gegen eins aus der isoelastischen Funktion entsteht. Weil ein höheres $\gamma$ zu einer höheren Glättung des entsprechenden Profils führt, sind die Haushalte in meiner Simulation stärker an einer Konsumglättung als an einer Freizeitglättung interessiert. Dieses Interesse wird in Abbildung 4.5 deutlich: Um ein relativ konstantes Konsumniveau bei steigenden Beitragssätzen verwirklichen zu können, reagieren die Haushalte auf die sinkenden Nettolöhne mit einem leicht zunehmenden Arbeitsangebot, um so das Einkommens- und Konsumniveau halten zu können. Prinzipiell ist diese Reaktion plausibel, fraglich ist jedoch, ob sie tatsächlich im "offiziellen“ Markt stattfindet. So stellt Rodepeter (1995) fest, daß steigende Beitragssätze zur Rentenversicherung den Trend zur Schwarzarbeit verstärken werden. Diese Tendenz kann mein Simulationsmodell nicht abbilden, denn hier gibt es nur offizielle Märkte - die Haushalte haben keine Möglichkeit, in die Schattenwirtschaft auszuweichen. Wenn sie diese Möglichkeit hätten, dann wäre der Druck auf die Beitragssätze noch stärker. Rodepeter zeigt aber auch, daß der Übergang zur nettolohnbezogenen Rente einen Teufelskreis prinzipiell ausschließt. Die Nettolohnanpassung verhindert Rückkopplungen der steigenden Beitragssätze auf das Arbeitsangebot, denn der Beitragssatz hängt dann nur vom Verhältnis der Rentner zu den Personen im erwerbsfähigen Alter ab und nicht von den tatsächlich Erwerbstätigen. 
Abbildung 4.6 zeigt die Veränderung des Pro-Kopf-Konsums bezüglich des Ausgangsjahres 1996 und schließt die Diskussion des Basisszenarios ab. Der ProKopf-Konsum gibt an, wie hoch der Konsum einer Person ist, wenn der gesamtwirtschaftliche Konsum genau gleich verteilt wird.

Abbildung 4.6: Entwicklung des Pro-Kopf-Konsums (Index $1996=1$ )

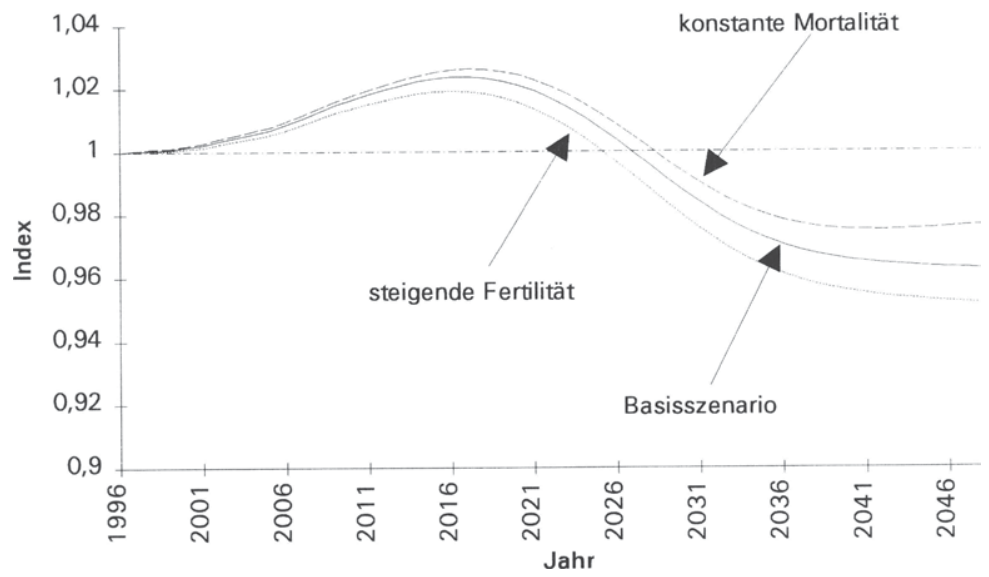

Quelle: Eigene Berechnungen

Der Pro-Kopf-Konsum verändert sich insgesamt relativ wenig, auch wenn die Wellenbewegung in Abbildung 4.6 wegen des Maßstabs sehr markant erscheint: Der Unterschied zwischen den Maxima von etwas mehr als 1,02 um das Jahr 2017 und dem Minimum von 0,95 im Szenario „steigende Fertilität“ ist nicht groß. So bewegt sich zum Beispiel die Kapitalintensität im gleichen Zeitraum (siehe Abbildung 4.1) erheblich mehr. Im Basisszenario steigt der Pro-Kopf-Konsum bis zum Jahr 2017, doch dann fällt er deutlich und liegt ab dem Jahr 2027 unter dem Wert des Ausgangsjahres. In den beiden anderen Szenarien ist die Entwicklung des Pro-Kopf-Konsums ähnlich, „steigende Fertilität“ fällt schon ab 2026 unter das Ausgangsniveau, „konstante Mortalität“ erst ab 2029. Anders als in den bisherigen Abbildungen unterscheidet sich das Szenario „steigende Fertilität" im Pro-Kopf-Konsum schon sehr bald von den anderen Szenarien, denn die höheren Geburtenraten erhöhen die Anzahl der Köpfe sofort. Dagegen reduziert im Szenario „konstante Mortalität" die geringere Lebenserwartung die Anzahl der Köpfe, so daß der Pro-Kopf-Konsum gegenüber dem Basisszenario steigt. 
Wichtig ist der Pro-Kopf-Konsum aber vor allem deshalb, weil er eine grobe Einschätzung der wirtschaftlichen Möglichkeiten eines durchschnittlichen Haushalts in verschiedenen Szenarien erlaubt. ${ }^{2}$ Der Output pro Arbeitnehmer ist für diese Einschätzung weniger geeignet, denn eine steigende Kapitalintensität erhöht zwar den Output pro Arbeitnehmer, durch die ebenfalls steigenden Ersatzinvestitionen steigt der Pro-Kopf-Konsum aber nicht in jedem Fall. ${ }^{3}$ In Abbildung 4.6 erreicht der Pro-Kopf-Konsum im Basisszenario im Jahr 2017 - mit dem Beginn der Verrentung der Babyboom-Generation - sein Maximum. In diesem Jahr könnten die Haushalte bei absolut gleichmäßiger Verteilung des Konsum am meisten konsumieren. Ein Blick auf die Kapitalintensität in Abbildung 4.1 macht deutlich, daß der Output pro Arbeitnehmer erst im Jahr 2029 sein Maximum erreicht und damit kein geeignetes $\mathrm{Ma} ß$ für die wirtschaftlichen Möglichkeiten eines durchschnittlichen Haushalts ist.

\section{Zusammenfassende Bewertung}

Das Basisszenario schreibt die im Jahr 1996 bestehenden Bedingungen im wesentlichen unverändert fort, nur das durchschnittliche Verrentungsalter steigt von 60 auf 61 Jahre. Bei diesen Annahmen steigt der Beitragssatz zur Rentenversicherung bis zum Jahr 2033 auf fast $34 \%$ des Bruttolohnes. Diese Entwicklung ist dramatisch, denn der Beitragssatz zur umlagefinanzierten Krankenversicherung ist in dieser Simulation nicht berücksichtigt. Da auch die Gesundheitsausgaben mit dem Alter stark ansteigen, ist ein Beitragssatz zur Krankenversicherung von über $15 \%$ im Jahr 2033 durchaus realistisch. Das Prognos-Gutachten (siehe Verband Deutscher Rentenversicherungsträger, 1995) geht für das Jahr 2040 von einem Beitragssatz von $16 \%$ aus und Börsch-Supan (1995a) prognostiziert für

2 Meine Simulation abstrahiert von technischem Fortschritt, der den Pro-Kopf-Konsum mit der Zeit erhöhen würde. Daher liegt der Schwerpunkt meiner Arbeit im Vergleich verschiedener Szenarien, denn dieser Vergleich wird durch technischen Fortschritt nicht wesentlich verändert.

3 Bei einer sehr hohen Kapitalintensität könnte Oversaving eintreten, d.h. die Sparquote würde die den Pro-Kopf-Konsum maximierende Golden Rule Saving Rate übersteigen. In diesem Fall wäre der Kapitalstock zu groß, es müßte ein zu großer Teil des Outputs zur Erhaltung des Kapitalstocks aufgebracht werden. Nach Barro und Sala-i-Martin (1995) gilt jedoch folgendes Ergebnis: Wenn die Haushalte in überlappenden Generationen Modellen (namentlich dem Ramsey Modell) einen unendlichen Zeithorizont haben, dann kann Oversaving nicht auftreten. In meinem Modell ist der Zeithorizont der Haushalte aber endlich, während die Volkswirtschaft einen unendlichen Zeithorizont hat. Die heute lebenden Haushalte können daher aus ihrer Sicht optimale Entscheidungen fällen, die aus der Sicht eines sozialen Planer mit unendlichem Zeithorizont nicht optimal sind. Für Einzelheiten verweise ich auf Barro und Sala-i-Martin (1995), Kapitel 3. 
das Jahr 2030 Krankenversicherungsbeiträge von über $17 \%$. So ist denkbar, daß allein die Beitragssätze für die Renten- und Krankenversicherung das Bruttogehalt um mehr als $50 \%$ reduzieren. Dazu kommen dann noch die Pflegeversicherung und die allgemeinen Steuern zur Finanzierung des Staatshaushaltes sowie andere Abgaben. Folglich eröffnet das Basisszenario die Aussicht auf einen schnell zunehmenden Unterschied zwischen Brutto- und Nettolohn, der bei Belastungen von über $70 \%$ in den dreißiger Jahren des nächsten Jahrhunderts sein Maximum erreicht. $\mathrm{Da}$ sich in diesem Fall enorme Probleme für den Generationenvertrag - auf dem die umlagefinanzierte Sozialversicherung beruht - ergeben, ist offensichtlich. Denn wenn der Generationenvertrag aufrecht erhalten würde, wäre ein weiter zunehmender Druck in Richtung Schattenwirtschaft mit allen seinen negativen Konsequenzen sehr wahrscheinlich. Im folgenden Abschnitt untersuche ich daher die vier grundsätzlichen Möglichkeiten, mit denen die Politik der demographischen Entwicklung begegnen kann.

Der Vergleich des Basisszenarios mit den politikfernen Szenarien „konstante Mortalität" und „steigende Fertilität“ zeigt, daß diese beiden Parameter zeitlich sehr unterschiedlich auf die makroökonomischen Variablen einwirken: Steigende Geburtenraten wirken sich sehr langfristig aus, während konstante Mortalitätsraten sehr viel schneller zu merklichen Veränderungen führen. Ich habe die beiden politikfernen Szenarien zusammen mit dem Basisszenario dargestellt, um die Sensitivität des Basisszenarios hinsichtlich der Basisvariante meiner Bevölkerungsprognose aufzuzeigen. So wird deutlich, da $\beta$ auch die politikfernen Parameter Fertilität und Mortalität Auswirkungen auf die makroökonomischen Variablen haben und Spielraum für verschiedene Szenarien bieten. Im nächsten Abschnitt unterlege ich meinen Politikszenarien jedoch einheitlich die Basisversion meiner Bevölkerungsprognose, denn dann geht es mir um den Vergleich verschiedener Reformen des Rentensystems.

\subsection{Vier Politikszenarien}

Die Politik kann - einzeln oder kombiniert - mit vier unterschiedlichen Maßnahmen auf den demographischen Wandel reagieren. In der Einführung zu diesem Kapitel habe ich sie mit der Wiedergabe von Breyers (1996) Abgrenzung schon erwähnt - hier stelle ich sie noch einmal schlagwortartig zusammen:

- Höheres Verrentungsalter

- Höhere Einwanderungszahlen

- Umstieg auf eine kapitalgedeckte Altersversorgung 
- Sinkende Rentenersatzquote

Die vier Politikszenarien sollen zeigen, ob und wie die gesamtwirtschaftliche Entwicklung gegenüber dem Basisszenario verbessert werden kann. Jedes Politikszenario verändert jeweils eine der vier Rahmenbedingungen, so da $\beta$ man aus der Einzelbetrachtung auf die Wirkung von Politiken schließen kann, die mehrere Einzelmaßnahmen kombinieren.

Das erste Politikszenario heißt „Rentenalter erhöhen“. Das Verrentungsalter wird stärker als im Basisszenario angehoben: Im Jahr 1996 von 61 auf 62 Jahre, also geht die 1933 geborene Kohorte noch mit Vollendung ihres 61. Lebensjahres (im Jahr 1994) in Rente, die 1934 geborene Kohorte arbeitet dagegen bis zur Vollendung ihres 62. Lebensjahres (im Jahr 1996). Im Jahr 2001 steigt das Verrentungsalter um ein weiteres Jahr auf 63 und erreicht im Jahr 2007 mit 64 schließlich das bis zum Ende des Simulationszeitraums geltende Niveau. Das Szenario „Rentenalter erhöhen“ ist den von der Bundesregierung auf den Weg gebrachten Maßnahmen ähnlich. Das im Juli 1996 verabschiedete Programm für mehr Wachstum und Beschäftigung (siehe Bundesministerium für Arbeit und Sozialordnung, 1996b) sieht vor: Schrittweise Anhebung der Altersgrenze für Frauen von heute 60 Jahren auf 65 Jahre bis zum Ende des Jahres 2004. Ebenso wird die Altersgrenze langjährig Versicherter von heute 63 Jahren auf 65 Jahre angehoben. Beide Maßnahmen sollen dem Trend zur Frühverrentung entgegenwirken und so das durchschnittliche Verrentungsalter merklich erhöhen.

Das zweite Szenario untersucht, welche Auswirkungen eine gegenüber dem Basisszenario verdoppelte Einwanderung hat - daher der Name „Einwanderung verdoppeln“. In diesem Szenario steigt der Migrationssaldo bis zum Jahr 2000 an und bleibt dann mit 400.000 Einwanderern pro Jahr bis zum Ende des Simulationszeitraums konstant. Die Altersverteilung der zusätzlichen Einwanderer gleicht in allen Jahren der in Abbildung 3.1 gezeigten Verteilung, also stärken die vergleichsweise jungen Einwanderer die Erwerbsbevölkerung ganz wesentlich. Alle anderen Rahmenbedingungen entsprechen denen des Basisszenarios.

Einen völlig anderen Ansatz verfolgt das dritte Politikszenario. Es trägt den Namen „Kapitaldeckung einführen“, weil es den nach 1964 geborenen Kohorten keine Renten aus der umlagefinanzierten Sozialversicherung zuspricht. Die 1965 und später geborenen Kohorten sind 1996 noch am Anfang ihres Berufslebens und haben daher über dreißig Jahre Zeit, eigene Ersparnisse für den Ruhestand zu bilden. Ob diese Ersparnisse in Immobilien, Assets, Lebensversicherungen oder im Rahmen einer vom Staat organisierten kapitalgedeckten Rentenversicherung angelegt werden, ist unter den Annahmen meines Simulationsmodells nicht von Bedeutung, solange sie bei vorzeitigem Tod vererbt werden können. Wesentlich ist, daß ein Kapitalstock aufgebaut wird. Der Umstieg von der Umlagefinanzie- 
rung auf die Kapitaldeckung passiert ohne abfedernde Maßnahmen. Die 1965 geborene Kohorte erhält also keine Renten aus der auslaufenden umlagefinanzierten Rentenversicherung, muß aber während ihres ganzen Erwerbslebens Beitragssätze entrichten, um die Renten der vor 1965 geborenen Kohorten zu finanzieren. Dieser drastische Umstieg bedeutet, daß es im Szenario „Kapitaldeckung einführen" Kohorten gibt, die eine doppelte Last tragen müssen: die Beiträge für die noch bestehende umlagefinanzierte Rentenversicherung und die Ersparnisse für die eigene Altersversorgung.

Schließlich reagiert das vierte Szenario „Ersatzquote senken“ mit einer schrittweise sinkenden Netto-Ersatzquote auf den demographischen Wandel. Nach dem Jahr 1997 wird die Netto-Ersatzquote jährlich um einen Prozentpunkt gesenkt, bis sie im Jahr 203930 \% erreicht. Durch diesen allmählichen Übergang wird einem Urteil des Bundesverfassungsgerichts Rechnung getragen, nach dem die im bestehenden Rentensystem erworbene Ansprüche zum Teil als Eigentum zu betrachten sind. Das Szenario „Kapitaldeckung einführen“ hätte mit dem Urteil des Bundesverfassungsgerichts Schwierigkeiten und ist daher eher zur Illustration der grundsätzlichen Auswirkungen eines Umstiegs auf Kapitaldeckung zu betrachten. Dagegen werden Szenarien wie „Ersatzquote senken“ ernsthaft diskutiert. Das Szenario „Ersatzquote senken“ kann als Grundrente interpretiert werden: Wenn die Ersatzquote auf $30 \%$ fällt, dann bekommen die Rentner einer Kohorte im Mittel $30 \%$ des durchschnittlichen Netto-Lebensarbeitseinkommens ihrer Kohorte als jährliche Rente. Wie dieses $30 \%$-Mittel auf die einzelnen Haushalte der Kohorte aufgeteilt wird, ist nicht Gegenstand des Simulationsmodells, aber es ist denkbar, daß alle Haushalte genau das $30 \%$-Mittel als Grundrente bekommen. Für einkommensschwache Haushalte wäre das relativ viel, für einkommensstarke Haushalte wäre das relativ wenig. Daher werden besonders die einkommensstarken Haushalte eigene Ersparnisse zur Sicherung ihres Konsums im Alter bilden.

Die Arbeit von Buslei und Kraus (1996) simuliert einen allmählichen Umstieg auf ein partiell kapitalgedecktes Verfahren. In der politischen Diskussion in Deutschland werden Grundrentensysteme vor allem von Kurt Biedenkopf und Meinhard Miegel propagiert, siehe Miegel (1985). Tatsächlich existieren in einigen europäischen Ländern Grundrentensysteme, teilweise in Mischform mit lohnabhängigen Zusatzrenten. Beispiele sind Dänemark, Finnland, Großbritannien, Irland, die Niederlande und Schweden (siehe Bundesministerium für Arbeit und Sozialordnung, 1996a).

Tabelle 4.1 stellt das Basisszenario und die vier Politikszenarien übersichtlich zusammen. Die Rahmenbedingungen, die in den Politikszenarien gegenüber dem Basisszenario abweichen, sind schraffiert hervorgehoben. 
Tabelle 4.1: $\quad$ Das Basisszenario und die vier Politikszenarien

\begin{tabular}{|c||c|c|c|}
\hline Szenario & Rentenalter & Migrationssaldo & Ersatzquote \\
\hline \hline Basisszenario & 61 & 200.000 & $72 \%$ \\
\hline $\begin{array}{c}\text { Rentenalter } \\
\text { erhöhen }\end{array}$ & $\begin{array}{c}\text { bis 1994: 61 } \\
\text { ab 1995: 62 } \\
\text { ab 2001: 63 } \\
\text { ab 2007: } 64\end{array}$ & 200.000 & $72 \%$ \\
\hline $\begin{array}{c}\text { Einwanderung } \\
\text { verdoppeln }\end{array}$ & 61 & 400.000 & $72 \%$ \\
\hline $\begin{array}{c}\text { Kapitaldeckung } \\
\text { einführen }\end{array}$ & 61 & 200.000 & $\begin{array}{c}\text { bis 2026: } 72 \% \\
\text { ab 2027: } 0 \%\end{array}$ \\
\hline $\begin{array}{c}\text { Ersatzquote } \\
\text { senken }\end{array}$ & 61 & 200.000 & $\begin{array}{c}\text { bis 1997: } 72 \% \\
\text { dann sinkend } \\
\text { auf 30\% in } \\
2039\end{array}$ \\
\hline
\end{tabular}

Quelle: Eigene Darstellung

Das Basisszenario und die Szenarien „Rentenalter erhöhen“ sowie „Einwanderung verdoppeln“ bezeichne ich als systemerhaltende Szenarien, weil sie die Grundstrukturen der heutigen umlagefinanzierten Rentenversicherung kaum verändern. Dagegen bedeuten die Szenarien „Kapitaldeckung einführen“ und "Ersatzquote senken" deutliche Reformen des heutigen Systems, also nenne ich sie systemverändernde Szenarien.

Die folgenden Abbildungen zeigen, wie sich die wichtigsten endogenen Variablen in den jeweiligen Szenarien vom Jahr 1996 bis zum Jahr 2050 entwickeln. Jede Abbildung vergleicht eine endogene Variable in den vier Politikszenarien mit dem Basisszenario, das durch eine dickere Linie gekennzeichnet ist.

Abbildung 4.7 zeigt die Kapitalintensität relativ zum Indexjahr 1996, das in den vier Politikszenarien und dem Basisszenario weitgehend vergleichbar ist, denn die Politikszenarien ändern erst ab dem Jahr 1996 die Rahmenbedingungen.

Die beiden systemverändernden Szenarien „Kapitaldeckung einführen“ und „Ersatzquote senken“ unterscheiden sich deutlich vom Basisszenario, im Gegensatz zu den beiden systemerhaltenden Szenarien "Rentenalter erhöhen" und „Einwanderung verdoppeln“. Bei einem Umstieg auf eine kapitalgedeckte Altersversorgung steigt die Kapitalintensität bis zum Jahr 2050 um mehr als das Dop- 
pelte ihres Ausgangswerts im Jahr 1996 - verursacht durch den stark steigenden Kapitalstock der Gesamtwirtschaft. Die privaten Haushalte sparen im Vergleich zur umlagefinanzierten Rentenversicherung einen größeren Teil ihres Einkommens für ihren Konsum im Rentenalter. Weil die Kapitalintensität positiv mit dem Lohn und Output korreliert ist, kann der Konsum trotzdem höher sein als im Basisszenario, denn der höhere Output vergrößert den „Kuchen“, der zwischen Konsum und Investitionen zu verteilen ist. Ob die höhere Kapitalintensität im Szenario „Kapitaldeckung einführen“ tatsächlich zu einem höheren Pro-KopfKonsum führt, wird Abbildung 4.12 später offenbaren.

Abbildung 4.7: Entwicklung der Kapitalintensität in den Politikszenarien (Index $1996=1)$

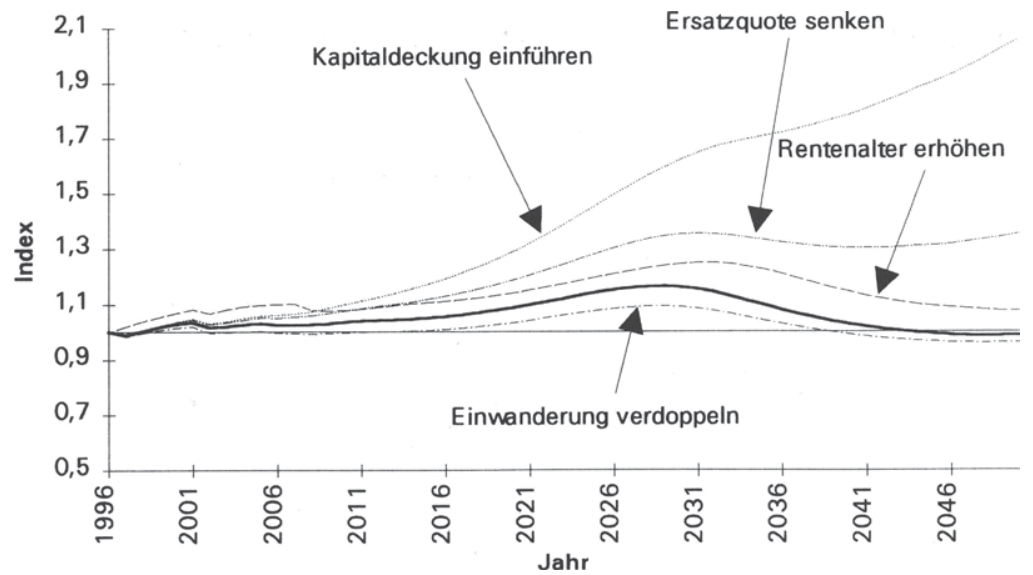

Quelle: Eigene Berechnungen

Weit weniger stark als im Szenario „Kapitaldeckung einführen“ steigt die Kapitalintensität im Szenario „Ersatzquote senken“, das auch als Grundrente interpretiert werden kann. Auch hier steigt die private Ersparnis, denn die Haushalte, denen die Grundrente zu wenig ist, müssen durch eigene Altersvorsorge für ihren Konsum nach der Verrentung sorgen. Dadurch beträgt die Kapitalintensität im Jahr 2050 das 1,35-fache ihres Ausgangswerts von 1996.

Die systemerhaltenden Szenarien „Rentenalter erhöhen“ und „Einwanderung verdoppeln" unterscheiden sich nur wenig vom Basisszenario. Bei einer schrittwei- 
sen Erhöhung des Rentenalters ist die Kapitalintensităt ab Mitte der zwanziger Jahre des nächsten Jahrhunderts etwas höher als im Basisszenario. Das Szenario „Einwanderung verdoppeln“ führt sogar zu einer geringfügig niedrigeren Kapitalintensität als das Basisszenario. Dieser Effekt geht auf die höhere Anzahl der Arbeitnehmer zurück, denn offensichtlich ist der positive Effekt der Einwanderer auf die Altersverteilung zu gering, um zu einer nachhaltigen Verbesserung des gesamtwirtschaftlichen Kapitalangebots zu führen.

Alle Szenarien - auch „Kapitaldeckung einführen“ - zeigen einen deutlichen $\mathrm{Zu}$ sammenhang zwischen Demographie und Kapitalintensität: Nach der Verrentung der Babyboom-Generation bis zum Jahr 2030 geht die Steigung im Szenario „Kapitaldeckung einführen“ leicht zurück, im Szenario „Ersatzquote senken“ wird sie nur vorübergehend negativ, und in den anderen Szenarien bleibt sie negativ.

Abbildung 4.8: Entwicklung der Sparquote in den Politikszenarien

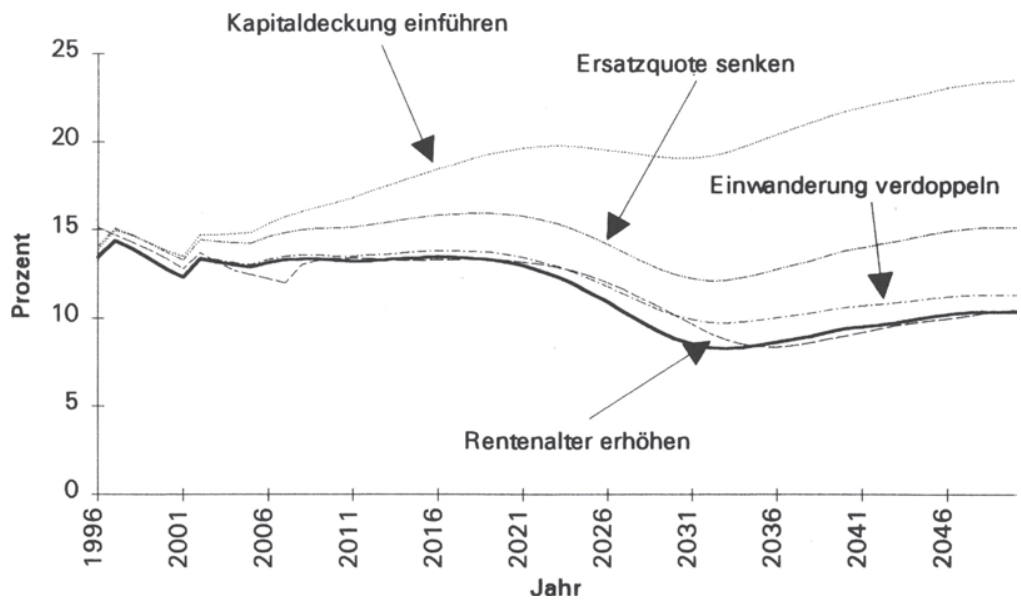

Quelle: Eigene Berechnungen

Die Entwicklung der Sparquote steht natürlich in einem engen Zusammenhang mit der Entwicklung der Kapitalintensität, denn die private Ersparnis bestimmt das gesamtwirtschaftliche Kapitalangebot. Auch in Abbildung 4.8 unterscheiden sich die systemverändernden Szenarien „Kapitaldeckung einführen“ und „Ersatzquote senken“ deutlich vom Basisszenario und den systemerhaltenden 
Szenarien "Rentenalter erhöhen" und „Einwanderung verdoppeln". Die private Ersparnis ist im Jahr 2050 mit 23,4\% im Szenario „Kapitaldeckung einführen“ um fast zehn Prozentpunkte höher als im Minimum von 13,5\% im Jahr 2001. Diese Entwicklung verdeutlicht den zentralen Unterschied von kapitalgedeckten und umlagefinanzierten Rentensystemen. Kapitaldeckung bedeutet, daß der Konsum im Alter durch einen während des Erwerbslebens aufgebauten Kapitalstock finanziert wird. Dieses Ansparen im Szenario „Kapitaldeckung einführen“ ist nicht nur die Ursache für die steigende Sparquote in Abbildung 4.8, sondern auch für die steigende Kapitalintensität in Abbildung 4.7. Das Szenario „Ersatzquote senken" mischt Elemente des Umlageverfahrens mit Elementen der Kapitaldeckung und hat daher eine deutlich höhere Sparquote als die Szenarien mit reinem Umlageverfahren. Es übertrifft die Sparquote des Ausgangsjahres von $14,5 \%$ mit der Sparquote von $15,1 \%$ im Jahr 2050 bei einem zwischenzeitlichen Rückgang auf 12,1 \% im Jahre 2033. Alle anderen Szenarien haben im Jahr 2050 geringere Sparquoten als im Ausgangsjahr 1996, denn bei einer umlagefinanzierten Rentenversicherung wird kein Kapitalstock aufgebaut, vielmehr werden die in einem Jahr eingezahlten Beiträge im gleichen Jahr als Renten ausbezahlt.

In allen Szenarien fällt das Ausscheiden der Babyboom-Generation aus dem Erwerbsleben mit den Minima der Sparquoten um das Jahr 2033 zusammen. Der in meiner Simulation angenommene einheitliche Lebenszyklus der Haushalte führt dazu, daß die Haushalte nach ihrem 47. Lebensjahr, wenn die Kinder einen eigenen Haushalt gegründet haben, in die „Hauptsparphase“ eintreten. Bis zur Verrentung bauen sie ihr Vermögen kräftig aus. Im Ruhestand wird das Vermögen anfänglich wieder reduziert, um das erreichte Konsumniveau zu halten. Erst mit höherem Alter geht der Konsum zurück und das Vermögen wird kaum noch vermindert (siehe Abbildungen 2.11 und 2.12). Das erklärt den deutlichen Rückgang der Sparquote nach der Verrentung der Babyboom-Generation.

Die unterschiedliche Entwicklung der Sparquote in den Szenarien hat natürlich Auswirkungen auf die Erbschaften, die wegen der unsicheren Lebenserwartung in meiner Simulation über den ganzen Zeitraum verteilt werden, in dem noch Eltern der Kindergeneration leben (siehe Abbildung 4.3). Vergleicht man wieder die Kohorte 1969 mit der Kohorte 2029, so erkennt man große Unterschiede in den erhaltenen Erbschaften. Im Basisszenario beträgt das Verhältnis zwischen erhaltenen Erbschaften und Netto-Lebensarbeitseinkommen 4,3\% für die Kohorte 1969 und 6,6 \% für die Kohorte 2029. Im Szenario „Ersatzquote senken“ hat die Kohorte 1969 mit 4,6\% ein ähnliches Verhältnis zwischen erhaltenen Erbschaften und Lebensarbeitseinkommen wie im Basisszenario, für die Kohorte 2029 ist das Verhältnis mit 8,1\% aber deutlich höher. Noch höher ist das entsprechende Verhältnis für die Kohorte 2029 mit 9,6\% im Szenario „Kapitaldeckung einführen". An diesen Zahlen wird deutlich, wie die unsichere Lebenserwartung das Simulationsmodell gegenüber Modellen mit sicherer Lebenserwartung entschei- 
dend weiterentwickelt. Bei sicherer Lebenserwartung hat die höhere Vermögensakkumulation bei teilweise oder vollständig kapitalgedeckten Alterssicherungen keine Auswirkung auf die Erbschaften, denn bis zum sicheren Todeszeitpunkt ist der Alterssicherungsteil des Vermögens gerade aufgezehrt. Bei unsicherer Lebenserwartung wird ein Teil des Alterssicherungsvermögens durch Accidental Bequests an die nächsten Generationen weitergegeben. Diese Verkettung von Erbschaften und Alterssicherungsvermögen ist ein zentraler Fortschritt meines Simulationsmodells, denn zweifellos sind Vermögensübertragungen ein wichtiger Aspekt des demographischen Wandels, auf den beispielsweise die Deutsche Bundesbank ausdrücklich hinweist (Deutsche Bundesbank, 1993).

Abbildung 4.9: Entwicklung des Beitragssatzes zur umlagefinanzierten Rentenversicherung in den Politikszenarien

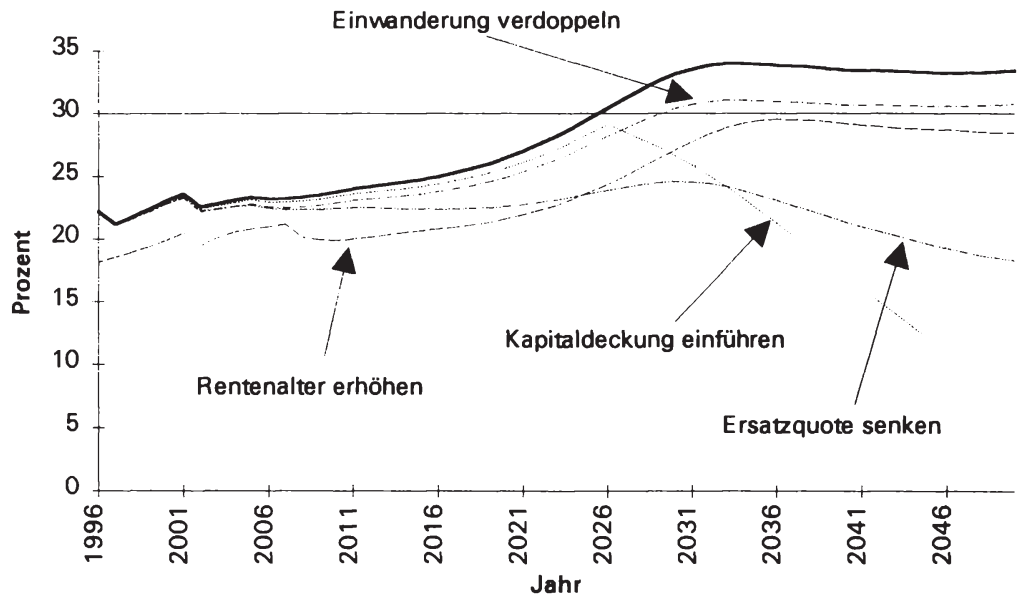

Quelle: Eigene Berechnungen

Das Basisszenario ist durch die höchsten Beitragssätze gekennzeichnet: Das Maximum beträgt 33,9\% im Jahr 2033. Etwas günstiger entwickeln sich die Beitragssätze im Szenario „Einwanderung verdoppeln“; mit 31,0\% liegt das Maximum um 2,9 Prozentpunkte niedriger als im Basisszenario. Das Szenario "Rentenalter erhöhen" nimmt einen ähnlichen Verlauf wie das Basisszenario und das Szenario „Einwanderung verdoppeln“, aber der maximale Beitragssatz, der im Jahr 2036 gilt, ist mit 29,6\% erheblich geringer als im Basisszenario. Bei der Umstellung auf eine kapitalgedeckte Altersversorgung entwickelt sich der Bei- 
tragssatz bis zum Jahr 2027 weitgehend parallel zum Basisszenario, denn erst ab dem Jahr 2027 erhalten die dann in Rente gehenden Kohorten keine Leistungen mehr aus der umlagefinanzierten Rentenversicherung. Mit dem Auslaufen der umlagefinanzierten Rentenversicherung gehen die Beitragssätze dann schnell zurück.

Vergleicht man die Beitragssätze in den systemerhaltenden Szenarien mit anderen Prognosen, zeigen sich Gemeinsamkeiten. Das Gutachten des Prognos-Instituts, siehe Verband Deutscher Rentenversicherungsträger (1995), prognostiziert für die obere Variante (hohe Migration) einen Beitragssatz von 26,3 \% im Jahr 2030 und für die untere Variante (niedrige Migration) von 28,5\%. Mit 26,9\% im Jahr 2030 veröffentlicht Schmähl (1994a) eine sehr ähnliche Zahl. Da meine Simulation keinen Bundeszuschuß zur Rentenversicherung enthält, ist der maximale Beitragssatz im Szenario „Rentenalter erhöhen“ mit 29,6 \% etwas höher. Hinzu kommen die vereinfachenden Annahmen bezüglich der Einwanderer, die in meiner Simulation die gleichen Fertilitäts- und Mortalitätsraten wie die einheimische Bevölkerung haben. Tatsächlich ist die Lebenserwartung der Einwanderer geringer und die Geburtenrate höher - beides würde die Beitragssätze weiter entlasten. So wird auch verständlich, warum die Zunahme der Migration von 200.000 auf 400.000 jährliche Einwanderer ab dem Jahr 2000 im Szenario „Einwanderung verdoppeln" mit 2,9 Prozentpunkten relativ wenig zur Dämpfung des maximalen Beitragssatzes beiträgt. Börsch-Supan (1995a) schätzt den Effekt eines von 100.000 auf 300.000 erhöhten Migrationssaldos höher ein: Der maximale Beitragssatz fällt um etwa vier Prozentpunkte.

Die im Szenario „Rentenalter erhöhen“ gegenüber dem Basisszenario eintretende Minderung des maximalen Beitragssatzes beträgt 4,3 Prozentpunkte. Schmähl (1994b) berechnet für das Jahr 2030 eine Beitragsentlastung von 1,5 bis 2,5 Prozentpunkten, wenn das durchschnittliche Rentenalter um ein Jahr erhöht wird. Das Szenario „Rentenalter erhöhen“ setzt das durchschnittliche Rentenalter über einen Zeitraum von zwölf Jahren insgesamt um drei Jahre hoch und paßt gut zu dem von Schmähl angegebenen Rahmen. Etwas geringer schätzt Börsch-Supan (1995a) die Entlastung des Beitragssatzes ein: Wenn das durchschnittliche Rentenalter von 60 auf 62,5 Jahre angehoben wird, dann reduziert sich der maximale Beitragssatz in seiner Simulation um etwas mehr als zwei Prozentpunkte.

Mittelfristig hat das Szenario „Ersatzquote senken“ den nachhaltigsten Effekt auf die Beitragssätze, die bis zum Jahr 2030 zwar auf 24,6\% ansteigen, dann aber bis auf 18,3\% im Jahr 2050 zurückgehen. Erreicht werden diese im Vergleich zu den systemerhaltenden Szenarien günstigen Beitragssätze durch die zurückgehenden Netto-Ersatzquoten. Von $72 \%$ im Jahr 1997 fallen die Ersatzquoten jährlich um einen Prozentpunkt und erreichen im Jahr 2039 das Ziel von 30 \%. Das Simulationsmodell von Buslei und Kraus (1996) untersucht ebenfalls einen graduellen 
Übergang auf ein niedrigeres Rentenniveau. Ihr anvisiertes Rentenniveau ist mit $45 \%$ wesentlich höher als das Rentenniveau im Szenario „Ersatzquote senken“. Trotzdem steigen die Beitragssätze in der Simulation von Buslei und Kraus nicht über $22 \%$. Dieses Ergebnis halte ich für wenig realistisch, denn Buslei und Kraus unterstellen eine feste Lebenszeit von 76 Jahren und berücksichtigen nicht die steigende Lebenserwartung.

Wenn man nur die Beitragssätze zum Vergleich der Szenarien heranzieht, berücksichtigt man nicht den Sparbeitrag, den die Haushalte in kapitalgedeckten Systemen aufbringen müssen. Abbildung 4.10 gibt die Summe der Sparquote und der Beitragssätze für die betrachteten Szenarien wieder. Diese Addition ist nicht unproblematisch, denn der Beitragssatz zur Rentenversicherung hat bei versicherungsmathematisch unfairen Systemen auch den Charakter einer Steuer, während die Sparquote das eigene Vermögen betrifft.

Abbildung 4.10: Summe aus Sparquote und Beitragssatz in den Politikszenarien

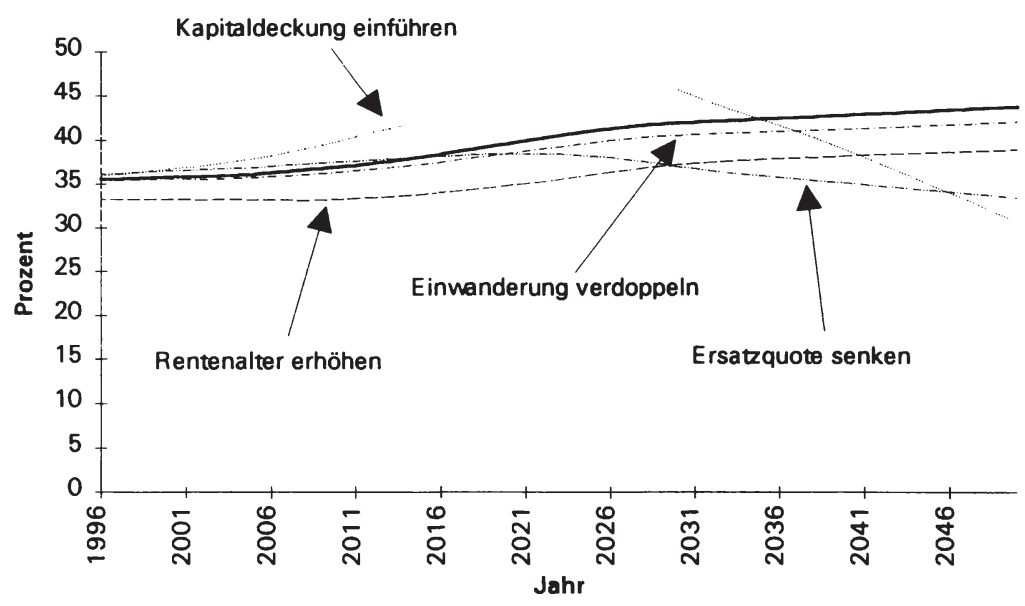

Quelle: Eigene Berechnungen

Die Summe aus Sparquote und Beitragssatz zur Rentenversicherung ist ein Proxi für die Gesamtbelastung. „Kapitaldeckung einführen“ bedeutet mittelfristig die höchste Belastung, denn die Übergangsgeneration muß Beiträge zur umlagefinanzierten Rentenversicherung zahlen - von der sie nicht profitiert - und für ihre eigene kapitalgedeckte Altersversorgung sparen. Diese doppelte Belastung ver- 
schwindet erst, wenn die umlagefinanzierte Rentenversicherung nach dem Jahr 2027 keine neuen Rentner mehr finanzieren muß. Dann sinkt die Gesamtbelastung im Szenario „Kapitaldeckung einführen“ schnell und ist ab dem Jahr 2046 am geringsten. Kurz- bis mittelfristig am günstigsten ist die Gesamtbelastung im Szenario „Rentenalter erhöhen“, denn in diesem Szenario kommen zwei Effekte zusammen: Erstens sind die Beitragssätze wegen der höheren Anzahl der Beitragszahler und der zurückgehenden Anzahl der Beitragsempfänger niedriger und zweitens verlängert sich die Lebensphase mit der höchsten Sparneigung, die sich in meiner Simulation vom 47. Lebensjahr bis zur Verrentung erstreckt. Aus Abbildung 4.10 wird deutlich, daß beide Effekt nur das Niveau der Gesamtbelastung verändern, denn die Profile in den systemerhaltenden Szenarien verlaufen weitgehend parallel. Das Szenario „Ersatzquote senken“ verläuft bis zum Jahr 2016 ähnlich wie das Basisszenario, aber dann sinkt die Gesamtbelastung. Zwischen den Jahren 2030 und 2045 ist die Gesamtbelastung im Vergleich zu den anderen Szenarien am geringsten. Nach dem Jahr 2046 ist der Umstieg auf eine vollständig kapitalgedeckte Alterssicherung günstiger. Im Gegensatz zu der steigenden Gesamtbelastung in den systemerhaltenden Szenarien nimmt die Gesamtbelastung im Szenario „Ersatzquote senken“ aber weiter ab.

Abbildung 4.11: Entwicklung der Erwerbsstundenquote in den Politikszenarien

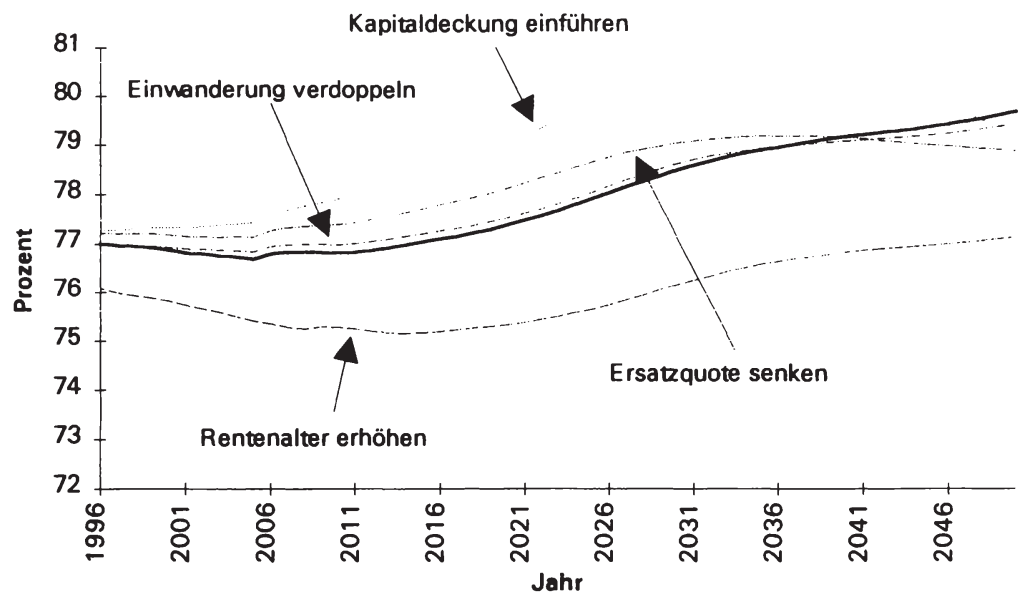

Quelle: Eigene Berechnungen 
Abbildung 4.11 vergleicht in den jeweiligen Szenarien die Entwicklung der Erwerbsstundenquote - definiert als der Prozentsatz der geleisteten Arbeitsstunden an den maximal möglichen Arbeitsstunden der Erwerbsbevölkerung. Aus den im Szenario „Kapitaldeckung einführen“" mittelfristig höchsten Erwerbsstundenquoten wird erneut die doppelte Belastung der Übergangsgeneration deutlich. Es gilt die gleiche Erklärung wie bei den Erwerbsstundenquoten in Abbildung 4.5: Konsumglättung ist den Haushalten wichtiger als Freizeitglättung, also bewegt die doppelte Belastung die Haushalte der Übergangsgeneration zu einem höheren Arbeitsangebot. Wenn die Belastung nach dem Jahr 2030 sinkt, dann geht auch die Erwerbsstundenquote zurück. Die vergleichsweise niedrigen Erwerbsstundenquoten im Szenario „Rentenalter erhöhen“ erklären sich ebenfalls durch die Präferenzen, denn die Haushalte nehmen ihr Arbeitsangebot zurück, wenn sie der Verrentung näher kommen. Daher sind die Erwerbsstundenquoten in Altersklassen älterer Arbeitnehmer, die bei einem höheren Verrentungsalter an Gewicht gewinnen, geringer.

Der Anstieg der Erwerbsstundenquoten in den systemerhaltenden Szenarien kann mit den Ergebnissen des Prognos-Rentengutachtens (siehe Verband Deutscher Rentenversicherungsträger, 1995) verglichen werden. Beide Varianten des Prognos-Gutachtens gehen von einem Anstieg der Erwerbsquoten um zehn Prozentpunkte zwischen 1992 und 2030 aus. Dieser Anstieg fällt stärker aus als in Abbildung 4.11. Allerdings wird die Erwerbsbeteiligung im Prognos-Gutachten nicht endogen bestimmt sondern exogen vorgegeben. Ein wichtiges Argument für die stärker steigenden Erwerbsquoten des Prognos-Gutachtens ist die steigende Erwerbsbeteiligung von Frauen - diesen Effekt berücksichtigt meine Simulation nicht.

Der Vergleich des Pro-Kopf-Konsums in den Politikszenarien in Abbildung 4.12 ergibt ähnliche Ergebnisse wie der Vergleich der Gesamtbelastung in Abbildung 4.10. Das Szenario „Rentenalter erhöhen“ liefert kurz- bis mittelfristig den höchsten Pro-Kopf-Konsum. „Kapitaldeckung einführen“ ist anfänglich das ungünstigste Szenario, holt aber ab dem Jahr 2030 auf und eröffnet nach dem Jahr 2046 die höchsten Konsummöglichkeiten. Das Szenario „Ersatzquote senken“ liegt in dem abgebildeten Zeitraum immer unter dem Szenario „Rentenalter erhöhen“, doch nach dem Jahr 2050 übertrifft der Pro-Kopf-Konsum bei einer Ersatzquote von $30 \%$ den Pro-Kopf-Konsum bei einem höheren Rentenalter. Diese Entwicklung wird durch den nach dem Jahr 2040 steigenden Pro-Kopf-Konsum im Szenario „Ersatzquote senken“ angedeutet, denn im Szenario „Rentenalter erhöhen“ sinkt der Pro-Kopf-Konsum weiter. Allerdings erreicht das Szenario „Ersatzquote senken“ nicht die Konsummöglichkeiten des Szenarios „Kapitaldeckung einführen“. 
Abbildung 4.12: Entwicklung des Pro-Kopf-Konsums in den Politikszenarien (Index $1996=1$ )

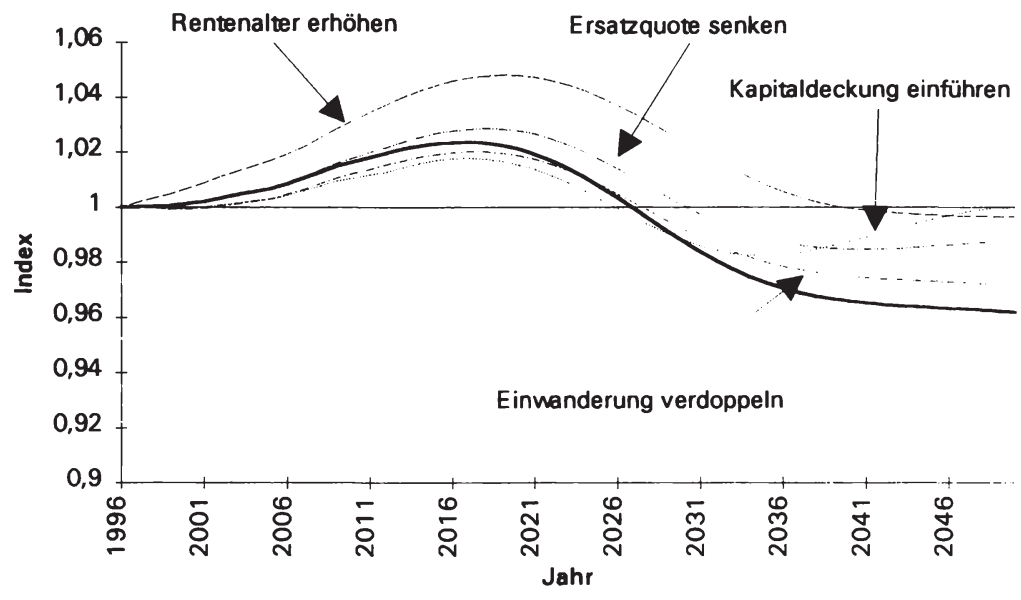

Quelle: Eigene Berechnungen

\section{Zusammenfassende Bewertung}

Obwohl das Szenario „Kapitaldeckung einführen“ den Übergangsgenerationen eine im Vergleich zum Basisszenario deutlich höhere Gesamtbelastung aufbürdet (siehe Abbildung 4.10), ist der Unterschied im Pro-Kopf-Konsum vergleichsweise gering. Offensichtlich kompensieren die bei einer Kapitaldeckung steigenden Accidental Bequests zusammen mit der steigenden Kapitalintensität einen großen Teil der doppelten Bürde der Übergangsgeneration. Der drastische Umstieg auf eine kapitalgedeckte Alterssicherung im Szenario „Kapitaldeckung einführen“ führt also keineswegs zu drastischen Auswirkungen auf den Pro-Kopf-Konsum. Geht man allmählich zu einem kapitalgedeckten Rentensystem über, so wie dies im Szenario „Ersatzquote senken“ geschieht, dann ergeben sich überhaupt keine negativen Konsequenzen für den Pro-Kopf-Konsum. Kombiniert man „Rentenalter erhöhen“ mit „Ersatzquote senken“, dann ist der demographische 
Wandel nicht länger ein Problem, sondern eine Chance: ${ }^{4}$ Sowohl der Pro-KopfKonsum als auch die Gesamtbelastung sind deutlich besser als im Basisszenario.

Weil das Szenario „Kapitaldeckung einführen“ langfristig den höchsten ProKopf-Konsum und die niedrigste Gesamtbelastung verspricht, die Übergangsgenerationen aber kurz- bis mittelfristig belastet, wird nach Möglichkeiten für einen pareto-verbessernden Übergang gesucht. Die vorgeschlagenen Übergangsszenarien gehen von einem ähnlichen Grundgedanken aus: Der Staat (oder die Rentenversicherung) finanzieren die Umstellung vom Umlage- auf das Kapitaldeckungsverfahren durch eine langfristig zurückzahlbare Kreditaufnahme. Dadurch wird ein Teil des Gewinns der zukünftigen Generationen, die den Kredit zurückzahlen müssen, auf die Übergangsgeneration transferiert, die auf diese Weise kompensiert wird. Ein Beispiel für eine Reform nach diesem Muster ist Chile, das seit 1981 das Umlageverfahren durch ein Kapitaldeckungsverfahren ersetzt und seitdem einen nachhaltigen Wirtschaftsaufschwung erlebt, siehe Edwards (1996). ${ }^{5}$ Betrachtet man Europa oder die USA, dann rücken die aktuellen Haushaltsprobleme die Möglichkeiten einer zusätzlichen Verschuldung des Staates in weite Ferne, wodurch die vorgeschlagenen Umstiegsszenarien an praktischer Relevanz verlieren.

Auch aus theoretischer Sicht liefert die Literatur kein eindeutiges Ergebnis zur Frage eines pareto-verbessernden Übergangs vom Umlage- auf das Kapitaldeckungsverfahren. Raffelhüschen (1993) kann im speziellen Rahmen seines Simulationsmodells einen pareto-verbessernden Übergang auf eine kapitalgedeckte Alterssicherung konstruieren. Ähnlich positiv sehen Feldstein (1995) sowie Feldstein und Samwick (1996) die Aussichten für eine Abkehr vom Umlageverfahren. Dagegen schließt Breyer (1996), daß es keine Weiterentwicklung des Rentensystems gibt, die einen pareto-verbessernden Übergang gewährleistet. Auch Kotlikoff (1996) ist skeptisch und argumentiert: Wenn es nicht bedeutende Effizienzgewinne aus dem Abbau der das Arbeitsangebot verzerrenden Beitragssätze gibt, existiert kein pareto-verbessernder Übergang auf ein Kapitaldeckungsverfahren.

4 In Anspielung auf Cutler et al. (1990): An Aging Society: Opportunity or Challenge.

5 Allerdings weist Kotlikoff (1996) darauf hin, daß Chile auf Grund besonderer politischer und ökonomischer Rahmenbedingungen kaum als Beispiel für andere Länder dienen kann. 


\subsection{Kleine offene Volkswirtschaft}

Das Basisszenario und die vier Politikszenarien nehmen eine geschlossene Volkswirtschaft an. Gerade ein Land wie Deutschland, das mit seinen Güter- und Kapitalmärkten stark in den Weltmarkt integriert ist, kann aber nur schwer mit einer geschlossenen Volkswirtschaft gleichgesetzt werden. Daher diskutiere ich in diesem Abschnitt zwei Szenarien - das Basisszenario und das Szenario „Ersatzquote senken“ - für eine kleine offene Volkswirtschaft. Die kleine offene und die geschlossene Volkswirtschaft können als zwei Randfälle aufgefaßt werden, zwischen die sich mittlere und große offene Volkswirtschaften einordnen lassen, denn in der kleinen offenen Volkswirtschaft ist der Zinssatz und damit die Kapitalintensität durch den Weltmarkt exogen gegeben - im Gegensatz zur geschlossenen Volkswirtschaft, in der die Kapitalintensität und der Zinssatz rein endogen bestimmt sind. Größere offene Volkswirtschaften sind Mischformen dieser Randfälle, denn der Zinssatz wird zum einen Teil durch den Weltmarkt und zum anderen Teil durch das Inland bestimmt. Wenn ich hier die beiden für sich genommenen wenig realistischen Randfälle abbilde, dann zeige ich einen Korridor für mögliche Entwicklungen auf, in den sich die realistischeren Fälle einordnen lassen. Existierende Arbeiten von Cutler et al. (1990) sowie BörschSupan (1995c, 1995d und 1996) modellieren die wechselseitigen Abhängigkeiten in einem integrierten Weltmarkt, geben dem Haushaltssektor im Vergleich zu Auerbach et al. (1989) - und damit meinem Modell - eine einfachere Struktur.

Die Simulation einer kleinen offenen Volkswirtschaft geht von einem konstanten Welt-Zinssatz aus. Dieser Welt-Zinssatz bestimmt die Kapitalintensität, den Bruttolohn und den inländischen Output, die gleichfalls konstant sind. Weiterhin endogen sind der Beitragssatz zur umlagefinanzierten Rentenversicherung sowie das Kapital- und Arbeitsangebot der Haushalte, das sich nach dem Welt-Zinssatz und dem Nettolohn richtet. Weil im allgemeinen Gleichgewicht Vollbeschäftigung herrscht, beschäftigen die Unternehmen alle Arbeitnehmer, die Arbeit anbieten, so daß sich aus der konstanten Kapitalintensität und dem Arbeitsangebot der Haushalte das in der Produktion nachgefragte Kapital ergibt. Wegen des großen weltweiten Kapitalmarkts, auf dessen Zinssatz die kleine einheimische Wirtschaft keinen entscheidenden Einfluß hat, kann die Differenz zwischen dem Kapitalangebot der Haushalte und der Kapitalnachfrage der Unternehmen zum gegebenen Welt-Zinssatz auf dem Weltmarkt angelegt bzw. aufgenommen werden. Die Differenz zwischen dem Kapitalangebot der Haushalte und der Kapitalnachfrage der Unternehmen nenne ich „Kapitalbesitz im Ausland“. Positive Werte des Kapitalbesitzes im Ausland zeigen an, daß die inländischen Haushalte mehr Kapital anbieten als die inländischen Unternehmen nachfragen, also findet ein Kapitalexport statt. 
Die kleine offene Volkswirtschaft simuliere ich für zwei Szenarien: das Basisszenario, das ich hier „Basisszenario, offen" nenne, und das Szenario „Ersatzquote senken“, das entsprechend „Ersatzquote senken, offen" heißt. Von den vier Politikszenarien wähle ich „Ersatzquote senken“ aus, weil dieses Szenario das Umlageverfahren auf niedrigem Niveau beibehält und gleichzeitig eine höhere private Ersparnis auslöst, deren Effekt bei offenen Kapitalmärkten besonders interessant ist. Den Welt-Zinssatz setzte ich auf 5,9\% - ein höherer Wert, als der durchschnittliche Zinssatz im Basisszenario der geschlossenen Volkswirtschaft (siehe Abbildung 4.2). Da die Bevölkerung in den meisten anderen Industrieländern langsamer zurückgeht als in Deutschland und in den wirtschaftlich an Bedeutung gewinnenden südostasiatischen Schwellenländern noch zunimmt, wird die Kapitalnachfrage auf dem Weltmarkt relativ größer sein als in Deutschland. Gegenüber der geschlossene Volkswirtschaft bedeutet das einen höheren Welt-Zinssatz. Untersuchungen von Börsch-Supan (1995d, 1996) unterstützen diese Überlegung und weisen darauf hin, daß eine positive wirtschaftliche Entwicklung in Indien und China die Möglichkeiten für wechselseitig vorteilhafte Kapitalexporte der „alternden“ westlichen Länder noch zusätzlich verbessern könnte.

Abbildung 4.13: Entwicklung des Kapitalbesitzes im Ausland in Prozent des inländischen Outputs.

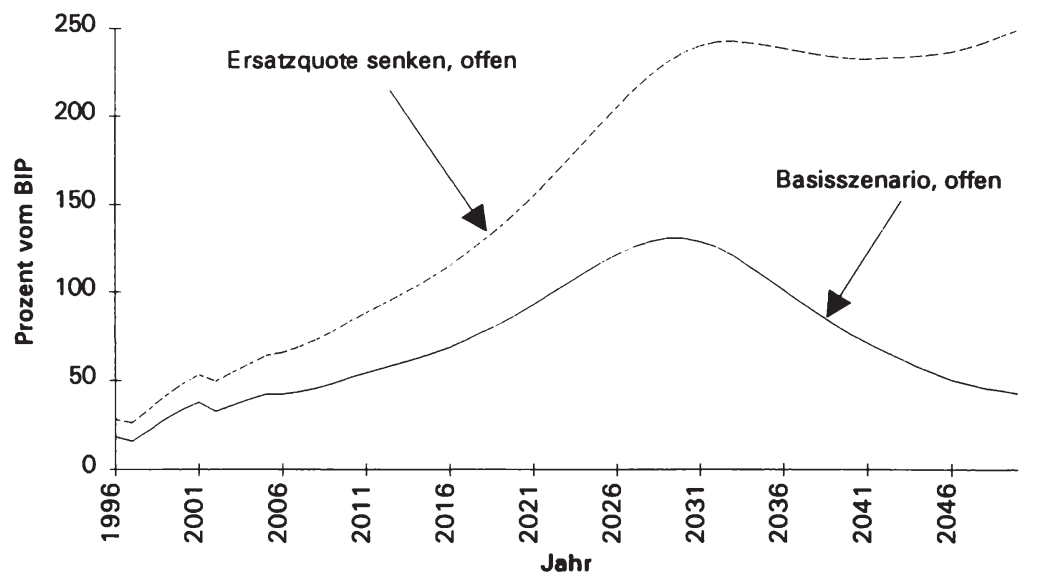

Quelle: Eigene Berechnungen 
Die Entwicklung des Kapitalbesitzes im Ausland steht in einem deutlichen $\mathrm{Zu}$ sammenhang mit der Entwicklung des Zinssatzes (und der Kapitalintensität) in der geschlossenen Volkswirtschaft: Um das Jahr 2030 ist der Zinssatz im Basisszenario und im Szenario „Ersatzquote senken“ in der geschlossenen Volkswirtschaft am niedrigsten. Die Haushalte in der offenen Volkswirtschaft erreichen zur gleichen Zeit den höchsten Kapitalbesitz im Ausland, denn in diesen Jahren ist die Differenz zwischen dem Welt-Zinssatz und dem Zinssatz der geschlossenen Volkswirtschaft maximal. Im „geschlossenen“ Basisszenario nimmt der Zinssatz nach dem Jahr 2030 wieder zu und gleichzeitig geht der Kapitalbesitz im Ausland im „Basisszenario, offen“ deutlich zurück. Anders ist die Situation im Szenario „Ersatzquote senken, offen“, denn durch die im Vergleich zum Basisszenario beträchtlich gestiegene Kapitalbildung zur Altersvorsorge bleiben die Zinsen bei „Ersatzquote senken“ in der geschlossenen Volkswirtschaft dauerhaft auf einem niedrigeren Niveau als im Basisszenario. Also bleibt die Zinsdifferenz zwischen offener und geschlossener Volkswirtschaft hoch und der Kapitalbesitz im Ausland nimmt im Szenario „Ersatzquote senken, offen" auch nach 2030 nur unwesentlich ab. In beiden offenen Szenarien erreicht der Kapitalbesitz im Ausland um das Jahr 2030 beachtliche Größenordnungen. Wahrscheinlich überschätzen die offenen Szenarien die Kapitalbewegungen ins Ausland, denn es ist unwahrscheinlich, daß der Welt-Zinssatz absolut konstant auf hohem Niveau verbleibt. Börsch-Supan (1996) prognostiziert beispielsweise nach dem Jahr 2000 sinkende Welt-Zinssätze, die um das Jahr 2030 ein Minimum erreichen. Im Vergleich zur Autarkie ist dieser Rückgang aber stark gedämpft. Die Idee meiner „offenen“ Szenarien liegt in der grundsätzlichen Einschätzung der Auswirkungen des demographischen Wandels bei freiem Kapitalverkehr - daher verstehe ich Abbildung 4.13 nicht als Prognose der tatsächlichen Höhe der Kapitalexporte.

Die Arbeiten von Cutler et al. (1990) sowie Börsch-Supan (1995d und 1996) haben gezeigt, daß freier Kapitalverkehr helfen kann, die negativen Auswirkungen des demographischen Wandels abzumildern. Abbildung 4.13 legt diesen Schluß ebenfalls nahe, denn aus den Zinseinnahmen des Kapitals im Ausland könnten positive Rückwirkungen auf die inländische Volkswirtschaft entstehen, wodurch die Auswirkungen des demographischen Wandels sogar kompensiert werden könnten. Mit der Betrachtung der Erwerbsstundenquote und des Pro-Kopf-Konsums werde ich diese These näher untersuchen.

Die konstante Kapitalintensität in der kleinen offenen Volkswirtschaft impliziert einen konstanten Bruttolohn. In den beiden offenen Szenarien ist der Welt-Zinssatz höher als in den geschlossenen Szenarien, also sind die Kapitalintensität und der Bruttolohn in der kleinen offenen Volkswirtschaft geringer. Gleichzeitig bedeutet der im Vergleich zu den geschlossenen Szenarien höhere Welt-Zinssatz profitablere Anlagemöglichkeiten. Also bietet sich den Haushalten eine Substitutionsmöglichkeit: Sie können Arbeitseinkommen gegen Zinseinkommen substitu- 
ieren. In den geschlossenen Szenarien besteht diese Substitutionsmöglichkeit praktisch nicht, denn ein höheres Kapitalangebot senkt in einer geschlossenen Volkswirtschaft die Zinsen. Abbildung 4.14 vergleicht die Erwerbsstundenquoten in den offenen Szenarien mit den Erwerbsstundenquoten in den entsprechenden geschlossenen Szenarien.

Abbildung 4.14: Entwicklung der Erwerbsstundenquote in der kleinen offenen Volkswirtschaft

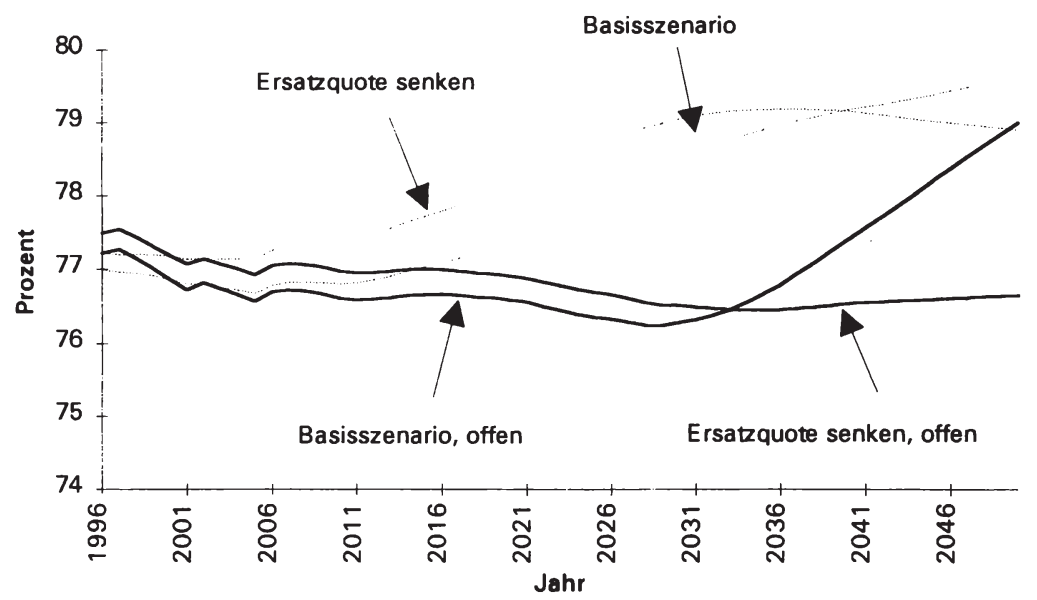

Quelle: Eigene Berechnungen

Nach dem Jahr 2000 sind die Erwerbsstundenquoten in den offenen Szenarien tatsächlich geringer als in den geschlossenen Szenarien, also nehmen die Haushalte die ihnen gebotene Substitutionsmöglichkeit wahr. Wenn der Zinssatz im geschlossenen Basisszenario nach dem Jahr 2030 wieder steigt und sich dem Welt-Zinssatz annähert, dann wird der Abstand zwischen den Erwerbsstundenquoten im offenen und geschlossenen Basisszenario wieder geringer. Dagegen bleibt der Zinsunterschied zwischen dem geschlossenen und offenen Szenario „Ersatzquote senken“ auch nach dem Jahr 2030 bestehen, so daß sich die Erwerbsstundenquoten im offenen und geschlossenen Szenario kaum annähern. Die von den Haushalten der kleinen offenen Volkswirtschaft betriebene Substitution hin zum Zinseinkommen und weg vom Arbeitseinkommen offenbart sich auch im Vergleich der Abbildungen 4.13 und 4.14: Der Kapitalbesitz im Ausland und damit das Zinseinkommen sind invers korreliert mit der Erwerbsstundenquote. 
Der höhere Kapitalbesitz in den offenen Szenarien erhöht auch das Erbschaftsvolumen. Der Anteil der Erbschaften am Lebensarbeitseinkommen für die Kohorten 1969 und 2029 ist in Tabelle 4.2 ausgewiesen.

Tabelle 4.2: Anteil der Erbschaften am Lebensarbeitseinkommen in Prozent

\begin{tabular}{|c|c|c|c|c|}
\hline \multirow[b]{2}{*}{ Kohorte } & \multicolumn{2}{|c|}{ Basisszenario } & \multicolumn{2}{|c|}{ Ersatzquote senken } \\
\hline & geschlossen & offen & geschlossen & offen \\
\hline 1969 & 4,3 & 4,8 & 4,6 & 5,7 \\
\hline 2029 & 6,6 & 7,4 & 8,1 & 14,3 \\
\hline
\end{tabular}

Quelle: Eigene Berechnungen

Das geschlossene Basisszenario weist einen geringeren Anteil der Erbschaften am Netto-Lebensarbeitseinkommen auf als das offene Basisszenario, die Unterschiede sind jedoch geringer als bei den Versionen des Szenarios „Ersatzquote senken“. Besonders für die Kohorte 2029 kommen im Szenario "Ersatzquote senken, offen" zwei Effekt zusammen: Der Betrag der Erbschaften steigt und das Netto-Lebensarbeitseinkommen sinkt. Dieses höhere Gewicht der Erbschaften verstärkt die Substitution von Arbeits- zu Zinseinkommen.

Die Substitution von Arbeitseinkommen zugunsten von Zinseinkommen erhöht die Beitragssätze zur Rentenversicherung in den offenen Szenarien gegenüber den geschlossenen Szenarien. Im Jahr 2030 sind die Beitragssätze in beiden offenen Szenarien um gut einen Prozentpunkt höher als in den geschlossenen Szenarien. Dieses Ergebnis zeigt, daß die Haushalte auf den negativen Anreiz der steigenden Beitragssätze reagieren, wenn sie eine Ausweichmöglichkeit haben. Weil in meinem Simulationsmodell weder die Kapitaleinkünte noch die Erbschaften in die Umlagefinanzierung der Rentenversicherung einbezogen werden, erhöht der freie Kapitalverkehr den Druck auf die Beitragssätze, die ausschließlich das Arbeitseinkommen belasten. Aus diesem Grund schließt Börsch-Supan:

„Will man die Sozialversicherung stabilisieren, muß man also zusätzlich zur Liberalisierung des Handels und Kapitalverkehrs das Steuersystem abändern. Beiträge zur Sozialversicherung müßten von Lohneinkünften auf eine allgemeine Einkommens- oder Konsumsteuer verlagert werden." (Börsch-Supan, 1995d, S.254f) 
Abbildung 4.15: Entwicklung des Beitragssatzes zur umlagefinanzierten Rentenversicherung in der kleinen offenen Volkswirtschaft

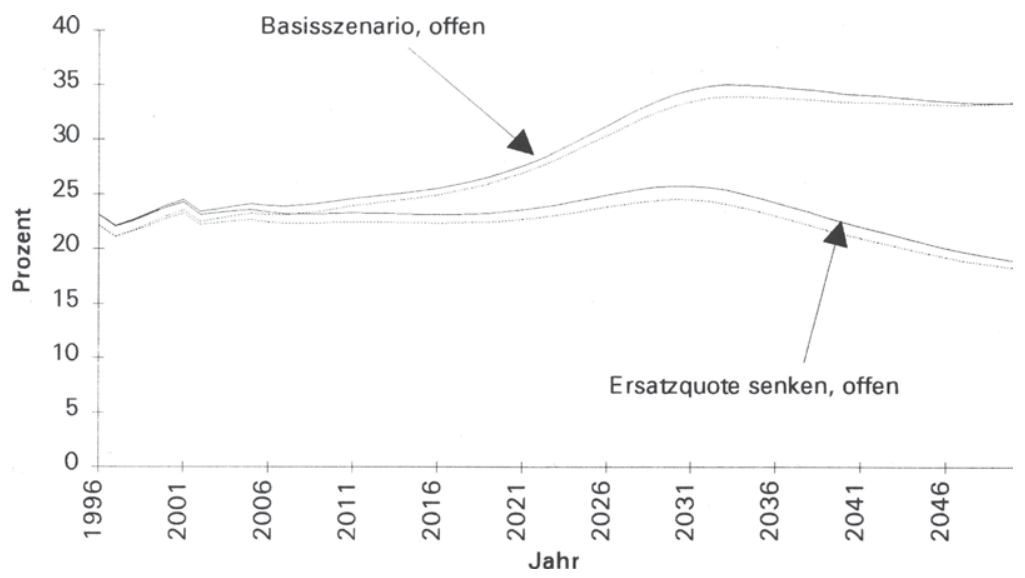

Anmerkung: Die gestrichelten Linien zeigen die Beitragssätze in den jeweiligen Szenarien der geschlossenen Volkswirtschaft.

Quelle: $\quad$ Eigene Berechnungen

Der Pro-Kopf-Konsum entwickelt sich in der kleinen offenen Volkswirtschaft bedeutend besser als in der geschlossenen Volkswirtschaft. Das Basisszenario in der offenen Volkswirtschaft hat in allen Jahren einen höheren Pro-Kopf-Konsum als im Jahr 1996. Im Maximum erreicht der Pro-Kopf-Konsum im Jahr 2019 fast das 1,04-fache des Ausgangsniveaus vom Jahr 1996 und fällt danach bis zum Jahr 2050 wieder auf das Ausgangsniveau von 1996 zurück. Wesentlich günstiger ist die Entwicklung im Szenario „Ersatzquote senken, offen“. Zwar gibt es in den Jahren der größten demographischen Belastung einen minimalen Rückgang, doch ab dem Jahr 2031 steigt der Pro-Kopf-Konsum erneut an. Das Maximum im abgebildeten Zeitraum beträgt das 1,07-fache des Ausgangsniveaus und wird im Jahr 2050 angenommen. 
Abbildung 4.16: Entwicklung des Pro-Kopf-Konsums in der kleinen offenen Volkswirtschaft (Index 1996 = 1)

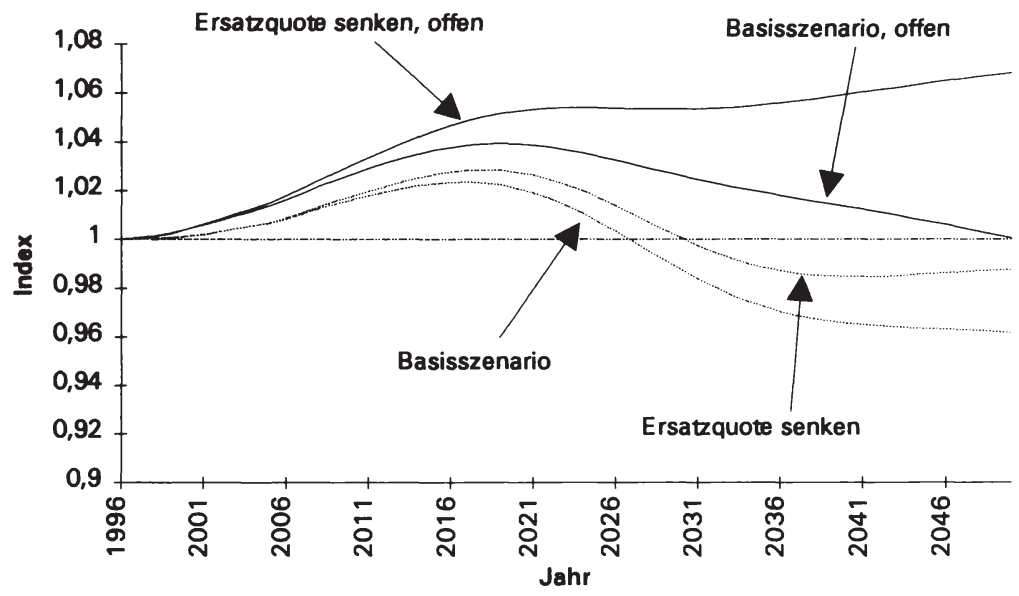

Quelle: Eigene Berechnungen

Alle Szenarien der geschlossenen Volkswirtschaft haben im Jahr 2050 einen geringeren Pro-Kopf-Konsum als im Jahr 1996, wie Abbildung 4.12 zeigt. Aus dem Vergleich der kleinen offenen mit der geschlossenen Volkswirtschaft wird deutlich, welche Vorteile ein ungehinderter Kapitalverkehr für ein Land mit alternder Bevölkerung bringen kann. Genauso wichtig sind die Vorteile für die Partnerländer, denn der höhere Pro-Kopf-Konsum der Inländer wird hauptsächlich im Ausland produziert und ist das Gegengewicht zu den Kapitalexporten der Inländer. Also sind in unterschiedlichen Ländern unterschiedlich verlaufende Bevölkerungsentwicklungen ein weiteres Argument für den freien Welthandel. Das ist das Ergebnis der Arbeiten von Cutler et al. (1990) sowie Börsch-Supan (1995c, 1995d und 1996). Meine Simulation zeigt, daß die positiven Implikationen des freien Welthandels bei einem allmählichen Übergang auf eine teilweise kapitalgedeckte Alterssicherung noch verstärkt werden können. 
Matthias Meier - 978-3-631-75559-4

Downloaded from PubFactory at 01/11/2019 03:34:56AM

via free access 


\section{Zusammenfassung und Ausblick}

\subsection{Zusammenfassung}

Die Frage nach den Auswirkungen des demographischen Wandels auf die gesamtwirtschaftliche Entwicklung ist vor allem für die Weiterentwicklung der Rentenversicherung von großer Bedeutung. Eine partielle Betrachtung der Rentenversicherung greift zu kurz, denn sie kann die von demographischen Veränderungen ausgehenden Rückwirkungen auf die Kapitalbildung und das Arbeitsangebot nur unzureichend berücksichtigen. Daher simuliert diese Arbeit die Auswirkungen des demographischen Wandels in einem allgemeinen Gleichgewichtsmodell, das die Haushalte, den Produktionssektor, die Rentenversicherung und die Bevölkerungsentwicklung verbindet. Ein besonderer Schwerpunkt liegt auf der Modellierung des Sparverhaltens der Haushalte. In früheren Simulationen von allgemeinen Gleichgewichtsmodellen wird das Sparverhalten der Haushalte mit dem klassischen Lebenszyklusmodell abgebildet, das um ein Erbschaftsmotiv bei sicherer Lebenserwartung erweitert ist. Sowohl empirische Überprüfungen dieser einfachen Version des Lebenszyklusmodells, als auch Kalibrierungen früherer Simulationsmodelle zeigen aber, daß das Lebenszyklusmodell das beobachtete Sparverhalten der Haushalte nur unzureichend wiedergibt.

Ein wichtiger Aspekt des demographischen Wandels ist die Veränderung der Lebenserwartung. Modelle mit sicherer Lebenserwartung können diesen Aspekt nicht abbilden. Diesem Mangel versuche ich mit meiner Arbeit entgegenzutreten: Ich integriere ein Lebenszyklusmodell mit Erbschaftsmotiv bei unsicherer Lebenserwartung in die Simulation des allgemeinen Gleichgewichts.

In meiner Simulation des allgemeinen Gleichgewichts vergleiche ich in vier Szenarien die grundsätzlichen Optionen der Politik mit einem Basisszenario, das die gegenwärtigen Regelungen der Rentenversicherung in der Zukunft unverändert beibehält. Die vier Politikszenarien untersuchen, wie sich eine Anhebung des Rentenalters, ein Umstieg auf eine kapitalgedeckte Alterssicherung, eine höhere Zuwanderung und eine auf Grundrentenniveau sinkende Rentenersatzquote auf die wichtigsten endogenen Variablen des Modells auswirken. Zwei weitere Szenarien simulieren eine kleine offene Volkswirtschaft, denn die kleine offene Volkswirtschaft und die geschlossene Volkswirtschaft sind die beiden Randfälle, zwischen die sich die mittleren und großen offenen Volkswirtschaften einordnen lassen. Der Vergleich der Szenarien zeigt, daß verschiedene Reformen der Rentenversicherung ganz unterschiedliche makroökonomische Auswirkungen haben. Wenn die heutigen Regelungen der umlagefinanzierten Rentenversicherung unverändert bestehen bleiben, dann ergeben sich um das Jahr $2030 \mathrm{im}$ Vergleich zu heute nicht nur sehr viel höhere Beitragssätze, sondern auch geringere Konsummöglichkeiten. Damit ist das Basisszenario die schlechteste Politikalternative. 
Aus dieser Feststellung ergibt sich eine klare Implikation für die Wirtschaftspolitik: Es lohnt sich, über Alternativen zu den heutigen Regelungen der Rentenversicherung nachzudenken!

Mit zwei Fragen möchte ich das Nachdenken über Alternativen zu den heutigen Regelungen anregen und die wichtigsten Ergebnisse aus dem Vergleich der Szenarien zusammenfassen:

- Wie kann die Entwicklung der Beitragssätze nachhaltig gedämpft werden?

- Wie kann der Pro-Kopf-Konsum gestärkt werden?

Kombiniert man die beiden Szenarien „Rentenalter erhöhen“ und „Einwanderung verdoppeln“, dann kann man den Beitragssatz auch um das Jahr 2030 auf einem Niveau unter $25 \%$ halten. Insofern besteht die Möglichkeit, die umlagefinanzierte Rentenversicherung langfristig auf einem tragfähigen Niveau zu finanzieren. Eine solche Politik hat aber kaum Auswirkungen auf den Pro-Kopf-Konsum, denn dieser bleibt in etwa auf dem heutigen Niveau. Ursache für dieses ungünstige Ergebnis ist die sinkende Sparquote in den systemerhaltenden Szenarien, deren Rückgang zu einer langfristig wenig veränderten Kapitalintensität führt.

An diesem Punkt setzen die systemverändernden Szenarien an, denn ein teilweiser oder vollständiger Umstieg auf eine kapitalgedeckte Alterssicherung erhöht das gesamtwirtschaftliche Kapitalangebot ganz erheblich. So beträgt die Sparquote im Jahr 2030 im Basisszenario 8,8\%, im Szenario „Ersatzquote senken“ $12,4 \%$ und im Szenario „Kapitaldeckung einführen“ 19,0\%. Dieses höhere Kapitalangebot induziert in den systemverändernden Szenarien eine höhere Kapitalintensität und langfristig auch einen höheren Pro-Kopf-Konsum. Allerdings ist kurz- bis mittelfristig der Pro-Kopf-Konsum geringer und die Summe aus Sparquote und Beitragssatz zur noch existierenden umlagefinanzierten Rentenversicherung höher als in den systemerhaltenden Szenarien. Diese vorübergehend höhere "Gesamtbelastung" ist das Problem, das einen Wechsel zur kapitalgedeckten Alterssicherung aus wirtschaftspolitischer Sicht kurz- bis mittelfristig wenig attraktiv erscheinen läßt.

Bei einem allmählichen Übergang auf eine stärker kapitalgedeckte Rentenversicherung im Szenario „Ersatzquote senken“ ist die Gesamtbelastung gegenüber dem Basisszenario anfänglich geringfügig höher, doch schon ab dem Jahr 2015 kehrt sich der anfängliche Nachteil in einen zunehmend größer werdenden Vorteil um. Da der Pro-Kopf-Konsum im Szenario „Ersatzquote senken“ schon ab dem Jahr 2003 höher ist als im Basisszenario, ist die problematische Übergangsphase spätestens im Jahr 2015 überwunden, denn ab diesem Jahr ist auch die Summe aus Sparquote und Beitragssatz als Proxi für die Gesamtbelastung geringer als im Basisszenario. Daher schließe ich mich der Auffassung von Buslei und Kraus (1996) an: Ein langsam sinkendes Rentenniveaus verursacht nur sehr geringe, 
kurzfristige Wohlfahrtsverluste, hat aber langfristig sehr positive Auswirkungen. In der wirtschaftspolitischen Diskussion sollte eine allmählich auf das Niveau einer Grundrente sinkende Ersatzquote deshalb ernsthaft in Erwägung gezogen werden.

Das Szenario „Ersatzquote senken“ eröffnet einer kleinen offenen Volkswirtschaft noch bessere Möglichkeiten als einer geschlossenen Volkswirtschaft, denn den steigenden privaten Ersparnisse in der kleinen offenen Volkswirtschaft bieten sich auf dem Weltmarkt attraktive Möglichkeiten zur Kapitalanlage, während das zunehmende Kapitalangebot in der geschlossenen Volkswirtschaft die Zinsen senkt. Im Szenario „Ersatzquote senken“ der offenen Volkswirtschaft können die Haushalte durch die höheren Zinseinkünfte einen deutlich höheren Pro-KopfKonsum realisieren als in der geschlossenen Volkswirtschaft.

Dem höhere Zinseinkommen in der offenen Volkswirtschaft steht ein geringeres Arbeitseinkommen gegenüber, denn die Haushalte machen von der ihnen gebotenen Substitutionsmöglichkeit Gebrauch: Sie senken ihr Arbeitsangebot und erhöhen ihr Kapitalangebot. Diese Substitution verstärkt den Druck auf die Beitragssätze zur Rentenversicherung, folglich sind die Beitragssätze in der offenen Volkswirtschaft höher. Ausgelöst wird diese Reaktion durch die ausschließliche Belastung des Arbeitseinkommens mit den Beitragssätzen, man könnte also überlegen, ob ein Teil des auf dem Weltmarkt erzielten höheren Zinseinkommens ebenfalls zur Finanzierung der Renten herangezogen werden sollte. Zusätzlich ist eine Besteuerung der Erbschaften denkbar, denn bei höheren Vermögen steigt das Volumen der Accidental Bequests.

\subsection{Ausblick}

Mit dem Zinseinkommen und den Erbschaften als zusätzlichen Quellen zur Finanzierung der Renten habe ich eine interessante Erweiterungsmöglichkeit für zukünftige Simulationsmodelle gerade angesprochen. Während eine Besteuerung des Zinseinkommens in das Modell von Auerbach und Kotlikoff (1987) schon eingearbeitet wurde, ist die Besteuerung von Erbschaften bei unsicherer Lebenserwartung meines Wissens bisher nicht untersucht worden.

Eine andere Erweiterungsmöglichkeit sehe ich in der „großen offenen Volkswirtschaft", denn gerade Deutschland ist ein Land mit intensiven internationalen Wirtschaftsbeziehungen und einem gewissen Einfluß auf die Weltmarktpreise. Weil sowohl internationale Kapitalmärkte als auch Reaktionen des Arbeitsangebots entscheidenden Einfluß auf die Entwicklungen in den einzelnen Ländern haben, ist eine Verbindung der Simulationen Börsch-Supans (1995c, 1995d und 
1996) mit dem hier entwickelten Modell interessant. Diese Aufgabe ist sowohl aus einem wirtschaftspolitischen als auch aus einem theoretischen Blickwinkel reizvoll. Das wirtschaftspolitische Interesse deckt sich mit dem Thema meiner Arbeit. Die theoretische Herausforderung besteht darin, mit dem hier entwickelten allgemeinen Gleichgewichtsmodell die einzelnen Länder und Regionen in Börsch-Supans Mehr-Länder-Modell zu beschreiben. Dabei muß sorgfältig überlegt werden, wie der Arbeitsmarkt in den einzelnen Ländern und Regionen modelliert wird, denn im Gegensatz zum Kapital ist die Arbeit ortsgebunden.

Schließlich ist die in meiner Untersuchung gewählte Endogenisierung des Arbeitsangebots ein Ansatzpunkt für weitere Forschungen. Bisher ist es nicht gelungen, technischen Fortschritt und endogenes Arbeitsangebot überzeugend miteinander zu vereinbaren. Dieses Problem hängt mit der Separabilitätsannahme zusammen und ist daher theoretisch anspruchsvoll.

Mit einem Schlußwort Breyers als wirtschaftspolitischem Ausblick und Fazit beende ich meine Arbeit:

„Aus den dargestellten Überlegungen sollte deutlich geworden sein, daß zumindest eine Ergänzung des bislang angewendeten Umlageverfahrens durch kapitalfundierte Elemente dazu beitragen kann, das Gesamtsystem der Alterssicherung in den nächsten Jahrzehnten freiheitlicher, effizienter und gerechter zu gestalten." (Breyer, 1996, S. 80)

Dieser Feststellung schließe ich mich uneingeschränkt an. 


\section{Anhang}

\section{A1: Beweis}

$\mathrm{Zu}$ beweisen ist: Wenn Zeitpräferenzrate und Zinssatz gleich sind (d.h. $\rho=r$ gilt), dann stimmen das realisierte Konsumprofil im Sustainable Consumption Modell und im klassischen Lebenszyklusmodell überein.

\section{Notation}

Für den Beweis wird folgende Notation vereinbart:

c Optimaler Konsum im klassischen Lebenszyklusmodell, Lösung der Eulergleichung (2.3).

c Optimaler Konsum im Sustainable Consumption Modell, Lösung der Optimalitätsbedingung (2.16).

$C^{*} \quad$ Sustainable Consumption Variable, entspricht Gleichung (2.14).

$a_{0}$ Exogen gegebenes Anfangsvermögen.

w Exogen gegebenes Arbeitseinkommen.

Zeige per Induktion:

Falls $\rho=r$ gilt, dann ist in allen Perioden $c^{l}=c^{*}=c$.

Induktions-Verankerung:

Wegen $\rho=r$ muß in Periode $1 u^{\prime}\left(c_{1}\right)=u^{\prime}\left(c_{1}{ }^{*}\right)$ gelten und aus der Optimalitätsbedingung (2.16) folgt $c_{1}=c_{1}{ }^{*}$. Dann gilt wegen der Budgetrestriktion (2.13):

$$
c_{1}+\sum_{i=2}^{T}\left(\frac{1}{1+r}\right)^{l-1} c_{1}^{*}=(1+r) a_{0}+\sum_{i=1}^{T}\left(\frac{1}{1+r}\right)^{l-1} w_{1}
$$

Weil $c_{1}=c_{1}{ }^{*}$ gilt, kann diese Gleichung nach $c_{1}$ aufgelöst werden:

$$
\text { (A.1) } \quad c_{1}=c_{1}^{*}=\frac{1}{\sum_{i=1}^{T}\left(\frac{1}{1+r}\right)^{l-1}}\left[(1+r) a_{0}+\sum_{i=1}^{r}\left(\frac{1}{1+r}\right)^{l-1} w_{1}\right]
$$


Nun ist zu zeigen, daß der optimale Konsum in Periode 1 im klassischen Lebenszyklusmodell ebenfalls Gleichung (A.1) genügt. Aus der Eulergleichung (2.3) folgt für $\rho=r$ für alle $t c^{l}=c_{t}$, also wird in allen Perioden gleich viel konsumiert; das Konsumprofil ist flach. Wegen der Budgetrestriktion gilt:

$$
\sum_{i=1}^{T}\left(\frac{1}{1+r}\right)^{l-1} c^{\prime}=(1+r) a_{o}+\sum_{i=1}^{r}\left(\frac{1}{1+r}\right)^{i-1} w_{1}
$$

(A.2) $\quad c^{\prime}=\frac{1}{\sum_{i=1}^{r}\left(\frac{1}{1+r}\right)^{i-1}}\left[(1+r) a_{o}+\sum_{i=1}^{r}\left(\frac{1}{1+r}\right)^{l-1} w_{1}\right]$

Die Gleichungen (A.1) und (A.2) sind gleich, also ist die Induktion verankert.

Induktions-Schluß

Nimm nun an, es gelte $c_{i}=c_{i}^{*}=c^{\prime}$ für die Perioden $i=1, \ldots, t-1$ und zeige: Auch für $t$ gilt $c_{t}=c_{t}{ }^{*}=c^{\prime}$. Ausgangspunkt ist die Budgetrestriktion (2.13):

$$
\begin{aligned}
& \sum_{i=1}^{t-1}\left(\frac{1}{1+r}\right)^{t-1} c^{\prime}+\left(\frac{1}{1+r}\right)^{t-1} c_{t}+\sum_{i=t+1}^{T}\left(\frac{1}{1+r}\right)^{i-1} c_{t}^{*} \\
& =(1+r) a_{o}+\sum_{i=1}^{T}\left(\frac{1}{1+r}\right)^{i-1} w_{1}
\end{aligned}
$$

Wegen der Optimalitätsbedingung (2.16) gilt $u^{\prime}\left(c_{t}\right)=u^{\prime}\left(c_{t}^{*}\right)$, also $c_{t}=c_{t}{ }^{*}$. Es folgt:

$$
\sum_{i=1}^{t-1}\left(\frac{1}{1+r}\right)^{i-1} c^{\prime}+c_{t} \sum_{i=t+1}^{T}\left(\frac{1}{1+r}\right)^{i-1}=(1+r) a_{o}+\sum_{i=1}^{T}\left(\frac{1}{1+r}\right)^{i-1} w_{1}
$$

Diese Gleichung wird nun nach $c_{t}$ aufgelöst und vereinfacht:

$$
c_{t}=\frac{1}{\sum_{i=t}^{T}\left(\frac{1}{1+r}\right)^{l-1}}\left[(1+r) a_{o}+\sum_{i=1}^{r}\left(\frac{1}{1+r}\right)^{l-1} w_{1}-c^{\prime} \sum_{i=1}^{t-1}\left(\frac{1}{1+r}\right)^{l-1}\right]
$$




$$
\begin{aligned}
& c_{t}=\frac{1}{\sum_{i=t}^{T}\left(\frac{1}{1+r}\right)^{i-1}}\left[c^{\prime} \sum_{i=1}^{T}\left(\frac{1}{1+r}\right)^{i-1}-c^{\prime} \sum_{i=1}^{t-1}\left(\frac{1}{1+r}\right)^{i-1}\right] \\
& c_{t}=\frac{1}{\sum_{i=t}^{T}\left(\frac{1}{1+r}\right)^{i-1}} c^{\prime} \sum_{i=t}^{T}\left(\frac{1}{1+r}\right)^{i-1} \\
& c_{t}=c^{\prime}
\end{aligned}
$$

Also ist die Behauptung per Induktion bewiesen ist.

\section{A2: Simulationsprogramm}

Die Grundstruktur des Simulationsprogramms ist in Abbildung 3.5 dargestellt. In diesem Anhang erläutere ich einige Details, die für die numerische Umsetzung des Programms entscheidende Bedeutung haben. Zuerst diskutiere ich die Fixpunktiteration, durch die in einer äußeren Schleife das allgemeine Gleichgewicht hergestellt wird. Um das Kapital- und Arbeitsangebot der Haushalte bestimmen zu können, muß dazu in einer inneren Schleife die Optimierung des Haushaltssektors gelöst werden - eine nichttriviale Aufgabe, die den zweiten Schwerpunkt dieses Anhangs bildet.

\section{Fixpunktiteration}

Die Fixpunktiteration beruht auf einem nichtlinearen SOR-Verfahren (siehe Finck von Finckenstein, 1977). Gesucht wird der Vektor der Kapitalintensität $x=\left\{x_{1}, \ldots, x_{j e n d}\right\}$, der in allen Perioden $1, \ldots, j e n d$ das allgemeine Gleichgewicht auf den Märkten herstellt. Ausgehend von einem beliebigen Startvektor $x^{0}$ lautet die Iterationsvorschrift:

(A.3) $\quad x^{v+1}=\omega x^{v}+(1-\omega) \hat{x}^{v} \quad$ mit $\omega \in(0,1)$

Dabei bezeichnet der Vektor $x^{\nu}$ die Kapitalintensität, die im Produktionssektor gemäß den Gleichungen (3.15) und (3.16) die Vektoren der Zinssätze und Bruttolöhne festsetzt. Zinssätze und Nettolöhne bestimmen ihrerseits im Haushaltssektor das Kapital- und Arbeitsangebot, aus dem der Vektor $\hat{x}^{v}$ berechnet wird, der 
als „Kapitalintensität“ des Haushaltssektor angesehen werden kann. Der SORParameter $\omega$ in der Iterationsvorschrift (A.3) stellt aus den Kapitalintensitäten des Produktions- und Haushaltssektors eine Konvexkombination her, die im nächsten Iterationsschritt $v+1$ im Produktionssektor erneut die Zinssätze und Bruttolöhne bestimmt. In meinem Simulationsprogramm setzte ich für den SOR-Parameter $\omega=0,6$ und gebe damit der Kapitalintensität des Unternehmenssektors ein etwas höheres Gewicht als der Kapitalintensität des Haushaltssektors. Versuche mit anderen Werten für den SOR-Parameter haben gezeigt, daß mit $\omega=0,6$ die schnellste Konvergenz erreicht wird. ${ }^{1}$ Ein höheres Gewicht des Haushaltssektors (beispielsweise $\omega=0,5$ ) führt zu einer erheblich langsameren und "unruhigeren“ Konvergenz: Anfänglich zeigt die Kapitalintensität deutliche Sprünge, die bei einem höheren Gewicht des Unternehmenssektors geglättet werden. Offensichtlich reagiert der Haushaltssektor stärker auf Änderungen der Kapitalintensität als der Unternehmenssektor, der allein durch die beiden Gleichungen (3.15) und (3.16) beschrieben ist und deshalb eine einfachere Struktur als der Haushaltssektor aufweist. Wie sich gleich zeigen wird, ist die Optimierung im Haushaltssektor deutlich komplexer.

Durch die lohnbezogenen Beitragssätze zur umlagefinanzierten Rentenversicherung unterscheiden sich Brutto- und Nettolohn. Das Programmschema in Abbildung 3.5 zeigt, daß die Beitragssätze zusammen mit der Kapitalintensität iteriert werden. Numerisch „sauberer“ wäre es, die Beitragssätze für eine gegebene Kapitalintensität in einer eigenen Schleife zu iterieren und erst dann mit den exakten Beitragssätzen die neue Kapitalintensität zu bestimmen. Eine solche getrennte Iteration der Beitragssätze hätte zur Folge, daß die Optimierung des Haushaltssektors für jeden neuen Beitragssatzvektor neu durchgeführt werden müßte. Da dies der zeitintensivste Teil des Simulationsprogramms ist, habe ich auf eine eigene Iteration der Beitragssätze verzichtet, denn das Konvergenzverhalten der Kapitalintensitäten und der Beitragssätze in der gemeinsamen äußeren Iteration ist problemlos: Beide Vektoren konvergieren gleichzeitig.

\section{Optimierung im Haushaltssektor}

Der kritische Teil des Simulationsprogramms ist die Optimierung im Haushaltssektor. Diese Optimierung muß für jede Kohorte die im Simulationszeitraum lebt durchgeführt werden. Mit dem Existenzbeweis in Abschnitt 2.4.1 gibt es zwar ein Berechnungsschema, für die numerische Umsetzung ist dieses Schema jedoch ungeeignet. Das Problem liegt in den Eigenschaften der Nutzenfunktionen: Der

1 Je nach Szenario benötigt der Algorithmus 10 bis 20 Iterationen um Konvergenz zu erreichen, wobei der Kovergenztest für $\varepsilon=1 \mathrm{e}-5$ als erfullt gilt. 
marginale Nutzen der isoelastischen Konsumnutzenfunktion ist bei zunehmendem Konsum schon schnell kaum noch von null zu unterscheiden. Nimmt der Konsum jedoch $a b$, wird der marginale Konsumnutzen sehr schnell extrem hoch. Diese Eigenschaft von Nutzenfunktionen ist numerisch ungünstig, denn sie führt zu großen Rundungsfehlern. Verwendet man das mit der letzten Periode $T$ beginnende Berechnungsschema aus dem Existenzbeweis, dann muß man so lange über das Endvermögen iterieren, bis das optimale Konsum- und Vermögensprofil zu dem vorgegebenen Anfangsvermögen paßt. Wegen der Eigenschaften der Nutzenfunktionen und den sich daraus ergebenden Rundungsfehlern kann diese Bedingung numerisch nicht exakt erfüllt werden. Das Anfangsvermögen muß aber exakt null sein, denn in meinem Modell entsteht Vermögen ausschließlich durch Sparen oder Erben nach der Haushaltsgründung im 21. Lebensjahr. Nur unter dieser Prämisse ergibt die Aggregation des Haushaltsvermögens das korrekte gesamt-wirtschaftliche Kapitalangebot.

Weil das Anfangsvermögen exakt null sein muß, habe ich das Berechnungsschema aus Abschnitt 2.4.1 von einer Backward Induction in eine Forward Induction umgewandelt:

1. Beginne mit einem beliebigen $c_{21}$ bei vorgegebenem $a_{21}$ als Startwert.

2. Berechne mit $c_{t}, a_{t}$ das Vermögen in der folgenden Periode $a_{t+1}$.

3. Berechne mit $c_{t}, a_{t+1}$ aus der Eulergleichung $c_{t+1}$ :

$$
u^{\prime}\left(c_{t+1}\right)=\frac{1+\rho}{1+r_{t+1}} \frac{1}{1-q_{t+1}} u^{\prime}\left(c_{t}\right)-\frac{1}{1+r_{t+1}} \frac{q_{t+1}}{1-q_{t+1}} v^{\prime}\left(a_{t+1}\right)
$$

4. Solange $t<T-1$ gilt, gehe zurück zu Schritt 2 .

5. Wenn $t=T-1$ ist, dann berechne mit $c_{T-1}, a_{T-1}$ das Endvermögen $a_{T}$.

6. Wenn das Endvermögen $a_{T}$ die Optimalitätsbedingung

$$
0=v^{\prime}\left(a_{T}\right)-(1+\rho) u^{\prime}\left(c_{T-1}\right)
$$

erfüllt, ist die optimale Lösung bestimmt. Wenn nicht, gehe mit einem neuen Startwert $c_{21}$ zurück zu Schritt 1.

Zur Vereinfachung habe ich hier von endogenem Arbeitsangebot abstrahiert, das wegen der Separabilitätsannahme sehr einfach aus dem Konsum berechnet werden kann und keine zusätzlichen Schwierigkeiten verursacht. Die Forward Induction hat den Vorteil, daß sie von dem für die Aggregation korrekten Anfangsvermögen von null ausgeht. Bestehen bleibt das Problem der Rundungsfehler, das 
sich nun auf die Eulergleichung verlagert. Aus zwei Gründen sind die Rundungsfehler dort eher zu tolerieren. Der erste Grund ist theoretisch: Ursache für die numerischen Probleme sind die Nutzenfunktionen, also sollte man die Rundungsfehler auch bei den Nutzenfunktionen belassen. Der zweite Grund ist praktisch: $\mathrm{Da}$ sich die Rundungsfehler mit jeder Funktionsauswertung verstärken, sind die numerisch berechneten Konsum- und Vermögensprofile am Lebensende am stärksten verzerrt. Für die Aggregation ist eine Verzerrung am Lebensende aber weniger problematisch als eine Verzerrung am Anfang des Lebens, denn es gibt nur wenige Haushalte mit hohem Alter, aber viele Haushalte in jungen Jahren.

Die Vorteile der Forward Induction gibt es natürlich nicht umsonst. Erkauft werden sie mit Schritt 3: Die Eulergleichung hat nicht für jede Kombination von $c_{t}$ und $a_{t+1}$ eine Lösung, denn bei bestimmten Kombinationen kann die Differenz auf der rechten Seite negativ werden, der marginale Konsumnutzen ist aber immer positiv. Numerisch kann das optimale Konsumprofil nun sehr schwierig zu bestimmen sein: Weil die Eulergleichung (Schritt 3 der Forward Induction) in der letzten Periode $T$ in die Bedingung für das optimale Endvermögen (Schritt 6 der Forward Induction) übergeht und diese Bedingung gerade null sein muß, nähert sich auch die rechte Seite der Eulergleichung in den letzten Perioden vor $T$ dem Wert null. Durch die Rundungsfehler kann es jetzt leicht dazu kommen, daß das in der Theorie existierende optimale Konsumprofil numerisch nicht getroffen wird, weil die Rundungsfehler kurz vor der letzten Periode $T$ zu einer negativen rechten Seite der Eulergleichung führen.

Tatsächlich bestimmt dieses Problem ganz wesentlich die Umsetzung der Forward Induction in einen Algorithmus. Die Grundidee ist einfach: Man faßt die Optimalitätsbedingung in Schritt 6 als Funktion von $c_{21}$ auf und sucht eine Nullstelle dieser Funktion $\mathbf{z}$.

$$
z\left(c_{21}\right)=v^{\prime}\left(a_{T}\left(c_{21}\right)\right)-(1+\rho) u^{\prime}\left(c_{T-1}\left(c_{21}\right)\right)
$$

Da die Funktion $\boldsymbol{z}$ stetig ist, kann man sehr leistungsfähige Verfahren verwenden - vorausgesetzt, man kennt den Bereich, in dem die Nullstelle liegt. Dieses Initial Bracketing ist aber das schwierigste Verfahren meines Programms. Das Problem der Rundungsfehler, die in der Nähe des optimalen Konsumprofils in den letzten Perioden zu einer negativen rechten Seite der Eulergleichung (Schritt 3) führen können, läßt kaum Spielraum für die Initial Bracket, denn wenn man sich der Nullstelle nähert, wird $z$ numerisch sehr instabil.

Diesem Problem habe ich mit einer sehr „gutmütigen“ Bracketing Prozedur Rechnung getragen. Ich verwende ein rein heuristisches Verfahren, das auf Golden Section Search aufbaut und nicht die Ableitungen der Funktion $z$ benötigt. Wenn die Bracketing Prozedur die Initial Bracket erfolgreich bestimmt, berechne 
ich die Nullstelle von $z$ mit einer Newton-Raphson-Routine, die in wesentlichen Teilen Press et al. (1992) entnommen ist. Wenn die Bracketing Prozedur keine Initial Bracket finden kann, dann arbeitet sie sich bis zur Rechengenauigkeit an das Konsumprofil heran, das „ersmalig“ zu einer negativen rechten Seite der Eulergleichung führt. Dieses von der Bracketing Prozedur berechnete letzte noch zulässige Konsumprofil betrachte ich in diesem Fall als optimales Konsumprofil. Tatsächlich ist die Funktion $z$ für dieses letzte noch zulässige Profil verhältnismäßig klein, so daß ich meine Bracketing Prozedur für eine adäquate Lösung des numerischen Problems halte. 
Matthias Meier - 978-3-631-75559-4

Downloaded from PubFactory at 01/11/2019 03:34:56AM

via free access 


\section{Literaturverzeichnis}

Aaron, Henry (1966): "The Social Insurance Paradox", Canadian Journal of Economics and Political Science, Vol. 32, pp. 371-374.

Abel, Andrew B. (1985): "Precautionary Saving and Accidental Bequests", American Economic Review, Vol. 75 No. 2, pp. 777-791.

Ahlbrecht, Martin and Martin Weber (1995): "Hyperbolic Discounting Models in Prescriptive Theory of Intertemporal Choice", Zeitschrift für Wirtschaftsund Sozialwissenschaften, 115. Jahrgang, Heft 4, pp. 535-568.

Ainslie, George and Nick Haslam (1992): "Hyperbolic Discounting", in George Loewenstein and Jon Elster (eds.): Choice over Time, Russel Sage Foundation, New York.

Alessie, Rob; Annamaria Lusardi and Arie Kapteyn (1995): "Saving and Wealth Holdings of the Elderly, mimeo.

Arrow, Kenneth J. (1974): Essays in the Theory of Risk Bearing, North Holland.

Auerbach, Alan J. and Laurence J. Kotlikoff (1987): Dynamic Fiscal Policy, Cambridge University Press, Cambridge.

Auerbach, Alan J.; Laurence J. Kotlikoff; Robert P. Hagemann and Guiseppe Nicoletti (1989): “The Economic Dynamics of an Ageing Population: The Case of Four OECD Countries", OECD Economic Studies, No. 12, pp. 97130

Barro, Robert J. and Xavier Sala-i-Martin (1995): Economic Growth, McGrawHill, New York.

Bernheim, Douglas B.; Andrei Shleifer and Lawrence H. Summers (1985): "The Strategic Bequest Motive Motive", Journal of Political Economy, Vol 93, No. 6, pp. 1045-1076.

Blanchard, Olivier Jean and N. Gregory Mankiw (1988): "Consumption: Beyond Certainty Equivalence", American Economic Review, Vol. 78, N0. 2, pp.173-177.

Blundell, Richard; Martin Browning and Costas Meghir (1994): "Consumer Demand and the Life-Cycle Allocation of Household Expenditures", Review of Economic Studies, Vol. 61, pp. 57-80. 
Börsch-Supan, Axel (1994): "Savings in Germany - Part 2: Behavior", in James M. Poterba (ed.): International Comparisons of Household Saving, University of Chicago Press, Chicago

Börsch-Supan, Axel (1995a): „Demographie, Arbeitsangebot und die Systeme der sozialen Sicherung“, in Horst Siebert (Hrsg.): Sozialpolitik auf dem Prüfstand, Mohr, Tübingen.

Börsch-Supan, Axel (1995b): "Age and Cohort Effects in Saving and the German Retirement System", Richerche Economiche, Vol. 49, pp. 207-233.

Börsch-Supan, Axel (1995c): "The Consequences of Population Aging for Growth and Savings", Discussion Paper 514-95, Institut für Volkswirtschaftslehre und Statistik, Universität Mannheim.

Börsch-Supan, Axel (1995d): „Die Rolle von Direktinvestitionen bei der regionalen Entwicklung eines alternden Europas“, in B. Gahlen, H. Hesse und H. J. Ramser (Hrsg.): Standort und Region - Neue Ansätze zur Regionalökonomik, Mohr, Tübingen.

Börsch-Supan, Axel (1996): "The Impact of Population Ageing on Savings, Investment and Growth in the OECD Area", in Future Global Capital Shortages - Real Threat or Pure Fiction?, OECD, Paris.

Börsch-Supan, Axel and Konrad Stahl (1991): "Life Cycle Savings and Consumption Constraints", Journal of Population Economics, Vol. 4, pp. 233255.

Boskin, Michael J. (1978): “Taxation, Saving, and the Rate of Interest”, Journal of Political Economy, Vol. 86, No. 2, pp. S3-S27.

Bosworth, Barry; Gary Burtless and John Sabelhaus (1991): "The Decline in Saving: Evidence from Household Survey", Brookings Papers on Economic Activity, Vol. 1,1991, pp. 183-256.

Braun, Hans-Ulrich (1985): „Grundvermögen privater Haushalte Ende 1983 - Ergebnis der Einkommens- und Verbrauchsstichprobe“, Wirtschaft und Statistik, 12/1985, S. 967-974.

Brent, R. P. (1973): Algorithms for Minimization without Derivatives, PrenticeHall, Englewood Cliffs, NJ.

Breyer, Friedrich (1996): „Leitlinien für eine Systemkorrektur in der Rentenversicherung", in Horst Siebert (Hrsg.): Sozialpolitik auf dem Prüfstand, Mohr, Tübingen. 
Browning, Martin (1991): "A Simple Non-Additive Preference Structure for Models of Household Behavior Over Time", Journal of Political Economy, Vol. 99, No. 3, pp. 607-637.

Browning, Martin and Annamaria Lusardi (1995): "Household Saving: Micro Theories and Micro Facts", VSB-CentER Savings Project, Progress Report 29.

Bundesministerium für Arbeit und Sozialordnung (1996a): Euro Atlas - Soziale Sicherheit im Vergleich, Bonn.

Bundesministerium für Arbeit und Sozialordnung (1996b): Programm für mehr Wachstum und Beschäftigung - Maßnahmen im sozialpolitischen Bereich, Bonn.

Buslei, Hermann und Florian Kraus (1996): „Wohlfahrtseffekte eines graduellen Übergangs auf ein niedrigeres Rentenniveau", in Viktor Steiner und Klaus F. Zimmermann (Hrsg.): Soziale Sicherung und Arbeitsmarkt: Empirische Analyse und Reformansätze, ZEW-Wirtschaftsanalysen, Band 6, Nomos, Baden-Baden.

Caballero, Ricardo J. (1991): "Earnings Uncertainty and Aggregate Wealth Accumulation", American Economic Review, Vol. 81, No. 4, pp. 859-871.

Carroll, Christopher D. (1994): "How Does Future Income Affect Current Consumption?", Quarterly Journal of Economics, Vol. 109, pp. 111-147.

Cutler, David M.; James M. Poterba; Louise M. Sheiner and Lawrence H. Summers (1990): “An Aging Society: Opportunity or Challenge?", Brookings Papers on Economic Activity, Vol. 1:1990, pp.1-73.

Danziger, Sheldon; J. van der Gaag; E. Smolensky and M. Taussig (1982): "The Life Cycle Hypothesis and the Consumption Behavior of the Elderly", Journal of Post Keynesian Economics, Vol. 5, pp. 208-227.

Davies, James B. (1981): "Uncertain Lifetime, Consumption, and Dissaving in Retirement", Journal of Political Economy, Vol. 89, No. 3, pp. 561-577.

Deaton, Angus (1992): Understanding Consumption, Oxford University Press, Oxford.

Deaton, Angus and John Muellbauer (1980): Economics and Consumer Behavior, Cambridge University Press, New York.

Deutsche Bundesbank (1993): „Zur Vermögenssituation der privaten Haushalte in Deutschland“, Monatsbericht Oktober 1993, S. 19-32. 
Deutsche Bundesbank (1996): „Die längerfristige Entwicklung des Privaten Verbrauchs in Deutschland und seine Bestimmungsgründe", Monatsbericht Juli 1996, S. 17-29.

Dixit, Avinash K. (1990): Optimization in Economic Theory, 2nd. ed., Oxford University Press, Oxford.

Dustmann, Christian and Matthias Meier (1995): "The Demand for Secondary Education and Parental Characteristics - An Analysis for Germany", paper presented at the 9th Annual Meeting of the European Socienty for Population Economics, Lisbon.

Edwards, Sebastian (1996): "The Chilean Pension Reform: A Pioneering Program", paper presented at the NBER Privatizing Social Security Conference, mimeo.

Feldstein, Martin (1995): "Would Privatizing Sozial Security Raise Economic Welfare?”, NBER Working Paper, No. 5281.

Feldstein, Martin and Andrew Samwick (1996): "The Transition Path in Privatizig Social Security", paper presented at the NBER Privatizing Social Security Conference, mimeo.

Finck von Finckenstein, Karl Graf (1977): Einführung in die Numerische Mathematik, Band 1, Hanser, München.

Flavin, Marjorie (1981): "The Adjustment of Consumption to Changing Expectations About Future Income", Journal of Political Economy, Vol. 89, pp. 974-1009.

Friedman, Milton (1957): A Theory of the Consumption Function, Princeton University Press, Princeton, N.J..

Gale, William G. and John Karl Scholz (1994): "Intergenerational Transfers and the Accumulation of Wealth", Journal of Economic Perspectives, Vol 8. No. 4, pp. 145-160.

Hall, Robert E. (1978): "Stochastic Implications of the Life Cycle-Permanent Income Hypothesis: Theory and Evidence", Journal of Political Economy, Vol. 86, pp. 971-987.

Hall, Robert E. and Frederic Mishkin (1982): "The Sensitivity of Consumption to Transitory Income: Estimates from Panel Data on Households", Econometrica, Vol. 50, pp. 461-481.

Hansen, Lars P. and James J. Heckman (1996): "The Empirical Foundations of Calibration", Journal of Economic Perspectives, Vol. 10, No. 1, pp. 87104. 
Hey, John D. (1983): “Whither Uncertainty?", The Economic Journal, Vol. 93 (conference supplement), pp. 129-138.

Hubbard, R. Glenn (1987): "Uncertain Lifetimes, Pensions and Individual Saving", in Zvi Bodie; John Shoven and David A. Wise (eds.) Issues in Pension Economics, University of Chicago Press, Chicago.

Hubbard, R. Glenn; Jonathan Skinner and Stephen P. Zeldes (1995): "Precautionary Saving and Social Insurance", Journal of Political Economy, Vol. 103, No. 2, pp. 360-399.

Hurd, Michael D. (1987): "Savings of the Elderly and Desired Bequests", American Economic Review, Vol. 77, No. 3, pp. 298-312.

Hurd, Michael D. (1989), "Mortality Risk and Bequests", Econometrica, Vol. 47, No. 4, pp. 779-813.

Hurd, Michael D. (1990): "Research on the Elderly: Economic Status, Retirement, and Consumption and Saving", Journal of Economic Literature, Vol. 28, pp. 565-637.

Jappelli, Tullio and Marco Pagano (1989): "Consumption and Capital Markets Imperfections: An International Comparison", American Economic Review, Vol. 79, pp. 1088-1105.

Kahneman, Daniel and Amos Tversky (1979): "Prospect Theory: An Analysis of Decision Under Risk", Econometrica, Vol. 47, No. 2, pp. 262-291.

Kahneman, Daniel; Jack L. Knetsch and Richard H. Thaler (1991): "The Endowment Effect, Loss Aversion, and Status Quo Bias", Journal of Economic Perspectives, Vol. 5, No. 1, pp. 193-206.

Keynes, John Maynard (1935): The General Theory of Employment, Interest, and Money, reprinted by Harcourt Brace Jovanovich, Orlando, (1964).

Kimball, Miles (1990): "Precautionary Saving in the Small and in the Large", Econometrica, Vol. 58, pp. 53-73.

King, Mervyn (1985): "The Economics of Saving: A Survey of Recent Contributions", in Kenneth J. Arrow and Seppo Honkapohja (eds.): Frontiers of Economics, Basil Blackwell, Oxford.

Kirkpatrick, S.; C. D. Gelatt and M. P. Vecchi (1983): “Optimization by Simulated Annealing", Science, Vol. 220, pp. 671-680.

Kotlikoff, Laurence J. (1988): "Intergenerational Transfers and Savings", Journal of Economic Perspectives, Vol. 2, No. 2, pp. 41-58. 
Kotlikoff, Laurence J. (1996): "Simulating the Privatization of Social Security in General Equilibrium", paper presented at the NBER Privatizing Social Security Conference, mimeo.

Kotlikoff, Laurence J.; John Shoven and Avia Spivak (1986): “The Impact of Annuity Insurance on Savings and Inequality", Journal of Labor Economics, Vol. 4, pp. S183-S207.

Kotlikoff, Laurence J.; John Shoven and Avia Spivak (1987): “Annuity Markets, Saving, and the Capital Stock", in Zvi Bodie; John Shoven and David A. Wise (eds.) Issues in Pension Economics, University of Chicago Press, Chicago.

Kotlikoff, Laurence J. and Lawrence Summers (1981): “The Role of Intergenerational Transfers in Aggregate Capital Accumulation", Journal of Political Economy, Vol. 89, pp. 706-732.

Kydland, Finn E. and Edward C. Prescott (1996): "The Computational Experiment: An Econometric Tool", Journal of Economic Perspectives, Vol. 10, No. 1 , pp. 69-85.

Laibson, David (1995): “Golden Eggs and Hyperbolic Discounting”, mimeo.

Laibson, David (1994): "Mental Accounts, Self-Control, and an Intrapersonal Principal-Agent Problem", mimeo.

Loewenstein, George and Richard H. Thaler (1989): “Anomalies in Intertemporal Choice”, Journal of Economic Perspectives, Vol. 3, No. 4, pp. 181-193.

MaCurdy, Thomas E. (1982): “An Empirical Model of Labour Supply in a LifeCycle Setting", Journal of Political Economy, Vol. 89, No. 6, pp. 10591085.

Miegel, Meinhard (1985): „Grundversorgung und private Alterssicherung - Eine Alternative zum bestehenden Rentenkonzept", in P. Hampe (Hrsg.): Renten 2000, München.

Mirer, Thad (1979): "The Wealth-Age Relation Among the Aged", American Economic Review, Vol. 69, No. 3, pp. 435-443.

Modigliani, Franco (1988): "The Role of Intergenerational Transfers and Life Cycle Saving in the Accumulation of Wealth", Journal of Economic Perspectives, Vol. 2, No. 15-40.

Modigliani, Franco and Richard Brumberg (1954): "Utility Analysis and the Consumption Function: An Interpretation of Cross-Section Data", in Kenneth K. Kurihara (ed.): Post-Keynesian Economics, Rutgers University Press, New Brunswick, N.J.. 
Motel, Andreas und Katharina Spieß (1995): „Finanzielle Unterstützungsleistungen alter Menschen an ihre Kinder - Ergebnisse der Berliner Altersstudie“, Forum Demographie und Politik, Heft 7, SPD-Parteivorstand, Bonn.

Nelder, J.A. and R. Mead (1965): “A Simplex Method for Function Minimization", Computer Journal, Vol. 7, pp. 308-313.

Obstfeld, Maurice (1990): "Intertemporal Dependence, Impatience, and Dynamics", Journal of Monetary Economics, Vol. 26, pp. 45-75.

Owens, Jeffrey (1993): "Taxation and Savings", in Arnold Heertje (ed.): World Savings: An International Survey, Blackwell, Oxford.

Pemberton, James (1993): “Attainable Non-Optimality or Unattainable Optimality: A New Approach to Stochastic Life Cycle Problems", The Economic Journal, Vol. 103, pp. 1-20.

Poterba, James M. (ed.) (1994): International Comparisons of Household Saving, University of Chicago Press, Chicago.

Pratt, John W. (1964): "Risk Aversion in the Small and in the Large", Econometrica, Vol. 32, pp. 122-136.

Press, Wiliam H.; Saul A. Teukolsky; Wiliam T. Vetterling and Brian P. Flannery (1992): Numerical Recipes (2nd ed.), Cambridge University Press, Cambridge.

Raffelhüschen, Bernd (1993): "Funding Social Security Through Pareto-Optimal Conversion Policies", Journal of Economics / Zeitschrift für Nationalökonomie, Suppl. 7, pp. 105-131.

Raffelhüschen, Bernd und Wolfgang Kitterer (1990): „Übergangsprobleme eines Systemwechsels in der sozialen Alterssicherung. Eine dynamische Simulationsanalyse", in Bernhard Felderer (Hrsg.): Bevölkerung und Wirtschaft, Duncker \& Humblot, Berlin.

Rodepeter, Ralf (1995): Prognosen des Arbeitsangebots unter besonderer Beachtung der Altersstrukturverschiebung, unveröffentlichte Diplomarbeit, Lehrstuhl Börsch-Supan, Universität Mannheim.

Schmähl, Winfried (1994a): „Perspektiven der Alterssicherung in Deutschland“, Wirtschaftsdienst, 1994/VIII, S. 390-395.

Schmähl, Winfried (1994b): „Strategien und Maßnahmen künftiger Alterssicherungspolitik in Deutschland“, Wirtschaftsdienst, 1994/X, S. 507-514.

Schmidt, Peter (1995): Die Wahl des Rentenalters, Lang, Frankfurt am Main. 
Schnabel, Reinhold (1994): Das intertemporale Arbeitsangebot verheirateter Frauen, Campus, Frankfurt.

Shefrin, Hersh M. and Richard H. Thaler (1988): "The Behavioral Life-Cycle Hypothesis", Economic Inquiry, Vol. XXVI, pp. 609-643.

Shefrin, Hersh M. and Richard H. Thaler (1992): "Mental Accounting, Saving, and Self-Control", in George Loewenstein and Jan Elster (eds.): Choice over Time, Russell Sage Foundation, New-York.

Sims, Christopher A. (1996): "Macroeconomics and Methodology", Journal of Economic Perspectives, Vol. 10, No. 1, pp. 105-120.

Skinner, Jonathan (1988): "Risky Income, Life-Cycle Consumption and Precautionary Saving", Journal of Monetary Economics, Vol. 22, pp. 237-255.

Sommer, Bettina (1994): „Entwicklung der Bevölkerung bis 2040 - Ergebnis der achten koordinierten Bevölkerungsvorausberechnung“, Wirtschaft und Statistik, 7/1994, S. 497-503.

Statistisches Bundesamt (1975a - 1992a), Fachserie 15: Wirtschafts-rechnungen, Hefte 2 und 4, Wiesbaden.

Statistisches Bundesamt (1972b - 1992b), Statistisches Jahrbuch für die Bundesrepublik Deutschland, verschiedene Jahrgänge, Wiesbaden.

Statistisches Bundesamt (1993): Statistisches Jahrbuch 1993 für das Ausland, Wiesbaden.

Stiglitz, Joseph E., and Andrew Weiss (1981): "Credit Rationing in Markets with Imperfect Information", American Economic Review, Vol. 71, pp. 393410.

Strotz, R.H. (1956): "Myopia and Inconsistency in Dynamic Utility Maximization", Review of Economic Studies, Vol. 23, pp. 165-180.

Summers, Lawrence H. (1981): "Capital Taxation and Accumulation in a Life Cycle Growth Model”, American Economic Review, Vol. 71, pp. 533-544.

Thaler, Richard H. (1990): "Saving, Fungibility, and Mental Accounts", Journal of Economic Perspectives, Vol. 4, No. 1, pp. 193-205.

Thon, Manfred (1991): „Neue Modellrechnungen zur Entwicklung des Erwerbspotentials im bisherigen Bundesgebiet bis 2010 mit Ausblick bis 2030“, Mitteilungen zur Arbeits- und Berufsforschung, Vol. 4/91, S. 673-688.

Tversky, Amos and Daniel Kahneman (1991): "Loss Aversion in Riskless Choice: A Reference-Dependent Model", Quarterly Journal of Economics, Vol. CVI, pp. 1039-1061. 
Verband Deutscher Rentenversicherungsträger (Hrsg.) (1995): Prognos-Gutachten 1995: Perspektiven der gesetzlichen Rentenversicherung für Gesamtdeutschland vor dem Hintergrund veränderter politischer und ökonomischer Rahmenbedingungen, DRV-Schriften, Band 4.

Verband Deutscher Rentenversicherungsträger (1996): Rentenversicherung in Zahlen 1996, Frankfurt am Main.

Wagner, Gert; Andreas Motel; Katharina Spieß und Michael Wagner (1996): "Wirtschaftliche Lage und wirtschaftliches Handeln alter Menschen", in Meyer, K.U und Paul B. Baltes: Die Berliner Altersstudie, Akademie Verlag, Berlin.

Wolf, Elke (1995): Sparprofile älterer Haushalte in der Bundesrepublik Deutschland, unveröffentlichte Diplomarbeit, Lehrstuhl Börsch-Supan, Universität Mannheim.

Yaari, Menahem E. (1965): "Uncertain Lifetime, Life Insurance, and the Theory of the Consumer", Review of Economic Studies, Vol. 32, pp. 137-150.

Zeldes, Stephen (1989): “Consumption and Liquidity Constraints: An Empirical Investigation", Journal of Political Economy, Vol. 97, pp. 305-346. 
Matthias Meier - 978-3-631-75559-4

Downloaded from PubFactory at 01/11/2019 03:34:56AM

via free access 


\section{STAATLICHE ALLOKATIONSPOLITIK IM MARKTWIRTSCHAFTLICHEN SYSTEM}

Band 1 Horst Siebert (Hrsg.): Umweltallokation im Raum. 1982.

Band 2 Horst Siebert (Hrsg.): Global Environmental Resources. The Ozone Problem. 1982.

Band 3 Hans-Joachim Schulz: Steuerwirkungen in einem dynamischen Unternehmensmodell. Ein Beitrag zur Dynamisierung der Steuerüberwälzungsanalyse. 1981.

Band 4 Eberhard Wille (Hrsg.): Beiträge zur gesamtwirtschaftlichen Allokation. Allokationsprobleme im intermediären Bereich zwischen öffentlichem und privatem Wirtschaftssektor. 1983.

Band 5 Heinz König (Hrsg.): Ausbildung und Arbeitsmarkt. 1983.

Band 6 Horst Siebert (Hrsg.): Reaktionen auf Energiepreissteigerungen. 1982.

Band 7 Eberhard Wille (Hrsg.): Konzeptionelle Probleme öffentlicher Planung. 1983.

Band 8 Ingeborg Kiesewetter-Wrana: Exporterlösinstabilität. Kritische Analyse eines entwicklungspolitischen Problems. 1982.

Band 9 Ferdinand Dudenhöfer: Mehrheitswahl-Entscheidungen über Umweltnutzungen. Eine Untersuchung von Gleichgewichtszuständen in einem mikroökonomischen Markt- und Abstimmungsmodell. 1983.

Band 10 Horst Siebert (Hrsg.): Intertemporale Allokation. 1984.

Band 11 Helmut Meder: Die intertemporale Allokation erschöpfbarer Naturressourcen bei fehlenden Zukunftsmärkten und institutionalisierten Marktsubstituten. 1984.

Band 12 Ulrich Ring: Öffentliche Planungsziele und staatliche Budgets. Zur Ertüllung öffentlicher Aufgaben durch nicht-staatliche Entscheidungseinheiten. 1985.

Band 13 Ehrentraud Graw: Informationseffizienz von Terminkontraktmärkten für Währungen. Eine empirische Untersuchung. 1984.

Band 14 Rüdiger Pethig (Ed.): Public Goods and Public Allocation Policy. 1985.

Band 15 Eberhard Wille (Hrsg.): Öffentliche Planung auf Landesebene. Eine Analyse von Planungskonzepten in Deutschland, Österreich und der Schweiz. 1986.

Band 16 Helga Gebauer: Regionale Umweltnutzungen in der Zeit. Eine intertemporale Zwei-Regionen-Analyse. 1985.

Band 17 Christine Pfitzer: Integrierte Entwicklungsplanung als Allokationsinstrument auf Landesebene. Eine Analyse der öffentlichen Planung der Länder Hessen, Bayern und Niedersachsen. 1985.

Band 18 Heinz König (Hrsg.): Kontrolltheoretische Ansätze in makroökonometrischen Modellen. 1985.

Band 19 Theo Kempf: Theorie und Empirie betrieblicher Ausbildungsplatzangebote. 1985.

Band 20 Eberhard Wille (Hrsg.): Konkrete Probleme öffentlicher Planung. Grundlegende Aspekte der Zielbildung, Effizienz und Kontrolle. 1986.

Band 21 Eberhard Wille (Hrsg.): Informations- und Planungsprobleme in öffentlichen Aufgabenbereichen. Aspekte der Zielbildung und Outputmessung unter besonderer Berücksichtigung des Gesundheitswesens. 1986.

Band 22 Bernd Gutting: Der Einfluß der Besteuerung auf die Entwicklung der Wohnungs- und Baulandmärkte. Eine intertemporale Analyse der bundesdeutschen Steuergesetze. 1986.

Band 23 Heiner Kuhl: Umweltressourcen als Gegenstand internationaler Verhandlungen. Eine theoretische Transaktionskostenanalyse. 1987. 
Band 24 Hubert Hornbach: Besteuerung, Inflation und Kapitalallokation. Intersektorale und internationale Aspekte. 1987.

Band 25 Peter Müller: Intertemporale Wirkungen der Staatsverschuldung. 1987.

Band 26 Stefan Kronenberger: Die Investitionen im Rahmen der Staatsausgaben. 1988.

Band 27 Armin-Detlef Rieß: Optimale Auslandsverschuldung bei potentiellen Schuldendienstproblemen. 1988.

Band 28 Volker Ulrich: Preis- und Mengeneffekte im Gesundheitswesen. Eine Ausgabenanalyse von GKV-Behandlungsarten. 1988.

Band 29 Hans-Michael Geiger: Informational Efficiency in Speculative Markets. A Theoretical Investigation. Edited by Ehrentraud Graw. 1989.

Band 30 Karl Sputek: Zielgerichtete Ressourcenallokation. Ein Modellentwurf zur Effektivitätsanalyse praktischer Budgetplanung am Beispiel von Berlin (West). 1989.

\section{ALLOKATION IM MARKTWIRTSCHAFTLICHEN SYSTEM}

Band 31 Wolfgang Krader: Neuere Entwicklungen linearer latenter Kovarianzstrukturmodelle mit quantitativen und qualitativen Indikatorvariablen. Theorie und Anwendung auf ein mikroempirisches Modell des Preis-, Produktions- und Lageranpassungsverhaltens von deutschen und französischen Unternehmen des verarbeitenden Gewerbes. 1991.

Band 32 Manfred Erbsland: Die öffentlichen Personalausgaben. Eine empirische Analyse für die Bundesrepublik Deutschland. 1991.

Band 33 Walter Ried: Information und Nutzen der medizinischen Diagnostik. 1992.

Band 34 Anselm U. Römer: Was ist den Bürgern die Verminderung eines Risikos wert? Eine Anwendung des kontingenten Bewertungsansatzes auf das Giftmüllrisiko. 1993.

Band 35 Eberhard Wille, Angelika Mehnert, Jan Philipp Rohweder: Zum gesellschaftlichen Nutzen pharmazeutischer Innovationen. 1994.

Band 36 Peter Schmidt: Die Wahl des Rentenalters. Theoretische und empirische Analyse des Rentenzugangsverhaltens in West- und Ostdeutschland. 1995.

Band 37 Michael Ohmer: Die Grundlagen der Einkommensteuer. Gerechtigkeit und Effizienz. 1997.

Band 38 Evamaria Wagner: Risikomanagement rohstoffexportierender Entwicklungsländer. 1997.

Band 39 Matthias Meier: Das Sparverhalten der privaten Haushalte und der demographische Wandel: Makroökonomische Auswirkungen. Eine Simulation verschiedener Reformen der Rentenversicherung. 1997. 


\section{Prinzipien wirtschaftlichen Handelns und ihre Anwendung}

\section{Umriß einer Wirtschaftsphilosophie}

Frankfurt/M., Berlin, Bern, New York, Paris, Wien, 1996. 145 S., 1 Abb.

ISBN 3-631-30477-3

br. DM 48.--*

Die enge Verbindung von Wirtschaft und Weltgeschehen verlangt nach einer exakten Untersuchung ihrer Notwendigkeit, Möglichkeiten und Grenzen. Die Ökonomie wird auf ihre erlernbaren theoretischen Prinzipien, die Möglichkeiten ihrer Anwendung und die Bedingungen ihrer praktischen Durchsetzbarkeit hin untersucht. Ergebnis ist eine gründliche Neubestimmung des Verhältnisses von Theorie, Anwendung und Praxis. Die historisch bekannten Wirtschaftsschulen und -richtungen werden auf ihren Geltungsanspruch hinterfragt. Politische und ideologische Rahmenbedingungen werden in ihrem Einfluß auf Privat- und Gemeinwirtschaft erläutert und Modelle zur Überprüfung von Wirtschaftspolitik und Wirtschaftsethik aufgezeigt.

Aus dem Inhalt: Wissenschaftsanspruch, Definition und Methoden der Ökonomie - Einfluß von politischen und ideologischen Rahmenbedingungen . Privat- und Gemeinwirtschaft - Wirtschaftsschulen und Wirtschaftsmodelle . Wirtschaftsrecht und Wirtschaftsethik - Ökonomische Begriffsbildung und Wissenschaft · Ökonomie und Philosophie

Frankfurt/M - Berlin - Bern · New York · Paris · Wien

Auslieferung: Verlag Peter Lang AG

Jupiterstr. 15, CH-3000 Bern 15

Telefon (004131) 9402131

*inklusive Mehrwertsteuer

Preisänderungen vorbehalten 
Matthias Meier - 978-3-631-75559-4

Downloaded from PubFactory at 01/11/2019 03:34:56AM

via free access 